\title{
Abstracts of the XVII Conference of the SEN and the X Meeting of the ACCA
} Spanish Nutrition Society (SEÑ) and the Catalan Association of Food Science (ACCA)

Barcelona, Spain, June, 27-29, 2018

\section{Abstracts}

Guest Editors

Luis A. Moreno Aznar, Zaragoza

Ascensión Marcos Sánchez, Madrid

M. Carmen Vidal Carou, Barcelona 
S. Karger

Medical and Scientific Publishers Basel $\cdot$ Freiburg $\cdot$ Paris $\cdot$ London . New York $\cdot$ Chennai $\cdot$ New Delhi . Bangkok $\cdot$ Beijing $\cdot$ Shanghai $\cdot$ Tokyo Kuala Lumpur $\cdot$ Singapore $\cdot$ Sydney
Disclaimer

The statements, opinions and data contained in this publication are solely those of the individual authors and contributors and not of the publisher and the editor(s). The appearance of advertisements in the journal is not a warranty, endorsement, or approval of the products or services advertised or of their effectiveness, quality or safety. The publisher and the editor(s) disclaim responsibility for any injury to persons or property resulting from any ideas, methods, instructions or products referred to in the content or advertisements.

Drug Dosage

The authors and the publisher have exerted every effort to ensure that drug selection and dosage set forth in this text are in accord with current recommendations and practice at the time of publication. However, in view of ongoing research, changes in government regulations, and the constant flow of information relating to drug therapy and drug reactions, the reader is urged to check the package insert for each drug for any change in indications and dosage and for added warnings and precautions. This is particularly important when the recommended agent is a new and/or infrequently employed drug.
All rights reserved.

No part of this publication may be translated into other languages, reproduced or utilized in any form or by any means, electronic or mechanical, including photocopying, recording microcopying, or by any information storage and retrieval system, without permission in writing from the publisher or system, without permission in writing from the publisher or,
in the case of photocopying, direct payment of a specified fee to the Copyright Clearance Center (see 'General Information')

(C) Copyright 2018 by S. Karger AG

P.O. Box, CH-4009 Basel (Switzerland)

e-ISBN 978-3-318-06373-8

\section{KARGER}




\section{Welcome letter from the Organising Committee}

It is our pleasure to announce the XVII Conference of the Spanish Nutrition Society (SEN) that will be held in Barcelona on June 27-29, 2018. The day before (June 26), in what is now an established tradition in SEÑ, we will be holding the 5th Young Researchers' Meeting, aimed at promoting interaction and knowledge exchange among young people working in the research field of food and nutrition in Spain.

The Spanish Nutrition Society was created in 1978 thanks to the initiative of a group of distinguished researchers, including Drs. Grande Covián, Varela, Mataix, Rojas, Boza, Canosa, Charro, Gálvez, Vázquez, Zorita, Vivanco, De la Morena, Padrón, Fernández Cruz and Tojo. Their fundamental objective was to promote the study of Nutrition in Spain and collaboration with similar societies from other countries, specifically the International Union of Nutritional Sciences (IUNS) and the Federation of European Nutrition Societies (FENS) of which the SEÑ is currently the only Spanish member. Since then, faithful to its founding objectives, SEN has achieved representation in international organizations and has been engaged in promoting the development of Nutrition as a multidisciplinary science, organizing conferences and scientific meetings, sponsoring publications and establishing relationships with other national societies and foundations of related sciences, such as the Spanish Foundation of Nutrition (FEN) and the Ibero-American Nutrition Foundation (FINUT).

The XVII SEÑ Conference of 2018 also integrates the X meeting of the Catalan Association of Food Science (ACCA), a multidisciplinary scientific association created in 1979 by scientists and professionals from different food-related areas, whose objectives include the promotion of various activities in the field of food, nutrition and health, as well as food quality and safety. In 1992, ACCA became an affiliated society of the Science and Technology Section of the Institute of Catalan Studies (IEC) and more recently of the Spanish Federation of Nutrition, Food and Dietetic Societies (FESNAD).

The venue of the Conference will be the emblematic auditorium of the historic School of Medicine and Health Sciences in the Campus Clinic of the University of Barcelona. This is located in the centre of Barcelona, a welcoming and cosmopolitan city on the shores of the Mediterranean, globally recognized for its cultural, financial, commercial and tourist importance, which we are sure you will enjoy.

The meeting dedicated to young researchers on the day before the Conference will be held in Santa Coloma de Gramenet, in the Food and Nutrition Torribera Campus, a thematic campus that seeks to unite the strengths of the University of Barcelona in the field of Food and Nutrition. We invite all our young researchers to actively participate by presenting their works in the form of oral communications.

We encourage you to book these dates in your 2018 calendar and to attend and participate in our Conference, whose programme we have designed with the hope of offering an up-to-date dynamic, with the best possible content and most relevant speakers. Our scientific field will be covered by presentations, round tables, sessions, debates and oral communications, but without neglecting the leisure and social activities that have always been a hallmark of the Spanish Nutrition Society conferences, and for which Barcelona provides an ideal setting. We would also like to extend our invitation to food-related companies and institutions, which will help us to reflect that 5S " (Salubrious, Safe, Sustainable, Social and Satisfactory) food is only achieved with the involvement of everyone.

We hope you will find the proposal of this congress attractive and that we will be able to share enriching experiences. A warm greeting to everyone, with the hope of personally giving you a warm welcome in Barcelona.

M. Carmen Vidal Carou

President of the Organising Committee of the XVII Conference of the Spanish Nutrition Society

Ramón Estruch Riba Montserrat Rivero Urgell

Vice-Presidents of the Organising Committee of the XVII Conference of the Spanish 

The XVII Conference of the Spanish Nutrition Society (SEN) is framed by the slogan " 5 S Food" in reference to five key elements that represent the current complexity and interrelationship of food with other areas of knowledge. That is the reason why a scientific and multidisciplinary tour of all the issues associated with Salubrious, Safe, Sustainable, Social and Satisfactory food will be offered in this occasion.

Salubrious Food (Healthy Food). Connections between lifestyles and chronic non-communicable diseases, and obesity in particular, will be brought up to date. Precision nutrition will also be covered, highlighting the most important advances in nutrigenomics, epigenetics and metabolomic markers. New evidence for the healthy effects of bioactive, prebiotic and probiotic compounds will be presented, without forgetting the topic of food allergies and intolerances, increasingly prevalent in our society.

Safe Food. With a cultural and anthropological overview of the fear and mistrust of food to frame the debate, there will be an analysis of the current status and future challenges of food safety, doubtlessly taking into account the new scenarios of climate change and an aging population. Food authentication will also be focused on, primarily as a tool to analytically validate characteristics related to the origin of food.

Sustainable Food. The connection between the environment and human health is already well established. The World Health Organization (WHO) states textually that, "human health ultimately depends on a society's capacity to manage the interaction between human activities and the physical and biological environments." And they conclude that a quarter of global diseases are due to preventable environmental factors. In this context, the growing awareness of the population of the need to maintain the "health" of the planet in optimum conditions for future generations is also having an influence on food. The circular economy and the new preferences for sustainable and proximity foods pose important communication and regulatory challenges that will also be addressed in this Conference.

Social Food. In this area, in addition to the social importance derived from the reduction of food waste, attention must also be paid to the problem generated by the diffusion of multiple, and often poorly contrasted, nutritional and food content in the media and in the social networks. The analysis of myths and trends on food and diets, as well as the repercussion of current food "infoxication" and possible measures to counteract it, will also focus some debates of this Conference.

Satisfactory Food. All the aforementioned food-related issues can be conditioned by this last one: satisfaction. In post-development societies, the gastronomic value of food is on the rise and a transversal approach to the study of food is incomplete without taking this into account.

The scientific programme of this Conference is included in this special supplement of 'Annals of Nutrition and Metabolism', which contains 9 round tables, 8 extraordinary conferences and 115 abstracts, including 45 oral communications from the $5^{\text {th }}$ Young Researchers' Meeting. All these presentations are dealing with key topics, such as lifestyle and health; nutrigenomics, epigenetics and metabolomics; food bioactive compounds; probiotics and prebiotics; food allergies and intolerances; food safety, sustainable food and gastronomy. We believe that the Conference can facilitate the exchange of information, evidence and experience among professionals who develop their work in any of the "5S" (Salubrious, Safe, Sustainable, Social and Satisfactory) that frames food today.

Finally, we want to express our gratitude to the participating companies for their invaluable help and, very specially, to the members of the organizing and scientific committees, as well as to all speakers, chairpersons and participants who make this Conference possible one more year.

Luis Moreno Aznar

Ascensión Marcos Sánchez

M. Carmen Vidal Carou

Board of the Scientific Committee of the XVII Conference of the Spanish Nutrition Society 



\section{Organizers}

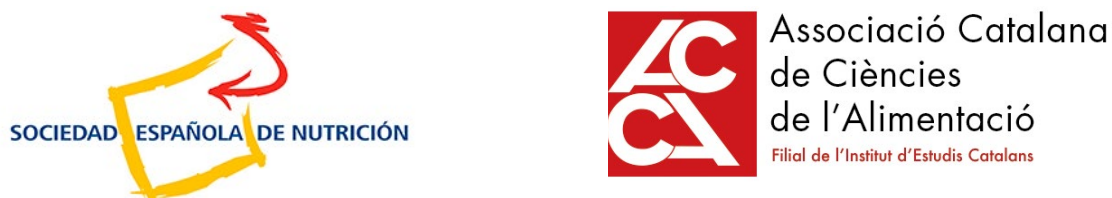

\section{Collaborators}

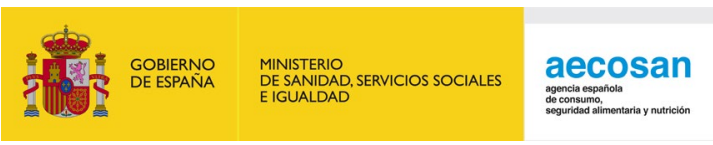

相 Generalitat de Catalunya Departament d'Agricultura, Ramaderia, Pesca i Alimentació

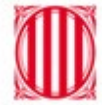

Generalitat de Catalunya Agència de Salut Pública de Catalunya

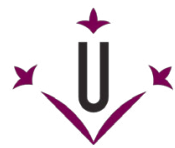

Universitat de Lleida

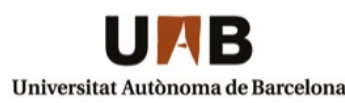

Generalitat de Catalunya

Agència Catalana de Seguretat Alimentària

\section{UNIVERSITAT \\ ROVIRA I VIRGILI}

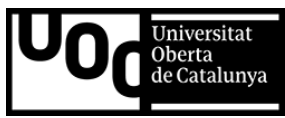





\section{$5^{\text {th }}$ Young Researchers' Meeting}

\section{Tuesday, 26 June 2018}

"Les Voltes" Auditorium, Masia Building

Food and Nutrition Torribera Campus

School of Pharmacy and Food Sciences - Universitat de Barcelona

Santa Coloma de Gramenet

\section{$9.00-9.30$}

\section{Participants Registration}

\section{$9.30-10.00$}

\section{Official Welcome}

\section{Jordi Camarasa}

Dean of the School of Pharmacy and Food Sciences

Josep Boatella

Director of the Food and Nutrition Torribera Campus

Núria Parlon

Mayor of Santa Coloma de Gramenet

Luis Moreno

President of the Spanish Nutrition Society (SEN)

M. Carmen Vidal

President of the Organising Committee of the XVII Conference of the SEN

$10.00-11.00$

\section{Safe, Sustainable and Satisfactory Food \\ Moderators:}

Claudi Mans Teixidó

Universitat de Barcelona. Barcelona

Margarida Castell Escuer

Universitat de Barcelona. Barcelona

\section{JI01}

Shotgun triacylglycerol profiling and chemometrics: towards a fast authentication of olive oil adulteration B. Quintanilla-Casas; S. Vichi; J. Bustamante; M. Simon; F. Guardiola; A. Tres

Universitat de Barcelona. Barcelona

\section{J102}

Presence of anisakids (nematoda) in commonly consumed fish from the Spanish Atlantic and mediterranean coasts and their health risk implication

X. Roca-Geronès; M. Segovia; C. Godínez-González; R. Fisa; I. Montoliu

Universitat de Barcelona. Barcelona

\section{JI03}

Anisakis larvae quantification in commercial fish and fish-derived food using a molecular approach

C. Godínez-González; X. Roca-Geronès; I. Montoliu; R. Fisa Universitat de Barcelona. Barcelona

\section{J104}

A food guide to following a healthy and eco-friendly diet U. Fresán; J. Sabaté Loma Linda University. Loma Linda (USA)

\section{J105}

Cava lees as promoters of the growth of fermentative and probiotic bacteria

S. Hernández-Macias; M.L. Latorre-Moratalla; O. Comas-Basté; S. Sánchez-Pérez; M. Riu-Aumatell; M.T. Veciana-Nogués; E. LópezTamames; M.C. Vidal-Carou

Universitat de Barcelona. Barcelona

\section{J106}

Metabolomics as a tool to study Mediterranean culinary practices: cooking and ingredients affect the metabolic profile of tomato sofrito

J.F. Rinaldi de Alvarenga; P. Quifer-Rada; M. Illan; X. Torrado-Prat; R.M. Lamuela-Raventós

Universitat de Barcelona. Barcelona

\section{J107}

What about legumes as a plant source of the DAO enzyme? O. Comas-Basté; S. Sánchez-Pérez; R.I. Garza-Guajardo; M.L. Latorre-Moratalla; M.T. Veciana-Nogués; M.C. Vidal-Carou Universitat de Barcelona. Barcelona

\section{J108}

Low-histamine diet supplemented with exogenous diamine oxidase enzyme is useful for treating migraine in patients with DAO deficiency

A. Duelo; M. Berbel; H. Mantecon-Laviguerie; O. Comas-Basté; M.L. Latorre-Moratalla; M.T. Veciana-Nogués; M.C. Vidal-Carou Instituto Clínico del Déficit de DAO (ICDDAO). Sant Cugat del Vallès
$11.00-11.30$

\section{Coffee Break}


$11.30-12.45$

\section{Salubrious (healthy) food:}

\section{Lifestyles and Health}

Moderators:

Luis Moreno Aznar

Universidad de Zaragoza. Zaragoza

Maria Izquierdo Pulido

Universitat de Barcelona. Barcelona

\section{J109}

Testing the effect of a theory-based intervention in promoting fruit and vegetable intake among children M. Arrizabalaga; D. Rada; M.P. Portillo; J.R. Mauleón; O. Martínez; I. Etaio; V.M. Rodríguez Universidad del País Vasco - Euskal Herriko Unibertsitatea (UPVEHU). Vitoria-Gasteiz

\section{JI10}

Are vitamin B12 and B9 and total homocysteine concentrations the same in older adults with different levels of physical fitness?

R. Aparicio-Ugarriza; O. López-Torres; G. Palacios; A. Julibert; M.M. Bibiloni; M. González-Gross

Universidad Politécnica de Madrid. Madrid

\section{J11}

Higher adherence to the Mediterranean Diet entails lower cyberbullying behaviour: the mediating role of social competence

N. Ruiz; A. Zaragoza; R. Ferrer; N. Albaladejo, M. Sánchez; V. Clement

Universidad de Alicante. Alicante

\section{J12}

Skin temperature response to a liquid meal intake in adults: the role of gender

L. Ortiz-Alvarez; B. Martinez-Tellez; G. Sanchez-Delgado; H. Xu; F. Acosta; E. Merchan-Ramirez; J. Ruiz

Universidad de Granada. Granada

\section{JI13}

Differences in gut microbiota composition depending on the alcohol consumption pattern in healthy Spanish adults

N. Redondo; A. Gheorghe; L.E. Diaz; B. Villavisencio; A. Marcos; E. Nova

Instituto de Ciencia y Tecnología de los Alimentos y Nutrición (ICTAN-CSIC). Madrid

\section{J14}

Main meal accompaniment influences food and beverage consumption in European adolescents: the Helena study A.M. Santaliestra-Pasias; A. Perez Felez; I. Huybrechts; L. Censi; M.
Gonzalez-Gross; M. Forsner; M. Sjostrom; L.A. Moreno

Universidad de Zaragoza. Zaragoza

\section{J15}

Meal-induced thermogenesis in metabolically healthy but obese adults

H. Xu; G. Sanchez-Delgado; B. Martinez-Tellez; V. Muñoz-Hernández; W.D. Martinez-Avila; F.J. Amaro-Gahete; J. R. Ruiz

Universidad de Granada. Granada

\section{J16}

Relationship between caloric intake and health-related quality of life in the elderly

A. Zaragoza; R. Ferrer; J.A. Hurtado; A. Laguna; M.J. Cabañero; M.F.

Zaragoza; N. Ruiz

Universidad de Alicante. Alicante

\section{J17}

Beverage consumption associated with body composition and the risk of chronic non-communicable diseases in adults

A.L. López-Santana; E.H. Valdés Miramontes; P.J. López-Uriarte

Centro de Investigaciones en Comportamiento Alimentario y Nutrición. México

\section{J18}

Hepatic status and adherence to a Mediterranean dietary pattern in obese subjects with non-alcoholic fatty liver disease

C. Galarregui; I. Abete; I. Cantero; J.I. Monreal; M. Elorz; A. BenitoBoillos; J.A. Martínez; M.A. Zulet

Universidad de Navarra. Pamplona

\section{J19}

Should fresh meat and meat derivatives be placed at different levels of the nutritional pyramid?

S. Sánchez-Pérez; O. Comas-Basté; S. Hernández-Macias; N.C. Muñoz-Esparza; M.L. Latorre-Moratalla; M.T. Veciana-Nogués; M.C. Vidal-Carou

Universitat de Barcelona. Barcelona

\section{J120}

Circadian pattern of skin wrist temperature is associated with anthropometric markers of adiposity in young female adults

M.F. Zeron-Rugerio; A. Díez-Noguera; T. Cambras; M. IzquierdoPulido

Universitat de Barcelona. Barcelona 


\section{$12.45-13.45$}

\section{Workshop:}

Effective oral communication and oratory skills for researchers

\section{Presenter:}

Luis Moreno Aznar.

Universidad de Zaragoza. Zaragoza

Speaker:

\section{M. Ángeles García Asensio.}

Universitat de Barcelona. Barcelona

\section{$13.45-15.00$}

\section{Lunch}

\section{$15.00-15.45$}

\section{Workshop:}

\section{Research and Ethics}

Presenter:

M. Carmen Vidal Carou.

Universitat de Barcelona. Barcelona

Speaker:

Miquel Moretó Pedragosa.

Universitat de Barcelona. Barcelona

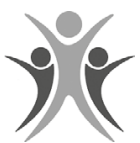

Active break

\section{$15.45-17.15$}

\section{Salubrious (Healthy) Food:}

\section{Lifestyles and health}

\section{Nutrigenomics, epigenetics and metabolomics}

Moderators:

Alfredo Martínez Hernández

Universidad de Navarra. Pamplona

Diego Haro Bautista

Universitat de Barcelona. Barcelona

\section{J121}

Dairy consumption at snack meal occasions and overall diet quality during childhood. IDEFICS / I. Family cohort.

I. Iglesia; T. Intemann; P. De Miguel-Etayo; V. Pala; A. Hebestreit; M. Wolters; G. Eiben; L.A. Moreno

Fundación del Instituto de Investigación Sanitaria Aragón (IIS Aragón). Zaragoza

\section{J122}

Insufficient intake of vitamins in breastfeeding Mexican mothers at two months postpartum.

N.C. Muñoz-Esparza; E.M. Vásquez-Garibay; E. Guzmán-Mercado; A. Larrosa-Haro; E. Romero-Velarde; M.C. Vidal-Carou Universitat de Barcelona. Barcelona

\section{J123}

Vulnerability score in the effectiveness of a children obesity prevention programme: the IDEFICS study. A.M. Ayala-Marín; P. De Miguel-Etayo; J Santabárbara; I. IguacelAzorín; S. De Henauw; L. Lissner; P. Russo; L.A. Moreno Universidad de Zaragoza. Zaragoza

\section{J124}

Recruitment rate in a kindergarten-based, family-involved intervention to prevent childhood obesity across Europe by socio-economic status: The ToyBox study. P. De Miguel-Etayo; C. Summerbell; O. Androutsos; G. Cardon; N. Usheva; P. Socha; Y. Manios; L.A. Moreno Universidad de Zaragoza. Zaragoza

\section{J125}

Dairy consumption and diet quality index in a sample of European children.

E.M. Gonzalez-Gil; A.M. Santaliestra-Pasías; V. Pala; T. Intemann; A. Hebestreit; P. Russo; C. Van Aart; L.A. Moreno Universidad de Zaragoza. Zaragoza

\section{J126}

Effects of alcohol and the non-alcoholic fraction of beer on atherosclerotic biomarkers: a re-analysis based on metabolomics.

P. Quifer; G. Chiva; R. Estruch; R.M. Lamuela-Raventós Universitat de Barcelona. Barcelona

\section{J127}

Effects of Maqui extract (Aristotelia chilensis) on weight control and the metabolic profile of mice fed with a high fat diet.

V. Sandoval; A. Femenias; U. Martinez-Garza; H. Sanz; P.F. Marrero; D. Haro; J. Relat

Universitat de Barcelona. Barcelona

\section{J128}

Food consumption and composition of gut microbiota in young Mexican students.

A. Rodríguez; Z. Reyes; U. López; A. Vázquez; O. Acevedo; J. Rodríguez; C. Gómez; C. Beltrán Universidad de Granada. Granada

\section{J129}

Validation of carnitine palmitoyltransferase 1a as a target of miR-140 in AML12 hepatocytes.

A. Leniz; A. Fernández-Quintela; L. Aguirre; S. Gómez-Zorita; N. Romo; I. Zarrabeitia; M.P. Portillo

Universidad del País Vasco - Euskal Herriko Unibertsitatea (UPVEHU). Vitoria-Gasteiz 
J130

Lipid profile in serum of Alzheimer's disease patients and relationship with MFSD2a fatty acid carrier

M.J. Ruiz; M. Sánzhez-Campillo; A. Gázquez; A.J. Ruiz; E. Larqué; C. Antunez; J. Marín; F. Noguera

Universidad de Murcia. Murcia

\section{J131}

Association of an obesity-predisposing genetic risk score with a set of metabolic and inflammatory traits in a cohort of Spanish children

A. Anguita-Ruiz; A. Rupérez; J. Olza; L. Moreno; A. Gil; G. Bueno; R. Leis; C. Aguilera

University of Granada. Granada

\section{J132}

Differences in the serum kinetic behavior of organic and conventional whole red grape phenolic metabolites

L. Iglesias-Carres; A. Mas-Capdevila; F.I. Bravo; A. Arola-Arnal; B. Muguerza

Universitat Rovira i Virgili. Tarragona

17.15 - 17.45

\section{Coffee Break}

\section{$17.45-19.30$}

\section{Salubrious (healthy) food:}

Food bioactive compounds | Probiotics and prebiotics

Moderators:

\section{Ángel Gil Hernández}

Universidad de Granada. Granada

Ascensión Marcos

Instituto de Ciencia y Tecnología de los Alimentos y Nutrición. Madrid

\section{J133}

Contribution of quercetin metabolites to the triglyceride-lowering effect of the parent compound in 3T3-L1 adipocytes

I. Eseberri; A, Lasa; J. Miranda; A. Mosqueda-Solís; S. GonzálezManzano; C. Santos-Buelga; M.P. Portillo

Universidad del País Vasco - Euskal Herriko Unibertsitatea (UPVEHU). Vitoria-Gasteiz

\section{JI34}

Mediterranean dried fruits are a rich source of phenolic compounds

M. Marhuenda-Muñoz; D. Escobar-Avello; M. Trius; J.F. Rinaldi de Alvarenga; A. Vallverdú-Queralt; R.M. Lamuela-Raventós

Universitat de Barcelona. Barcelona

\section{JI35}

Globin hydrolysates prevent fat deposition in an obesity model

C. Rosell-Cardona; L. Miró; A. Pérez-Bosque; M. Moretó; J. Polo; C. Amat

Universitat de Barcelona. Barcelona

\section{J136}

Role of cocoa theobromine in the metabolism of young Lewis rats

M. Camps-Bossacoma; M. Massot-Cladera; M. Abril-Gil; S. Saldaña-

Ruiz; A. Franch; FJ. Pérez-Cano; M. Castell

Universitat de Barcelona. Barcelona

\section{J137}

Effect of resveratrol in mitochondrial synthesis on the skeletal muscle of rats under standard and energy restriction feeding conditions

I. Milton-Laskibar; L. Aguirre; N. Romo; U. Etxeberria; F.I. Milagro; J.A. Martínez; M.P. Portillo

Universidad del País Vasco - Euskal Herriko Unibertsitatea (UPVEHU). Vitoria-Gasteiz

\section{J138}

Comparison between the polyphenol content in Picual and Hojiblanca extra virgin olive oils from ecological and conventional cropping systems

J. Lozano-Castellón; A. López-Yerena; A. Olmo-Cunillera; P. QuiferRada; A. Vallverdú-Queralt; Pérez Bosch M, B. Jiménez; R.M. Lamuela-Raventós

Universitat de Barcelona. Barcelona

\section{J139}

Relationship between dose and antihypertensive effect for an ace inhibitor chicken feet hydrolysate

A. Mas-Capdevila; L. Iglesias-Carrés; Z. Pons; A. Arola-Arnal; F.I. Bravo; B. Muguerza

Universitat Rovira i Virgili. Tarragona

\section{$\mathrm{J} 140$}

Cherry consumption triggers a differential metabolic response in normoweight and obese Fischer 344 rats exposed to short and long photoperiods

R. Mariné-Casadó; C. Domenech-Coca; J.M. Del Bas; C. Bladé; Ll. Arola; A. Caimari

Eurecat-Centre Tecnològic de Catalunya. Reus

\section{J141}

Influence of the nature of emulsifiers on the bioaccessibility of $\boldsymbol{B}$-carotene encapsulated in nanoemulsions J. Teixé; G. Oms; K. Najman; I. Odriozola Universitat de Lleida. Lleida 
J142

Effect of symbiotic food containing fructo-oligosaccharides from Agave salmiana on the lipid profile of Mexican prepubertal overweight children

O.González-Acevedo; D.P. Portales-Pérez; F. Martínez-Gutiérrez; M. Vega-Cárdenas; D. Vázquez-Maldonado; J.M. Vargas-Morales; C. Godínez-Hernández; A. Rodríguez-Lara; C. Gomez-Llorente Universidad de Granada. Granada

\section{J143}

Lactobacillus fermentum CECT5716 supplementation during rat gestation and lactation periods beneficially impacts their milk and plasma lipid profile I. Azagra-Boronat; A. Tres; M. Massot-Cladera; À. Franch; M. Castell; F. Guardiola; F.J. Pérez-Cano; M.J. Rodríguez-Lagunas Universitat de Barcelona. Barcelona

\section{J144}

GOS/FOS supplementation in suckling rats improves RV diarrhoea by changing the intestinal expression of Toll-like receptors and the microbiota composition M. Massot-Cladera; I. Azagra-Boronat; À. Franch; M.J. RodríguezLagunas; S. Tims; K. Knipping; J. Garssen; F.J. Pérez-Cano Universitat de Barcelona. Barcelona

\section{J145}

Diet-induced gut microbiota dysbiosis can be ameliorated by faecal microbiota transplant

M. Guirro; H. Torrell; A. Costa; P. Herrero; N. Canela; Ll. Arola

Universitat Rovira i Virgili. Tarragona

$19.30-20.00$

\section{Closing of the meeting}





\section{Wednesday 27 June 2018}

$10.00-11.30$

\section{Pre-Congress Session}

The role of nutritionists in educating adults about moderate and responsible drinking.

(Session with simultaneous translation)

\section{Moderator:}

Abel Mariné Font

Universitat de Barcelona.

Speakers:

Carrie Ruxton

UK registered dietitian and health writer

Reference guidelines on moderate drinking guidance. Standard drink equivalence/calorie content and the reasons why this is important for people to understand when choosing a drink and monitoring alcohol intake. Nutrition labelling initiatives for alcohol.

Antonio Villariño Marín.

President of the Spanish Society of Dietetics and Food Sciences.

How health professionals can advise about the responsible consumption of alcohol and provide guidelines for the detection of risky consumption.

$11.00-12.00$

\section{Registration. Accreditation and delivery of docu- mentation}

\section{$12.00-12.30$}

\section{Opening ceremony and official welcome from the authorities}

Albert Cirera Hernández

Vice-rector of entrepreneurship, transfer and innovation at the University of Barcelona.

Francesc Cardellach

Dean of the Faculty of Medicine and Health Sciences.

Jordi Camarasa

Dean of the Faculty of Pharmacy and Food Sciences.

Carmel Mòdol Bresolí

General Director of Food, Quality and Agrifood Industries of the Department of Agriculture, Livestock, Fisheries and Food of Generalitat de Catalunya.
Joan Guix

Secretary of the Public Health Agency of Catalonia (ASPC) of the Department of Health of Generalitat de Catalunya.

Teresa Robledo

Executive Director of AECOSAN - Spanish Agency of Consumption, Food Safety and Nutrition of the Ministry of Health, Social Services and Equality.

Luis Moreno

President of the Spanish Nutrition Society.

M. Carmen Vidal

President of the Organizing Committee.

\section{$12.30-14.00$}

\section{Opening session}

Moderator:

Luis Moreno Aznar

Universidad de Zaragoza. Zaragoza.

Speakers:

\section{Food 5S}

Ana Islas Ramos

Food and Agriculture Organization (FAO).

The importance of salubrious (healthy) food and the NAOS Strategy.

Teresa Robledo de Dios

Agencia Española de Consumo, Seguridad Alimentaria y Nutrición (AECOSAN). Ministerio de Salud, Servicios Sociales e Igualdad. Madrid.

$14.00-15.00$

Working lunch

$15.00-15.30$

\section{Start-Ups Roadshow}

Presenter:

Jose M. Cobo

Insidefood Consulting.

- Diet Creator: Joaquín Torres

- Abouit: Tabaré Majem

- Wefitter: Roger del Sol 


\section{Salubrious (healthy) food}

$15.30-16.00$

\section{Conference}

Congress 5S

Carmen Cabezas i Peña and Gemma Salvador Castell

Health Department. Generalitat de Catalunya. Barcelona

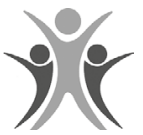

Active break

\section{$16.00-17.30$}

\section{Round Table 1}

Lifestyles and health

Moderators:

Ascensión Marcos Sánchez

Instituto de Ciencia y Tecnología de los Alimentos y Nutrición (ICTAN).

Consejo Superior de Investigaciones Científicas (CSIC). Madrid

Teresa Veciana Nogués

Universitat de Barcelona. Barcelona

Speakers:

Eating habits, breakfast consumption, and obesity in children

Luis Moreno Aznar

Universidad de Zaragoza. Zaragoza

Importance of including physical activity and energy expenditure in nutrition studies

Marcela González Gross

Universidad Politécnica de Madrid. Madrid

Adherence to the Mediterranean diet and mental health: its impact on ADHD in children and adolescents

María Izquierdo Pulido

Universitat de Barcelona. Barcelona

Energy balance and brown fat

Jonatán Ruiz Ruiz

Universidad de Granada. Granada

$17.30-18.00$

Healthy break
$18.00-18.30$

\section{Conferences}

Evidence and controversies in nutrition

Presenter:

Alfredo Martínez Hernández

Universidad de Navarra. Pamplona

Speakers:

Sweeteners and microbiota: are they related?

Ángel Gil Hernández

Universidad de Granada. Granada

Histamine intolerance: from the signs to the evidence M. Carmen Vidal Carou

Universitat de Barcelona. Barcelona

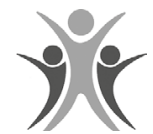

Active break

\section{$18.30-20.00$}

\section{Round Table 2}

Nutrigenomics, epigenetics and metabolomics

Moderators:

Luis Moreno Aznar

Universidad de Zaragoza. Zaragoza

Diego Haro Bautista

Universitat de Barcelona. Barcelona

Speakers:

Nutrigenetics in obesity

Alfredo Martínez Hernández.

Universidad de Navarra. Pamplona

Epigenetic markers in obesity

Concepción Aguilera García.

Universidad de Granada. Granada

Application of metabolomics in health and lifestyle studies

Elvira Larqué Daza

Universidad de Murcia. Murcia

Nutritional metabolomics: dietary assessment through biomarkers

Cristina Andrés Lacueva

Universitat de Barcelona. Barcelona 


\section{Thursday 28 June 2018}

$09.00-10.30$

\section{Round Table 3}

Food Bioactive compounds

Moderators:

Rosaura Farré Rovira

Universidad de Valencia. Valencia

Joana M. Planas Rosselló

Universitat de Barcelona. Barcelona

Speakers:

Diversity of mechanisms of molecular action of bioactive compounds in food

Ángel Gil Hernández

Universidad de Granada. Granada

Resveratrol, pterostilbene and liver steatosis

María del Puy Portillo Baquedano

Universidad del País Vasco UPV/EHU. Bilbao

Effect of culinary treatment on the bioavailability and efficacy of polyphenols

Rosa Lamuela Raventós

Universitat de Barcelona. Barcelona

Nutritional approach to the design of functional foods

Lluis Arola Ferrer

Universitat Rovira i Virgili. Reus

\section{$10.30-11.00$}

Healthy break

$11.00-12.00$

\section{Oral Communications}

Moderators:

Elvira Larqué Daza

Universidad de Murcia. Murcia

Concepción Aguilera García

Universidad de Granada. Granada.

Pedro F. Marrero González

Universitat de Barcelona. Barcelona

6 best communications of the $5^{\text {th }}$ Young Researcher's Meeting

What about legumes as a plant source of the DAO enzyme?

O. Comas-Basté; S. Sánchez-Pérez; R.I. Garza-Guajardo; M.L. Latorre-Moratalla; M.T. Veciana-Nogués; M.C. Vidal-Carou.

Universitat de Barcelona. Barcelona
Association of an obesity-predisposing genetic risk score with a set of metabolic and inflammatory traits in a cohort of Spanish children.

A. Anguita-Ruiz; A. Rupérez; J. Olza; L. Moreno; A. Gil; G. Bueno; R. Leis; C. Aguilera.

University of Granada. Granada

GOS/FOS supplementation in suckling rats improves RV diarrhoea by changing the intestinal expression of Tolllike receptors and the microbiota composition.

M. Massot-Cladera; I. Azagra-Boronat; À. Franch; M.J. Rodríguez-Lagunas; S. Tims; K. Knipping; J. Garssen; F.J. Pérez-Cano.

Universitat de Barcelona. Barcelona

Contribution of quercetin metabolites to the triglyceride-lowering effect of the parent compound in 3T3-L1 adipocytes.

I. Eseberri; A, Lasa; J. Miranda; A. Mosqueda-Solís; S. González-Manzano; C. Santos-Buelga; M.P. Portillo.

Universidad del País Vasco - Euskal Herriko Unibertsitatea (UPV-EHU).

Vitoria-Gasteiz

Dairy consumption and diet quality index in a sample of European children.

E.M. Gonzalez-Gil; A.M. Santaliestra-Pasías; V. Pala; T. Intemann; A. Hebestreit; P. Russo; C. Van Aart; L.A. Moreno.

Universidad de Zaragoza. Zaragoza

Meal-induced thermogenesis in metabolically healthy but obese adults.

H. Xu; G. Sanchez-Delgado; B. Martinez-Tellez; V. Muñoz-Hernández; W.D. Martinez-Avila; F.J. Amaro-Gahete; J. R. Ruiz.

Universidad de Granada. Granada

$12.00-12.30$

Conference

Results of PLENÚFAR 6: Nutrition Education Plan by Pharmacists for people who do physical exercise

Presenter:

Marcela González Gross

Universidad Politécnica de Madrid. Madrid

Speakers:

Aquilino García Perea

Consejo General de Colegios Oficiales de Farmacéuticos. (CGCOF). Madrid

Anna Bach Faig

Colegio Oficial de Farmacéuticos de Barcelona. Universitat Oberta de

Catalunya. Barcelona

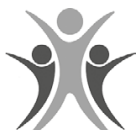

Active break 
$12.30-14.00$

Round Table 4

Probiotics and prebiotics

Moderator:

Guillermo Álvarez Calatayud

President of SEPyP - Spanish Society of Probiotics and Prebiotics. Hospital General Universitario Gregorio Marañón. Madrid

Speakers:

\section{Microbiota and lifestyle}

Ascensión Marcos Sánchez

Instituto de Ciencia y Tecnología de los Alimentos y Nutrición (ICTAN).

Centro Superior de Investigaciones Científicas (CSIC). Madrid

Clinical applications of probiotics and prebiotics

Guillermo Álvarez Calatayud

Hospital General Universitario Gregorio Marañón. Madrid

Comparative trial of a prebiotic versus low-FODMAP diet in patients with irritable bowel syndrome

Fernando Azpiroz Vidaur

Hospital Vall d'Hebron. Barcelona

$14.00-15.00$

Working Lunch

$15.00-15.30$

\section{Start-Ups Roadshow}

Presenter:

Jose M. Cobo

Insidefood Consulting.

- Manzaning: Eva Tomás

- Sharood: Axel Meta

- Urban Chefs: Pep Soler

$15.30-16.00$

\section{Conferences}

\section{Evidence and controversies in nutrition}

Presenter:

María del Puy Portillo Baquedano

Universidad del País Vasco UPV/EHU. Bilbao

Speakers:

Dairy products and cardiometabolic diseases: skimmed or not?

Nancy Babio Sánchez

Universidad Rovira i Virgili. Reus

Moderate consumption of alcohol and health

Ramón Estruch Riba

Hospital Clínic. Universitat de Barcelona. Barcelona
$16.00-17.30$

Round Table 5

Allergies and food intolerances

Moderators:

María Rodríguez Palmero

Catalan Association of Food Science (ACCA). Scientific director of Laboratorios Ordesa. Barcelona

Margarida Castell Escuer

Universitat de Barcelona. Barcelona

Speakers:

Nutritional treatment of cow's milk protein allergy Benjamín Martín Martínez

Associació Catalana de Ciències de l'Alimentació (ACCA). Sociedad Española de Investigación en Nutrición y Alimentación en Pediatría (SEINAP). Barcelona

Cow's milk protein allergy not induced by $\lg E$

Ramón Tormo Carnicé

Hospital Quirón Salud. Barcelona

Intolerance to sugars: from diagnosis to reality

Rosaura Leis Trabazo

Hospital Clínico Universitario de Santiago de Compostela. Santiago de Compostela

Microbiota and probiotics in childhood allergy

Francisco J. Pérez Cano

Universitat de Barcelona. Barcelona

$17.30-18.00$

Healthy break

Social Food

$18.00-18.30$

\section{Conference}

Food fears and mistrust: anthropological perspectives

Presenter:

Miquel Moretó Pedragosa

Universitat de Barcelona. Barcelona

Speaker:

Jesús Contreras Hernández

Universitat de Barcelona. Barcelona

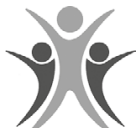

Active break 


\section{Sustainable Food}

\section{$18.30-20.00$}

\section{Round Table 6}

\section{Sustainable food}

Moderators:

Montserrat Rivero Urgell

Associació Catalana de Ciències de l'Alimentació (ACCA). Barcelona

Yvonne Colomer Xena

Fundación Triptolemos. Barcelona

Speakers:

The consequences of agriculture for humanity and the environment

JoanDomènec Ros Aragonés

Institut d'Estudis Catalans (IEC). Barcelona

Determinants of the sustainability of the Mediterranean

Diet

Lluís Serra Majem

Universidad de las Palmas de Gran Canaria

The circular economy in the agri-food sector. The challenge of reducing food waste

Gloria Cugat Pujol

Departament d'Agricultura, Ramaderia, Pesca i Alimentació. Generalitat de Catalunya. Barcelona

Local foods as an element of sustainability

Antonio Montoro Lorite

Universitat de Barcelona. Barcelona

\subsection{0}

\section{Official dinner}

\section{Friday 29 June 2018}

\section{Safe Food}

$09.00-10.30$

\section{Round table 7}

Future challenges for food safety

Moderators:

Carme Chacón Villanueva y Victoria Castell Garralda

Departament de Salut. Generalitat de Catalunya. Barcelona

Catherine Vidal Ortega

Universitat de Barcelona. Barcelona
Speakers:

Effects of climate change on food safety

José Juan Rodríguez Jerez

Universitat Autònoma de Barcelona. Associació Catalana de Ciències de

l'Alimentació (ACCA). Barcelona

\section{Antimicrobial resistances and food}

Marga Arboix Arzo

Universitat Autònoma de Barcelona. Barcelona

Are we moving towards personalized food safety? Sara Bover Cid

Institut de Recerca i Tecnologia Agroalimentàries (IRTA). Barcelona

Verifying food authenticity: the first step towards improving its safety

Stefania Vichi y Alba Tres Oliver

Universitat de Barcelona. Barcelona

$10.30-11.00$

Healthy break

$11.00-11.30$

\section{Internationalization of the SEN}

Presenter:

Ascensión Marcos Sánchez

Instituto de Ciencia y Tecnología de los Alimentos y Nutrición (ICTAN).

Centro Superior de Investigaciones Cientificas (CSIC). Madrid

IUNS Objetives (Union of Nutritional Sciences)

Alfredo Martínez Hernández

Presidente de IUNS. Universidad de Navarra. Pamplona

FINUT Objetives (Fundación Iberoamericana de Nutrición)

Ángel Gil Hernández

Presidente de FINUT. Universidad de Granada. Granada

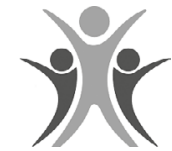

Active break

\section{Satisfactory Food}

\section{$11.30-13.00$}

Round table 8

\section{Contribution of gastronomy to food 5S}

Moderators:

Pere Castells Esqué

Associació Catalana de Ciències de l'Alimentació (ACCA). Gastrocultura

Mediterránea. Barcelona 
Marius Rubiralta Alcañiz

Universitat de Barcelona. Barcelona

Speakers:

Pleasure and health: compatible dimensions Gregorio Varela Moreiras

Universidad CEU San Pablo. Madrid

Gastronomy in hospital diets

Elena Roura Carvajal

Fundació Alicia. Barcelona

Computational gastronomy

Marta Vila Rigat y Axel Bidón-Chanal Badía

Universitat de Barcelona. Barcelona

Gastronomical innovation and sustainability

Victor Quintillà Imbernon

Restaurante Lluerna. Barcelona

$13.00-14.00$

Assembly of SEÑ members

Assembly of ACCA members

(Sessions reserved for SEÑ and ACCA members)

14.00-15.00

Working lunch

$15.00-15.30$

\section{Start-Ups Roadshow}

Presenter:

Jose M. Cobo

Insidefood Consulting

- Nooddle: Carlos Melara

- Co-Cooking: Albert Argilés

- Makeat: J.M Umbert y A. Colominas

\section{Companies for food $5 \mathrm{~S}$}

$15.30-17.00$

\section{Round table 9}

Moderators:

Montserrat Rivero Urgell

Associació Catalana de Ciències de l'Alimentació (ACCA). Barcelona Claudi Mans i Teixidó

Universitat de Barcelona. Barcelona

M. Carmen Vidal Carou

Universitat de Barcelona. Barcelona
Participants:

Asozumos

Contribution of juices to a healthy diet.

María Dolores del Castillo

Head of the Group of Food Biosciences of the Superior Council of Scientific

Research (CSIC)

Cocacola

Reduction of sugar and extension to other categories with new drinks.

Rafael Urrialde

Director of Health and Nutrition of Coca Cola Iberia

Danone

From the product to the sustainable company: the case of Danone as a B Corp company.

Pablo Sánchez.

Country Partner - B Corp Spain

Nestle

Nestlé's proposal for a healthier future.

Laura González

Head of Nutrition, Health and Wellbeing of Nestlé Spain

Unilever

Unilever's Plan for a Sustainable Life.

Ana Palencia

Director of Communication and CSR of Unilever

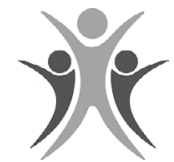

Active break

$17.00-17.30$

Conference

Evidence and controversies in nutrition

Presenter:

Imma Palma Linares

Universitat Ramón LLull. Barcelona

Speakers:

Fruit and vegetables: 5 a day?

Andreu Farrán Codina

Universitat de Barcelona. Barcelona

From the possible to the tangible in weight control diets

Violeta Moizé Arcone

Hospital Clínic de Barcelona. Barcelona 
$17.30-18.00$

40 years of the SEÑ and homage to José Mataix

Presenter:

Luis Moreno Aznar

Universidad de Zaragoza. Zaragoza

Speakers

Salvador Zamora Navarro

Universidad de Murcia. Murcia

Jesús Rodríguez Huertas

Director del Instituto de Nutrición de Granada. Granada

$18.00-18.30$

Healthy break

$18.30-19.30$

Closing Conference

Reflections from experience on the evolution of food sciences (teaching, research and dissemination)

Presenter:

Rosaura Farré Rovira

Universidad de Valencia. Valencia

Speaker:

Abel Mariné Font

Universitat de Barcelona. Barcelona

Homage to Abel Mariné Font

$19.30-20.00$

Conclusions, awards ceremony and closing 



\section{Nutritionals of Metabolism}

\author{
Reviewers: \\ Cristina Andrés Lacueva \\ Universitat de Barcelona \\ José Manuel Ávila Torres \\ Sociedad Española de Nutrición \\ Nancy E. Babio Sánchez \\ Universitat Rovira i Virgili \\ Ramón Estruch Riba \\ Universitat de Barcelona \\ Rosaura Farré Rovira \\ Universidad de Valencia \\ Aquilino García Perea \\ Consejo General de Colegios Oficiales de Farmacéuticos \\ Marcela González-Gross \\ Universidad Politécnica de Madrid \\ Diego Haro Bautista \\ Universitat de Barcelona \\ María Izquierdo Pulido \\ Universitat de Barcelona \\ Ascensión Marcos Sánchez \\ Consejo Superior de Investigaciones Científicas \\ Pedro Marrero Gonzalez \\ Universitat de Barcelona \\ Alfredo Martinez Hernández \\ Universidad de Pamplona \\ Benjamín Martín Martínez \\ Sociedad Española de Investigación en Nutrición y Alimentación \\ en Pediatría
}

Luis A. Moreno Aznar

Universidad de Zaragoza

Montserrat Rivero Urgell

Associació Catalana de Ciències de l'Alimentació

Gregorio Varela Moreiras

Universidad CEU San Pablo

Teresa Veciana Nogués

Universitat de Barcelona

M. Carmen Vidal Carou

Universitat de Barcelona

Josep Vidal Cortada

Hospital Clínic de Barcelona

Salvador Zamora Navarro

Universidad de Murcia

\section{Oral Abstract Presentations}

001

\section{DAIRY CONSUMPTION AT SNACK MEAL OCCA- SIONS AND OVERALL DIET QUALITY DURING CHILDHOOD. IDEFICS/I.FAMILY COHORT}

\author{
${ }^{(1)}$ I. Iglesia; ${ }^{(2)}$ T. Intemann; ${ }^{\left({ }^{3}\right)}$ P. De Miguel-Etayo; ${ }^{(4)} \mathrm{V}$. Pala; ${ }^{\left({ }^{5}\right)}$ A. \\ Hebestreit; ${ }^{(5)}$ M. Wolters; ${ }^{(6)}$ G. Eiben; ${ }^{(3)}$ L.A. Moreno. \\ ${ }^{(1)}$ Growth, Exercise, NUtrition and Development (GENUD) \\ Research Group. Fundación del Instituto de Investigación \\ Sanitaria Aragón (IIS Aragón). Zaragoza. Spain; ${ }^{(2)}$ Institute of \\ Statistics. Bremen University. Bremen. Germany; ${ }^{(3)}$ Growth, \\ Exercise, NUtrition and Development (GENUD) Research \\ Group. Facultad de Ciencias de la Salud. Universidad de \\ Zaragoza. Zaragoza. Spain; ${ }^{(4)}$ Epidemiology and Prevention \\ Unit, Department of Preventive \& Predictive Medicine. \\ Fondazione IRCCS Istituto Nazionale dei Tumori. Milan. Italy; \\ ${ }^{(5)}$ Leibniz Institute for Prevention Research and Epidemiology- \\ BIPS. Bremen. Germany; ${ }^{(6)}$ Department of Public Health and \\ Community Medicine. University of Gothenburg. Gothenburg. \\ Sweden.
}

Introduction: Evidence on whether dairy foods have a negative or positive influence on the overall diet quality in childhood is controversial; moreover, consumption at snack moments has been scarcely investigated.

Objectives: To describe the consumption of dairy products at snack meal occasions during childhood and to investigate its relationship with the overall diet quality (cross-sectionally and longitudinally).

Methods: From the Identification and prevention of Dietaryand lifestyle- induced health EFfects In Children and infantS (IDEFICS) study, data was obtained on the lifestyle and socioeconomic status of 8,807 children aged 2 to 9.9 years from eight European countries at baseline (2007-2008). From those, 5,085 children were re-contacted after two years (2009-2010), and 1,991 were included in the second follow-up and underwent anthropometric and dietary assessment after four years (2013-2014). Dietary intake was assessed by validated 24-hour dietary recalls (HDR). To compute the diet quality index (DQI), food frequency questionnaire (FFQ) and HDR data were used in combination. Associations between dairy consumption and DQI were investigated using multiple regression.

Results: Higher DQI scores were associated with the consumption of milk + yogurt $(\mathrm{p}=0.04)$, and cheese $(\mathrm{p}<0.001)$ at snack meal occasions in T0; with yogurt $(\mathrm{p}<0.001)$, milk + yogurt $(\mathrm{p}<0.001)$, 
and cheese $(\mathrm{p}<0.001)$ in $\mathrm{T} 1$; and only with cheese $(\mathrm{p}=0.05)$ in $\mathrm{T} 3$. In contrast, those consuming sweetened milk or milky desserts in T0 had significantly lower DQI scores $(\mathrm{p}=0.03)$. When considering T0 and T1 and all snack moments, consumers of milk $(\mathrm{p}=0.02)$, yogurt $(\mathrm{p}<0.001)$ or cheese $(\mathrm{p}<0.001)$ had significantly higher DQI scores than non-consumers, as occurred with the consumers of cheese in T1 and T3 ( $\mathrm{p}=0.03)$.

Conclusions: Consumption of milk, yogurt and cheese at snack meal occasions is associated with a higher diet quality and their consumption outside of the main meals may be a good strategy to improve energy balance throughout childhood.

Conflict of interest: Authors declare no conflict of interest. Key words: dairy / snacks / diet quality / children.

\section{2 \\ CIRCADIAN PATTERN OF SKIN WRIST TEMPERA- TURE IS ASSOCIATED WITH ANTHROPOMETRIC MARKERS OF ADIPOSITY IN YOUNG FEMALE ADULTS}

\author{
${ }^{(1)}$ M.F. Zeron-Rugerio; ${ }^{(2)}$ A. Díez-Noguera; ${ }^{(2)}$ T. Cambras; ${ }^{(1)} M$. \\ Izquierdo-Pulido. \\ (1)Department of Nutrition, Food Sciences and Gastronomy. \\ School of Pharmacy and Food Sciences. University of Barcelona. \\ Barcelona. Spain; (2)Department of Biochemistry and Physiology \\ School of Pharmacy and Food Sciences. University of Barcelona. \\ Barcelona. Spain
}

Introduction: The circadian pattern of wrist temperature (WT) can be used as a marker of the circadian system (CS) functioning, and has been associated with health in humans. Body mass index (BMI) and anthropometric markers of adiposity such as fat mass (FM) have been associated with disturbances in the CS (chronodisruption) in overweight and obese adults.

Objectives: To study the association between the circadian pattern of WT, BMI and anthropometric markers of adiposity in young female adults.

Methods: One hundred and ten females (22.4 \pm 2.9 years) were included in a cross-sectional study. WT was measured every for 6 consecutive days (iButton) and body composition with TANITA. Cosinor analysis was used to characterize the WT rhythm, calculating its mesor (mean value of temperature rhythm), amplitude (difference between the maximum or minimum value of temperature and the mesor), and acrophase (timing of the maximum value). The 12-hour rhythm (P2) was also studied. The stability of the rhythm was studied with the Raleigh test (RT) and the percentage of variance (PV) explained by the rhythm.

Results: BMI, FM and waist circumference were positively associated with the acrophase $(\mathrm{p}=0.005)$. On the contrary, negative associations were found between amplitude, BMI $(\mathrm{p}=0.008)$ and FM ( $\mathrm{p}=0.041)$, while RT and PR associated negatively with BMI $(p=0.026)$ as well as PR $(p=0.005) . P 2$ was negatively associated with FM ( $\mathrm{p}=0.034)$.
Conclusions: In a young female adult population, BMI, FM and waist circumference are associated with a delay in the timing of the maximum value of temperature and a less robust rhythm. Moreover, less stable rhythms are found when BMI and FM are higher. Our findings indicate that CS functioning and body composition are associated in healthy subjects, and suggest the role of skin temperature rhythm as a predictor of health status.

Conflicts of interest: Authors declare no conflict of interest.

Key words: circadian rhythm / adiposity / BMI.

\section{3 \\ TESTING THE EFFECT OF A THEORY-BASED IN- TERVENTION IN PROMOTING FRUIT AND VEGE- TABLE INTAKE AMONG CHILDREN}

\author{
${ }^{(1)}$ M. Arrizabalaga; (2)D. Rada; (1)M.P. Portillo; ${ }^{(3)}$ J.R. Mauleón; ${ }^{(1)}$ O. \\ Martínez; ${ }^{(1)}$ I. Etaio; ${ }^{(1)}$ V.M. Rodríguez. \\ (1)Department of Pharmacy and Food Science. Faculty of \\ Pharmacy. University of the Basque Country UPV/EHU. Vitoria- \\ Gasteiz. Spain; ${ }^{(2)}$ Department of Preventive Medicine and Public \\ Health. Faculty of Pharmacy. University of the Basque Country \\ UPV/EHU. Vitoria-Gasteiz. Spain; ${ }^{(3)}$ Department of Sociology 2. \\ Faculty of Pharmacy. University of the Basque Country UPV/EHU. \\ Vitoria-Gasteiz. Spain.
}

Introduction: A controlled intervention was carried out based on the Theory of Planned Behavior (TPB), a behavioral theory that has proved to be one of the most effective at changing infant fruit and vegetable (FV) intake patterns.

Objectives: The main purpose of the study was to evaluate the effectiveness of an intervention based on TPB in increasing FV consumption in schoolchildren aged 8 to 10 .

Methods: In a randomized control trial the intervention group (classrooms $n=4$; children $n=90$ ) received 14 sessions of 60 minutes during an academic year (October-June) but not the control group (classrooms $n=4$; children $n=95$ ). A multidisciplinary team designed the sessions based on TPB and they were directed at modifying the behavioral determinants and the FV intake itself, which were evaluated (before/after) using validated 7-day food records, 24-hour reminders and specifically designed questionnaires.

Results: FV intake increased by 0.45 servings/day in the intervention group ( $\mathrm{p}=0.001 ; \mathrm{CI} 95 \%=0.17-0.74)$. In contrast, the control group did not change its FV consumption after the intervention. Attitude, behavioral control and intention increased in both groups (all, $\mathrm{p}<0.05)$. Subjective norms were maintained in the intervention group and decreased in the control group $(\mathrm{p}=0.04)$. Multiple linear regressions showed an association between a higher FV pre-post change with having received the intervention $(\mathrm{B}=0.339 ; \mathrm{p}=0.030)$ and with lower baseline $\mathrm{FV}$ intake $(\mathrm{B}=-0.436$; $\mathrm{p}=0.000)$ after adjusting the model for gender and family economic level $(\mathrm{R} 2=0.235)$.

Conclusions: The intervention was moderately effective in modifying both the determinants of behavior and FV consump- 
tion. The similar pattern in the evolution of the determinants within both groups may be due to contamination between groups. In addition, it seems that baseline FV intake limits the effect of the intervention. Therefore, the program could be more effective in populations with a lower baseline FV intake.

Conflict of interest: Authors declare no conflict of interest. Key words: intervention / fruit / vegetable / behavior / children.

\section{4 \\ VULNERABILITY SCORE IN THE EFFECTIVENESS OF A CHILDREN OBESITY PREVENTION PRO- GRAMME: THE IDEFICS STUDY.}

\author{
${ }^{(1)}$ A.M. Ayala-Marín; ${ }^{(1,2)}$ P. De Miguel-Etayo; ${ }^{(3,4)}$ J. Santabárbara; \\ ${ }^{(1,2)}$ I. Iguacel-Azorín; ${ }^{(5)}$ S. De Henauw; ${ }^{(6)}$ L. Lissner; ${ }^{(7)}$ P. Russo; ${ }^{(1,2)}$ L.A. \\ Moreno.
}

${ }^{(1)}$ Growth, Exercise, NUtrition and Development (GENUD)

Research Group. Departamento de Fisiatría y Enfermería. Universidad de Zaragoza. Zaragoza. Spain; ${ }^{(2)}$ CIBERobn. Instituto de Salud Carlos III. Madrid. Spain; ${ }^{(3)}$ Departmento de Microbiología, Medicina Preventiva y Salud Pública. Universidad de Zaragoza. Zaragoza. Spain; ${ }^{(4)}$ CIBERSAM. Instituto de Salud Carlos III. Madrid. Spain; ${ }^{(5)}$ Department of Public Health. Faculty of Medicine and Health Sciences. Ghent University. Ghent. Belgium; ${ }^{(6)}$ Section for Epidemiology and Social Medicine (EPSO). Sahlgrenska Academy. University of Gothenburg. Gothenburg. Sweden; ${ }^{(7)}$ Institute of Food Sciences. CNR. Avellino. Italy.

Introduction: The global prevalence of children with overweight/obesity has increased significantly in the last 30 years. The IDEFICS study was a behavioural intervention implemented in eight European countries with the aim of preventing obesity in children.

Objetives: To compare whether the effectiveness of the intervention on the Body Mass Index (BMI) z-score varied between intervention and control groups according to a social vulnerability score.

Methods: Data from 8,365 children was included in the analysis. The vulnerability score was computed by adding the number of vulnerabilities a child had been exposed to at baseline. We defined a total of 6 vulnerabilities: minimal parental social network, non-traditional family structure, at least one non-native parent, at least one parent unemployed, low parental educational level and low household income. We performed the t-student test to compare BMI z-score mean differences (follow-up BMI z-score mean minus baseline BMI z-score mean) between intervention and control groups for each vulnerability score category.

Results: In girls with 3-6 vulnerabilities, the BMI z-score mean difference was significantly different between the intervention and control groups (mean difference $\pm S D: 0.06 \pm 0.57$ versus mean difference $\pm S D: 0.21 \pm 0.59$, respectively; $\mathrm{p}=0.009$; Cohen's $\mathrm{d}=0.26$ ). In girls exposed to 2 vulnerabilities, the BMI z-score mean difference was statistically different between the intervention and control groups (mean difference \pm SD:0.04 \pm 0.62 versus mean difference $\pm S D: 0.20 \pm 0.64$, respectively; $p=0.003$; Cohen's $d=0.25$ ). In boys exposed to 1 vulnerability, the BMI z-score mean difference was significantly different between the intervention and control groups (mean difference \pm SD:0.08 \pm 0.80 versus mean difference $\pm S D: 0.17 \pm 0.58$, respectively; $p=0.029$, Cohen's $d=0.13$ ).

Conclusions: Girls with 2 or more vulnerabilities and boys with 1 vulnerability in the intervention group had a better BMI outcome than their control group counterparts. Besides classical socio-economical indicators (education and income), other social vulnerabilities may be influencing intervention outcomes. We recommend that obesity prevention programmes collect sufficient data to continue evaluating these relationships in order to improve future intervention programmes.

Conflict of interest: Authors declare no conflict of interest.

Key words: obesity / children / social-vulnerability / prevention programme.

\section{5 \\ RECRUITMENT RATE IN A KINDERGAR- TEN-BASED, FAMILY-INVOLVED INTERVENTION TO PREVENT CHILDHOOD OBESITY ACROSS EU- ROPE BY SOCIO-ECONOMIC STATUS: THE TOY- BOX STUDY}

\author{
${ }^{(1,2,3)}$ P. De Miguel-Etayo; ${ }^{(3)}$ C. Summerbell; ${ }^{(4)} \mathrm{O}$. Androutsos; ${ }^{(5)} \mathrm{G}$. \\ Cardon; ${ }^{(6)}$ N. Usheva; ${ }^{(7)}$ P. Socha; ${ }^{(6)}$ Y. Manios; ${ }^{(1,2,8)}$ L.A. Moreno.
}

\begin{abstract}
${ }^{(1)} G r o w t h$, Exercise, NUtrition and Development (GENUD) Research Group. Instituto Agroalimentario de Aragón (IA2). Instituto de Investigación Sanitaria Aragón (IIS Aragón). University of Zaragoza. Zaragoza. Spain; ${ }^{(2)}$ CIBERobn Fisiopatología de la Obesidad y Nutrición. Instituto de Salud Carlos III. Madrid. Spain; ${ }^{(3)}$ Faculty of Social Sciences and Health. Durham University. Durham. United Kingdom; ${ }^{(4)}$ Department of Nutrition and Dietetics. School of Health Sciences \& Education. Harokopio University. Athens. Greece; ${ }^{(5)}$ Department of Movement and Sport Sciences. Ghent University. Ghent. Belgium; ${ }^{(6)}$ Department of Paediatrics. Medical University Varna. Varna. Bulgaria; ${ }^{(7)}$ The Children's Memorial Health Institute. Warsaw. Poland; ${ }^{(8)}$ Department of Physiatry and Nursing. Faculty of Health Sciences. University of Zaragoza. Zaragoza. Spain.
\end{abstract}

Introduction: There is strong evidence that obesity prevention interventions should focus on young children and their families for greatest impact and strive to rebalance inequalities in health which we see across Europe. The ToyBox Intervention aims to prevent obesity in preschool children and their families, and was developed using an evidence-based approach. The implementation was conducted within one academic year in a kindergarten setting and family and home environment.

Objectives: To assess the difference in recruitment rate of the ToyBox Intervention in kindergartens across Europe depending on their socio-economic status (SES). 
Methods: ToyBox is a cluster-randomised study that started in 2010 and included children aged 3.5-5.5 years and their families from six European countries. In each country, tertiles were derived from considering schools in three SES groups based on mean years of education or annual income.

Results: In total, 1,003 schools were approached (344 low, 311 medium and 348 high SES status) by mail or email and/or meeting with head teachers in all countries. $30.9 \%$ of kindergartens responded to our invitations to participate in the ToyBox study. Of this sample, 111 kindergartens were low, 104 were medium, and 96 were high SES status. The highest acceptance rate was observed in Bulgaria (70.8\%). Of those kindergartens who took part in the study, $60.6 \%$ were randomised to the intervention group. No significant difference in acceptance to take part in the study was observed by SES status of the kindergarten, or by country or study group.

Conclusions: In conclusion, the Toybox Intervention was deemed successful in its ability to reach kindergarten children and their families in low SES areas compared with medium or high SES areas, regardless of country or study group. Specific factors associated with this success should be translated and adopted for other public health interventions across Europe.

Conflict of interest: Authors declare no conflict of interest.

Key words: process-evaluation / recruitment / inequalities / preschoolers / obesity.

\section{6}

\section{ARE VITAMIN B12 AND B9 AND TOTAL HOMO- CYSTEINE CONCENTRATIONS THE SAME IN OLDER ADULTS WITH DIFFERENT LEVELS OF PHYSICAL FITNESS?}

\author{
${ }^{(1)}$ R. Aparicio-Ugarriza; (1) O. López-Torres; ${ }^{(1,2)}$ G. Palacios; ${ }^{(2,3)} A$. \\ Julibert; ${ }^{(2,3)}$ M.M. Bibiloni; ${ }^{(1,2)}$ M. González-Gross. \\ (1)ImFINE Research Group. Faculty of Physical Activity and \\ Sport Science. Health and Human Performance Department. \\ Universidad Politécnica de Madrid. Madrid. Spain; ${ }^{(2)}$ CIBERobn \\ Physiopathology of Obesity and Nutrition. Instituto de Salud \\ Carlos III. Madrid. Spain; ${ }^{(3)}$ Research Group on Community \\ Nutrition and Oxidative Stress (NUCOX). Fundamental Biology \\ and Health Science. University of the Balearic Islands. Spain.
}

Introduction: The analysis of blood markers allows the assessment of the response of the human body at different levels of physical fitness (PF).

Objectives: To evaluate the association between PF levels and being or not within the reference range of several biomarkers in older Spanish adults.

Methods: A cross-sectional study from the PHYSMED project was carried out in 429 participants (43\% males) aged over 55 years. Males and females were classified according to their PF level (low, medium and high) following a battery of PF tests (aerobic capacity, agility and dynamic balance, leg strength and handgrip strength). Fasting blood samples (vitamin B12 (B12), folates and total homocysteine (tHcy)) were collected and participants were also grouped depending on whether a particular biomarker was out of or in its reference range. Dietary and drug intake were considered as covariables using two non-consecutive $24 \mathrm{~h}$ dietary recalls and a specific questionnaire, respectively. A generalized linear model was used to examine the relationship between PF levels and biomarkers.

Results: Higher values out of the reference range were obtained for tHcy (24.3\%) and subclinical B12 (51.8\%). Participants in the low PF group showed higher B12 levels within the reference range $(\mathrm{p}<0.05)$. Nevertheless, participants in the low PF group displayed higher tHcy levels out of the reference range than in the high PF group $(\mathrm{p}<0.05) . \mathrm{B} 12$ and tHcy levels out of the reference range (low B12 and high tHcy) increased significantly $(\mathrm{p}<0.05)$ with advanced age in males.

Conclusions: A high percentage of participants presented concentrations out of their reference range for B12 and tHcy, which requires particular attention. Different behaviours of biomarkers were observed between PF levels. Further studies are required to determine how being out of the reference range can affect health in the long term.

Acknowledgements: Supported by Instituto Salud Carlos III (PI11/01791 \& CB12/03/30038).

Conflict of interest: Authors declare no conflict of interest.

Key words: elderly / biomarkers / vitamins / drug / diet.

\section{7 \\ HIGHER ADHERENCE TO THE MEDITERRANEAN DIET ENTAILS LOWER CYBERBULLYING BEHAV- IOUR: THE MEDIATING ROLE OF SOCIAL COM- PETENCE}

\author{
${ }^{(1)}$ N. Ruiz; ${ }^{(2)}$ A. Zaragoza; ${ }^{(1)}$ R. Ferrer; ${ }^{(1)}$ N. Albaladejo, ${ }^{(1)}$ M. Sánchez; \\ ${ }^{(1)} \mathrm{V}$. Clement. \\ (1)Departamento de Psicología de la Salud. Universidad de \\ Alicante. Alicante. Spain; ${ }^{(2)}$ Departamento de Enfermería. \\ Universidad de Alicante. Alicante. Spain.
}

Introduction: Dietary patterns have been previously related to social behaviour in several populations. In this regard, recent studies have demonstrated that poorer dietary outcomes are significantly related to dysfunctional relationships in school settings, such as bullying and cyberbullying. Hence, it has recently been found that poor nutrition significantly increases the probability of bullying behaviours in adolescents. However, no studies have analysed the association between healthy dietary patterns, such as the Mediterranean Diet (MD) and other types of bullying, such as cyberbullying. Moreover, possible interpersonal mediators in this relationship, such as social competence, have not previously been identified.

Objectives: To analyse the relationship between the MD, cyberbullying behavior and social competence.

Methods: A cross-sectional study was conducted with 527 adolescents from public secondary schools in a Mediterranean 
region in Spain. Adherence to the MD was evaluated through the KIDMED questionnaire, cyberbullying was evaluated with the E-Bullying Scale and social competence with the Personal Strengths Inventory-2(PSI-2).

Results: The obtained results showed a significant negative association between higher MD adherence and cyberbullying behaviour, and a positive association between MD adherence and social competence. Regression analyses exhibited a full mediation effect of social competence in the relationship between MD adherence and lower rates of cyberbullying behaviour.

Conclusions: The results of the present study indicate a possible protective effect of the MD on cyberbullying behaviour through the mediation role of social competence. The adequate intake of specific nutrients directly related to the adaptive neurological functioning and mood state could explain the protective effects of the MD on bullying behaviours. However, future studies should identify other potential mechanisms that could explain the association between the MD and cyberbullying.

Conflict of interest: Authors declare no conflict of interest.

Key words: Mediterranean diet / cyberbullying / social competence / adolescents.

\section{8 \\ SKIN TEMPERATURE RESPONSE TO A LIQUID MEAL INTAKE IN ADULTS: THE ROLE OF GENDER}

\author{
L. Ortiz-Alvarez; B. Martinez-Tellez; G. Sanchez-Delgado; H. Xu; F. \\ Acosta; E. Merchan-Ramirez; J. Ruiz.
}

Departamento de Educación Física y Deportiva. Facultad de Ciencias del Deporte. Universidad de Granada. Granada. Spain.

Introduction: The thermic effect of food (TEF) refers to the increase in energy expenditure and body temperature in response to food ingestion. To date, studies have focused on determining the TEF in terms of energy expenditure, but little is known about the response in terms of skin temperature.

Objectives: To assess whether the TEF on the skin temperature after a standardized and individualized liquid meal test differs between men and women.

Methods: A total of 104 young adults (66\% women, 18-25 years of age) consumed a standardized and individualized liquid meal (energy intake: $50 \%$ of measured basal metabolic rate, $47 \%$ carbohydrates, $35 \%$ fat, $15 \%$ protein, $3 \%$ fiber). The skin temperature was measured by 17 wireless thermometers (iButtons) for 3 hours and 20 minutes after the intake of the meal. We calculated the mean, proximal, distal, and supraclavicular skin temperature, as well as the level of peripheral vasoconstriction using the homemade software Temperatus $\subseteq$. The participants reported the thermal sensation of the whole body, and clavicular, feet, and hand zones along the duration of the test.

Results: Mean, proximal, and supraclavicular skin temperature significantly increased after the meal intake (all $\mathrm{P}<0.05)$. There was a postprandial peripheral vasoconstriction just after the meal intake and for the first hour, and a peripheral vasodilatation for the

XVII Conference of SEÑ and X Meeting of ACCA second and third hour. Women presented a higher increase in all skin temperature parameters in comparison to men (all $\mathrm{P}<0.05$ ), except for proximal skin temperature $(\mathrm{P}=0.279)$. The pattern of thermal sensation after the intake was similar between genders, but women always felt colder than men.

Conclusions: A standardized and individualized liquid meal test increased the skin temperature in young adults, the thermic effect being higher in women than in men. These findings suggest that the thermoregulatory system is more effective in women than in men.

Conflict of interest: Authors declare no conflict of interest.

Key words: meal-induced thermogenesis / thermoregulation / body temperature / thermal preception.

\section{9 \\ DIFFERENCES IN GUT MICROBIOTA COMPOSI- TION DEPENDING ON THE ALCOHOL CONSUMP- TION PATTERN IN HEALTHY SPANISH ADULTS}

\author{
N. Redondo; A. Gheorghe; L.E. Diaz; B. Villavisencio; A. Marcos; E. \\ Nova.
}

Departamento de Metabolismo y Nutrición. Instituto de Ciencia y Tecnología de los Alimentos y Nutrición (ICTAN-CSIC). Madrid. Spain.

Introduction: Acute alcohol consumption has been related to intestinal dysbiosis in animals, but evidence in healthy adults is scarce.

Objectives: To assess the influence of alcohol consumption patterns on gut microbiota (GM) of healthy Spanish adults.

Methods: An observational study was performed on 261 subjects (25-45 y) (49\% women). Alcohol consumption habits (frequency, amount and pattern) of different drinks were registered in a specific questionnaire, and three groups were considered: 1) None-Low ( $\mathrm{n}=66$ ), $<5 \mathrm{~g}$ alcohol/day; 2$)$ Moderate-Regular ( $\mathrm{n}=98)$ : $\geq 5 \mathrm{~g} / \mathrm{d}$ and $\leq 20 \mathrm{~g} / \mathrm{d}$ (men) or $\leq 12 \mathrm{~g} / \mathrm{d}$ (women) and no excessive alcohol at the weekend or at least 3 days/wk when average intake $<5 \mathrm{~g} / \mathrm{d}$; 3) Excessive consumption at the weekend (EWEND) ( $n=21): \geq 50 \mathrm{~g} / \mathrm{d}$ for men and $\geq 30 \mathrm{~g} / \mathrm{d}$ for women plus different patterns at the weekend from working days. GM was analyzed through $16 S$ rRNA sequencing and taxa identification by comparative rRNA taxonomy. After data cleansing and matching for confounder factors, bacterial differences among groups were analysed by Kruskal-Wallis and Mann-Whitney tests. q-values $<0.05$ using the Storey method were considered significant.

Results: The EWEND group showed higher relative abundances of Fusobacteria, as well as Actinoallumurus ( $\mathrm{q}=0.032)$, Bulleida $(\mathrm{q}=0.010)$, Gramella $(\mathrm{q}=0.018)$ and Candidatus Amoebophilus $(\mathrm{q}=0.028)$ compared to the moderate-regular group $(\mathrm{q}=0.025)$.

Conclusions: The alcohol consumption patterns did not show association with the main phyla and genera, but changes in minor bacterial groups ( $<\% 5$ abundance) suggest that acute alcohol consumption could affect the GM to a greater extent than moderate and regular consumption habits. 
Conflict of interest: Authors declare no conflict of interest. Key words: alcohol / gut microbiota / healthy adults.

\section{0 \\ DAIRY CONSUMPTION AND DIET QUALITY IN- DEX IN A SAMPLE OF EUROPEAN CHILDREN}

${ }^{(1)}$ E.M. Gonzalez-Gil; (1)A.M. Santaliestra-Pasías; ${ }^{(2)}$ V. Pala; ${ }^{(3)}$ T. Intemann; ${ }^{(3)}$ A. Hebestreit; ${ }^{(4)}$ P. Russo; ${ }^{(5)}$ C. Van Aart; ${ }^{(1)}$ L.A. Moreno.

\begin{abstract}
${ }^{(1)}$ GENUD (Growth, Exercise, NUtrition and Development) Research Group. Facultad de Ciencias de la Salud. Universidad De Zaragoza. Spain; (2)Department of Research. Epidemiology and Prevention Unit. Fondazione IRCCS Istituto Nazionale dei Tumori. Milan. Italy; ${ }^{(3)}$ Leibniz Institute for Prevention Research and Epidemiology - BIPS. Bremen. Germany; ${ }^{(4)}$ Department of Public Health. Ghent University. Ghent. Belgium; ${ }^{\left({ }^{5}\right)}$ Department of Pharmacological Sciences. University of Milan. Milan. Italy.
\end{abstract}

Introduction: Consumption of dairy products has been related with health benefits, although the associations remain unclear. Also, it seems that the type of the dairy product is relevant in this association due to variable macro and micronutrients. Finally, consumption of dairy products could be related with diet quality.

Objectives: To assess the association of dairy products with the Diet Quality Index (DQI) in a sample of European children.

Methods: 1688 children from the IDEFICS study were included in the analysis ( $50.8 \%$ boys) measured at baseline and after two years. A food frequency questionnaire (FFQ) was used to calculate the DQI. The DQI was used as a proxy to ascertain the diet quality of the children and consisted of three components: dietary quality, dietary diversity and dietary equilibrium. Milk, yogurt and cheese were assessed individually and in combination for dairy consumption. Linear regression analysis was performed to assess this association.

Results: In the linear regression analysis significant associations were found between dairy consumption and DQI $(\mathrm{p}<0.05)$ in the total sample, in boys, and in girls at both measurement time points (adjusted for BMI z-score, age and SES). Associations with DQI were found for milk + yogurt, and milk + yogurt + cheese, being positive and significant by gender and at both measurement time points.

Conclusions: This association suggests that consumption of dairy products is related with a higher DQI in children. This relationship was higher for the combination of dairy products, i.e. milk + yogurt + cheese. More studies are needed to assess the associations between dairy products and lifestyle behaviours.

Conflict of interest: Authors declare no conflict of interest.

Key words: dairy / diet quality / children / Europe.
011

\section{MAIN MEAL ACCOMPANIMENT INFLUENCES FOOD AND BEVERAGE CONSUMPTION IN EU- ROPEAN ADOLESCENTS: THE HELENA STUDY}

\author{
(1)A.M. Santaliestra-Pasias; (1)A. Perez Felez; ${ }^{(2)}$ I. Huybrechts; ${ }^{(3)}$ L. Censi; \\ ${ }^{(4)}$ M. Gonzalez-Gross; ${ }^{(5)}$ M. Forsner; ${ }^{(6)}$ M. Sjostrom; ${ }^{(1)}$ L.A. Moreno. \\ (1)Fisiatria y Enfermería. Universidad de Zaragoza. Zaragoza. \\ Spain; ${ }^{(2)}$ International Agency for Research on Cancer. Lyon. \\ France; ${ }^{(3)}$ CREA Research Centre for Food and Nutrition. Rome. \\ Italy; ${ }^{(4)}$ Health and Human Performance. Universidad Politécnica \\ de Madrid. Madrid. Spain; ${ }^{(5)}$ Nursing. Umeå University. Sweden; \\ ${ }^{\left({ }^{(6)} B i o s c i e n c e s ~ a n d ~ N u t r i t i o n ~ a t ~ N O V U M . ~ K a r o l i n s k a l n s t i t u t e t . ~\right.}$ \\ Huddinge. Sweden.
}

Introduction: The social environment, especially accompaniment at main meals, influences adolescent behaviours regarding food and beverage consumption.

Objectives: To assess the relationship between accompaniment during main meal occasions and food and beverage consumption in European adolescents.

Methods: Adolescents from the European HELENA cross-sectional study were selected $(n=1,703$ adolescents aged 12.5-17.5 years (46.5\% males)). Socio-demographic variables and dietary intake using two non-consecutive self-reported 24hour dietary recalls $(24 \mathrm{H}-\mathrm{DR})$ were collected. The accompaniment (by family, friends, other people or alone) during the main meal occasions (breakfast, lunch and dinner) was registered through the $24 \mathrm{H}-\mathrm{DR}$. The relationship between the social environment at meal times and consumption of foods and beverages was analysed using mixed models with random effects for country, adjusted by the mother's education, body mass index z-score and total energy intake.

Results: Adolescents who usually had their main meals with their family (reference group) were associated with a high consumption of healthy foods and beverages (i.e. vegetables, fruit, milk, water) and low consumption of energy-dense food and beverages such as chocolate, savoury snacks, sugar or juices compared with those who usually ate alone, with friends or other people $(\mathrm{p}<0.05)$. Those who usually had their lunch alone had a low consumption of fruits $(\beta-27.9$ (95\%CI: $-52.18 ;-3.81))$ and water ( $\beta-174.8$ (95\%CI: $-307.7 ;-42.01))$ in boys, and cereals $(\beta-16.8$ (95\%CI: $-32.02 ;-1.70)$ ) in girls, compared with those who usually had lunch with their family. Also, those who usually had dinner with their friends had a low consumption of fruits $(\beta-47.1$ (95\%CI:-42.70;-0.61)) and milk ( $\beta$-76.0 (95\% CI -143.9;-8.17)) in boys, and high consumption of sugars ( $\beta$ 18.2 (95\%CI:6.76;29.74)) and nuts ( $\beta 4.22$ (95\% CI: 0.72;7.72) in girls compared with those who had lunch with their family.

Conclusions: A family environment at meals has been associated with a high consumption of healthy foods.

Conflict of interest: Authors declare no conflict of interest.

Key words: accompaniment / food / beverages / adolescent / HELENA. 


\section{2}

\section{MEAL-INDUCED THERMOGENESIS IN META- BOLICALLY HEALTHY BUT OBESE ADULTS}

\author{
H. Xu; G. Sanchez-Delgado; B. Martinez-Tellez; V. Muñoz-Hernandez; \\ W.D. Martinez-Avila; F.J. Amaro-Gahete; J. R. Ruiz.
}

Departamento de Educación Física y Deportiva. Facultad de Ciencias del Deporte. Universidad de Granada. Granada. Spain.

Introduction: In simple terms, obesity is the consequence of a positive energy balance (i.e. energy intake exceeding energy expenditure), among other factors. There is a subgroup of obese patients that present a healthy metabolic profile, the so-called metabolically healthy but obese (MHO) phenotype. One component of the total energy expenditure is meal-induced thermogenesis (MIT), which refers to the increase in energy expenditure after a single meal. Currently, it is unknown whether MHO individuals differ in MIT from their metabolically unhealthy and obese (MUO) peers.

Objectives: To determine whether the MIT in response to a single standardized liquid meal is different in MHO vs. MUO.

Methods: We assessed the basal metabolic rate (BMR) for a standardized 30 minutes and following a strict experimental control in 16 young adults classified as MHO (62.5\% women) and 8 young adults classified as MUO (37.5\% women). We also assessed MIT after a standardized and individualized liquid meal (energy intake: $50 \%$ of measured BMR, $47 \%$ carbohydrates, $35 \%$ fat, $15 \%$ protein and 3\% fiber), using indirect calorimetry (MedGraphics Inc.) during 3 periods of 1 hour each. We calculated the area under the curve (AUC) of the MIT following the trapezoidal rule.

Results: The BMR was similar in $\mathrm{MHO}$ and $\mathrm{MUO}(\mathrm{P}=0.657)$. The MIT was slightly higher in MHO during the 1st and 2nd period of measurement, whereas during the $3 \mathrm{rd}$ period it was very similar between phenotypes $(\mathrm{P}$ for time $=0.270$; $\mathrm{P}$ for interaction=0.092). Furthermore, we found that the AUC of the total MIT was higher in MHO vs. MUO $(\mathrm{P}=0.012)$.

Conclusions: We show for the first time that MIT is higher in $\mathrm{MHO}$, which could partially explain the healthy characteristics of this phenotype.

Conflict of interest: Authors declare no conflict of interest. Key words: BMR / BAT / TEF / IC / metabolic-syndrome.

013

\section{RELATIONSHIP BETWEEN CALORIC INTAKE AND HEALTH-RELATED QUALITY OF LIFE IN THE ELDERLY}

\author{
(1) A. Zaragoza; (2) R. Ferrer; (1)J.A. Hurtado; (1) A. Laguna; (1)M.J. \\ Cabañero; ${ }^{(3)}$ M.F. Zaragioza; ${ }^{(2)}$ N. Ruiz.
}

(1)Departamento de enfermería. Universidad de Alicante. Spain; ${ }^{(2)}$ Departamento de psicología de la salud. Universidad de Alicante. Spain; ${ }^{(3)}$ Departamento de Estudios Jurídicos del Estado. Universidad de Alicante. Spain.

XVII Conference of SEN and X Meeting of ACCA
Introduction: Eating habits are directly related to the health status of the population. In particular diets with a low caloric intake are related to greater longevity and a lower risk of chronic-degenerative diseases. Nowadays, there is an excessive consumption of food and this is reflected in the numerous health problems that are appearing, being more pronounced in the elderly.

Objectives: To evaluate the relationship between caloric intake and health-related quality of life (HRQoL) in people over 60 years old.

Methods: A transversal descriptive study was carried out with 340 subjects older than 60 years and resident in the Spanish Mediterranean. All subjects with a score of 3 or more errors in the Pfeiffer test were excluded. The caloric intake was measured using the MEDIS-FFQ Food Consumption Frequency Questionnaire validated for this study population and the HRQoL was obtained with the SF-12.

Results: The average intake of calories was $2222.48 \mathrm{Kcal}$, with a minimum intake of $1271.25 \mathrm{Kcal}$ and with a maximum intake of 2995.50. The correlation analysis shows an inverse correlation between caloric intake and HRQoL $(\mathrm{r}=-0.78, \mathrm{p}<0.05)$, indicating that the higher the caloric intake, the lower the HRQoL. The results were slightly more significant in women than in men.

Conclusions: The results show a clear relationship between caloric intake and health-related quality of life, as subjects with a higher caloric intake had a lower HRQoL. More food-oriented policies are therefore needed to identify food-related risk factors in order to develop better programs for the promotion of health.

Conflict of interest: Authors declare no conflict of interest.

Key words: nutrition / quality of life / elderly / food habits / energy intake.

\section{4}

\section{BEVERAGE CONSUMPTION ASSOCIATED WITH BODY COMPOSITION AND THE RISK OF CHRON- IC NON-COMMUNICABLE DISEASES IN ADULTS}

\author{
${ }^{(1)}$ A.L. López-Santana; ${ }^{(1)}$ E.H. Valdés Miramontes; ${ }^{(2)}$ P.J. López-Uriarte. \\ (1)Centro de Investigaciones en Comportamiento Alimentario \\ y Nutrición. México; ${ }^{(2)}$ Centro de Investigación en Biología \\ Molecular de las Enfermedades Crónicas (CIBIMEC). Universidad \\ de Guadalajara. Mexico.
}

Introduction: Chronic non-communicable diseases (CNCDs) are a worldwide public health problem. In Mexico, the main causes of death are cardiovascular diseases and DM2. One of the factors associated with obesity and its comorbidities is the consumption of caloric beverages, mainly soft drinks. However, the daily caloric contribution provided by the different types of drinks consumed by a group of adults is unknown.

Objectives: To determine the beverage consumption pattern in different stages of adulthood and its association with weight, body composition and the risk of developing CNCDs.

Methods: A quantitative questionnaire (Beverage Questionnaire) was applied to estimate the caloric intake from beverages, 
by quantity, frequency and type of beverage consumed, to know the pattern and habits of consumption in adults. Physical activity level was determined (IPAQ questionnaire) and a clinical history was applied. Waist circumference, height and weight were measured and body composition was determined by bioelectrical impedance.

Results: Through a cross-sectional, observational and analytical study, 119 individuals (20-80 years) of both sexes were evaluated, grouped into four stages of adulthood. Of the total, $54 \%$ were overweight or obese. Although all adults exceeded the caloric intake from beverages, the young adults had the highest calorie consumption from all beverages $(p=0.02)$, sweetened $(p=0.50)$ and non-sweetened beverages $(\mathrm{p}=0.00)$. A negative correlation was observed between the consumption of non-sweetened beverages and the variables of weight, waist and body composition $\mathrm{p}(<0.05)$.

Conclusions: Although the sweetened drink intake provided high amounts of calories/day, the energy from other beverages, such as alcoholic beverages, contributed more to the caloric intake observed and thus the increased risk to health. The beverage consumption differed between the stages of life, with young people presenting the most non-adequate intakes.

Conflict of interest: Authors declare no conflict of interest.

Key words: consumption / beverages / body composition / adults.

\section{5}

\section{HEPATIC STATUS AND ADHERENCE TO A MEDI- TERRANEAN DIETARY PATTERN IN OBESE SUB- JECTS WITH NON-ALCOHOLIC FATTY LIVER DIS- EASE}

\author{
${ }^{(1)}$ C. Galarregui; ${ }^{(1,2)}$ I. Abete; ${ }^{(1)}$ I. Cantero; ${ }^{(3,4)}$ J.I. Monreal; ${ }^{(3,4)}$ M. Elorz; \\ ${ }^{(3,4)}$ A. Benito-Boillos; ${ }^{(1,2)}$ J.A. Martínez; ${ }^{(1,2)}$ M.A. Zulet.
}

(1)Departamento de Ciencias de la Alimentación y Fisiología. Universidad de Navarra. Pamplona. Spain; ${ }^{(2)}$ CIBERobn, ISCIII. Madrid. Spain; ${ }^{(3)}$ IdiSNA. Pamplona. Spain; ${ }^{(4)}$ Clínica Universidad de Navarra. Pamplona. Spain.

Introduction: The interplay between liver status and nutrition has important health implications. Furthermore, overall diet quality measurements have been proposed as a suitable tool to evaluate diet-disease relationships.

Objectives: To evaluate the relationship between adherence to a Mediterranean diet (MedDiet) pattern and variables related to liver status in obese subjects with non-alcoholic fatty liver disease (NAFLD).

Methods: One hundred and twenty-two adults (age: 51.2 \pm 9.1 years old; BMI: $33.9 \pm 4 \mathrm{~kg} / \mathrm{m}^{2}$ ) with NAFLD assessed by abdominal ultrasonography and Magnetic Resonance Imaging were analyzed. Adherence to the MedDiet was assessed with a Mediterranean Diet Score (MDscore $\leq 8$ low adherence). Anthropometrics, blood pressure, body composition (DXA), glycemic and lipid profile, $\mathrm{C}$-reactive protein and liver non-invasive biomarkers and scores were measured at baseline. This study was registered as FLiO: Fatty Liver in Obesity study; NCT03183193.

Results: Subjects showed low adherence to the MedDiet according to the MDscore $(6.08+2.15)$. Negative correlations between the MDscore and hepatic fat measured by Magnetic Resonance Imaging $(\mathrm{r}=-0.2965, \mathrm{p}<0.01)$ and $\mathrm{C}$-reactive protein $(\mathrm{r}=-0.203, \mathrm{p}<0.05)$ were found. Additionally, there was a positive correlation of MedDiet adherence with HDL-c levels $(\mathrm{r}=0.19, \mathrm{p}<0.05)$. Patients with better adherence (MDscore $>6$ ) had significantly lower values in android fat, $\mathrm{HbA1c}$ and $\mathrm{C}$-reactive protein as well as significantly higher HDL-c levels ( $\mathrm{p}<0.05$ for all comparisons) than those with lower MedDiet adherence (MDscore $<6$ ). Furthermore, positive correlations between hepatic fat and GGT $(\mathrm{r}=0.34, \mathrm{p}<0.001)$, AST $(\mathrm{r}=0.40, \mathrm{p}<0.001) \mathrm{TG}(\mathrm{r}=0.19, \mathrm{p}<0.05)$, glucose $(\mathrm{r}=0.23, \mathrm{p}<0.05)$, C-reactive protein $(r=0.22, p<0.05)$, APRI $(r=0.36, p<0.001)$ and FLI $(r=0.38, p<0.001)$ indexes were observed.

Conclusions: Greater adherence to a MedDiet pattern is associated with lower values of hepatic fat and related outcomes, which could be considered as an appropriate healthy dietary pattern in the management and prevention of NAFLD.

Conflict of interest: Authors declare no conflict of interest.

Key words: Mediterranean diet / NAFLD / obesity.

\section{6}

\section{SHOULD FRESH MEAT AND MEAT DERIVATIVES BE PLACED AT DIFFERENT LEVELS OF THE NU- TRITIONAL PYRAMID?}

\section{S. Sánchez-Pérez; O. Comas-Basté; S. Hernández-Macias; N.C. Muñoz-Esparza; M.L. Latorre-Moratalla; M.T. Veciana-Nogués; M.C. Vidal-Carou.}

Departament de Nutrició, Ciències de l'Alimentació i Gastronomia. Facultat de Farmàcia i Ciències de l'Alimentació. INSA.UB. XaRTA. Universitat de Barcelona. Santa Coloma de Gramenet. Spain.

Introduction: Meat and meat products are good source of proteins and iron, but also of fats, saturated fats and salt, which are extensively related with cardiovascular diseases. They are usually placed at the same level of the nutritional pyramid, but some doubts have arisen about the suitability of this positioning, considering their contents of salt, fat and saturated fat.

Objectives: To estimate the current intake of total fat, saturated fat, salt and iron from meat and meat products and to evaluate if they should be separated in the nutritional pyramid.

Methods: A total of 243 products were considered: fresh meat (beef and pork) and meat derivatives (dry-fermented sausages and cured and cooked ham), selected as the most consumed according to MAPAMA ${ }^{1}$. Composition data of fresh meat came from the $\mathrm{FEN}^{2}$ and the data of the meat derivatives from the labels of commercial products. The ENALIA $2^{3}$ data was used for the intake estimation.

Results: According to ENALIA data, the mean consumption of fresh meat (beef and pork) is $80.3 \mathrm{~g} /$ day and that of meat deriva- 
tives $29.8 \mathrm{~g} /$ day. The current consumption of meat derivatives represents $10 \%$ of the recommended maximum fat intake and $12 \%$ of saturated fat. These contributions are twice those from fresh meat. Regarding salt, consumption of meat derivatives contributes more than $20 \%$ to the maximum set by OMS4. In contrast, consumption of fresh meat provides $9 \%$ of the iron RDI, which is more than double the amount provided by meat derivatives (4\%).

Conclusions: The estimated intake of fat, saturated fat and salt by the consumption of dry-fermented sausages and cured and cooked ham confirms the current recommendation to moderate their consumption and supports placing them separately from fresh meat in nutritional pyramids.

References: ${ }^{1}$ Ministerio de Agricultura, Pesca, Alimentación y Medio Ambiente (2016); ${ }^{2}$ Fundación Española de la Nutrición (2009); ${ }^{3}$ Encuesta Nacional de Alimentación (2015).

Conflict of interest: Authors declare no conflict of interest.

Key words: fat / saturated fat / salt / iron / meat.

\section{7}

\section{INSUFFICIENT INTAKE OF VITAMINS IN BREAST- FEEDING MEXICAN MOTHERS AT TWO MONTHS POSTPARTUM}

\author{
${ }^{(1)}$ N.C. Muñoz-Esparza; ${ }^{(2)}$ E.M. Vásquez-Garibay; ${ }^{(2)}$ E. Guzmán- \\ Mercado; ${ }^{(2)}$ A. Larrosa-Haro; E. Romero-Velarde; ${ }^{(1)}$ M.C. Vidal-Carou. \\ (1)Departament de Nutrició, Ciències de l'Alimentació i \\ Gastronomia. Facultat de Farmàcia i Ciències de l'Alimentació. \\ INSA.UB. XaRTA. Universitat de Barcelona. Santa Coloma de \\ Gramenet. Spain; ${ }^{(2)}$ Institute of Human Nutrition. University of \\ Guadalajara. Guadalajara. Mexico.
}

Introduction: During the postpartum period, women undergo physiological, endocrine, nutritional and psychological changes. It has been observed that during this period women are more susceptible to vitamin deficiencies, which are associated with an increased risk of postpartum depression. Also, it has been observed that the diet can influence the content of vitamins in human milk. Therefore, the nutritional requirements of breastfeeding mothers are increased by the metabolic effort involved in milk production.

Objectives: To evaluate if mothers who are exclusively breastfeeding have an adequate intake of vitamins at two months postpartum.

Methods: A cross-sectional study, with non-random sampling, included 219 mothers from the Nuevo Hospital Civil de Guadalajara, who met inclusion criteria and signed the informed consent. A $42 \%$ of the mothers exclusively breastfed, $36.5 \%$ partially-breastfed and $21.5 \%$ used milk-based infant formula. A 24 hour reminder survey was conducted at two months postpartum. The intake of liposoluble and water-soluble vitamins and adequacy percentages were estimated (Nutrikcal ${ }^{\circledR}$ ) according to the Recommended Daily Ingestion (Institute of Medicine,2011). The protocol was approved by the Research and Bioethics Committee of the hospital and financed by CONACyT-234158.

Results: Only half of the total mothers had an adequate intake of thiamine, cobalamin and riboflavin. An insufficient intake

XVII Conference of SEÑ and X Meeting of ACCA
( $<80 \%$ of the requirements) was observed in $95 \%$ of mothers in vitamin-E, folic acid (93\%), pyridoxine (75\%), vitamin-A (74\%) and vitamin-C (61\%). No significant differences were found among groups.

Conclusions: The results show an insufficient vitamin intake in most mothers. Although the nutritional requirements in lactating women increase significantly, there were no differences in the intake of vitamins in comparison with other mothers. This is a worrying finding, due to the implications of this deficit on the health of the mother and the content of vitamins in human milk, which may eventually affect the growth and development of the infant.

Conflict of interest: Authors declare no conflict of interest. Key words: exclusive breastfeeding / lactating women / infant. 


\section{Poster Abstract Presentations}

\section{8 \\ OBESE ADULT MALE AND FEMALE MICE SHOW BEHAVIOR, IMMUNE FUNCTION AND REDOX IMPAIRMENT AS WELL AS A SHORTER LIFE SPAN}

C. Hunsche; I. Martínez de Toda; B. Jiménez; N. Ceprián; M. De La Fuente.

Department of Genetics, Physiology and Microbiology (Animal Physiology Unit). Faculty of Biology. Complutense University of Madrid. Madrid. Spain.

Introduction: Obesity has been linked to alterations in behavior and immune function. We have demonstrated that the early onset of obesity in juvenile female mice resulted in a premature immunosenescence at the adult age, and this condition was aggravated throughout the aging process.

Objectives: Here, we investigated whether the later onset of obesity could affect relevant behavioral, immunological and oxidative stress parameters as well as the life span of male and female mice.

Methods: A total of 32 adult male and female ICR/CD1 mice ( 28 weeks old, $n=8$ per group) were fed either a high-fat diet ( $60 \%$ of fat) or a standard diet for 14 weeks. After that, when animals were late-adults (42 weeks old), a battery of behavioral tests were performed, and blood and peritoneal leukocytes were obtained in order to analyze biochemical, immunological and oxidative stress parameters. The same parameters (excepting behavioral tests) were repeated when female mice were old (72 weeks old).

Results: The results showed that obese late-adult mice from both sexes presented higher values of triglycerides and cholesterol, higher anxiety-like behavior, lower neuromuscular vigor and exploratory activity than non-obese mice. Obese late-adult mice also showed lower values of immune functions (chemotaxis of macrophages and lymphocytes, phagocytosis of macrophages, NK antitumor activity and lymphoproliferative response to mitogens) and of antioxidant defenses (catalase activity and GSH levels) as well as higher values of oxidants (xanthine oxidase activity, malondialdehyde levels and GSSG/GSH ratios) than late-adult controls. Sex differences were found in some behavioral and immunological parameters, as well as in the life span, males being significantly more affected than females. With aging, old obese female mice continue to exhibit greater deterioration in the parameters studied than non-obese old mice.

Conclusions: In conclusion, late-adults with obesity show impaired behavior, immunity and oxidative stress, which impair their longevity.

Conflict of interest: Authors declare no conflict of interest.

Acknowledgements: FIS(PI15/01787)(ISCIII-FEDER).

Key words: obesity / immunosenescence / oxidative stress / mice.

32

Ann Nutr Metab 2018;73 (suppl 2): 1-93 DOI: $10.1159 / 000490752$
019

DEGREE OF ADHERENCE TO THE MEDITERRANEAN DIET OF SPANISH POSTGRADUATE STUDENTS IN THE FIELD OF FOOD

\author{
${ }^{(1)}$ I. Elío; ${ }^{(1)}$ S. Sumalla; ${ }^{(1)}$ M. Hernández; ${ }^{(1)}$ M. Montserrat; ${ }^{(1)}$ L. Bravo- \\ Lamas; ${ }^{(2)}$ M. Battino. \\ (1)Departamento de Alimentación. Universidad Europea del \\ Atlántico. Santander. Spain; ${ }^{(2)}$ Director Científico de la Facultad \\ de Ciencias de la Salud de la Universidad Europea del Atlántico. \\ Santander. Spain.
}

Introduction: Eating habits are closely related to health.

Objectives: To determine the dietary food pattern by the degree of adaptation to the Mediterranean Diet (DM).

Methods: A descriptive cross-sectional study was carried out with the collaboration of Spanish postgraduate students in the food area of FUNIBER. The participants were selected by accidental non-probabilistic sampling. They filled out a sociodemographic questionnaire and a 3-day dietary record. The sample consisted of 100 students. The records were evaluated with the NutrIber software and the data obtained were analyzed with the statistical package SPSS V.22.0.

Results: The profile of the students was $74 \%$ women, with an average age of $36.6(+/-10.5)$ years, and a body mass index of 22.6 $\mathrm{kg} / \mathrm{m}^{2}(+/-3.3)$. The consumption of fruit was deficient in $79 \%$, and vegetables deficient in $68 \%$. Bread, pasta, rice, couscous and other cereals were acceptable in $46 \%$; dairy products deficient in $69 \%$; nuts, seeds and olives deficient in $85 \%$; potatoes deficient in $65 \%$; white meat excessive in $64 \%$, red meat excessive in $68 \%$, processed meats excessive in 49\%; fish and shellfish optimal in $85 \%$; eggs deficient in $49 \%$; legumes deficient in $84 \%$; sweets excessive in $76 \%$.

Conclusions: Postgraduate students of this study had moved away from the DM model, with a diet deficient in fruit, vegetables, olive oil, dairy products, nuts, potatoes, eggs and legumes. In contrast, they had an excessive intake of meat (white, red, processed) and sweets.

Conflict of interest: Authors declare no conflict of interest.

Key words: Mediterranean diet / postgraduate students / FUNIBER.

\section{0}

\section{INCREASE OF OXIDATIVE STRESS IN ADULT PREMATURELY AGING MICE FED WITH A GLIA- DIN-ENRICHED DIET}

\footnotetext{
${ }^{(1,2)}$ M. De la Fuente; ${ }^{(1,2)}$ A. Garrido; ${ }^{(3)} Y$. Sanz; ${ }^{(1)}$ E. Díaz-Del Cerro; ${ }^{(1,2)} N$. Ceprian.
}

(1)Department of Genetics, Physiology and Microbiology (Animal Physiology Unit). Faculty of Biology. Complutense University of Madrid. Madrid. Spain; ${ }^{(2)}$ Institute of Investigation of Hospital 12 de Octubre (i+12). Madrid. Spain; ${ }^{(3)}$ Microbial Ecology, Nutrition 
\& Health Research Group. Institute of Agrochemistry and Food Technology. National Research Council (IATA-CSIC). Valencia. Spain.

Introduction: Aging has as a base an oxidative stress (increase of oxidants and decrease of antioxidants). Adult prematurely aging mice (PAM), a model of premature aging based on the inadequate response to a stress situation, which exhibit premature immunosenescence, oxidative stress and a shorter life span with respect to their counterparts of the same chronological age (NPAM, non-prematurely aging mice), have been proposed as animals with a possible susceptibility to celiac disease. Previous studies have shown that PAM exposed in vitro and in vivo to gliadin, peptic fragments that cause the development of celiac disease, exhibited altered immune functions similar to those with this condition.

Objectives: To determine the possible effects of a gliadin-enriched diet on the redox state of PAM.

Methods: Adult female ICR-CD1 mice were classified as PAM and NPAM. PAM were divided into two groups ( $\mathrm{N}=7$ per group). One group was fed a gliadin-enriched diet (PAMGLU, $120 \mathrm{~g} / \mathrm{kg}$ diet), and the other a standard diet (PAMC) for 4 weeks. After that, the lung, liver, heart, kidney, spleen and thymus were obtained and several antioxidants and oxidants were analyzed in these organs and in leukocytes isolated from spleen and thymus. As antioxidant defenses, the activity of enzymes such as glutathione peroxidase $(\mathrm{GPx})$ and glutathione reductase (GR) as well as the concentrations of glutathione reduced (GSH) were measured. As oxidants, xanthine oxidase (XO) activity and oxidized glutathione (GSSG) concentrations were evaluated.

Results: The results showed that PAMGLU exhibited lower GPx (thymus: $p=0.000$; liver: $p=0.017$; lung: $p=0.005$; kidney: $\mathrm{p}=0.013$; spleen: $\mathrm{p}=0.006$; heart: $\mathrm{p}=0.004$ ), $\mathrm{GR}$ activities (thymus: $\mathrm{p}=0.031$; liver $\mathrm{p}=0.005$; lung $\mathrm{p}=0.02$; kidney: $\mathrm{p}=0.029$; spleen: $\mathrm{p}=0.005$ ), and GSH concentrations (spleen and thymus: $\mathrm{p}=0.003$; liver $\mathrm{p}=0.017$; lung: $\mathrm{p}=0.005$; kidney: $\mathrm{p}=0.004$ ) as well as higher XO activity (lung: $p=0.035$; spleen: $p=0.027$; heart: $p=0.007$ ) and GSSG concentrations (liver: $\mathrm{p}=0.002$; kidney: $\mathrm{p}=0.004$; spleen: $\mathrm{p}=0.025$ ), with respect to those in PAMC.

Conclusions: In conclusion, the ingestion of a gliadin-enriched diet in PAM causes an oxidative stress and consequently accelerates their aging process.

Acknowledgements: FIS (PI15/01787) (ISCIII-FEDER).

Conflict of interest: Authors declare no conflict of interest.

Key words: gliadin-enriched diet / PAM / oxidative stress.

\section{1}

\section{PLENUFAR 6: STUDY OF PHYSICAL ACTIVITY PRACTICE AND DIET QUALITY BY A COMMUNI- TY PHARMACY}

\author{
A. Garcia; A. Bach-Faig; F. Jaraíz; P. León; M.J. Moreno-Aliaga; MR. \\ Pastor.
}

National Committee of Food Pharmacists. General Pharmaceutical Council of Spain. Madrid. Spain.
Introduction: Regular physical exercise, supported by a correct diet, is a basic pillar of health and quality of life. In 2017 the General Pharmaceutical Council of Spain organized the Plenufar 6 campaign with the aim of promoting both aspects. During the campaign a study was carried out among users of the Community Pharmacy.

Objectives: To know the eating habits and physical exercise practice of the surveyed population.

Methods: 11.342 anonymous surveys were conducted by 4,200 participating pharmacists. The survey included a validated questionnaire to assess diet quality, as well as a questionnaire to analyse the intensity of physical activity by users.

Results: $50.2 \%$ of the users surveyed performed vigorous physical activity ( $\geq 6 \mathrm{METs}-\mathrm{h} /$ day), which was slightly more intense in males (9.2 \pm 7.2 vs. $7.2 \pm 6.3 \mathrm{METs}-\mathrm{h} /$ day). Despite this, the figures for overweight and obesity were high, at $30.6 \%$ and $8.8 \%$, respectively. More than $50 \%$ of the respondents took some nutritional supplement to encourage exercise practice and subsequent recovery. Only $15.3 \%$ of people had an adequate diet based on quality, and $61.3 \%$ had areas that could be improved. On the other hand, 23.4\% had a diet with significant imbalances.

Conclusion: The Community Pharmacist can help to encourage physical exercise among users and provide dietary guidelines to maximize the beneficial effects of this exercise.

Conflict of interest: Authors declare no conflict of interest.

Key words: physical activity / diet / exercise.

\section{2 \\ REDUCING SUGAR CONSUMPTION: A PUBLIC HEALTH PRIORITY IN CATALONIA}

\author{
M. Manera; G. Salvador; C. Castell; C. Cabezas.
}

Agència de Salut Pública de Catalunya (ASPCAT). Barcelona. Spain.

Introduction: THE WHO warns that free sugar intake increases the risk of obesity, tooth decay and non-communicable diseases. Therefore, its reduction is an important part of health promotion and prevention strategies.

Objectives: To describe the actions of the ASPCAT (Agència de Salut Pública de Catalunya) in relation to sugar reduction, through:

- Incorporating a clear recommendation to reduce sugar consumption in documents addressed to professionals, companies and the general public.

- Supporting fiscal measures to implement a tax on packaged sugary beverages (IBEE).

Methods: Scientific evidence was reviewed, working groups were established and recommendations made and disseminated in paper and online documents, emails, social networks, lectures and workshops.

Institutional and technical support was given for the design and application of the IBEE.

Results: In the last 6 documents addressed to children and in 2 to adults, the recommendation of "the less, the better" was in- 
corporated for foods rich in free sugars, and examples were given of meals free of these foods. This recommendation was also made in the 3 editions of the online course on dietary advice in childhood (1300 participants) and in the 42 lectures and workshops addressed to 2500 primary care and school professionals.

Law 5/2017 on the creation and regulation of the IBEE was enacted and put in place by the Economy Department with technical support of the ASPCAT.

The document "Facts about the IBEE tax" includes answers to the questions (FAQs) generated by this measure, as well as about sugars and health.

Conclusions: The recommendations issued in the healthy food documents and workshops of the ASPCAT, and the application of the law 5/2017 show a clear position in relation to the recommendation to reduce sugar consumption.

Conflict of interest: Authors declare no conflict of interest.

Key words: sugar / TAX / public health / obesity.

\section{3 \\ TRIPLE BURDEN OF HYPERLIPIDEMIA, OVER- WEIGHT AND SARCOPENIA IN A SUBPOPULA- TION OF CUBAN ELDERS.}

\author{
${ }^{(1)} H$. Hernández-Hernández; ${ }^{(2)}$ M.E. Díaz-Sánchez; ${ }^{(1)}$ M. Díaz- \\ Domínguez; ${ }^{(1)}$ Ruiz-Álvarez V, . Ruiz-Álvarez; ${ }^{(1)} Y$. Lanyau- \\ Domínguez; ${ }^{(2)}$ Y. Díaz-Fuentes; ${ }^{(2)} \mathrm{C}$. Roque-Treville; ${ }^{\left({ }^{2}\right)}$ Y. Vasallo-Key. \\ (1)Departament of Biochemistry and Physiology. Center for \\ Nutrition and Food Hygiene. National Institute for Hygiene, \\ Epidemiology and Microbiology. Havana. Cuba; ${ }^{(2)}$ Departament \\ of Comunity Nutrition. Center for Nutrition and Food Hygiene. \\ National Institute for Hygiene, Epidemiology and Microbiology. \\ Havana. Cuba.
}

Introduction: Several conditions related to nutrition coincide during late adulthood; their detection allows proper nutritional interventions so they can be controlled and the life quality of the elderly improved.

Objectives: To evaluate the prevalence of overweight, sarcopenia and hyperlipidemia in a sample of elders from Havana, and describe how their distribution is related.

Methods: A cross-sectional study was carried out in 115 elders (mean age $74.8 \pm 9.29$ years), who were submitted to anthropometric (weight, height, BMI, muscle mass and strength) and metabolic (blood glucose, triglycerides, and cholesterol: total, HDL, and LDL) measurements using conventional methods. Participants were classed as under-, normal- and overweight according to WHO criteria for BMI; they were also identified as normolipemic and hyperlipemic, and sarcopenic and non-sarcopenic, according to the respective reference criteria. Descriptive statistical analyses were performed.

Results: Among all the subjects, $86.9 \%$ showed hyperlipidemia, $56.5 \%$ overweight and $28.7 \%$ sarcopenia. Those with LDL had the highest frequencies of risk levels (76.5\%) followed by total cholesterol (45.2\%). Normal- and underweight individuals represented $37.4 \%$ and $6.1 \%$, respectively of the subjects. Most (69.2\%) of the overweight subjects were pre-obese. There was a high prevalence of hyperlipidemia both in those who were overweight $(92.3 \%)$ or not $(80 \%)$, and a higher proportion of sarcopenia in the latter $(50 \%)$ than the former $(12.3 \%)$. In the group with sarcopenia, $24.2 \%$ were overweight compared with $69.5 \%$ in the non-sarcopenic group. Both groups exhibited high frequencies of hyperlipidemia: $86.6 \%$ and $87.9 \%$, respectively. LDL was the most altered lipid in every group.

Conclusions: The high prevalence of hyperlipidemia pinpoints the need to test for this condition regardless of the elder's weight or presence of sarcopenia. The distribution of sarcopenia suggests its assessment is of particular importance in elders who are not overweight. Nutritional interventions are needed to reduce the prevalence of hyperlipidemia, overweight, and also sarcopenia in elderly populations.

Conflict of interest: Authors declare no conflict of interest.

Key words: elders/ hyperlipidemia/ overweight/ sarcopenia/ prevalence.

\section{4 \\ RELATIONSHIP BETWEEN POLYPHENOLS AND BODY WEIGHT IN ADOLESCENTS: A PILOT STUDY}

${ }^{(1)}$ E.P. Laveriano-Santos; ${ }^{(2,3)} A$. Treserra-Rimbau; ${ }^{(3,4)}$ M. Domenech; ${ }^{(3,4)}$ R. Estruch; ${ }^{(5,6)}$ G. Santos-Beneit; ${ }^{(6)}$ J.M. Fernández-Alvira; ${ }^{(6,7)} \mathrm{V}$. Fuster; ${ }^{(1,3)}$ R.M. Lamuela-Raventós.

(1)Department of Nutrition, Food Science and Gastronomy. School of Pharmacy and Food Sciences. XaRTA. INSA. University of Barcelona. Barcelona. Spain; ${ }^{(2)}$ University Hospital of Sant Joan de Reus. Department of Biochemistry and Biotechnology. Faculty of Medicine and Health Sciences. Universitat Rovira i Virgili. Reus. Spain; ${ }^{(3)}$ Biomedical Research Networking CenterPhysiopathology of Obesity and Nutrition (CIBEROBN). Carlos III Health Institute (ISCIII). Government of Spain. Madrid. Spain; ${ }^{(4)}$ Department of Internal Medicine. Hospital Clínic. Institut $d^{\prime}$ Investigacions Biomèdiques August Pi i Sunyer (IDIBAPS). University of Barcelona. Barcelona. Spain; ${ }^{(5)}$ Foundation for Science, Health and Education (SHE). Barcelona, Spain; ${ }^{(6)}$ Centro Nacional de Investigaciones Cardiovasculares Carlos III (CNIC). Madrid. Spain; ${ }^{(7)}$ The Zena and Michael A. Wiener CVI. Icahn School of Medicine at Mount Sinai. New York. USA.

Introduction: Overweight and obesity have become a major risk factor in human health, increasing the probability of cardiovascular diseases. The relationship between polyphenols and body weight was recently studied in a population of adolescents.

Objectives: To analyze the relationship between total urinary polyphenol excretion (TPE), as a biomarker of total polyphenol intake, and body weight in a pilot study from the SI! Secondary Program performed in adolescents aged 11-12 years.

Methods: In a cross-sectional study of 44 participants ( 18 boys and 26 girls), trained personnel performed anthropometric meas- 
ures (height, weight, waist circumference and body fat). Height and weight were used to calculate the body mass index (BMI), while body fat was estimated using bioelectrical impedance. We used the Folin-Ciocalteu colorimetric method, assisted by solid phase extraction, to determine the TPE in urine samples taken before breakfast. TPE was categorized into tertiles $(\mathrm{T} 1<12.04, \mathrm{~T} 2$ of 12.04-16.18 and T3>16.18 mg gallic acid equivalent in urine/g creatinine). Multiple linear regression models were used to assess the relationship between TPE with anthropometric measures, adjusting for sex and age.

Results: Participants in the highest tertile of TPE had a body weight $49.3 \pm 13.1 \mathrm{~kg}$, BMI $19.7 \pm 4.9 \mathrm{~kg} / \mathrm{m}^{2}$, waist circumference $67.0 \pm 10.5 \mathrm{~cm}$, and body fat $26.2 \pm 7.4 \%$. Significant inverse association was observed between TPE and body weight $(b=-18,936$; $\mathrm{P}=0,036)$, BMI $(\mathrm{b}=-6.362 ; \mathrm{P}=0.017)$, waist circumference $(\mathrm{b}=-$ $16,706 ; \mathrm{P}=0.026)$, and body fat $(\mathrm{b}=-4.870, \mathrm{P}=0.021)$.

Conclusions: Higher total urinary polyphenol excretion is related to a better body weight in adolescents.

Conflict of interest: Authors declare no conflict of interest.

Key words: polyphenol / adolescents / body weight / bmi.

\section{5 \\ WELL-BEING IS ASSOCIATED WITH BODY MASS INDEX, EATING AND HEALTH-RELATED BEHAV- IORS AMONG YOUNG ADULTS}

\author{
${ }^{(1)}$ M.F. Zeron-Rugerio; ${ }^{(1)}$ A. Farran-Codina; ${ }^{(2)}$ A. Díez-Noguera; ${ }^{(2)}$ T. \\ Cambra; ${ }^{(1)}$ M. Izquierdo-Pulido. \\ (1)Department of Nutrition, Food Science and Gastronomy. \\ School of Pharmacy and Food Science. University of Barcelona. \\ Spain; ${ }^{(2)}$ Department of Biochemistry and Physiology. School of \\ Pharmacy and Food Science. University of Barcelona. Spain.
}

Introduction: Subjective well-being (SWB), the individual appraisal or evaluation of a person's own life, has been found to be related to health behaviors and disease outcomes. However, little is known about the association between SWB in young adults and body mass index (BMI), eating behaviors (EB) (the cognitive-behavioral nature of food intake), and health-related behaviors such as adherence to the Mediterranean diet (AtMD), sleep quality (SQ) and physical activity (PA).

Objective: To study the potential association between SWB and BMI, EB, AtMD, SQ and PA in young adults.

Methodology: Three hundred and forty-eight students from the University of Barcelona were included in a cross-sectional study. The Well-being Index (WHO-5) was used to assess SWB, the Three Factor Eating Behavior Questionnaire (TFEQ-21) to assess the three dimensions of EB (emotional (EE), uncontrolled (UE) and/or restraint eating (RE)), the KIDMED questionnaire for AtMD, the Pittsburg Sleep Quality Index (PSQI) for SQ, and the short version of the International Physical Activity Questionnaire (IPAQ) for PA. Higher scores indicated higher SWB, EB and AtMD, vigorous PA, and a poor SQ. Data were collected using an Open Data Kit. Statical analysis was adjusted for confounding variables.

XVII Conference of SEÑ and X Meeting of ACCA
Results: The mean score of the WHO-5 was lower in individuals with $\mathrm{BMI}>25 \mathrm{~kg} / \mathrm{m}^{2}(\mathrm{p}<0.05)$. The WHO-5 index was negatively associated with EE $(\mathrm{p}=0.000), \mathrm{UE}(\mathrm{p}=0.015)$ and PSQI $(\mathrm{p}=0.000)$, and positively associated with the KIDMED questionnaire $(\mathrm{p}=0.000)$ and IPAQ $(\mathrm{p}=0.006)$.

Conclusion: Our findings suggest that in a young population, SWB is higher in normal-weight than in overweight or obese subjects. Additionally, higher SWB is associated with certain eating and health behaviors, such as a lower EE and UE, as well as a higher AtMD, a good SQ and being more physically active. Therefore, SWB is associated with healthy behaviors in young adults.

Conflict of interest: Authors declare no conflict of interest. Key words: well-being / sleep quality / diet / BMI.

\section{6 \\ SOCIAL JETLAG AND EVENINGNESS IS ASSO- CIATED WITH BODY MASS INDEX AND ADHER- ENCE TO THE MEDITERRANEAN DIET IN YOUNG ADULTS}

\author{
${ }^{(1)}$ M.F. Zeron-Rugerio; ${ }^{(1)}$ A. Farran-Codina; ${ }^{(2)}$ A. Díez-Noguera; ${ }^{(2)}$ T. \\ Cambra; ${ }^{(1)}$ M. Izquierdo-Pulido. \\ (1)Department of Nutrition, Food Science and Gastronomy. \\ School of Pharmacy and Food Science. University of Barcelona. \\ Spain; (2)Department of Biochemistry and Physiology. School of \\ Pharmacy and Food Science. University of Barcelona. Spain.
}

Introduction: Social jetlag, the discrepancy between biological and social timing, results in a misaligned circadian system linked to a higher body mass index (BMI) and unhealthy dietary patterns in adolescents and middle-aged adults. Moreover, social jetlag tends to be greater in individuals with either a low sleep quality or evening preferences, which has been also associated with obesity and metabolic syndrome. However, the association between social jetlag and adherence to the Mediterranean diet has been relatively unexplored.

Objective: To examine associations among social jetlag, eveningness, sleep quality, BMI, and adherence to the Mediterranean Diet in young adults.

Methods: Five hundred and thirty-eight students from the University of Barcelona were included in a cross-sectional study. Social jetlag (SJL) was assessed calculating the difference between the midpoint of sleep on free days and workdays. Chronotype was assessed using the Horne and Östgeberg's morningness-eveningness questionnaire (MEQ), sleep quality with the Pittsburg Sleep Quality Index (SQ) and adherence to the Mediterranean Diet (AtMD) with the KIDMED questionnaire. Data was collected using an Open Data Kit and statistical analysis was performed using SPSSv24.

Results: The average of SJL was $1.35 \pm 0.91$ hours. Positive associations were found between SJL, PSQI $(\mathrm{p}<0.001)$ and BMI $(\mathrm{p}<0.05)$, while negative associations were found between $\mathrm{SJL}$, KIDMED $(\mathrm{p}<0.001)$ and MEQ $(\mathrm{p}<0.001)$, as well as MEQ and BMI $(\mathrm{p}<0.05)$ and PSQI and KIDMED $(\mathrm{p}<0.001)$. 
Conclusions: Young adults tend to go to bed 1.35 hours later on free days. We observed that greater SJL was associated with higher BMI, eveningness and a lower SQ and lower AtMD. Preference towards eveningness was associated with higher BMI in young adults, while a low SQ was associated with a low AtMD. Within this context, sleep timing and quality as well as chronotype emerge as modifiable risk factors for obesity in young adults.

Conflict of interest: Authors declare no conflict of interest.

Key words: social jetlag / chronotype / diet / BMI.

\section{7}

\section{PLACENTAL LIPID DROPLET COMPOSITION IS ASSOCIATED WITH MATERNAL CLINICAL PA- RAMETERS AND CORD BLOOD METABOLITES BUT NOT WITH MATERNAL DIET}

\author{
${ }^{(1)}$ A. Gázquez; ${ }^{(1)} O$. Uhl; (2) M. Ruíz-Palacios; ${ }^{(3)}$ C. Gill; ${ }^{(3)} N$. Patel; ${ }^{(2)}$ E. \\ Larqué; ${ }^{(1)} B$. Koletzko; ${ }^{(3)}$ L. Poston. \\ ${ }^{(1)}$ Division of Metabolic and Nutritional Medicine. Dr. von \\ Hauner Children's Hospital. Ludwig Maximilian University of \\ Munich. Munich. Germany; ${ }^{(2)}$ Department of Physiology. Faculty \\ of Biology. University of Murcia. Murcia. Spain; ${ }^{(3)}$ Division of \\ Women's Health. Women's Health Academic Centre. King's \\ College London. St. Thomas' Hospital. London. United Kingdom.
}

Introduction: Lipid droplets (LD) are intracellular structures implicated in storage and hydrolysis of neutral lipids. However, their role in placental tissue is poorly understood.

Objectives: To investigate LD composition in placentas from obese women and associations with maternal and infant parameters.

Methods: LD were isolated from placentas of 43 obese pregnant women randomized to a behavioural intervention designed to improve glycaemic control (intervention; $n=20$, control; $n=23$ ) (UPBEAT trial). Triglycerides (TG) and cholesterol in LD were quantified by commercial kits. Total FA, phosphatidylcholines (PC) and sphingomyelins (SM) in LD and cord blood were analyzed by UPLC-MS/MS. Differences between groups were assessed by t-test and associations to clinical parameters by Pearson correlation.

Results: Lipid droplet TG, cholesterol, FA, PC and SM composition were not different between randomized groups. Placental weight was associated with more saturated PC and less polyunsaturated FA (PUFA) in LD (particularly arachidonic and docosahexaenoic (DHA) acids). The intervention changed the dietary glycaemic index $(53.0 \pm 1.1$ vs. $57.9 \pm 0.9, \mathrm{p}=0.001)$ and the glycaemic index correlated inversely with \% DHA in LD $(\mathrm{R}=-0.32$, $\mathrm{p}=0.04)$. Dihomo- $\gamma$-linolenic acid was positively associated with gestational weight gain. PUFA and PUFA-containing complex lipids in placental LD were positively correlated with cord blood lipids (e.g. DHA: $\mathrm{R}=0.42$, $\mathrm{p}=0.01$; PC.18:0/20:4: $\mathrm{R}=0.70, \mathrm{p}<0.001$ ).

Conclusions: A lifestyle intervention which improved diet and reduced gestational weight gain in obese pregnant women did not modify LD metabolic profiles. Placental LD PUFA appear to be a source of cord blood PUFA and could play a role in regulation of fetal growth.

Conflict of interest: Authors declare no conflict of interest. Keys words: placenta / lipids / pregnancy / intervention / obesity.

\section{8 \\ SERUM FATTY ACID COMPOSITION IN SPANISH VEGETARIANS}

\section{Pilar Vaquero; A.M. Salvador; A. Gallego-Narbón; B. Zapatera}

Department of Metabolism and Nutrition. Institute of Food Science, Technology and Nutrition (ICTAN-CSIC). Madrid. Spain.

Introduction: Plant food-based diets have become progressively common in developed countries, including Spain. However, information on the nutritional status of vegetarians according to biochemical determinations is lacking.

Objectives: The aim of this study was to characterise the fatty acid profile of a Spanish vegetarian population.

Methods: The design was a cross-sectional observational pilot study. Healthy adult vegetarians were recruited in the Madrid region ( $\mathrm{n}=104,83$ women, 21 men). According to the type of diet, 49 were ovo-lacto vegetarians ( 11 men and 38 women) and 55 vegans (12 men and 43 women). Food intake and the use of supplements were assessed by a food frequency questionnaire and blood samples were collected and stored. Serum fatty acids were analysed by gas chromatography.

Results: Fatty acid profiles did not vary significantly between vegetarians and vegans, or in relation to gender. Percentages of fatty acids of the whole studied population were $27.5 \%$ saturated (SFA), $24.6 \%$ monounsaturated (MUFA), and $47.9 \%$ polyunsaturated (PUFA) of which $45.2 \%$ were omega- 6 (n-6) and $2.7 \%$ omega-3 (n-3). The main serum fatty acids were linoleic acid $(34.4 \pm$ $4.4 \%)$, oleic acid $(23.4 \pm 5.6 \%)$, palmitic acid (19.2 $\pm 2.3 \%)$, and arachidonic acid $(8.3 \pm 1.8 \%)$. A high PUFA $n-6 / n-3$ ratio (19:1) was found in this population. Consumers of $n-3$ supplements $(n=9,10 \%$ of the population) compared to non-consumers presented a significantly lower serum $n-6 / n-3$ ratio, $14: 1$ compared to 19:1 ( $\mathrm{p}=0.025)$, due to significantly higher serum $\mathrm{n}-3$ levels $(3.7 \%$ compared to $2.6 \%, \mathrm{p}=0.003$ ) and a tendency to lower $\mathrm{n}-6$ levels (42.6\% compared to $45.5 \%$ ).

Conclusions: Vegetarians whose diets are n-3-supplemented presented a more adequate serum lipid profile with higher PUFA n-3 levels and n-6/n-3 ratio.

Acknowledgements: Research project was financed by Zamdeh laboratories.

Conflict of interest: Authors declare no conflict of interest.

Key words: serum fatty acids / vegetarians / vegans / n6/n3-ratio 


\section{9}

\section{VITAMIN B12 STATUS OF SPANISH VEGETARI- ANS DETERMINED BY COBALAMIN AND METH- YLMALONIC ACID SERUM LEVELS}

\author{
M.P. Vaquero; A. Gallego-Narbón; B. Zapatera.
}

Department of Metabolism and Nutrition. Institute of Food

Science, Technology and Nutrition (ICTAN-CSIC). Madrid. Spain.

Introduction: Vitamin-B12 deficiency is a common health issue in vegetarians, and more so in vegans, if fortified foods or supplements are not consumed. There is no ideal diagnostic marker of vitamin-B12 deficiency, as the single determination of the total serum vitamin levels usually does not rule out functional deficiency conditions. Methylmalonic acid (MMA), an intermediate of the one-carbon metabolism in which vitamin-B12 participates as a cofactor, increases prior to the vitamin-B12 decrease and is a good biomarker of the status of this vitamin.

Objectives: To assess for the first time the vitamin-B12 status of Spanish vegetarians, studied by both serum MMA and vitamin-B12. In addition, to examine the influence of dietary preferences (ovo-lacto vegetarian or vegan) and the intake of cobalamin supplements.

Methods: Healthy vegetarian adults $(n=104)$ were recruited. Dietary patterns and consumption of supplements were assessed by questionnaires, and blood samples were collected. Serum vitamin-B12 was measured by chemiluminiscence and serum MMA by liquid chromatography tandem mass spectrometry (LC-MS/MS).

Results: The obtained median (IQR) values were: vitamin-B12, 280.4 (160.2) pmol/l, and MMA, 139.9 (78.9) nmol/l. There were no differences associated with gender, age or the type of vegetarian diet. The consideration of vitamin-B12 levels alone resulted in the detection of 2 deficient individuals, while the addition of MMA as a second marker identified a $10 \%$ of mildly deficient subjects. Consumption of vitamin-B12 supplements by $75 \%$ of the participants was associated with higher vitamin-B12 $(\mathrm{p}<0.001)$ and lower MMA $(\mathrm{p}=0.012)$ levels compared to non-consumption.

Conclusions: The studied Spanish lacto-ovo vegetarians and vegans presented a generally sufficient cobalamin status, which can be explained by an extended awareness of the need for supplement consumption.

Conflict of interest: Authors declare no conflict of interest.

Acknowledgements: Research project financed by Zamdeh Laboratories.

Key words: methylmalonic acid / vitamin-B12 / vegetarian / vegan.

\section{0 \\ MEDIATING ROLE OF ADOLESCENT BODY WEIGHT ON THE RELATIONSHIP BETWEEN SO- CIOECONOMIC STATUS AND ADOLESCENT MENTAL HEALTH: THE LSAC STUDY}

\author{
${ }^{(1)}$ I. Iguacel; (2)A. Chung; ${ }^{(2)}$ E. Gearon; ${ }^{(1)}$ L. Moreno; ${ }^{(2)}$ A. Peeters; ${ }^{(3)}$ S. \\ Petersen; ${ }^{(2)}$ K. Backholer. \\ (1)Departamento de Fisiatría y Enfermería. Universidad de \\ Zaragoza. Zaragoza. Spain; ${ }^{(2)}$ Public Health and Preventive \\ Medicine. Deakin University. Melbourne. Australia; ${ }^{(3)}$ Public \\ Health and Clinical Medicine Units. Epidemiology and Global \\ Health. Umea University. Umea. Sweden.
}

Introduction: It has been estimated that $10-20 \%$ percent of adolescents in developed countries experience mental disorders. Among adolescents with a low socioeconomic position (SEP), the risk of mental health problems is greater compared to adolescents with a high SEP. One of the pathways through which SEP might affect adolescent mental health could be body mass index (BMI). Adolescents with a low SEP are also at higher risk for overweight and obesity compared to adolescents with higher SEP, and simultaneousy, overweight and obesity in youth have been associated with poorer mental health. To our knowledge, the extent to which BMI mediates the relationship between SEP and mental health problems in the adolescent population has not been formally examined.

Objectives: To estimate the mediating role of the BMI z-score at the age of 12-13 years in the relationship between SEP and mental health at the age of 14-15 years.

Methods: A total of 2186 children from the Longitudinal Study of Australian Children were examined. SEP was measured as a continuous composite of parents' education, occupation and income. Mental health problems were assessed by the Strengths and Difficulties Questionnaire (SDQ), which was completed by parents and teachers. HRQoL was assessed by the Pediatric Quality of Life Inventory (PedsQL), which was completed by the parents. The Product of Coefficients mediation method was used to quantify the contribution of adolescent BMI to the relationship between SEP and mental health.

Results: Each decreasing decile of SEP was associated with higher mental health problems at $14-15$ years. Around $20 \%$ of these differences in mental health problems could be explained by socioeconomic-based differences in children's BMI.

Conclusions: Interventions to reduce socioeconomic inequalities behind mental health problems during adolescence should promote the attainment of a healthy weight during childhood and adolescence.

Conflict of interest: Authors declare no conflict of interest.

Key words: mental health / obesity / adolescence. 


\section{1}

\section{ESTIMATION OF DIETARY PHENOL COMPOUND INTAKE AND MAJOR FOOD SOURCES IN A SPAN- ISH TEENAGE POPULATION: STUDY OF THE SI! PROGRAM}

\begin{abstract}
${ }^{(1)}$ S. Castro-Barquero; ${ }^{(2)} A$. Tresserra-Rimbau; ${ }^{(1)} M$. Doménech; $;{ }^{(3)}$ R.M. Lamuela-Raventós; ${ }^{\left({ }^{4}\right)}$ G. Santos-Beneit; ${ }^{(5)}$ J.M. Fernández-Alvira; $(5,6)$ V. Fuster; ${ }^{(1)}$ R. Estruch.

(1)Department of Internal Medicine. Hospital Clinic. Institut $\mathrm{d}^{\prime}$ Investigació Biomèdica August Pi i Sunyer. Barcelona. Spain; (2)Department of Biochemistry and Biotechnology. Faculty of Medicine and Health Sciences. Universitat Rovira i Virgili. Reus. Spain; ${ }^{(3)}$ Department of Nutrition, Food Science and Gastronomy. School of Pharmacy and Food Sciences. XaRTA. INSA. University of Barcelona. Barcelona. Spain; ${ }^{(4)}$ Foundation for Science, Health and Education (SHE). Barcelona. Spain; ${ }^{(5)}$ Centro Nacional de Investigaciones Cardiovasculares Carlos III (CNIC). Madrid. Spain. ${ }^{(6)}$ The Zena and Michael A. Wiener CVI. Icahn School of Medicine at Mount Sinai. New York. USA.
\end{abstract}

Introduction: Polyphenol effects are attributed to the dietary polyphenol intake. However, there is limited information about polyphenol intake in the teenage population.

Objectives: To estimate the food intake and dietary polyphenol intake among the cohort of the SI! Program study. Also, to correlate food consumption with intake of individual polyphenols, polyphenol subclasses and total polyphenols, identifying the major food sources of polyphenols in this population.

Methods: A total of 398 participants included in the study of the SI! Program at baseline, aged between 11 and 12 years, completed a 1-year food frequency questionnaire (FFQ). Dietary polyphenol intake was obtained by the 155 -item FFQ. Data on the polyphenol content in foods were obtained from the Phenol-Explorer database (www.phenol-explorer.eu).

Results: The mean of total polyphenol intake was $345 \pm 172$ $\mathrm{mg} /$ day $(147 \pm 83.8 \mathrm{mg} /$ day of flavonoids, $78.8 \pm 47.4 \mathrm{mg} /$ day of phenolic acids, $0.17 \pm 0.19 \mathrm{mg} /$ day of stilbenes, $46.7 \pm 26.0 \mathrm{mg} /$ day of lignans and $74.9 \pm 55.3 \mathrm{mg} /$ day of other polyphenols). Among flavonoids, the intake of $(+)$-catechin was higher than apigenin and luteonil $(18.1 \pm 11 ; 0.09 \pm 0.1$ and $7.40 \pm 9.6 \mathrm{mg} /$ day respectively). Among phenolic acids, the intake of vanilic acid was higher than ferulic, sinapic and p-coumaric acids $(20.5 \pm 20 ; 0.75 \pm 0.7$; $2.48 \pm 2.4$ and $0.84 \pm 0.4 \mathrm{mg} /$ day, respectively). Lignan pinoresinol intake was $15.2 \pm 7.4 \mathrm{mg} /$ day. Among tyrosol derivates, hydroxytyrosol and tyrosol intake was $15.2 \pm 7.4$ and $3.89 \pm 4.9 \mathrm{mg} /$ day, respectively.

Conclusions: In a group of Spanish teenagers, the main polyphenol subclasses ingested were flavanols, followed by hydroxycinnamic acid, lignans and tyrosol. The main dietary sources of polyphenols were fruits and juices, olives, extra virgin olive oil, cocoa and nuts.

Conflict of interest: Authors declare no conflict of interest.

Key words: polyphenols / adolescents / nutrition / dietary intake.

\section{2}

\section{FEEDING HABITS AND BODY IMAGE IN 15-16-YEAR-OLD STUDENTS}

\author{
V. Torres. \\ Unidad de Protección de la Salud. Área Sanitaria Norte de \\ Málaga. Hospital de Antequera. Antequera. Spain.
}

Introduction: Eating Disorders $(\mathrm{AD})$ in adolescents are characterized by an unusual attitude toward food and an exaggerated concern for physical appearance. Distortions of body image can generate a negative physical image and trigger an alteration of eating patterns. Without an early diagnosis, these situations can cause difficulties in adult life.

Objectives: To try to delimit related differentiated patterns and profiles that could be predictive of AD in adolescence.

Methods: A descriptive study was carried out based on an unvalidated questionnaire interview of 6 questions given to $884^{\text {th }}$ year students of Compulsory Secondary Education at two mixed schools in Antequera. Qualitative variables were summarized in frequencies and percentages. Physical features were differentiated from those related to personality.

Results: The results reflect the greater concern of adolescent girls for body image. The concern for what others think is also higher in girls $(60 \%)$ than boys $(25 \%)$. In contrast, the image students think others have of them was not correlated with gender but with personality (37.4-50.5\%).

Although the socio-economic and cultural index of both centers is medium-low, without serious problems of coexistence or absenteeism, marked differences appear in aspects of personality and body image.

Conclusions: A routine assessment of signs of suspected AD may be useful to facilitate early therapeutic actions. It would be necessary to study the family socio-educational level or the geographical location of the center to rule out possible biases.

Conflict of interest: Authors declare no conflict of interest.

Key words: health / eating disorders / adolescents.

\section{3 \\ DIET FOR DIABETICS: EXTENSION OF FOOD GROUPS USED TO CALCULATE RATIONS

\footnotetext{
${ }^{(1,2)}$ M. Ros Baró; ${ }^{(1,3)}$ M. Rivero Urgell; ${ }^{(1,4)}$ G. Sabater Sales; ${ }^{(5)}$ R. Aispuru

(1)GENA (Group of Studies of Nutrition and Feeding). Barcelona. Spain; ${ }^{(2)}$ Diabetics Association from Catalonia. Barcelona. Spain; ${ }^{(3)}$ Departamento Técnico. Laboratorios Ordesa. Barcelona. Spain; ${ }^{(4)}$ Departamento de Nutrición. Salengei. Barcelona. Spain; ${ }^{(5)}$ Departamento Médico. Ubikare. Vitória. Spain.
}

Introduction: Most guides for diabetics currently only differentiate six groups of food, which we have observed is insufficient. An extension in the classification is proposed to offer a more balanced guide for the specialists in this field. 
Objectives: To provide a better adjustment of the analytical parameters in the use of these food groups for diabetic patients. To obtain a better distribution of food groups for calculating rations, thereby avoiding problems of nutritional deficiencies and imbalance.

Methods: Food groups not currently represented in diabetic diet tables that may offer an increase in nutritional value for patients were evaluated. In a nutritional assessment of 15 days for N25 diabetic people (from the Diabetics Association of Catalonia), we included groups such as legumes, cereals of fast and slow absorption, and nuts. Menus with the changes were provided and the preprandial glycemia was assessed in the early morning. We used the guides of Fundación Alícia, FESNAD, SEDCA and the evaluation of the surveys and menu calibration tables of CESNID (University of Barcelona).

Results: We observed a higher food variability in diets and an improvement in nutritional deficiencies, as well as improvements in most of the analytical parameters of the illness. In two out of three patients a decrease of prandial post-glycaemia was observed with the changes made, which is an average of $50 \mathrm{mg} / \mathrm{dl}$ of glucose.

Conclusions: This contribution provides a more up-to-date and realistic methodology that could be useful for health professionals who work with diabetic patients. This is owing to its fast implementation, balance, easy comprehension and improved results.

Conflict of interest: Authors declare no conflict of interest.

Keys words: diet / diabetics / supplementation / food.

\section{4}

\section{DIFFERENCES IN LYMPHOCYTE, METABOLIC AND INFLAMMATORY MARKER EVOLUTION BE- TWEEN ANOREXIA NERVOSA PATIENTS WITH GOOD AND POOR TREATMENT RESPONSE}

\author{
${ }^{(1)}$ E. Nova; ${ }^{(1)}$ A. Elegido; ${ }^{(2)}$ P. Andrés; ${ }^{(1)}$ L.E. Díaz-Prieto; ${ }^{(2)}$ M. Graell; ${ }^{(1)} A$. \\ Marcos. \\ (1)Instituto de Ciencia y Tecnología de Alimentos y Nutrición \\ (ICTAN). CSIC. Madrid. Spain. ${ }^{(2)}$ Unidad de Psiquiatría y Psicología \\ Infanto Juvenil. Hospital Universitario Niño Jesús. Madrid. Spain.
}

Introduction: Anorexia nervosa (AN) is a mental disorder with variable individual response to treatment and a high rate of relapse during the first year. Immune and metabolic hormone levels reflect adaptive changes under the impaired nutritional status.

Objectives: To examine the differences in lymphocytes, metabolic and inflammation markers during one year in patients admitted for specialized in-patient/day treatment showing a final good and poor response to treatment, respectively.

Methods: 57 adolescents (12-17 y.) with AN admitted for first treatment were included. Clinical and biological variables were measured on admission (V0) and at one-, six- and 12-month visits (V1, V6 and V12). 27 patients complying with all 4 visits were analysed. Anthropometrical recovery ( $\mathrm{z}$ score BMI $>-1$ ), absence of amenorrhea, psychiatric pharmacotherapy and concern with body shape (BSQ score $<80$ ) at V12 were used to classify patients as good $(\mathrm{N}=13)$ or poor $(\mathrm{N}=14)$ treatment responders. A lineal mixed model was used to assess the effect of "visit" and "response to treatment" and its interaction on the biomarkers.

Results: The "response to treatment" factor was significant for the following markers: leukocytes, triiodothyronine, estradiol, IGF-1 and leptin (all lower in poor responders) and T helper lymphocytes percentage, cortisol, TNF-alpha and soluble leptin receptor (all higher in poor responders). No baseline differences were observed between responder groups in any biomarker except for IGF-1 and estradiol. No interactions were found between "response to treatment" and "visit", although adiponectin changes between V0 and V12 tended to show $(\mathrm{P}=0,089)$ an increase in poor responders and a decrease in good responders.

Conclusions: Biomarkers reflecting the malnutrition status of AN patients evolve differentially in good versus poor responders to multidisciplinary treatment, suggesting that their repeated evaluation every two months after intensive treatment would be useful to predict individual medium-term evolution and prevent relapses.

Conflict of interests: Authors declare no conflict of interest.

Key words: anorexia nervosa / biomarkers / treatment response.

\section{5 \\ CONTAGION OF LIFESTYLE HABITS TO RELA- TIVES OF INDIVIDUALS FOLLOWING A LIFE- STYLE INTERVENTION: THE PREDIMEDPLUS "HALO EFFECT"}

\author{
(1)M.D. Zomeño; (2,3) N. Babio; ${ }^{(3,4)}$ R. Estruch; $(3,5) X$. Pintó; $(3,6) J$. \\ Basora; ${ }^{(2,3)}$ J. Salas-Salvadó; $(1,3) A$. Goday; $(1,3) O$. Castañer. \\ (1)Departament Risc Cardiovascular i Nutrició. Institut Hospital \\ del Mar d'Investigacions Mèdiques (IMIM). Barcelona. Spain. \\ ${ }^{(2)}$ Departament de Bioquimica i Biotecnologia. Facultat de \\ Medicina i Ciències de la Salut. Universitat Rovira i Virgili. \\ Barcelona. Spain. ${ }^{(3)}$ CIBEROBN. Madrid. Spain. ${ }^{(4)}$ Hospital \\ Clínic. Universitat de Barcelona. Barcelona. Spain. ${ }^{(5)}$ Hospital \\ Universitari de Bellvitge. Barcelona. Spain. ${ }^{\left({ }^{6}\right)}$ Institut Jordi Gol i \\ Gurina. Barcelona. Spain.
}

Introduction: Lifestyle modifications can be socially contagious. To date, it is unknown whether lifestyle treatments to correct excess weight extend their healthy effect to the rest of the family.

Objectives: To analyze the halo effect of anthropometric changes, dietary habits, adherence to the Mediterranean Diet (MedDiet), and physical activity (PA) on the family nucleus of obese/overweight patients receiving a lifestyle intervention.

Methods: This study was performed in the context of the PREDIMEDplus study (a randomized, parallel trial with an intensive lifestyle intervention (restrictive MedDiet $+\mathrm{PA}$ ) and a control group with MedDiet recommendations, in obese/overweight participants with metabolic syndrome) in a subsample of relatives of the study volunteers to determine whether promoting 
both interventions in PREDIMEDplus study subjects for 1 year modified the relatives' health status $(n=101)$. We also performed a cross-sectional analysis of a subsample of participants $(n=618)$ who were included in the study 1-2 years ago to analyze the halo effect changes between the two trial groups.

Relatives were asked to fill in several questionnaires of 1) anthropometric measurements; 2) adherence to the MedDiet. Differences in changes among the relatives of both intervention groups were assessed by multivariate mixed models.

Results: In the longitudinal sub-study, among the relatives of the participants randomized in the intervention group $(n=48)$, a borderline increase in MedDiet adherence was observed (mean $\pm \mathrm{SD}),(0.9 \pm 2.03 ; \mathrm{P}=0.056)$, and a non-significant decrease in weight. In contrast, no significant changes were observed in the control group $(\mathrm{n}=53)(\mathrm{P}>0.05)$.

In the cross-sectional analysis, a significantly greater MedDiet adherence was observed in relatives of the intervention group $(n=306),(8.85 \pm 2.20)$ compared to those of the control group $(\mathrm{n}=312)(8.35 \pm 2.11)(\mathrm{P}=0.004)$. No significant differences were observed in terms of weight.

Conclusions: An intensive intervention with a hypocaloric MedDiet and PA promotes an increase in the adherence to this dietary pattern in the relatives of the study volunteers.

Conflict of interest: Authors declare no conflict of interest.

Key words: halo effect / lifestyle intervention / obesity.

\section{6 \\ FRUIT AND VEGETABLE CONSUMPTION: NUTRI- TION AND HEALTH IN 21ST CENTURY SPAIN}

\author{
(1)P. Arroyo Uriarte; (1)E. Ruiz Moreno; (1)P. Rodriguez Alonso; ${ }^{(1)}$ T. \\ Valero Gaspar; ${ }^{(1)}$ J.M. Ávila Torres; ${ }^{(1,2)}$ G. Varela Moreiras. \\ (1)Spanish Nutrition Foundation (FEN). Madrid. Spain; ${ }^{(2)}$ CEU San \\ Pablo University. Madrid. Spain.
}

Introduction: The Spanish Nutrition Foundation (FEN) develops educational and promotional strategies for the consumption of fruit and vegetables, including its 2017 Strategic Plan and $16^{\text {th }}$ National Nutrition Day.

Objectives: To develop a report on the current situation of fruit and vegetable consumption in Spain and its benefits.

Methods: A study of the intake of fruit and vegetables was carried out using the data of the Household Food Consumption Panel, the ANIBES Study and different surveys such as ENUCAM and UNINUT. The properties, recommendations and influence on population health and the characteristics of a sustainable consumption were reviewed. Subjects such as allergies, food waste, myths and recipes were also addressed.

Results: According to the Panel, the consumption of vegetables has decreased by $40.4 \%$ since 1964, being $451 \mathrm{~g} /$ person/day that year and dropping to $269 \mathrm{~g} /$ person/day in 2015. Fruit consumption, however, increased significantly over the same period, although a short-term decrease was observed. The recommendation for each group is $>2-3$ servings/day, whereas 1.3 servings of veg- etables (without potatoes) and 1.5 servings of fruit are consumed per day in Spain. The ANIBES study estimates a consumption of fruits and vegetables of $345 \mathrm{~g} /$ person/day, the elderly (65-75 years) being the group with the highest intake and adolescents the lowest. In all the groups fruit and vegetables provided less than $5 \%$ of the daily energy, but they contributed $41 \%$ of fibre to the diet, $70 \%$ of vitamin C, $38 \%$ of vitamin $\mathrm{A}$ and $33 \%$ of folates.

Conclusions: It is necessary to promote the consumption of fruit and vegetables to comply with recommendations and improve the diet of the Spanish population.

Conflict of interest: Authors declare no conflict of interest. Key words: fruits / vegetables / servings.

\section{7 \\ TRENDS IN OBESITY DEVELOPMENT AND ITS RELATIONSHIP WITH SCREEN TIME IN CHIL- DREN FROM ARAGON. THE CALINA STUDY}

\author{
${ }^{(1)}$ M.L. Miguel-Berges; ${ }^{(1)}$ A. Santaliestra-Pasias; ${ }^{(1)}$ P. Flores-Barrantes; \\ ${ }^{(2)}$ I. Iglesia-Altaba; ${ }^{(1)}$ M. L. Álvarez; ${ }^{(1)}$ L. Moreno; ${ }^{(3)}$ G. Rodriguez. \\ ${ }^{(1)}$ Growth, Exercise, Nutrition and Development (GENUD) \\ Research Group. University of Zaragoza. Zaragoza. Spain; ${ }^{(2)}$ Red \\ de Salud Materno-infantil y del Desarrollo (SAMID). RETICS \\ financed by Instituto de Salud Carlos III (ISCIII) (RD16/0022); \\ ${ }^{(3)}$ Departamento de Pediatría, Radiología y Medicina Física. \\ Universidad de Zaragoza. Zaragoza. Spain.
}

Introduction: Sedentary time in front of a screen during childhood is increasing and has been associated with obesity development. However, there are indications of a reverse association in this relationship, with children who develop obesity becoming more sedentary.

Objectives: To analyse the longitudinal development of the $\mathrm{z}$-score Body Mass Index (zBMI) for age and its relationship with total screen time (ST) after seven years of follow-up.

Methodology: A cohort of 381 children (40.7\% boys) from the Growth and Feeding during Infancy and Early Childhood in Aragon (CALINA) longitudinal study, born in Zaragoza in 2009-2010, were included. Weight and height were measured at 0,6 and 12 months, and 3 and 7 years, calculating the zBMI for age according to Cole et al. The total ST was registered at 7 years, and categorized based on the recommendations ( $<2$ hour/day and $>2$ hour/day). An analysis of covariance was carried out to analyse the association between the evolution of the $\mathrm{z}$-BMI and the total $\mathrm{ST}$, adjusted by maternal education and gender.

Results: Only $2.8 \%$ of the sample was large at the gestational age. After 1, 3 and 7 years of follow up, the proportion of children with overweight $(4.4 \%, 12.4 \%$ and $14.2 \%$, respectively) and obesity $(0.8 \%, 2.3 \%$ and $6.5 \%$, respectively) increased. Those children who exceeded the total ST recommendations at seven years, comped to those who did not, had a higher zBMI at birth $(0.48 \pm 0.09$ versus $0.40 \pm 0.09)$, 3 years $(0.28 \pm 0.09$ versus $0.20 \pm 0.09)$ and 7 years $(0.67 \pm 0.09$ versus $0.60 \pm 0.09)$. 
Conclusions: zBMI increases longitudinally across age, and was associated with high total ST after 7 years of follow up.

Conflict of interest: Authors declare no conflict of interest.

Key words: screen time / obesity / obesity development.

\section{8 \\ FOOD CONSUMPTION IN SPANISH HOUSE- HOLDS COMPARED TO HEALTHY FOOD MAR- KET RECOMMENDATIONS}

\author{
(1)P. Rodríguez Alonso; (1)E. Ruiz Moreno; (1)T. Valero Gaspar; (1) J.M. \\ Ávila Torres; ${ }^{(1,2)} G$. Varela Moreiras. \\ (1)Spanish Nutrition Foundation (FEN). ${ }^{(2)}$ CEU San Pablo \\ University. Madrid, Spain.
}

Introduction: To analyze dietary patterns in Spanish households and evaluate the recommendation for food groups.

Objectives: To analyse the food consumption servings in Spanish households and to evaluate the adequacy of the servings recommended by the FEN Healthy Food Market.

Methods: Food consumption in Spanish households was studied; the data sample consisted of consumption and distribution data obtained from the Food Consumption Survey (FCS), in collaboration with the Spanish Nutrition Foundation (FEN). After knowing the grams per person per day of the food consumed, daily and weekly servings were calculated through the weights, measures and portion sizes of foods and beverages and compared with the FEN Healthy Food Market recommendations.

Results: In 2016 meat and meat product consumption (7.8 servings/week) was higher than the recommendation; on the contrary, the consumption of cereals and derivatives, and potatoes (3.6 servings/day), vegetables and greens (1.4 servings/day), fruit (1.5 servings/day) and legumes and pulses ( 0.9 servings/day) was lower than what is considered adequate. Groups that adjust most to recommendations were milk and derivatives ( 2.1 servings/day), fish (3.4 servings/week) and eggs (2.6 servings/week).

Conclusions: The results show an excessive consumption of meats and meat products and insufficient consumption of cereals and derivatives, vegetables and greens, fruit and legumes and pulses. It is necessary to design strategies that encourage a healthy diet that follows recommendations more closely.

Conflict of interest: Authors declare no conflict of interest.

Key words: servings / recommendations.

\section{9}

\section{ACCURACY OF BODY MASS INDEX CUTOFFS FOR CLASSIFYING OBESITY IN SPANISH CHILDREN}

\author{
${ }^{(1)}$ L. Ibáñez; ${ }^{(1)}$ I. Iglesia; ${ }^{(1)}$ M.L. Miguel-Berges; ${ }^{(1)}$ P. Flores-Barrantes; \\ ${ }^{(1)}$ M.L. Álvarez-Sauras; ${ }^{(2)}$ P. Samper; ${ }^{(1)}$ L. Moreno; ${ }^{(2)}$ G. Rodríguez- \\ Martínez. \\ ${ }^{(1)}$ Growth, Exercise, Nutrition and Development (GENUD) \\ Research Group. Universidad de Zaragoza. Spain; \\ (2) Departamento de Pediatría. Facultad de Medicina. Universidad \\ de Zaragoza. Spain.
}

Introduction: The prevalence of obesity has increased among children. For obesity diagnosis, different cutoff points are suggested. Thus, before selecting the cutoff, several aspects should be considered.

Objectives: To assess the ability of the IOTF and WHO BMI cutoffs to identify overweight-obesity in a sample of 7.5-year-old Spanish children, and to make a comparison with the values of fat mass percentage (\%FM) determined by Dual X-ray absorciometry (DXA).

Methods: The subjects studied were 420 children (222 boys) from the Growth and Feeding during Breastfeeding and Early Childhood in Aragoneses Children (CALINA) study. Height (SECA stadiometer) and weight (TANITA BC-418) were measured and the BMI was calculated. Two international references (IOFT and WHO) were used as cut-off points to define overweight or normal weight. The WHO system defines overweight as a BMI $>1 \mathrm{SD}$, corresponding to a 97.7 percentile from the WHO reference population. The IOTF cut-off is an extrapolation of the adult BMI cut-off points for overweight $\left(25 \mathrm{~kg} / \mathrm{m}^{2}\right)$.

Results: Differences in classification between normal weight and overweight children occurred when using IOFT and WHO cutoffs ( $\mathrm{p}<0.001$ ). 21 boys were classified as overweight/obese based on the WHO criteria, whereas the same cases were considered as normal weight by the IOTF standards. Using the WHO cutoff, 6 girls were classified as obese, but of normal weight according to IOTF. Additionally, a higher correlation was observed between the \%FM measured by the DXA and the Z-scores of IOTF than the WHO scores in the Spanish children $(r=0.67$ and 0.65 , respectively, and $\mathrm{p}$ values).

Conclusions: The prevalence of obesity classified by various cut-off points was variable. There was a high correlation between the BMI Z-score values of the WHO and IOTF and the \%FM. This therefore suggests that the selection of the cut-off points for obesity should be viewed with caution.

Conflict of interest: Authors declare no conflict of interest.

Key words: BMI / obesity / DXA / cut-off points. 


\section{0}

\section{BREAKFAST CONSUMPTION AND ITS RELA- TIONSHIP WITH THE DIET QUALITY INDEX IN EUROPEAN ADOLESCENTS: THE HELENA STUDY}

\author{
${ }^{(1)}$ N. Giménez-Legarre; ${ }^{(1)}$ A.M. Santaliestra-Pasías; ${ }^{(2)}$ A. Kafatos; ${ }^{(3)} Y$. \\ Manios; ${ }^{(4)} D$. Molnar; ${ }^{(5)}$ K. Widhalm, ${ }^{(1)}$ L.A. Moreno. \\ (1)GENUD (Growth, Exercise, Nutrition and Development) \\ Research Group. Facultad de Ciencias de la Salud. Universidad \\ de Zaragoza. Zaragoza. Spain; (2)University of Crete. School of \\ Medicine. Heraklion. Greece; ${ }^{(3)}$ Department of Nutrition and \\ Dietetics. School of Health Science and Education. Harokopio \\ University. Athens. Greece; ${ }^{\left({ }^{4}\right)}$ Department of Pediatrics. Medical \\ Faculty. University of Pécs. Pécs. Hungary; ${ }^{(5)}$ Department of \\ Pediatrics. Medical University of Vienna. Vienna. Austria.
}

Introduction: Breakfast consumption plays a protective role in preventing excess adiposity in children and adolescents, and has been associated with high diet quality intake.

Objectives: To describe the association between the habit of breakfast consumption and Diet Quality Index (DQI) in European adolescents from the HELENA study.

Methods: A subsample of adolescents from a multinational cross sectional study was included ( $n=1943,44.7 \%$ males; aged 12.5-17.5 years). The Food Choices and Preferences questionnaire was used to obtain breakfast consumption (consumers, occasional consumers and skippers), and two non-consecutive $24 \mathrm{~h}$ recalls were used to obtain the daily intake and to calculate the subsequent DQI and the DQI including the physical activity levels (DQI-PA) obtained using accelerometers. Analyses of covariance (ANCOVA) were used to compare breakfast consumption and DQI and DQI-PA, adjusting by education level of the mother, energy intake and body mass index (BMI).

Results: In both genders, significant differences were observed between the breakfast consumption categories. In males, breakfast consumers had high DQI scores (51.66, CI 95\%: 50.13, 53.18) compared with those who usually skipped breakfast (43.99, CI 95\%: 41.77, 46.21) $(\mathrm{p}<0.001)$. Female breakfast consumers also had a high DQI score $(56.78$, CI 95\%: 55.49, 58.06) compared with those who usually skipped breakfast (50.98, CI 95\%: 49.44, 52.52) $(\mathrm{p}<0.001)$. In boys, breakfast consumers also had high DQI+PA scores $(62.30$, CI 95\%: 61.03, 63.56) compared with those adolescents who skipped breakfast (55.32, CI 95\%: 53.46, 57.18) $(\mathrm{p}<0.001)$. Finally, in girls, breakfast consumers had high DQI+PA scores (65.81, CI 95\%: 64.72, 66.90) compared with adolescents who skipped breakfast (61.64, CI 95\%: 60.34, 62.94) ( $<<0.001)$.

Conclusions: In adolescents usual breakfast consumption was associated with a high DQI.

Conflict of interest: Authors declare no conflict of interest.

Key words: breakfast / diet quality index / adolescents.

\section{1}

\section{MATERNAL BODY MASS INDEX IS ASSOCIATED WITH BODY MASS INDEX OF 7-YEAR-OLD CHIL- DREN FROM ARAGON, SPAIN}

\author{
(1)P. Flores-Barrantes; (2)I. Iglesia Altaba; ${ }^{(1)}$ M.L. Miguel Berges; ${ }^{(1)}$ L. \\ Moreno Aznar; ${ }^{(3)}$ M.P. Samper Villagrasa; ${ }^{(4)}$ G. Rodríguez Martínez.
}

${ }^{(1)} G r o w t h$, Exercise, Nutrition and Developmet (GENUD) Research Group. Zaragoza. Spain; ${ }^{(2)}$ Instituto de Investigación Sanitaria Aragón (IIS Aragón). Zaragoza. Spain; ${ }^{(3)}$ Department of Pediatrics. Universidad de Zaragoza. Zaragoza, Spain; ${ }^{(4)}$ Hospital Clínico Universitario Lozano Blesa. Zaragoza. Spain.

Introduction: Maternal body mass index (BMI) before pregnancy is known to be a predictor of childhood overweight, but it remains unclear if both maternal and paternal BMI in later life have an influence on their children's overweight risk.

Objectives: To assess if maternal and/or paternal BMI influences the BMI of children at 7 years of age.

Methods: 369 children (53.1\% boys) of the Growth and Feeding during Early Childhood in Aragonese Children (CALINA) study and their parents were included. The BMI of parents was self-reported on the day of their child's evaluation at 7y. Childrens' weight was measured with a BC418MA Tanita ${ }^{\circledR}$ and weight status was estimated based on their BMI Z Score for age, according to the World Health Organization Growth Standards. Fat mass was measured by Dual X-Ray absortiometry. Linear regression adjusted by educational level was used to assess relationships between parental BMI and children's z-BMI Scores and Fat Mass Index (FMI).

Results: Maternal BMI had a positive correlation with children's $z$-BMI ( $\beta=0.114, p$ 0.047), whereas the BMI of fathers seems not to have had a significant influence $(B=0.016, \mathrm{p} 0.912)$. This effect was only seen in BMI and not in FMI (p 0.77 and p 0.895 for mothers and fathers, respectively).

Conclusions: Higher maternal BMI was associated with an increased z-BMI in children at the age of 7y. Parental BMI does not seem to be related to FMI.

Conflict of interest: Authors declare no conflict of interest.

Key words: childhood obesity / body mass index.

\section{2}

\section{NUTRITIONAL DATA (ENERGY AND CALORIC PROFILE) DECLARED IN MENU ROTATION OF PUBLIC SCHOOLS IN THE COMMUNITY OF MA- DRID (2007-2014)}

\footnotetext{
${ }^{(1)}$ T. Valero; ${ }^{(1)}$ E. Ruiz; ${ }^{(1)}$ P. Rodríguez; S. ${ }^{(2)}$ S. Del Pozo; ${ }^{(3)}$ A. García; ${ }^{(1)} J . M$. Ávila; $(1,3)$ G. Varela-Moreiras; ${ }^{(4)}$ C. Cuadrado.

${ }^{(1)}$ Fundación Española de la Nutrición (FEN). Madrid. Spain.

${ }^{(2)}$ Healthy Applications for you S.L. (HAPPY). Madrid. Spain;

${ }^{(3)}$ Departamento de Ciencias Farmacéuticas y de la Salud. Universidad CEU-San Pablo. Campus de Montepríncipe. Madrid. Spain. ${ }^{(4)}$ Departamento de Nutrición y Ciencias de los Alimentos. Universidad Complutense de Madrid. Madrid. Spain.
} 
Introduction: Madrid's School Meals Program evaluates catering companies in terms of compliance with specific regulations. It considers the menu preparation and programming and improvements in the provision of lunchroom service in public schools. The composition and variety of menu rotation and nutritional information were reviewed, together with the chemical analysis of duplicate portions of menus collected in the visits.

Objectives: To review the nutritional data declared in the menu rotation of public schools between 2007-2014 to then evaluate their trends throughout the academic years.

Methods: The nutritional data provided by the catering companies were checked considering the frequency in which the nutritional assessment of the menus was given (daily, weekly or monthly) and the units used (grams, \%, kcal, etc.). The energy content and the caloric profile from the menu rotation were reviewed according to the academic years.

Results: The declared mean average energy content of the menus served (749 kcal/d; 369-1380) was slightly higher than that recommended for the population studied (6-9 years). However, significant statistical differences were observed throughout the academic years ( $\mathrm{p}<0.0005)$, closing the gap between the stated values. The declared mean caloric profile showed a high contribution of energy from proteins (17.0 \% (8.7-36.1)) and a low contribution from carbohydrates $(45.8 \% ; 19.5-67.2)$, while the contribution of lipids was in the upper limit of the established range (35.0\%; 13.9 68.8). There were no statistical differences, however, when considering the trends over the academic years, when there was a better contribution to the total energy from the group of carbohydrates. Similarly, lipids decreased slightly in the last years.

Conclusions: There has been an improvement in the information provided in the school menu rotation thanks to the review carried out within the Madrid's School Meals Program.

Conflict of interest: Authors declare no conflict of interest.

Key words: schools / meals /child / food.

\section{3 \\ MODERATE AGED WINE CONSUMPTION INDUC- ES DISPLACEMENT OF UNHEALTHY FOODS IN THE USUAL DIET IN HIGH CASRDIOVASCULAR RISK MALES}

\author{
${ }^{(1)}$ I. Roth; ${ }^{(1)}$ M. Ribó-Coll; ${ }^{(1,2)}$ R. Casas; ${ }^{(1,2)}$ R. Estruch. \\ ${ }^{(1)}$ Department of Internal Medicine. Hospital Clínic. Institut \\ d'Investigació Biomèdica August Pi i Sunyer. Universitat de \\ Barcelona. Spain; ${ }^{(2)}$ CIBERobn Physiopathology of Obesity and \\ Nutrition. ISCIII. Madrid. Spain.
}

Introduction: Previous studies have suggested that moderate consumption of alcoholic beverages, wine in particular, has beneficial effects on the cardiovascular system. The possible protective mechanisms of alcoholic beverages may due to the alcohol component (ethanol), non-alcoholic products containing mainly polyphenols, or both. Wine consumption can also be associated with a high adherence to a traditional Mediterranean diet (MD)

XVII Conference of SEÑ and X Meeting of ACCA with reduced incidence of coronary heart disease and mortality. Although wine is energy-dense, there is no evidence that its regular consumption results in weight gain.

Objectives: To study the hypothesis that regular alcohol consumption (Andalusian white wine, AWW) would not induce weight gain by displacing other high-energy foods from the diet.

Methods: We embarked on a randomized crossover feeding trial with 40 male subjects at high cardiovascular risk. All subjects received $0.30 \mathrm{~g}$ of alcohol $/ \mathrm{kg} / \mathrm{day}$ in the form of $\mathrm{AWW}$ or gin. The subjects did not receive any recipes or advice to change the diet. Throughout the trial we assessed changes in dietary intake (3-day food records), physical activity and body weight.

Results: The trial was completed by $95 \%$ of participants. The mean consumption was $255 \mathrm{ml} \mathrm{AWW}$ and $92 \mathrm{ml}$ gin. At the end of the intervention, energy intake increased by a mean of $212 \mathrm{kcal}$ $(\mathrm{p}=0.002)$ in $\mathrm{AWW}$ and $242 \mathrm{kcal} / \mathrm{d}(\mathrm{p}=0.003)$ in gin consumers. However, body weight only changed by a mean of $0.28 \mathrm{~kg}$ in AWW vs. $0.29 \mathrm{~kg}$ in gin $(\mathrm{p}=0.248 ; \mathrm{p}=0.116)$. There were no statistically significant between-group differences in changes of physical activity. The AWW intervention significantly reduced consumption of fast food $(p=0.013)$ and high-fat fermented dairy products $(p=0.029)$ and increased pulses $(p=0.034)$ compared to gin. However, the consumption of vegetables increased $(\mathrm{p}=0.046)$ after gin intake. On the other hand, both interventions significantly reduced the consumption of sweet foods ( $\mathrm{p}=0.031 ; \mathrm{p}=0.040$, respectively).

Conclusions: The consumption of AWW does not lead to weight gain and induces the spontaneous displacement of unhealthy foods.

Conflict of interest: Authors declare no conflict of interest.

Key words: wine / cardiovascular / food replacement / healthy.

\section{4 \\ MEDITERRANEAN DIET AND CARDIODIABESI- TY: AN UPDATED REVIEW FOR CLINICAL PRAC- TICE}

\author{
${ }^{(1,2)}$ A. Bach-Faig; ${ }^{(3)} M$. Franquesa; ${ }^{(4)}$ G. Pujol-Busquets; ${ }^{(5)}$ E. García- \\ Fernandez; ${ }^{(5)}$ L. Rico; ${ }^{(5)}$ L. Shamirian-Pulido; ${ }^{(6)}$ J. Smith.
}

\begin{abstract}
(1)Department of Health Sciences. Universitat Oberta de Catalunya. Barcelona. Spain; ${ }^{(2)}$ Food and Nutrition Area. Barcelona Official College of Pharmacists (COFB). Barcelona. Spain; ${ }^{(3)}$ REMAR-IVECAT Group. Health Science Research Institute Germans Trias i Pujol. Can Ruti Campus. Badalona. Spain; ${ }^{(4)}$ Division of Physiology. Department of Human Biology. Faculty of Health Science. University of Cape Town. Cape Town. South Africa; ${ }^{(5)}$ Health Sciences Faculty. Universitat Oberta de Catalunya. Barcelona. Spain; ${ }^{(6)}$ Division of Exercise Science and Sports Medicine. Department of Human Biology. Faculty of Health Science. University of Cape Town. Cape Town. South Africa.
\end{abstract}

Introduction: Food plays a key role in the development and prevention of cardiodiabesity, nomenclature involving obesity, metabolic syndrome (MetS), cardiovascular disease (CV) and/or type 2 diabetes (T2DM). The Mediterranean Diet (MedDiet) has 
been postulated as one of the dietary patterns of choice for patients with these health conditions.

Objectives: To perform a search on recent evidence-based studies for a systematic review of the MedDiet and its role as a preventive and therapeutic tool for cardiodiabesity.

Methods: Five clinical questions (CQ) were created through the methodology PICO (Patient, Intervention, Comparison, Outcome) in order to gather scientific evidence. A systematic review was conducted to provide evidence to answer the CQ.

Results: From September 2013 to July 2016, 20 new articles in scientific and medical literature were collected to respond to the CQ. There is high evidence to support that adherence to the MedDiet has a role in primary and secondary prevention of CDVs as well as in improving the health of patients with overweight or obesity. There is high-moderate evidence that the MedDiet could prevent an increase in weight gain and waist circumference in non-obese individuals and also improve MetS and reduce its incidence. Finally, there is moderate evidence of primary and secondary prevention of T2DM with the MedDiet.

Conclusions: The MedDiet has a role in preventing obesity and MetS in healthy individuals or those at risk, in mortality risk reduction in overweight or obese individuals, and decreases the incidence of T2DM and CVD in healthy individuals and reduces the severity of symptoms in individuals which already have the disease. Scientific evidence seems to support that adherence to the MedDiet is a preventive and therapeutic tool for cardiodiabesity.

Conflict of interest: Authors declare no conflict of interest.

Key words: mediterranean diet / diabetis mellitus / cardiovascular diseases / metabolic syndrome / obesity.

\section{5}

CONSUMPTION OF AGED WINE HAS BENEFICIAL EFFECTS ON BLOOD PRESSURE AND PLASMA NITRIC OXIDE IN MEN WITH HIGH CARDIOVASCULAR RISK

${ }^{(1)}$ I. Roth; ${ }^{(1)}$ M. Ribó-Coll; ${ }^{(1,2)}$ R. Casas; ${ }^{(1,2)}$ R. Estruch.

(1)Department of Internal Medicine. Hospital Clínic. Institut $\mathrm{d}^{\prime}$ Investigació Biomèdica August Pi iSunyer. Universitat de Barcelona. Barcelona. Spain; ${ }^{(2)}$ CIBER CB06/03 Fisiopatología de la Obesidad.

Introduction: Systemic hypertension remains the largest attributable risk factor for mortality worldwide. An approach that has been explored in the treatment of hypertension is the delivery of potent vasodilator nitric oxide (NO). Moderate alcohol consumption has been associated with a reduction of cardiovascular disease (CVD).

Objectives: The aim of this study was to evaluate the effects of acute consumption of Andalusian aged wine (AAW) or gin on blood pressure (BP) and plasma NO in men with high cardiovascular risk.

Methods: A total of 38 high-risk male volunteers aged between 55-80 years received at random $30 \mathrm{~g}$ of ethanol a day in the form of AAW or gin for 3 weeks. The study was an open, randomized, controlled, crossover trial, to compare the effects of moderate consumption of AAW and gin after 3 weeks of intervention.

Results: The BP analysis after consumption of each alcohol beverage showed a significant decrease in systolic BP (SBP) and diastolic BP (DBP) for AAW $(\mathrm{P}<0.033 ; \mathrm{P}<0.030)$, and $\mathrm{NO}$ also increased $(P<0.013)$. No changes were observed after the gin intervention. When both interventions were compared, a significant decrease in SBP and DBP ( $\mathrm{P}=0.039$, both) was observed for AAW.

Conclusions: Moderate consumption of AAW could significantly increase NO bioavailability and reduce SBP and DBP. The numerous polyphenols contained in wine probably mediate the expressional upregulation of NO. It is reasonable to consider NO as a therapeutic target for CVD due to its antihypertensive effects.

Conflict of interest: Authors declare no conflict of interest.

Key words: nitric oxide / cardiovascular disease / wine.

046

\section{ASSOCIATION OF EARLY NUTRITION AND GEN- DER WITH METABOLIC RISK IN HEALTHY CHIL- DREN AT 4 YEARS OF AGE}

\author{
${ }^{(1,2)}$ C. Campoy; ${ }^{(1,2)}$ A. Nieto-Ruiz; $;^{(1,3)} N$. Sepúlveda-Valbuena; ${ }^{(1,2)} E$. \\ Diéguez; ${ }^{(1,2)}$ F. Herrmann; ${ }^{(4)}$ M.T. Miranda; ${ }^{(5)}$ R. De Castellar.
}

(1)EURISTIKOS Excellence Centre for Paediatric Research. University of Granada. Spain; ${ }^{(2)}$ Departmentof Paediatrics. School of Medicine. University of Granada. Granada. Spain; ${ }^{(3)}$ Nutrition and Biochemistry Department. Faculty of Sciences. Pontificia Universidad Javeriana. Bogotá. Colombia; ${ }^{(4)}$ Department of Biostatistics. School of Medicine. University of Granada. Spain; ${ }^{(5)}$ Medical Department of Ordesa Laboratories, S.L. Barcelona. Spain.

Introduction: During infancy it is possible to detect numerous risk factors that determine long-term health status. Excess weight in the first years of life increases the risk of metabolic syndrome. Biological factors, such as gender, may determine greater predisposition to develop these diseases.

Objectives: To analyze the relationship between a new infant formula and gender with metabolic risk in healthy children at 4 years of age.

Methods: A total of 170 healthy infants between $0-2$ months of age were included in a randomized double-blind study to receive either a standard infant formula $(\mathrm{F} 1: \mathrm{n}=85)$ or one supplemented with different compositions of long-chain polyunsaturated fatty acids (LC-PUFAs), milk fat globule membrane (MFGM), synbiotics, sialic acid, nucleotides and gangliosides (Nutriexpert ${ }^{\circledR}$ factor) $(\mathrm{F} 2: \mathrm{n}=85)$. As a control group, 50 breastfed infants $(\mathrm{BF})$ were included. Anthropometric measures were evaluated at 4 years of age. The sum of skinfolds (biceps, triceps, subscapular and suprailiac) and Slaughter's equations was used to calculate body fat mass (BFM). Additionally, blood pressure (BP) was measured. In the statistical analysis normal distribution was assumed using the Kolmogorov-Smirnov test; ANOVA, Student t-test and Chi-Square test and SPSS 22.0 were performed. 
Results: At 4 years of age, 102 children were assessed (F1: $\mathrm{n}=46 / \mathrm{F} 2$ : $\mathrm{n}=43 / \mathrm{BF}=13$ ) (Boys $\mathrm{n}=60 /$ Girls $\mathrm{n}=42$ ). There were no differences between study groups in BFM and BP. However, girls presented higher BFM and were classified as overweight and obese more frequently than boys $(\mathrm{p}<0.001)$. Analysis revealed that girls presented a normal-high systolic BP compared to boys $(\mathrm{p}=0.002)$.

Conclusions: Metabolic risk factors are associated with gender and could determine the development of metabolic pathologies later in life. At 4 years of age, excess fat mass and high blood pressure are associated with the female gender. An adequate nutrition during the first months of life might reduce the risk of developing metabolic disorders.

Conflict of interest: Funded by Ordesa Laboratories.

Key words: metabolic risk / early nutrition.

\section{7 \\ EATING HABITS AND PHYSICAL ACTIVITY IN CHILDREN FROM A SCHOOL IN ANTEQUERA AC- CORDING TO GENDER}

(1)J. García; (1)V. Torres; ${ }^{(1)}$ L. Macías; ${ }^{(2)}$ R. Marfil; (1)J.A. Sánchez.

\author{
(1)Unidad de Protección de la Salud. Área Sanitaria norte \\ de Málaga. Antequera. Spain; ${ }^{(2)}$ Distrito Sanitario Granada \\ Metropolitano. Granada. Spain.
}

Introduction: Obesity has its origin in the interaction of genetic and environmental factors such as dietary habits, physical activity and the degree of sedentary lifestyle. Identifying differences in eating habits and physical activity among school-age children is important in the design of intervention activities.

Objectives: To identify differences in eating habits and physical activity among children of a school of secondary education in Antequera for the design of intervention activities.

Methods: A descriptive cross-sectional study was carried out on a population of 54 children of 14 and 15 years of age. The main variables were the eating habits and physical activity. The collection and analysis of data was carried out with the program R.2.10.

Frequencies and percentages were used in the description of qualitative variables. To determine the existence of statistically significant differences according to gender the Chi-square test was performed. The results were considered significant when they presented a $\mathrm{p}<0.05$.

Results: $33.6 \%$ of students had a high nutritional level, while the remaining $66.4 \%$ had a medium and low nutritional level. The physical activity of $24.6 \%$ of schoolchildren was adequate, while $75.4 \%$ had low levels. There was no significant relationship between the nutritional level and physical activity.

Conclusions: A large number of school children should improve their lifestyle. The results obtained provide a starting point for establishing guidelines and activities for an intervention program for the prevention of obesity based on modification of eating habits and increasing physical activity.

Conflict of interest: Authors declare no conflict of interest.

Key words: obesity / dietary habits / activity.
048

RETINOL-BINDING PROTEIN-4 LEVELS AND LIVER FAT CONTENT IN SUBJECTS WITH NON-ALCOHOLIC FATTY LIVER DISEASE AND OBESITY

\author{
${ }^{(1)}$ I. Cantero; ${ }^{(1)}$ I. Abete; A. ${ }^{(1)}$ A. Marin-Alejandre; ${ }^{(2)} J . I$. Monreal; ${ }^{(2)} M$. \\ Elorz; ${ }^{(2)}$ J.I. Herrero; ${ }^{(1)}$ J.A. Martinez; ${ }^{(1)}$ M.A. Zulet. \\ (1)Ciencias de la Alimentación Fisiología y Salud. Universidad \\ de Navarra. Pamplona. Spain; ${ }^{(2)}$ Clínica Universidad de Navarra. \\ Pamplona. Spain.
}

Introduction: RBP-4 has been described as a potential hepatokine linking non-alcoholic fatty liver disease (NAFLD) and obesity co-morbidities.

Objectives: The aim of this study was to evaluate the relationships between RBP-4 and hepatic fat content measured by Magnetic Resonance Imaging (MRI) in obese people with NAFLD.

Methods: A total of one hundred and nineteen overweight or obese adult patients with NAFLD, assessed by abdominal ultrasonography (Siemens ACUSON S2000 and S3000) and Magnetic Resonance Imaging (MRI, Siemens 1,5 T), were included in this cross-sectional study. Participants with $\geq 5.5 \%$ of hepatic fat were considered as the cut off category for higher lipid content. RBP-4 plasma levels were measured by specific kits using an automated analyzer system (Triturus, Grifols). Additionally, body composition and biochemical measurements were assessed. This study was registered as follows: FLiO: Fatty Liver in Obesity study; NCT03183193.

Results: The median of the percentage of hepatic fat content was 6.4+ (3.8-10.9) in the recruited population (overweight/obese with NAFLD). Waist circumference, glucose, HDL-c, triglycerides, HOMA-IR and HOMA- $\beta$ as well as RBP-4 showed significantly worse results in those patients with a higher hepatic fat content $(\mathrm{p}<0.05)$. Thus, variables associated with the liver fat content were: RBP-4 $(\beta=0.2 ; \mathrm{R} 2=6.9 ; \mathrm{p}<0.002)$ and HOMA $-\beta(\beta=0.6 ; \mathrm{R} 2=5.4$; $\mathrm{p}<0.006)$. When these variables were jointly considered together with android fat mass, the predictors of the model explained up to $12.6 \%$ of the variation of the hepatic fat content (adjusted-R2= $0.126 ; \mathrm{p}<0.001$ ).

Conclusions: In addition to body composition and metabolic status, these findings suggest that RBP-4 should also be considered in liver management and to understand the involved pathological mechanisms.

Conflict of interest: Authors declare no conflict of interest.

Key words: obesity / RBP-4 / NAFLD / liver.

Ann Nutr Metab 2018;73 (suppl 2): 1-93 DOI: $10.1159 / 000490752$ 


\section{9}

ADHERENCE TO THE MEDITERRANEAN DIET AND NON-ALCOHOLIC FATTY LIVER DISEASE AMONG MEN AND WOMEN WITH METABOLIC SYNDROME FEATURES

\author{
${ }^{(1)} V$. Bullón Vela; ${ }^{(1,2)}$ I. Abete; ${ }^{(1,2)}$ M. Angeles Zulet; ${ }^{(1,2)} \mathrm{J}$. Alfredo \\ Martínez. \\ (1)Departamento de Nutrición, Ciencias de la Alimentación y \\ Fisiología. Centro de Investigación en Nutrición. Pamplona. \\ Spain; ${ }^{(2)} \mathrm{CIBER}$ obn Physiopathology of Obesity and Nutrition. \\ Instituto de Salud Carlos III. Madrid. Spain.
}

Introduction: Non-alcoholic fatty liver disease (NAFLD) is the most common chronic hepatic disorder in subjects with metabolic syndrome diagnosis. Emerging data suggest that Mediterranean Dietary patterns (MedDiet) have been associated with healthy effects in subjects with NAFLD.

Objectives: To evaluate adherence to the Mediterranean Dietary pattern and the presence of NAFLD by means of non-invasive liver scores and biochemical-related variables in metabolic syndrome subjects stratified by sex.

Methods: A cross-sectional study encompassing 331 participants with metabolic syndrome was carried out. Patients were categorized according to sex: men $(n=181)$ and women $(n=150)$. Baseline anthropometric, biochemical and non-invasive hepatic indexes were assessed. Additionally, in order to evaluate the impact of the level of adherence to the MedDiet, data were analysed by a score designed by Trichopoulou et al. (2003).

Results: The adherence to a MedDiet pattern had a positive effect on body weight and BMI $(\mathrm{p}<0.05)$ in women. Men with greater adherence to the MedDiet reported significantly higher physical activity $(\mathrm{p}<0.05)$, whereas no differences were found concerning weight and BMI measurements. The nutritional analysis revealed that men with better MedDiet adherence had higher energy, carbohydrate and protein intake $(\mathrm{p}<0.001)$. A significant increase of fiber consumption was obtained in the highest MedDiet score in both sexes. A two-way ANOVA analysis revealed that gender not only exerts an influence on body composition but also on liver markers.

Conclusions: Our findings support that in addition to following a dietary pattern, gender might have clinical implications in liver status in subjects with metabolic syndrome.

Conflict of interest: Authors declare no conflict of interest.

Key words: NAFLD / metabolic syndrome / Mediterranean diet.

\section{0 \\ DO COMPOSITIONAL VALUES OF FOOD TABLES CORRESPOND WITH THOSE IN LABELS OF COM- MERCIAL MEAT PRODUCTS?}

\author{
S. Sánchez-Pérez; O. Comas-Basté; S. Hernández-Macias; N.C. \\ Muñoz-Esparza; M.L. Latorre-Moratalla; M.T. Veciana-Nogués; M.C. \\ Vidal-Carou.
}

\begin{abstract}
Departament de Nutrició, Ciències de l'Alimentació i Gastronomia. Facultat de Farmàcia i Ciències de l'Alimentació. INSA.UB. XaRTA. Universitat de Barcelona. Santa Coloma de Gramenet. Spain.
\end{abstract}

Introduction: The nutritional composition of some foods can be obtained from food composition tables and also by the mandatory nutrition information on the labels of commercial products. Food composition tables are extensively used in nutrition studies but there is some concern about the correspondence between their data and those reflected in the labels, which are perhaps more up-to-date. Meat products could be affected by this lack of correspondence.

Objectives: To compare the nutritional composition obtained from labels of commercial meat products with the BEDCA database ${ }^{1}$.

Methods: A total of 219 commercial meat products from the categories of dry-fermented sausages, and cured and cooked ham were revised, attending to their contents in proteins, fats, saturated fats and sodium. In addition, values posted in six different food composition tables ${ }^{1-6}$ were compared, including those of iron.

Results: Great variability in nutritional components of each of the commercial meat products was found. On average, contents did not agree with those of BEDCA, especially for dry-fermented sausages. In general, the content of fat and salt found on labels of dry-fermented sausages was higher than in the BEDCA database, whereas for cured ham it was the contrary. Cooked ham, which contains less fat, saturated fat and salt, showed the lowest variability and was the most accurately reflected in the tables. The iron content (not in labels) of all meat products was quite similar among the food composition tables.

Conclusions: The correspondence between the nutritional composition of meat products in the food composition database and labels of commercial meat products is low. This is a possible weak point in nutritional studies, which should be more critical with the reformulation of processed foods than is currently being proposed. The fat and salt of dry-fermented sausages are usually underestimated in the food composition database.

References: ${ }^{1}$ BEDCA (2006); ${ }^{2}$ Martín-Peña (1991); ${ }^{3}$ MataixVerdú et al. (2011); ${ }^{4}$ Moreiras et al. (2016); ${ }^{5}$ Ortega et al. (2004); ${ }^{6}$ Palma et al. (2008).

Conflict of interest: Authors declare no conflict of interest.

Key words: meat products / nutritional composition / food composition tables. 


\section{1}

\section{THE INTESTINAL MICROBIOTA COMPOSITION IS INFLUENCED BY CIRCADIAN RHYTHMS IN 344 FISCHER RATS.}

\author{
${ }^{(1)}$ A. Gual-Grau; (2) N. Boqué; ${ }^{(1)}$ C. Bladé; ${ }^{(1)}$ L. Arola

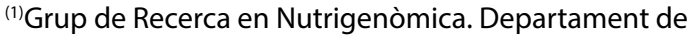 \\ Bioquímica i Biotecnologia. Universitat Rovira i Virgili. Tarragona. \\ Spain; (2)Unitat de Nutrició i Salut. Eurecat. Centre Tecnològic de \\ Catalunya. Reus. Spain.
}

Introduction: Gut microbiome composition is influenced by several environmental factors, while diet has been considered the major contributor to microbial changes in both humans and animals. Over the last years, host-microbiota co-metabolism has attracted attention due to its relevance in maintaining overall homeostasis. Moreover, circadian rhythms exert a key role in the regulation of several physiological processes, ultimately determining the health status. Thus, the intestinal microbiome has been considered a crucial element for the maintenance of circadian rhythms in the host, suggesting that microbiome rhythmicity is closely associated with host circadian clock gene expression.

Objectives: To evaluate the daily gut microbial fluctuations in healthy standard chow-fed rats.

Methods: Sixteen male Fischer 344 rats were fed with a standard diet for a 10-week period and maintained on a 12:12 light-dark cycle. Eight rats per group were sacrificed during diurnal (ZT0; 11 AM) or nocturnal (ZT12; $11 \mathrm{PM})$ conditions, respectively, and cecum was collected for metagenomic analysis.

Results: Our results indicated diminished proportions of the Lentisphareae phylum in the ZT12 group. At the genus level, principal component analysis performed from overall relative abundances revealed well-differentiated clusters according to ZT. Thus, up to 12 genera including Bifidobacterium and Clostridium showed significantly altered proportions between both groups. Remarkably, relative amounts of Lactobacillus were higher in the ZT0 group when compared to ZT12. The observed oscillation of Lactobacillus and other genera could reflect the effect of food consumption, which predominantly occurs during the night-time.

Conclusions: Our work identified specific daily gut microbiome variations defining microbial rhythmic patterns in standard chow-fed Fischer rats. These results emphasize the importance of considering daily microbial variations for nutritional studies focused on gut microbiome analysis.

Conflict of interest: Authors declare no conflict of interest.

Key words: microbiota / circadian rhythms / rhythmicity / lactobacillus.

\section{2 \\ THE AMED CERTIFICATION PROMOTES A HEALTHY DIET OUTSIDE HOME IN CATALONIA}

G. Miranda; G. Salvador; C. Castell; C. Cabezas; J. Guix.

Public Health Agency of Catalonia. Barcelona. Spain.
Introduction: The Mediterranean Diet is well known for being one of the healthiest diets. Since $31 \%$ of the Catalan population eats out at least once a week (Monday to Friday), in 2007 the Catalan Public Health Agency designed the Amed Certification to identify and certify Mediterranean Diet-promoting food establishments, whilst improving their offer. This pioneering project in Spain is addressed to restaurants, food chains and diners, especially in work surroundings.

Objectives: To improve and maintain over time the offer of selected typical Mediterranean foods before and after the certification.

Methods: Food offer was assessed by an online questionnaire through the Amed website. The data was then confirmed by a certified Dietitian-Nutritionist. Following the Amed criteria, a descriptive analysis was applied to obtain the improvement percentage of the food offer among the applicants (olive oil, vegetables and pulses, whole grain products, fresh fruit and low fat dairy desserts).

Results: The improvements in the food offer consisted of a $59 \%$ increase in the use of high-oleic-acid oil for frying, $22 \%$ in whole grain products (bread, pasta and rice), $28 \%$ in olive oil or high-oleic-acid oil for cooking, $14 \%$ in low fat dairy products for desserts, $5 \%$ in more vegetables and pulses in the first course, $7 \%$ in fresh fruit for desserts and $2 \%$ in lean meat and fish in the second courses. Olive oil was already being used as the main salad dressing by all applicants. There are currently 622 certified establishments in 144 municipalities, reaching more than 94,000 people per day.

Conclusions: The Amed certification guarantees food improvements within certified establishments, mainly concerning the change of common plant oil for high-oleic-acid oil for cooking and/ or frying. It also increases the offer of whole grain products, mainly bread, and fresh fruit and low fat dairy products for desserts.

Conflict of interest: Authors declare no conflict of interest.

\section{3}

\section{THE SCHOOL MEAL PROGRAM REVIEW}

\author{
M. Blanquer; G. Salvador; C. Castell. \\ Agència de Salut Pública de Catalunya (ASPCAT). Barcelona. \\ Spain.
}

Introduction: The School Meal Program Review (PReME), part of the PAAS strategy (Integral Plan of Health Promotion through Physical Activity and Healthy Eating) of the Public Health Agency of Catalonia (ASPCAT) is one of the activities of the 2.2 Health Plan Project 2011-2015. It 2006 in collaboration with the Department of Education it began offering the review of menu planning in schools in Catalonia.

Objectives: To improve the quality of menu planning in schools, and assess the acceptance of the meals served in order to increase the degree of satisfaction of consumers and initiate strategies that lead to reduced food waste.

Methods: Since 20064208 menu plans have been evaluated (phase 1), 1397 follow-up reports have been made on the imple- 
mentation and acceptance of suggestions for improvement (phase 2 ), and four pilot tests of sensory evaluation of food have been carried out (phase 3). After the request for a review through the territorial public health teams, the program is evaluated and the report is sent to the school. After 2-3 months, a new plan is requested along with a valuation questionnaire. A new report is made, comparing the evolution of the items evaluated.

Results: $87 \%$ considered it a useful tool and $82 \%$ believed that the report will lead to the improvement of menu planning. From 2006 to 2016, the following trends were observed in compliance with the recommendations: salads $(47 \% \cdot 59 \%)$, fresh fruits $3-4$ times/week $(86 \% \cdot 90 \%)$, fresh food $(57 \% \cdot 95 \%)$, vegetables $(73 \%$ -95.4\%) and olive oil or high oleic sunflower oil for cooking (34\% - $58 \%)$. In the sensory evaluation, the overall average rating of the dining room was 6.58 , from a sample of 582 children from 38 schools.

Conclusions: The PReME has a wide scope, is known by different stakeholders, is highly regarded and improves key aspects of menu planning.

Conflict of interest: Authors declare no conflict of interest.

Key words: school / lunch / meals. 


\section{Nutritions of Metabolism}

\author{
Reviewers: \\ Concepción Aguilera García \\ Universidad de Granada \\ Cristina Andrés Lacueva \\ Universitat de Barcelona \\ Diego Haro Bautista \\ Universitat de Barcelona \\ Pedro Marrero Gonzalez \\ Universitat de Barcelona \\ Alfredo Martínez Hernández \\ Universidad de Pamplona
}

\section{Oral Abstract Presentations}

\section{4}

\section{EFFECTS OF ALCOHOL AND THE NON-ALCO- HOLIC FRACTION OF BEER ON ATHEROSCLE- ROTIC BIOMARKERS: A RE-ANALYSIS BASED ON METABOLOMICS}

\author{
(1)P. Quifer; ${ }^{(2)}$ G. Chiva; ${ }^{(3)}$ R. Estruch; ${ }^{(1)}$ R.M. Lamuela-Raventós. \\ (1)Departamento de Nutrición, Ciencias de la Alimentación \\ y Gastronomia. Facultad de Farmacia y Ciencias de la \\ Alimentación. Universidad de Barcelona. Barcelona. Spain; \\ ${ }^{(2)}$ Centro de Investigación Cardiovascular (CSIC-ICCC). Barcelona. \\ Spain; ${ }^{(3)}$ Departamento de Medicina Interna. Hospital Clinic. \\ Instituto de Investigación Biomédica Auguist Pi i Sunyer \\ (IDIBAPS). Barcelona. Spain.
}

Introduction: Moderate alcohol consumption has been inversely associated with incidence of cardiovascular risk factors and all-cause mortality in several studies, independently of the type of alcoholic beverage consumed. However, the results of some studies indicate that fermented alcoholic beverages, like wine or beer, may provide additional protective effects due to their polyphenolic content.

Objectives: The aim of this work was to correlate the metabolomics data with cardiovascular risk factors such as the expression of several adhesion molecules in peripheral blood mononuclear cells, representative biomarkers of inflammation and biomarkers of endothelial injury.

KARGER XVII Conference of SEÑ and X Meeting of ACCA
Methods: In a crossover trial, 33 men at high cardiovascular risk were randomized to receive beer ( $30 \mathrm{~g}$ alcohol/d), the equivalent amount of polyphenols in the form of non-alcoholic beer, or gin (30 g alcohol/d) for 4 weeks. The urinary metabolome was analyzed by LC-HRMS (LC-Orbitrap). Biomarkers of endothelial injury and inflammation were quantified by Human Multi Analyte Profiling and molecules of adhesion were determined by immunofluorescence. Data was analyzed by performing a correlation matrix and constructing adjusted multilinear models.

Results: 16 urine metabolites were inversely correlated with the expression of adhesion molecules, inflammatory biomarkers, endothelial injury biomarkers and angiogenic factors. Moreover, six urinary metabolites were positively correlated with inflammatory biomarkers, endothelial injury biomarkers and angiogenic factors. Some polyphenols and beer compounds, such as co-humulone were correlated with inflammatory biomarkers and adhesion molecules.

Conclusions: Urinary metabolites after a regular and moderate intake of beer and nonalcoholic beer were correlated with the expression of adhesion molecules, inflammatory biomarkers, endothelial injury biomarkers and angiogenic factors.

Conflict of interest: Dr. Lamuela-Raventos and Dr. Estruch report receiving lecture fees from Cerveceros de España. Dr. Estruch reports serving on the boards of the Beer and Health Foundation and the European Foundation for Alcohol Research. These foundations were not involved in the study design, analysis and interpretation of data.

Key words: alcohol / polyphenols / metabolomics / cardiovascular diseases.

\section{5 \\ EFFECTS OF MAQUI EXTRACT (ARISTOTELIA CHILENSIS) ON WEIGHT CONTROL AND THE METABOLIC PROFILE OF MICE FED WITH A HIGH FAT DIET. \\ ${ }^{(1,2)}$ V. Sandoval; ${ }^{(1)}$ A. Femenias; ${ }^{(1,2)}$ U. Martinez-Garza; ${ }^{(1)}$ H. Sanz; \\ ${ }^{(1,2,3)}$ P.F. Marrero; ${ }^{(1,2,3)}$ D. Haro; ${ }^{(1,2)}$ J. Relat. \\ (1)Department of Nutrition, Food Sciences and Gastronomy. Faculty of Pharmacy and Food Sciences. Food and Nutrition Torribera Campus. University of Barcelona. Barcelona. Spain; (2)Institute of Research Nutrition and Food Security of the University of Barcelona (INSA.UB). Barcelona. Spain; ${ }^{(3)}$ Biomedical Institute of the University of Barcelona (IBUB). Barcelona. Spain.}

Introduction: Obesity is associated with important metabolic alterations such as insulin resistance, which is a prediabetic con- 
dition that appears early during the development of high-fat diet (HFD)-induced obesity. The fruit of Aristotelia chilensis, commonly known as maquis, is an anthocyanin-enriched berry. Different anthocyanin-rich formulations have shown anti-diabetic properties and different mechanisms have been proposed as at least partially responsible for this effect.

Objectives: To study the effect of a lyophilized maqui extract in the development of diet-induced obesity (DIO).

Methods: C57BL6 / J four-week-old male mice were fed for 16 weeks with a HFD and maqui extract added to the beverage water. Weight and food and drink intakes were recorded every two days. The glucose metabolism was evaluated by tests of glucose tolerance (GTT) and insulin tolerance (ITT), and gene expression analysis (qPCR) was measured in subcutaneous white adipose tissue (scWAT) and liver to define changes in the metabolic profile of the animals supplemented with respect to the controls.

Results: Our data show that maqui supplementation reduced the weight gain caused by a HFD, even with higher food intake. GTT and ITT showed an improvement in the glucose response and a higher sensitivity to insulin in the treated mice. Changes in the expression of genes were observed related to glucose/lipid metabolism in liver and thermogenic effects in scWAT.

Conclusions: A maqui extract improves the metabolism of glucose and decreases lipogenesis in the liver. It is able to control body weight in treated mice, even with higher food intake due to increased browning in scWAT.

Conflict of interest: Authors declare no conflict of interest.

Acknowledgments: SAF2013-41093 Ministry of Economy and Competitiveness, 2014SGR916 Generalitat de Catalunya, Ajut Associació Catalana de Diabetis a la Recerca en diabetes 2017, National Commission of Scientific and Technological Research CONICYT-Chile, National Council of Science and Technology CONACYT-Mexico.

Keys words: polyphenols / HFD / weight control / glucidic and lipid metabolism.

\section{6 \\ FOOD CONSUMPTION AND COMPOSITION OF GUT MICROBIOTA IN YOUNG MEXICAN STU- DENTS}

\author{
${ }^{(1)}$ A. Rodríguez; ${ }^{(2)} Z$. Reyes; ${ }^{(2)}$ U. López; ${ }^{(2)} A$. Vázquez; ${ }^{(1)}$ O. Acevedo; ${ }^{(1)} J$. \\ Rodríguez; ${ }^{(1)}$ C. Gómez; ${ }^{(2)}$ C. Beltrán: \\ ${ }^{(1)}$ Instituto de Nutrición y Tecnología de los Alimentos. Centro \\ de Investigación Biomédica. Universidad de Granada. Granada. \\ Spain; ${ }^{(2)}$ Centro de Investigaciones en Comportamiento \\ Alimentario y Nutrición. Centro Universitario del Sur. Universidad \\ de Guadalajara. México.
}

Introduction: Lifestyles, genetics and gut microbiota are key factors that influence the balance between energy expenditure and food consumption, which affects body weight and may lead to the development of obesity. Recent studies show that the gut microbiota is highly influenced by age, food consumption, genetic background and other factors.
Objectives: To analyze food consumption and its relationship with gut microbiota and body weight in young Mexican subjects

Methods: 50 participants (18-25 years) were included in the study and stratified as follows according to BMI: normal-weight $(n=15)$; overweight $(n=13)$ and obese $(n=12)$. Anthropometric and body composition parameters were determined in all subjects. A food frequency questionnaire (FFQ) was applied to obtain total energy, macronutrient and food group consumption. Bacterial DNA was extracted from fecal samples and the expression of bacterial phyla (Firmicutes, Bacteroidetes, Actinobacteria and Verrucomicrobia) was performed by quantitative real-time polymerase chain reaction (qPCR).

Results: Both groups showed a higher consumption than the recommended daily intake (RDI) for sugar, dairy, grains and cereals, vegetables and fruits. Most of the participants (from normal weight to obese) had a low consumption of protein and fiber. The expression of the bacteria Clostridium cocoides and Clostridium leptum was higher in the overweight/obese than normal-weight group ( $\mathrm{p}=0.017)$. Concerning the relationship between food consumption and the composition of the gut microbiota, we found a positive correlation between the Bacteroidetes group with fiber and fermented milk intake ( $\mathrm{p}=0.030$ and $\mathrm{p}=0.009$, respectively).

Conclusions: The results of this study indicate that food consumption in normal-weight and overweight/obese college students was similar, and both groups showed inadequate consumption of certain food groups, particularly, sugar, fiber and proteins. There is a relationship between gut microbiota, body weight and food consumption.

Conflict of interest: Authors declare no conflict of interest.

Key words: food consumption / gut microbiota.

\section{7 \\ VALIDATION OF CARNITINE PALMITOYLTRANS- FERASE 1 A AS A TARGET OF MIR-140 IN AML12 HEPATOCYTES}

\begin{abstract}
${ }^{(1,2)}$ A. Leniz; ${ }^{(2,3)}$ A. Fernández-Quintela; ${ }^{(2,3)}$ L. Aguirre; ${ }^{(2,3)}$ S. GómezZorita; ${ }^{(2)}$ N. Romo; ${ }^{(2)}$ I. Zarrabeitia; ${ }^{(2,3)}$ M.P. Portillo.

${ }^{(1)}$ Araba Integrated Health Care Organization. Basque Health Service (Osakidetza); (2)Nutrition and Obesity Group. Department of Nutrition and Food Science, University of the Basque Country (UPV/EHU) and Lucio Lascaray Research Institute. Vitoria. Spain; ${ }^{(3)}$ CIBEROBN. Instituto de Carlos III. Madrid. Spain.
\end{abstract}

Introduction: Micro RNAs (miRNAs), small non-coding RNAs, bind to the untranslated region of their target mRNAs. It has been described that miRNAs could regulate the pathogenesis of several liver diseases, including liver steatosis, which is the most common chronic liver disease worldwide. Carnitine palmitoyltransferase 1a (CPT1a), peroxisome proliferator-activated receptor $\alpha$ (PPAR $\alpha)$, diglyceride acyltransferase 2 (DGAT2) and mitochondrial transcription factor (TFAM) are involved in the development of liver steatosis. However, in some cases, the targets of the miRNAs are only predicted by mathematical algorithms and the regulation process has not been validated yet.

XVII Conference of SEÑ and X Meeting of ACCA 
Objectives: To identify whether some of the miRNAs theoretically described as regulators of CPT1a, PPARa, DGAT2 and TFAM proteins do in fact regulate these proteins through a target validation process.

Methods: miR-33, miR-19b, miR-140 and miR-93 were identified as potential regulators of CPT1a, PPARa, DGAT2 and TFAM by bioinformatics tools (MirBase, MiRecords). The selected miRNAs were transfected in AML12 hepatocytes using lipofectamine. The process was confirmed by the transfection of a fluorophore and the measurement of microRNA expression in the cultured cells by RT-PCR. Subsequently, protein and gene expressions of CPT1a, PPARa, DGAT2 and TFAM were assessed by immunoblot assay and RT-PCR, respectively.

Results: The AML12 hepatocytes transfected with miR-140 showed statistically lower protein expression of CPT1a, but not mRNA expression. DGAT2, another predicted target gene of miR140 , remained unchanged. The overexpression of miR-33, miR$19 \mathrm{~b}$ and miR-93 did not produce significant changes in gene and protein expressions of their predicted target genes.

Conclusions: The obtained results suggest that CPT1a is a real target of miR-140, showing that this miRNA inhibits the protein translation, but without affecting gene transcription.

Conflict of interest: Authors declare no conflict of interest.

Key words: microRNA / CPT1A / steatosis / AML12 / validation.

\section{8 \\ LIPID PROFILE IN SERUM OF ALZHEIMER'S DIS- EASE PATIENTS AND RELATIONSHIP WITH MFS- D2A FATTY ACID CARRIER}

\author{
${ }^{(1)} M . J$. Ruiz; ${ }^{(1)}$ M. Sánzhez-Campillo; ${ }^{(1)}$ A. Gázquez; ${ }^{(2)}$ A.J. Ruiz; ${ }^{(1)} E$. \\ Larqué; ${ }^{(3)}$ C. Antunez; ${ }^{(3)}$ J. Marín; ${ }^{(3)}$ F. Noguera. \\ (1)Physiology. University of Murcia. Murcia. Spain; (2)Biochemistry, \\ Molecular Biology B and Immunology. University of Murcia. \\ Murcia. Spain; ${ }^{(3)}$ Hospital Clínico Virgen de la Arrixaca. Murcia. \\ Spain.
}

Introduction: Patients with Alzheimer's disease (AD) have a brain deficiency of docosahexaenoic acid (DHA), which is important for cognitive function. However, little is known about its transfer across the blood-brain barrier in adulthood. In fact, DHA supplementation hardly increases DHA in cerebrospinal fluid, while omega-3 supplementation improves the levels of other $n-3$ fatty acids much more. Recently, a new DHA carrier named Major Family Super domain 2a (MFSD2a) was reported to affect DHA transfer in the brain and it is unknown whether it may explain lipid disturbances.

Objectives: To analyze serum fatty acids in patients with several degrees of $\mathrm{AD}$, and discern whether the DHA level is associated with the MFSD2a level in blood.

Methods: Blood was provided by the Biobank of "Virgen de la Arrixaca" Hospital (Murcia). Three groups of patients were established: 70 healthy controls, 73 subjects diagnosed with AD GDS-4,

XVII Conference of SEÑ and X Meeting of ACCA

and 51 subjects with AD GDS-6. Serum fatty acids were analyzed by gas chromatography. MFSD2a protein was analyzed by Western-blotting from blood. The ANOVA test was used for statistical analysis.

Results: We observed a significant reduction of DHA in the serum of AD patients $(3.44 \pm 0.12 \mathrm{a} \%$ control, $3.06 \pm 0.10 \mathrm{a} \%$ GDS$4,2.93 \pm 0.15 \mathrm{~b} \%$ GDS- $6, \mathrm{p}=0.011)$. In addition there was a significant reduction in the $\%$ of long-chain polyunsaturated fatty acids (LC-PUFA) n-3 ( $\mathrm{p}=0.006)$, whereas no differences were found for LC-PUFA n-6 (9.55 $\pm 0.2 \%$ control, 9.32 $\pm 0.23 \%$ GDS-4, 9.68 $\pm 0.3 \%$ GDS-6, $\mathrm{p}=0.616)$. Monounsaturated fatty acids also increased in $\mathrm{AD}$ patients $(23.7 \pm 0.5 \mathrm{a} \%$ control, $25.76 \pm 0.4 \mathrm{ab} \%$ GDS- $4, \%$ $25.65 \pm 0.7 \mathrm{~b} \%$ GDS-6, $\mathrm{p}=0.005)$. MFSD2a in blood did not correlate to DHA\% ( $\mathrm{r}=-0.05, \mathrm{p}=0.500)$ or other major fatty acids.

Conclusions: $\mathrm{AD}$ patients have a marked reduction of omega-3 fatty acids in serum, but this is not associated with the MFS$\mathrm{D} 2 \mathrm{a}$ level in blood. Other important fatty acids are altered in $\mathrm{AD}$, but not omega- 6 fatty acids.

Conflict of interest: Authors declare no conflict of interest.

Key words: docosahexaenoic acid / Alzheimer's disease.

\section{9

ASSOCIATION OF AN OBESITY-PREDISPOSING
GENETIC RISK SCORE WITH A SET OF METABOL-
IC AND INFLAMMATORY TRAITS IN A COHORT
OF SPANISH CHILDREN

${ }^{(1)}$ A. Anguita-Ruiz; (2) A. Rupérez; (1)J. Olza; (2) L. Moreno; ${ }^{(1)} A$. Gil; ${ }^{(2)} G$. Bueno; ${ }^{(3)}$ R. Leis; ${ }^{(1)}$ C. Aguilera.

(1)Department of Biochemistry and Molecular Biology II. Institute of Nutrition and Food Technology "Jose Mataix". Centre of Biomedical Research. University of Granada. Granada. Spain; (2)Health Sciences Institute in Aragon. Pediatric Department. Lozano Blesa University Clinical Hospital. University of Zaragoza. Zaragoza. Spain; ${ }^{(3)}$ Unit of Investigation in Nutrition, Growth and Human Development of Galicia. Paediatric Department. Clinic University Hospital of Santiago. Santiago de Compostela. Spain.

Introduction: In a previous study, we demonstrated how an obesity-predisposing GRS, based on 44 GWAS-significant SNPs, was strongly associated with the BMI $\left(\mathrm{P}=1 \times 10^{\wedge}-7\right)$ in a cohort of Spanish children, and performed well as a predictive tool for the identification of high-risk individuals. On the contrary, the relationship between this GRS and other obesity-related metabolic traits was not studied.

Objectives: To study the relationship between the constructed GRS and other obesity-related metabolic and inflammatory traits.

Methods: The population was composed of 196 normal-weight, 139 overweight and 362 obese children. A total of 25 biochemical markers were measured in the population and association with the GRS was investigated by means of multiple linear regression models (including GRS as both a BMI-adjusted marginal term and $\mathrm{BMI}^{\star} \mathrm{GRS}$ interaction term). Highlighted among the analyzed measurements are lipid and glucose metabolism biomarkers, 
adipokines, as well as cardiovascular-risk and pro-inflammatory biomarkers.

Results: At a significance level of alpha $=0.05$, a model adjusted for BMI, Sex, Age, Puberty, and Origin showed a statistically significant association $(\mathrm{P}=0.002)$ between the GRS and TNFa levels (ng/L). The size effect of the association was estimated as a 0.81 increase in individuals belonging to the third quartile (Q3) of the GRS in comparison with first quartile children. Regarding marginal effects, no other trait besides TNFa showed statistically significant results after BMI-adjustment. In contrast, in regression models including a $\mathrm{BMI}^{\star} \mathrm{GRS}$ interaction term, three significant associations were identified, two of them when the GRS was treated as a continuous variable and related to Leptin(ug/L) and IL$8(\mathrm{ng} / \mathrm{L})$ levels $(\mathrm{P}=0.046, \mathrm{Beta}=1.04$ and $\mathrm{P}=0.038$, Beta $=0.32$ respectively). The third one was identified when GRS was dummy-coded (quartilized) and was related to TNFa levels (ng/L) $(\mathrm{P}<0.001$ and Beta $=0.16$ for the interaction term $\left.\mathrm{BMI}^{\star} \mathrm{GRS}-\mathrm{Q} 3\right)$.

Conclusions: Levels of inflammatory markers are higher in obese children with a high-risk genetic profile compared to obese children with a low-risk genetic background.

Conflict of interest: Authors declare no conflict of interest.

Key words: childhood obesity / GRS / inflammatory pathways / GWAS / genetics.

\section{0 \\ DIFFERENCES IN THE SERUM KINETIC BEHAV- IOR OF ORGANIC AND CONVENTIONAL WHOLE RED GRAPE PHENOLIC METABOLITES.}

\author{
L. Iglesias-Carres; A. Mas-Capdevila; F.I. Bravo; A. Arola-Arnal; B. \\ Muguerza. \\ Departament de Bioquímica i Biotecnologia. Nutrigenomics \\ Research Group. Tarragona. Spain.
}

Introduction: Phenolic compounds are reported to have a wide range of health effects, which are mainly attributed to their metabolites. Factors such as the food matrix, which may be affected by cultivation conditions, are known to affect the bioavailability of phenolic compounds. Notably, the phenolic profile of grapes is known to vary depending on the cultivation system. Therefore, it is plausible that the bioavailability of phenolic compounds from organic (OG) and conventional (CG) Grenache grapes differs.

Objectives: This study aimed to investigate the kinetic differences in the bioavailability of OG and CG phenolic compounds.

Methods: Whole red OG or CG at a dose of $64.6 \mathrm{mg} \mathrm{GAE} /$ $\mathrm{Kg}$ weight was administered to 8-hour starved 30 -week-old male Wistar rats ( $\mathrm{n}=6$, each). Serum was recollected before and 2, 4, 7, 24 and 48 hours after oral administration. Serum phenolic compounds were purified and concentrated by micro-solid phase extraction ( $\mu$-SPE) and analyzed by HPLC-ESI-MS/MS.

Results: The phenolic profile of OG and CG was very similar, but several differences were found in terms of quantity of specific polyphenols (e.g., catechin). In the serum, different metabolic products were detected, and their kinetic behavior was very similar between groups. Notably, two serum maximum peaks appeared, one at $2 \mathrm{~h}$ and the other at $24 \mathrm{~h}$. However, OG-administered rats showed higher concentrations at $2 \mathrm{~h}$ in all the studied metabolic compounds except flavonoid metabolites. In contrast, CG-administered rats showed a higher concentration at $24 \mathrm{~h}$ of all metabolites. This suggests an increased small intestine metabolism of OG phenolics and a more extensive microbial metabolism of CG phenolics.

Conclusions: This study demonstrates that grape variety can have an important impact on the bioavailability of grape phenolic compounds, which could have relevant implications in their biological effects.

Conflict of interest: Authors declare no conflict of interest.

Acknowledgements: Study supported by MINECO (AGL2013-49500-EXP, AGL2016-77105-R).

Key words: flavonoids / grapes / metabolism / polyphenols. 


\section{Poster Abstract Presentations}

061

\section{ROLE OF FIBROBLAST GROWTH FACTOR 21 (FGF21) IN THE METABOLIC RESPONSE TO FASTING}

\author{
Ú. Martínez Garza; V. Sandoval Sandoval; H. Beril; A. Carrilho Do \\ Rosário; H. Sanz; D. Haro; P. Marrero; J. Relat. \\ Department of Nutrition, Food Sciences and Gastronomy. \\ Faculty of Pharmacy and Food Sciences. Torribera Campus. \\ Barcelona. Spain.
}

Introduction: Fibroblast Growth Factor 21 (FGF21) is a hepatoquin with important effects on lipid and glycemic metabolism and has been described as an essential component in the metabolic adaptation to fasting. However, the specific role of FGF21 in this adaptive state of nutrient deprivation is not yet clear.

Objectives: To analyze the metabolic pathways of adaptation to fasting that are dependent on FGF21.

Methods: Male mice C57BL6 / J of 10 weeks of age with specific hepatic depletion of FGF21 (LFKO) and their respective control model (LoxP) were subjected to $24 \mathrm{~h}$ of fasting. They were sacrificed together with libitum-fed mice, and the expression of the main genes involved in the metabolic response to fasting was analyzed by qPCR.

Results: The results indicate that after 24 hours of fasting there was an induction of FGF21 expression at the hepatic level. In addition, two different patterns of expression were observed, giving rise to the presence of metabolic pathways whose activation depends on the presence of FGF21 (gluconeogenesis) and others that do not depend on the presence of hepatic FGF21 (ketogenesis).

Conflict of interest: Authors declare no conflict of interest.

Acknowledgements: SAF2013-41093 Ministry of Economy and Competitiveness, 2014SGR916 Generalitat de Catalunya, Ajut Associació Catalana de Diabetis a la recerca en diabetes 2017, National Commission of Scientific and Technological Research CONICYT-Chile, National Council of Science and Technology CONACYT-Mexico.

Key words: fgf21 / fasting / fed / metabolism.

\section{2}

ASSOCIATION OF THE EXTRACELLULAR P53 PROTEIN PROFILE WITH OBESITY IN THE MEXICAN ADULT POPULATION: A PILOT STUDY

\author{
${ }^{(1)}$ O. González; (2)L. Yáñez; ${ }^{(2)}$ O. Galicia; (1)D. Gaytán; (1)J. Ríos; ${ }^{(1)} P$. \\ Algara; ${ }^{(1)}$ C. Victoria. \\ (1)Facultad de Enfermería y Nutrición, Universidad Autónoma de \\ San Luis Potosí. San Luis Potosí. México; ${ }^{(2)}$ Facultad de Medicina, \\ Universidad Autónoma de San Luis Potosí. San Luis Potosí. \\ México.
}

Introduction: The conventional functions of p53 include cell-cycle arrest, senescence and apoptosis. New roles for p53 in regulating cell metabolism are receiving increasing attention. For instance, p53 regulates various targets to affect insulin resistance and reduce glucose metabolism through glycolysis, a metabolic pathway that favors tumor growth. The ability of p53 to regulate metabolism is a powerful feature of $\mathrm{p} 53$ biology that can both promote cell survival and act as a check on the inappropriate proliferation of cancer cells.

Objectives: To evaluate the profile of extracellular $\mathrm{p} 53$ protein in blood of obese young adults.

Methods: A descriptive cross-sectional study, registered at the Ethics Committee, was carried out on 58 patients of 18-40 years, both sexes, with previous consent. Sedentary and obese parents were included. Volunteers with carcinoma, low weight, overweight, endocrinological diseases, diagnosed syndromes, treatment for obesity, pregnancy or lactation were excluded. Volunteers were classified in normal weight, control and obesity groups according to the WHO. Serum glucose, cholesterol, triglycerides, HDL, LDL, and p53 were analyzed by an ELISA kit, and scores greater than 1 were considered as indicative of $\mathrm{p} 53$ presence. The analysis was performed with PASW Statistics 22.0 for descriptive statistics, binary logistic regression, Pearson linear correlation and Chi 2 with 95\% reliability.

Results: The proportion of obese patients with absence of p53 was $60.7 \%(\mathrm{p}=0.009)$. In the binary logistic regression an $\mathrm{OR}=$ 4.250 and I.C. of $95 \%$ [1,402 - 12,884] were found. These data reveal that patients expressing p 53 were 250 -fold more likely to conserve normal weight than those who did not. p53 was not related to the lipid profile or glucose levels.

Conclusions: The absence of p53 in the extracellular concentration in obese young patients supports the need to continue studying the expression of this gene and its relationship with chronic diseases.

Conflict of interest: Authors declare no conflict of interest.

Key words: p53 / obesity / adult / Mexican. 


\section{3}

PREGNANCY-INDUCED HYPERTENSION IS ASSOCIATED WITH METABOLIC ABNORMALITIES AND INCREASED PRO-INFLAMMATORY BIOMARKERS IN OFFSPRING

\author{
${ }^{(1)}$ A.I. Rupérez; ${ }^{(2)}$ L. Trujillano; ${ }^{(3)}$ E.M. González-Gil; ${ }^{(4)}$ R. Leis; ${ }^{(5)}$ M. Gil- \\ Campos; ${ }^{(6)}$ C.M.Aguilera; ${ }^{(1)}$ L.A. Moreno; ${ }^{(2)}$ G. Bueno.
}

\begin{abstract}
(1)Department of Physiatry and Nursery. Growth, Exercise, Nutrition and Development Research Group. University of Zaragoza. Zaragoza. Spain. (2)Paediatric Department. Lozano Blesa University Hospital. University of Zaragoza. Zaragoza. Spain. ${ }^{(3)}$ Growth, Exercise, Nutrition and Development Research Group. University of Zaragoza. Zaragoza. Spain. ${ }^{(4)}$ Unit of Investigation in Nutrition, Growth and Human Development of Galicia. Paediatric Department. University of Santiago de Compostela. Santiago de Compostela. Spain. ${ }^{(5)}$ Paediatric Research and Metabolism Unit. Reina Sofia University Hospital. Maimonides Institute for Biomedical Research. Córdoba. Spain. ${ }^{\left({ }^{6}\right)}$ Department of Biochemistry and Molecular Biology II. Institute of Nutrition and Food Technology "José Mataix". University of Granada. Granada. Spain.
\end{abstract}

Introduction: Gestational metabolic alterations such as pregnancy-induced hypertension (PIH) are known to increase the risk of obesity and metabolic alterations in offspring. However, it is unclear whether these associations are accompanied by an alteration of pro-inflammatory and cardiovascular disease biomarkers.

Objectives: To investigate whether the higher metabolic risk in prepubertal children born to mothers with $\mathrm{PIH}$ is accompanied by increased circulating concentrations of pro-inflammatory and cardiovascular disease biomarkers.

Methods: A total of 334 children (184 males, 232 overweight/ obese) recruited in three Spanish cities participated in the study. Presence of PIH was registered along with birth-related information; anthropometry and BP were measured, and plasma biomarkers were analyzed. Linear regression adjusted by age and recruitment center and decision tree classification analysis were employed to assess the relationships between PHD and the other evaluated parameters.

Results: Children born to mothers with PIH had higher odds of being born through $\mathrm{C}$-Section $(\mathrm{P}=0.020)$, preterm $(\mathrm{P}=0.009)$ or macrosomic ( $\geq 4000 \mathrm{~g})(\mathrm{P}<0.001)$, having overweight/obesity $(\mathrm{P}=0.008)$ and being metabolically unhealthy $(\mathrm{P}=0.048)$. They also showed significantly higher weight, BMI, BMI Z-score and diastolic $\mathrm{BP}$ values (all $\mathrm{P}<0.05$ ), as well as increased total plasminogen activator-inhibitor-1 (tPAI-1) $(\mathrm{P}=0.038)$ and matrix metalloproteinase-9 (MMP-9) $(\mathrm{P}=0.003)$ concentrations. Decision tree analysis (estimation error $=8.2 \%$ ) revealed an association between $\mathrm{PIH}$ and high $\mathrm{BMI}\left(>25 \mathrm{~kg} / \mathrm{m}^{2}\right)$ among those with low HDL-c $(\leq 35 \mathrm{mg} /$ $\mathrm{dL})$, as well as increased tPAI-1 $(>44.66 \mu \mathrm{g} / \mathrm{L})$ and MPO $(>34.17$ $\mu \mathrm{g} / \mathrm{L})$ concentrations among those with higher HDL-c concentrations (>35 mg/dL).

Conclusions: The offspring of mothers with $\mathrm{PIH}$ have an increased risk of overweight/obesity during childhood, which is accompanied by metabolic alterations, including increased cir- culating concentrations of pro-inflammatory and cardiovascular disease biomarkers tPAI-1 and MMP-9. Algorithmic analysis provided additional information to these findings, suggesting an interaction between the altered biomarkers and offspring HDL-c concentrations.

Conflict of interest: Authors declare no conflict of interest.

Key words: pregnancy / hypertension / offspring / biomarkers / inflammation.

\section{4 \\ MFSD2A PROTEIN IN THE BLOOD OF ALZHEIM- ER'S PATIENTS DECREASES IN THE ADVANCED STAGE OF THE DISEASE}

\begin{abstract}
${ }^{(1)}$ M. Sánchez-Campillo; ${ }^{(1)}$ M.J. Ruiz-Pastor; ${ }^{(2)} J$. Marín-Muñoz; ${ }^{(2)}$ F. Noguera-Perea; ${ }^{(3)}$ A.J. Ruiz-Alcaraz; ${ }^{(2)} C$. Antunez; ${ }^{(1)} E$. Larqué.

${ }^{(1)}$ Department of Physiology. Faculty of Biology. Campus Mare Nostrum. University of Murcia. Espinardo. Spain; ${ }^{(2)}$ Dementia Unit. Hospital Clinico Universitario Virgen de la Arrixaca. El Palmar. Spain; ${ }^{(3)}$ Department of Biochemistry, Molecular Biology $B$ and Immunology. Faculty of Medicine. University of Murcia. Espinardo. Spain.
\end{abstract}

Introduction: Alzheimer's disease $(\mathrm{AD})$ is a progressive neurodegenerative disorder and the most common cause of dementia in the elderly. The Global Deterioration Scale (GDS) provides an overview of 7 different stages of cognitive function for those suffering AD. Stages 1-3 are pre-dementia and 4-7 are the dementia stages. On the other hand, DHA is involved in a variety of physiological processes, including memory formation, synaptic membrane functions, and neuroprotection. Meta-analyses showed lower brain and circulatory DHA levels in AD patients. The primary carrier for the uptake of DHA and other long-chain fatty acids into the brain was recently characterized as the Major Facilitator Superfamily Domain containing 2A (MFSD2a).

Objectives: To detect if the MFSD2a level is altered in AD patients in tissues such as blood, which provides an easy-to-obtain source of non-invasive human samples.

Methods: Three groups were established for the analysis of MFSD2a in blood: 70 healthy controls, 69 subjects diagnosed with $\mathrm{AD}$ at GDS4, and 53 subjects diagnosed with AD at GDS6. The MFSD2a expression was analyzed by Western-blotting and results expressed as mean \pm SEM. Differences between GDS1, GDS4 and GDS6 groups were evaluated by ANOVA and post hoc Bonferroni analyses.

Results: We report for the first time a greater decline of MFSD2a levels in the blood of AD patients of the GDS6 group than in other groups (Control 0.77 $\pm 0.08 \mathrm{a}$, GDS4 0.69 $\pm 0.08 \mathrm{ab}$ and GDS6 $0.48 \pm 0.04 \mathrm{~b}, \mathrm{p}<0.05)$. Due to the importance of these results, we are currently analyzing MFSD2a levels in the brain in a subset of these subjects ( 10 controls and 10 subjects GDS-6) in order to confirm if MFSD2a in blood could be used as a biomarker of MFSD2a in the central nervous system. 
Conclusions: MFSD2a presence in blood is lower in advanced stages of $\mathrm{AD}$, which could affect DHA transport in this pathology.

Conflict of interest: Authors declare no conflict of interest.

Key words: alzheimer / MFSD2A / DHA / biomarker / transport.

\section{5}

\section{MATERNAL PRE-PREGNANCY BODY MASS IN- DEX IMPRINTS A GUT MICROBIAL COMMUNITY COMPOSITION WITH A SPECIFIC FUNCTIONAL- ITY}

\author{
${ }^{(1)}$ C. Campoy; ${ }^{(1)}$ T. Cerdó; ${ }^{(2)}$ A. Ruiz; ${ }^{(3)}$ R. Jauregui; ${ }^{(4)}$ M. von Bergen M; \\ ${ }^{(2)}$ A. Suarez. \\ (1)Department of Pediatrics. University of Granada. Granada. \\ Spain; ${ }^{(2)}$ Department of Biochemistry and Molecular Biology. \\ University of Granada. Granada. Spain; ${ }^{\left({ }^{3}\right)}$ AgResearch Grasslands, \\ Tennent Drive, Palmerston North, New Zealand; (4)Department of \\ Molecular System Biology, Helmholtz Centre for Environmental \\ Research- UFZ, Leipzig, Germany.
}

Introduction: Mothers have been shown to transmit distinct gut microbial communities to their offspring, depending on maternal pre-pregnancy weight.

Objectives: Since children born to obese mothers are at increased risk of obesity, we hypothesized that neonatal acquisition of microbiota may also translate into functional ecosystem restructuring that may contribute to obesity development.

Methods: We tested whether gut microbial composition and functionality at 6-months of age (stool samples, $\mathrm{n}=68$ ) were associated with maternal pre-pregnancy body mass index using $16 \mathrm{~S}$ rRNA gene sequencing, metaproteomics and glycosyl hydrolase activities.

Results: We observed significant differences in gut community structure and composition among groups. Compared to infants born to overweight mothers, infants born to obese mothers had a significantly different gut microbial community structure (unweighted and weighted UniFrac distances PERMANOVA, $\mathrm{p}<0.024$ and $\mathrm{p}<0.004)$, enriched in Firmicutes and depleted in Proteobacteria. The gut microbiota of infants born to normal weight mothers was enriched in Staphylococcus $(p<0.021)$, Coprobacillus $(\mathrm{p}<0.001)$, Paraprevotella $(\mathrm{p}<00.39)$ and unclassified_Alphaproteobacteria $(p<0.004)$, while Yokenella $(p<0.011)$, Turicibacter $(\mathrm{p}<0.045)$ and unclassified_Chitinophagaceae $(\mathrm{p}<005)$ increased in the microbiota of infants born to overweight mothers. These microbial signatures resulted in over-representation of functions involved in nucleotide transport and metabolism and in energy production and conversion metabolism. We observed increased abundances of translation elongation factor GTPase (COG0480), acetylornithine deacetylase (COG0624), maltooligosaccharide transport system substrate-binding protein (COG2182) and b-glucosidase (COG1472) in the gut microbiota of infants born to obese mothers. Since our previous work demonstrated an increased glycosidase activity in the gut microbiota of obese subjects that correlated with insulin resistance, we tested and observed increased gut microbial b-galactosidase, b-glucosidase and a-glucosidase activities in infants born to overweight and obese mothers.

Conclusions: Our findings indicate that excess maternal pre-pregnancy weight associated with differences in gut microbial metabolic performances may alter metabolic health in offspring.

Conflict of interest: Authors declare no conflict of interest.

Key words: obesity / BMI / infancy / microbiota.

\section{6 \\ LOW DOSE OF CONJUGATED LINOLENIC ACID REDUCES WEIGHT GAIN, INCREASING ENERGY EXPENDITURE WITHOUT ENHANCING HEPATIC STEATOSIS IN CAFETERIA DIET-FED RATS}

\author{
M. Martin; H. Palacios; C. Badé; G. Aragonés; B. Muguerza.
}

Departament de Bioquímica i Biotecnologia. Nutrigenomics Research Group. Universitat Rovira i Virgili. Tarragona. Spain.

Introduction: Conjugated linoleic acid (CLA) is a dietary supplement that has been shown to reduce body weight and fat mass in mammals. However, some isomers such as trans-10 and cis-12 could enhance the hepatic lipid content, depending on the CLA content in diet, feeding length, physiological condition and animal species.

Objectives: As we hypothesize that beneficial and detrimental effects of CLA could be dose-dependent in mammals, we conducted a study with three low doses of CLA (100, 200 and $300 \mathrm{mg} /$ $\mathrm{kg} /$ day) in diet-induced obese rats, aiming to find one that most improves their metabolic status without detrimental effects on the liver.

Methods: Eight-week-old Wistar rats were fed a cafeteria-diet for 12 weeks and were orally supplemented with different doses of CLA in the last 3. Body weight was monitored weekly and calorimetric studies were carried out in the last week of treatment. Additionally, biochemical and histological analysis of the liver was performed at the end of the treatment.

Results: Consumption of $100 \mathrm{mg} / \mathrm{kg} /$ day of CLA significantly reduced body weight gain with an improved plasma lipid profile. Notably, this body weight regulation was accompanied by a lower respiratory quotient, increased energy expenditure and higher lipid oxidation. In contrast, no significant changes in body weight were observed at doses of 200 or $300 \mathrm{mg} / \mathrm{kg} /$ day. Furthermore, $100 \mathrm{mg} / \mathrm{kg}$ supplemented rats did not manifest hepatic signs of enhanced fat content, inflammation or fibrosis compared to the control group.

Conclusions: Our results demonstrate that the physiological effects of CLA supplementation rely on a precise dose to provide the desired impact on the metabolic status. Most studies have been performed using higher doses of CLA, which may explain why 100 $\mathrm{mg} / \mathrm{kg} /$ day did not induce hepatic deterioration.

Acknowledgements: This work was supported by grant numbers AGL-2013-40707-R and AGL2016-77105-R from the Spanish Government. 
Conflict of interest: Authors declare no conflict of interest.

Key words: conjugated linoleic acid / body weight.

\section{7 \\ VALIDATION OF DRIED BLOOD SPOT CARDS TO DETERMINE APPLE PHENOLIC METABO- LITES AFTER A HUMAN ACUTE INTAKE OF RED- FLESHED APPLE SNACK}

L. Rubió; S. Yuste; A. Macià; M.P. Romero; I. Ludwig; M.J. Motilva.

Departamento de Tecnología de Alimentos. Universidad de Lleida. Lleida. Spain.

Introduction: Dried blood spot (DBS) has established itself as an innovative sampling technique where capillary blood obtained by finger-pricking is spotted onto absorbent paper for quantitative biomarker assessment. However, its application for dietary polyphenol pharmacokinetics in human blood has been poorly studied.

Objectives: To develop an analytical method to analyze the main circulating phenolic metabolites after an acute intake of a red-fleshed apple snack as a source of different subclasses of phenolic compounds.

Methods: The method was based on the combination of DBS and liquid chromatography coupled to tandem mass spectrometry. Capillary blood by finger prick was compared to venous blood by venipuncture in ten healthy volunteers from 0 to $24 \mathrm{~h}$. The pharmacokinetics of the main apple phenolic metabolites in whole blood was also compared to their corresponding venous plasma samples.

Results: The main phenolic metabolites detected in whole blood and plasma were phase-II and microbial metabolites derived from phenolic acids, dihydrochalcones and anthocyanins. The self-sampling of blood by study participants using DBS allowed collecting those late time points $(12 \mathrm{~h})$ to assess the colonic metabolism of phenolic compounds, which are normally missing with venipuncture. The concentrations did not differ significantly between venous blood and capillary blood. Moreover, no significant differences were found between blood and plasma levels, suggesting that these metabolites do not accumulate in human blood cells.

Conclusions: These results indicate that the developed method based on DBS appears to be a suitable alternative to the classic invasive venipuncture for the determination of circulating phenolic metabolites in nutritional postprandial studies, allowing a deeper assessment of the colonic metabolism of phenolic compounds.

Conflict of interest: Authors declare no conflict of interest.

Key words: apple / pharmacokinetics / polyphenols / self-sampling / tandem-MS. 


\section{Track 3: Food Bioactive Compounds}

\section{Nutritionals of Metabolism}

\author{
Reviewers: \\ Concepción Aguilera García \\ Universidad de Granada \\ Lluís Arola Ferrer \\ Universitat de Rovira i Virgili \\ Margarita Castell Escuer \\ Universitat de Barcelona \\ Ángel Gil Hernández \\ Universidad de Granada \\ Rosa M. Lamuela Raventós \\ Universitat de Barcelona
}

Elvira Larqué Daza

Universidad de Murcia

Alfredo Martinez Hernández

Universidad de Pamplona

Joana M. Planas Rosselló

Universitat de Barcelona

Maria del Puy Portillo Baquedano

Universidad del País Vasco

\section{Oral Abstract Presentations}

\section{8}

\section{CONTRIBUTION OF QUERCETIN METABOLITES TO THE TRIGLYCERIDE-LOWERING EFFECT OF THE PARENT COMPOUND IN 3T3-L1 ADIPO- CYTES}

\author{
${ }^{(1)}$ I. Eseberri; ${ }^{(1)} \mathrm{A}$, Lasa; ${ }^{(1)} \mathrm{J}$. Miranda; ${ }^{(1)} \mathrm{A}$. Mosqueda-Solís; ${ }^{(2)} \mathrm{S}$. \\ González-Manzano; ${ }^{(2)}$ C. Santos-Buelga; ${ }^{(1)}$ M.P. Portillo.
}

\begin{abstract}
(1)Grupo Nutrición y Obesidad. Departamento de Farmacia y Ciencias de los Alimentos. Universidad del País Vasco - Euskal Herriko Unibertsitatea (UPV-EHU). Vitoria-Gasteiz. Spain; (2) Grupo de Investigación en Polifenoles (GIP-USAL). Facultad de Farmacia. Universidad de Salamanca. Salamanca. Spain.
\end{abstract}

Introduction: Quercetin $(\mathrm{Q})$ is a potential body fat-lowering flavonoid, as has been confirmed by several cell culture and ani-

\footnotetext{
KARGER XVII Conference of SEÑ and X Meeting of ACCA
}

mal studies. Research in humans is still scarce. However, due to its rapid metabolism, it is uncertain if the observed anti-obesity properties of $\mathrm{Q}$ in in vivo experiments can be exclusively attributed to Q. According to the literature, the most predominant metabolites in plasma are isorhamnetin (ISO), tamarixetin (TAM), quercetin-3-O-glucuronide (3G) and quercetin-3-O-sulfate (3S).

Objectives: To compare the triglyceride-lowering effect of predominant Q metabolites with that of Q in 3T3-L1 pre-adipocytes as well as in mature adipocytes, and to describe their potential mechanism of action.

Methods: 3T3-L1 pre-adipocytes were treated during differentiation and mature adipocytes on day 12 of differentiation for 24 hours with $0.1,1$ and $10 \mu \mathrm{M}$ of Q, ISO, TAM, 3G, 3S and 3S and quercetin-4-O-sulfate (4S) mixture (3S+4S). Triacylglycerol (TG) content in both treatments was measured spectrophotometrically and gene expression was analyzed by RT-PCR.

Results: In mature adipocytes, the $3 \mathrm{~S}$ metabolite decreased the TG content at 1 and $10 \mu \mathrm{M}$ and the $3 S+4 S$ mixture at $10 \mu \mathrm{M}$. Q reduced TG at 1 and $10 \mu \mathrm{M}$. The treatment with $10 \mu \mathrm{M}$ of $3 \mathrm{~S}$ tended to reduce hsl lipase expression and increased deacetylase sirtuin 1 (sirt1) and tumor protein p53 (trp53) mRNA levels. The $3 S+4 S$ treatment increased trp53 gene expression. During differentiation, $\mathrm{Q}$ and ISO reduced the TG content at $10 \mu \mathrm{M}$. ISO increased trp53 expression and tended to reduce that of ppar $\gamma$.

Conclusions: The $3 \mathrm{~S}$ metabolite can contribute to the in vivo delipidating effect of $\mathrm{Q}$ by inducing apoptosis in mature adipocytes. In contrast, considering that ISO needs a dose far from that found in plasma to reduce TG, this metabolite can be discarded as a contributor to the anti-obesity effect of $\mathrm{Q}$.

Conflict of interest: Authors declare no conflict of interest.

Key words: quercetin / metabolites / adipocytes / adipogenesis / triglyceride.

\section{9 \\ MEDITERRANEAN DRIED FRUITS ARE A RICH SOURCE OF PHENOLIC COMPOUNDS

\author{
${ }^{(1,2)}$ M. Marhuenda-Muñoz; ${ }^{(1)} D$. Escobar-Avello; ${ }^{(1)} M$. Trius; ${ }^{(1)} J . F$. \\ Rinaldi de Alvarenga; ${ }^{(1,2)}$ A. Vallverdú-Queralt; ${ }^{(1,2)}$ R.M. Lamuela- \\ Raventós. \\ (1)Department of Nutrition, Food Sciences and Grastronomy. \\ XaRTA. INSA.UB. School of Pharmacy and Food Sciences. \\ University of Barcelona. Barcelona. Spain; ${ }^{(2)}$ CIBEROBN. Instituto \\ de Carlos III. Madrid. Spain.
}

Introduction: Dried fruits (DF) are a form of fruit that have all their bioactive non-volatile compounds concentrated due to a 
dehydration process. They are consumed worldwide and have potential benefits in type 2 diabetes and other diseases related to metabolic syndrome. While epidemiological studies suggest fresh fruit consumption has an important role in the prevention of chronic diseases, studies on DF are limited. The beneficial effects of DF have been attributed to their high fiber and phenolic compound content, but the specific characteristics of the main phenolic compounds have not been thoroughly established yet.

Objectives: To identify the phenolic profile of different commercial DF (raisins, dates, dried apricots and prunes) by LC-ESILTQ-Orbitrap-MS analysis.

Methods: The polyphenols were extracted by grinding the DF and using a mixture of ethanol:water $(80: 20, \mathrm{v} / \mathrm{v})$. Total phenolics were quantified by the Folin-Ciocalteau method. An LC-ESILTQ-Orbitrap-MS system working in negative mode was used for accurate mass measurements. The most intense ions detected in full scan spectrum were selected and identified using Xcalibur 3.0 software.

Results: The total phenolic composition was between 0.247 $1.764 \mathrm{mg} \mathrm{GAE} / \mathrm{g}$, prunes showing the highest concentration, followed by dried apricots. Similar, but lower, concentrations were observed in raisins and dates. A number of phenolic compounds were identified with high precision mass $(>2 \mathrm{mDa})$. Compounds from the main phenolic families were found: phenolic acids such as gallic and chlorogenic; and flavonoids such as quercetin, catechin and epicatechin, among others.

Conclusions: DF could be an important source of phenolic compounds in the Mediterranean diet. A daily intake of $30 \mathrm{~g}$ of these fruits would represent a consumption of 7.41-52.92 mg polyphenols.

Conflict of interest: Authors declare no conflict of interest.

Key words: prune / date / apricot / raisin /orbitrap.

\section{0 \\ GLOBIN HYDROLYSATES PREVENT FAT DEPOSI- TION IN AN OBESITY MODEL}

\author{
${ }^{(1,2)}$ C. Rosell-Cardona; $(1,3)$ L. Miró; ${ }^{(1,2)}$ A. Pérez-Bosque ${ }^{(1,2)}$ M. Moretó;
}

(3) J. Polo; ${ }^{(1,2)}$ C. Amat.

(1)Departament de Bioquímica i Fisiologia. Facultat de Farmàcia i Ciències de l'Alimentació. Universitat de Barcelona. Barcelona. Spain; ${ }^{(2)}$ Institut de Recerca en Nutrició i Seguretat Alimentària. Universitat de Barcelona. Barcelona. Spain; ${ }^{(3)}$ APC-Europe, SLU. Granollers. Spain.

Introduction: The prevalence of overweight and obesity is increasing worldwide, leading to dramatic increases in health complications such as hyperlipidemia, heart disease and type II diabetes mellitus. Much attention has been focused on functional protein hydrolysates, which might have beneficial effects on nutrient metabolism.

Objective: To analyze the effects of globin digest (GD) on the absorption of triglycerides and fat deposition.

Methods: C57BL/6 male mice were used. In a short-term experiment, three doses of GD $(0.4,2$ and $4 \mathrm{~g} / \mathrm{kg} \mathrm{BW})$ were orally administered $30 \mathrm{~min}$ after animals had received olive oil $(5.5 \mathrm{~g} / \mathrm{kg}$ BW). Plasma concentration of triglycerides (TG) was determined 2 and $4 \mathrm{~h}$ post-administration. In a long-term experiment, three different diets were tested (for 2 months): control diet (CTL, $10 \%$ energy from fat); high fat diet (HF, $40 \%$ ); and HF diet supplemented with $0.4 \%$ of GD. After sacrifice, liver, testicles, kidneys and perirenal fat pads were weighed. Epididymal fat pads were removed, and a histological analysis was carried out to measure the adipocyte size.

Results: Two hours after the administration of olive oil there was a marked increase in plasmatic TG, which returned to basal levels $2 \mathrm{~h}$ later. GD prevented the rise in plasmatic TG, lowering the concentration to $20-41 \%$ of the control values. The administration of the HF diet increased body weight, perirenal and epididymal fat pads, as well as the sectional area of adipocytes. The dietary supplementation with GD at $0.4 \%$ for 2 months mitigated most of the effects observed in animals fed a HF diet.

Conclusions: Globin hydrolysates may contribute to the prevention of obesity and metabolic diseases related with lipid metabolism.

Conflict of interest: C.R-C., A.P-B., M.M. and C.A. declare no conflict of interest. J.P. and L.M. are employed by APC Europe. Funding sponsors had no role in the study (FBG 308133).

Key words: protein hydrolysates / adiposity / diet.

\section{1 \\ ROLE OF COCOA THEOBROMINE IN THE METAB- OLISM OF YOUNG LEWIS RATS}

\author{
M. Camps-Bossacoma; M. Massot-Cladera; M. Abril-Gil; S. Saldaña- \\ Ruiz; A. Franch; F.J. Pérez-Cano; M. Castell. \\ Secció de Fisiologia. Departament de Bioquímica i Fisiologia. \\ INSA.UB. Facultat de Farmàcia i Ciències de l'Alimentació. \\ Universitat de Barcelona. Barcelona. Spain.
}

Introduction: In recent years, the potential of cocoa's metabolic effects has increasingly attracted attention. Although some studies have suggested the implication of polyphenols in such effects, we hypothesize here that theobromine, a methylxanthine found in cocoa, could also have an important metabolic role.

Objectives: To establish the role of theobromine in the metabolic effects of cocoa.

Methods: 3-week-old rats were fed for 18 days with a reference diet (RF), a diet containing $10 \%$ cocoa (CC), or a diet with $0.25 \%$ theobromine (TB), the same amount of theobromine provided by the cocoa diet. Throughout the study, body weight and food intake were quantified. At day 18, rats were food-deprived overnight and tissue samples were collected. Body length and organ weight were determined. Additionally, plasmatic metabolic hormones and biochemical variables were assessed, either by a Bio-Plex Pro ${ }^{\mathrm{Tm}}$ Diabetes Assay (Bio-Rad) or by several colorimetric kits.

Results: No differences in food intake were detected, although the animals from the CC and TB groups had a lower body mass index than those from the RF group. After 18 days of the nutritional intervention, rats from the $\mathrm{CC}$ and $\mathrm{TB}$ groups had lower relative 
retroperitoneal fat and liver weights, as well as a higher relative intestinal tissue weight than the RF group. Additionally, both diets increased plasma ghrelin levels, whereas they decreased glucagon levels without affecting insulin concentrations. CC and TB diets increased glucose and HDL-cholesterol levels and decreased those of plasma and hepatic triglycerides. There were no differences in most of the measured variables between the CC and TB groups.

Conclusions: Although previous studies have ascribed the metabolic effects of cocoa to flavonoids, the results obtained here point to theobromine as a key contributor in such effects, at least in healthy rats.

Conflicts of interest: Authors declare no conflict of interest.

Key words: cholesterol / cocoa / glucose / metabolism / theobromine.

\section{2 \\ EFFECT OF RESVERATROL IN MITOCHONDRIAL SYNTHESIS ON THE SKELETAL MUSCLE OF RATS UNDER STANDARD AND ENERGY RESTRICTION FEEDING CONDITIONS}

\author{
${ }^{(1,2)}$ I. Milton-Laskibar; ${ }^{(1,2)}$ L. Aguirre; ${ }^{(1)}$ N. Romo; ${ }^{(3)}$ U. Etxeberria; ${ }^{(2,3)}$ F.I. \\ Milagro; ${ }^{(2,3)}$ J.A. Martínez; ${ }^{(1,2)}$ M.P. Portillo. \\ (1)Nutrition and Obesity Group. Department of Nutrition and \\ Food Science. Faculty of Pharmacy. UPV-EHU. Vitoria. Spain; \\ ${ }^{(2)}$ CIBERobn Physiopathology of Obesity and Nutrition. ISCIII. \\ Spain; ${ }^{(3)}$ Department of Nutrition, Food Sciences and Physiology. \\ Centre for Nutrition Research. University of Navarra. Pamplona. \\ Spain.
}

Introduction: Skeletal muscle (SM) is one of the main determinants of basal metabolism, due to its activity and size. It has been demonstrated that SM is a target-tissue for resveratrol, a polyphenol able to improve mitochondrial function. In fact, this compound has been proposed as an energy restriction (ER) mimetic.

Objectives: To study the effects induced by resveratrol in the SM mitochondrial function of obese rats under standard and ER feeding conditions.

Methods: Rats (6-week-old; $n=27$ ) were fed a high-fat high-sucrose diet for 6 weeks, and then divided into three groups and fed a standard diet for 6 additional weeks: control group (C), resveratrol group (RSV, $30 \mathrm{mg}$ resveratrol/kg/day) and combined group (RR, $30 \mathrm{mg}$ resveratrol $/ \mathrm{kg} /$ day and $15 \% \mathrm{ER}$ ). Carnitine palmitoyltransferase $1 \mathrm{~b}$ (CPT1b) activity was analysed spectrophotometrically. Mitochondrial transcription factor-A (TFAM) protein levels, as well as AMP protein kinase (AMPK) phosphorylation and peroxisome proliferator-activated receptor gamma coactivator $1 a$ (PG$\mathrm{C} 1 \alpha$ ) acetylation were determined by Western blot.

Results: Increased CPT1b activity was found in RSV and RR groups. Lower PGC1 $\alpha$ acetylation was found in the RSV group, whereas AMPK phosphorylation increased in the RR group. Finally, increased TFAM protein expression was observed in the RSV and RR groups.

XVII Conference of SEÑ and X Meeting of ACCA
Conclusions: Increased CPT1b activity suggests that resveratrol induces greater fatty acid oxidation both in standard and ER feeding conditions in SM. PGC1 $\alpha$ activation suggests that this effect is mediated by mitochondrial biogenesis under standard feeding conditions (RSV group). When resveratrol was administered under ER feeding conditions (RR group), AMPK-mediated acetyl CoA carboxylase inhibition (an inhibitor of CPT1b) cannot be ruled out as a mechanism underlying increased fatty acid oxidation. These results demonstrate that the resveratrol-induced effects on mitochondrial function under standard and ER feeding conditions, although similar, are not exactly the same.

Conflict of interests: Authors declare no conflict of interest.

Key words: resveratrol / mitochondria / skeletal-muscle / energy-restriction / rat.

\section{3 \\ COMPARISON BETWEEN THE POLYPHENOL CONTENT IN PICUAL AND HOJIBLANCA EXTRA VIRGIN OLIVE OILS FROM ECOLOGICAL AND CONVENTIONAL CROPPING SYSTEMS}

\author{
${ }^{(1,2)}$ J. Lozano-Castellón; ${ }^{(1)}$ A. López-Yerena; ${ }^{(1)} A$. Olmo-Cunillera; ${ }^{(1,2)}$ P. \\ Quifer-Rada; ${ }^{(1,2)}$ A. Vallverdú-Queralt; ${ }^{\left({ }^{3}\right)} M$. Pérez Bosch; ${ }^{\left({ }^{(4)} B\right.}$. Jiménez; \\ ${ }^{(1,2)}$ R.M. Lamuela-Raventós. \\ (1)Department of Nutrition, Food Sciences and Grastronomy. \\ XaRTA. INSA.UB. School of Pharmacy and Food Sciences. \\ University of Barcelona. Barcelona. Spain; ${ }^{(2)} \mathrm{CIBEROBN}$. Instituto \\ de Carlos III. Madrid. Spain; ${ }^{(3)}$ Laboratory of Organic Chemistry. \\ Department of Nutrition, Food Sciences and Grastronomy. \\ XaRTA. INSA.UB. School of Pharmacy and Food Sciences. \\ University of Barcelona. Barcelona. Spain; ${ }^{(4)}$ Nutrition and Food \\ Science. Toxicology and Forensic Medicine Department. School \\ of Pharmacy. University of Sevilla. Sevilla. Spain.
}

Introduction: Polyphenols are plant secondary metabolites synthesized during stress, and their concentration is affected by agronomic and technological conditions. These compounds are responsible for olive oil stability, flavor and aroma, and also have important health benefits, such as anti-platelet aggregation or vasodilator activity.

Objectives: To determine the differences in olive oil polyphenol content between two types of cropping systems: ecological and conventional. To determine the differences in the olive oil polyphenol profile between two olive varieties: Picual and Hojiblanca.

Methods: Two varieties of olives, Picual and Hojiblanca, were cropped using two methods, ecological and conventional, in two different groves. The olive oil polyphenols were extracted by a liquid-liquid extraction, using hexane for removing the oil and methanol as an extracting solvent. After the extraction, the methanol was evaporated and the extract was reconstituted in water:methanol (8:2). The amount of polyphenols was determined by liquid chromatography coupled to a mass spectrometer (UHPLC-ESIQqQ). Polyphenols were quantified using an external calibration 
curve prepared by spiking refined olive oil with different concentrations of polyphenols.

Results: The ecological olive oil samples contained a higher amount of polyphenols than the conventional ones. There were also differences between the two varieties of olives.

Conclusions: The growing conditions (conventional versus ecological) affected the total polyphenols of olive oils. When plants grow with artificial nutrients, they are supposed to lose their natural defense mechanisms. This may result in reduced disease resistance and diluted contents of minerals, vitamins, and defense-related secondary metabolites, which are considered beneficial for human health. The variations between Picual and Hojiblanca varieties could be caused by genetic differences.

Conflict of interest: Authors declare no conflict of interest.

Key words: EVOO / polyphenols / UHPLC-ESI-QQQ / ecological / conventional.

\section{4}

\section{RELATIONSHIP BETWEEN DOSE AND ANTIHY- PERTENSIVE EFFECT FOR AN ACE INHIBITOR CHICKEN FEET HYDROLYSATE}

\section{A. Mas-Capdevila; L. Iglesias-Carrés; Z. Pons; A. Arola-Arnal; F.I. Bravo; B. Muguerza.}

Nutrigenomics Research Group. Biochemistry and Biotechnology Department. Universitat Rovira i Virgili. Tarragona. Spain.

Introduction: Protein hydrolysates are a source of bioactive peptides able to inhibit angiotensin-converting enzyme (ACE), a key enzyme for blood pressure (BP) control. The antihypertensive effect of chicken feet hydrolysate (Hpp11) obtained after hydrol-

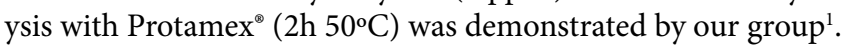

Objectives: To investigate the effect of different doses of Hpp11 in spontaneously hypertensive rats (SHR) and the mechanism involved in this effect.

Methods: Hpp11 was obtained according to Bravo et al. 2017 ${ }^{1}$. Hpp11 at 25, 55 and $85 \mathrm{mg} / \mathrm{kg}$, water and Captopril 50 $\mathrm{mg} / \mathrm{kg}$ were administered, dissolved in water, to male 17-22-weekold SHR. Systolic blood pressure (SBP) was recorded by the tail cuff method at $0,2,4,6,8,24$ and 48 h post-administration. Plasmatic ACE activity was determined in SHR administered water or Hpp1 $55 \mathrm{mg} / \mathrm{kg}$ at $6 \mathrm{~h}$ post-administration. BP of normotensive Wistar-Kyoto (WKY) rats administered water or $55 \mathrm{mg} / \mathrm{kg} \mathrm{Hpp} 11$ was recorded.

Results: A dose of $25 \mathrm{mg} / \mathrm{kg}$ failed to decrease the SBP in SHR ( $p>0.05$ compared to water). 55 and $85 \mathrm{mg} / \mathrm{kg}$ doses showed a significant antihypertensive effect $(\mathrm{p}<0.05)$, the maximum SBP decrease being $6 \mathrm{~h}$ post-administration $(-26.33 \pm 2.1$ and -30.45 $\pm 1.65 \mathrm{mmHg}$, respectively) compared to water. SBP initial values were recovered 24 or $8 \mathrm{~h}$ post-administration at 55 and $85 \mathrm{mg} /$ $\mathrm{kg}$, respectively, $55 \mathrm{mg} / \mathrm{kg}$ being the most effective dose. In addition, a significant inhibition of plasma ACE in SHR administered Hpp11 at $55 \mathrm{mg} / \mathrm{kg}$ was found $(\mathrm{p}<0.01)$, indicating that ACE in- hibition is the involved mechanism in the antihypertensive effect. Finally, an undesirable hypotensive effect of Hpp11 was ruled out at $55 \mathrm{mg} / \mathrm{kg}$ in WKY, showing that BP decrease is linked to a previously existing pathological state of hypertension.

Conclusions: These results suggest that Hpp11 could be used as a functional ingredient with antihypertensive effect, although clinical studies are needed to demonstrate its long-term antihypertensive efficiency in humans.

References: ${ }^{1}$ F. I. Bravo, L. Arola; B. Muguerza. "Method for obtaining a chicken feet hydrolysate with antihypertensive activity, the hydrolysate that is obtained and the peptides it contains". ES2606954 B1. December 12, 2017.

Conflict of interest: Authors declare no conflict of interest.

Acknowledgements: This work was supported by grant numbers AGL-2013-40707-R and AGL2016-77105-R from the Spanish Government and grant number 2015PMF-PIPF-51 from URVMartí Franqués.

Key words: chicken feet hydrolysate / ACE inhibitory activity / antihypertensive effect / spontaneously hypertensive rats / wistar-kyoto rats.

\section{5 \\ CHERRY CONSUMPTIONTRIGGERS A DIFFEREN- TIAL METABOLIC RESPONSE IN NORMOWEIGHT AND OBESE FISCHER 344 RATS EXPOSED TO SHORT AND LONG PHOTOPERIODS}

\author{
${ }^{(1)}$ R. Mariné-Casadó; (2)C. Domenech-Coca; (1)J.M. Del Bas; ${ }^{(2) C}$. Bladé; \\ ${ }^{(1,2)}{ }^{2}$ L. Arola; ${ }^{(1)}$ A. Caimari. \\ (1)Nutrition and Health Unit. Eurecat-Centre Tecnològic de \\ Catalunya. Reus. Spain; ${ }^{(2)}$ Nutrigenomics Research Group. \\ Departament de Bioquímica i Biotecnologia. Universitat Rovira i \\ Virgili. Tarragona. Spain
}

Introduction: In a previous study, we demonstrated that the chronic exposure to both short (L6, 6h/18h of light/darkness) and long (L18, 18h/6h of light/darkness) photoperiods produced profound changes in lipid and glucose metabolisms in the photoperiod-sensitive rat strain Fischer 344 (F344).

Objectives: To evaluate whether the metabolic adaptations that occur in response to a chronic day length exposure can be modified by the consumption of fruits rich in polyphenols.

Methods: Firstly, F344 male rats were fed with a standard diet, exposed for 14 weeks to a short (L6) or a long day photoperiod (L18), and orally treated with lyophilized cherry (100 mg. $\mathrm{kg}^{-1}$ body weight/day) or a vehicle during the last 10 weeks. In a second study, F344 rats were fed a cafeteria diet and submitted to the same photoperiods for 11 weeks and supplemented with cherry or vehicle during the last 7 weeks.

Results: At the end of the study, compared to the non-treated L6 counterparts, cherry-supplemented L6 normoweight rats displayed elevated gene expression levels of Cd36 and Had in the soleus muscle, and decreased phosphorylated levels of Akt2 and increased phosphorylated levels of AMPK (pAMPK) in both so- 
leus and gastrocnemius muscles. In L6-obese rats, cherry administration increased the circulating glucose and insulin levels, and enhanced whole-body fat oxidation, which could be partially attributed to decreased gastrocnemius Glut4 mRNA levels and increased soleus pAMPK protein levels, respectively. In both studies, most of these effects were attenuated when cherry was consumed by L18 animals.

Conclusions: The intake of cherry alters lipid and glucose metabolisms mainly in normoweight and obese animals chronically exposed to the short photoperiod, which suggest that the effects triggered by this fruit are highly influenced by the circannual endogenous metabolism. The pathophysiological significance of these results requires further investigation.

Conflict of interest: Authors declare no conflict of interest.

Key words: photoperiod / polyphenols / cherry / metabolic homeostasis.

\title{
076
}

\section{INFLUENCE OF THE NATURE OF EMULSIFIERS ON THE BIOACCESSIBILITY OF ß-CAROTENE EN- CAPSULATED IN NANOEMULSIONS}

\author{
(1)J. Teixé; ${ }^{(1)}$ G. Oms; ${ }^{(2)}$ K. Najman; ${ }^{(1)}$ I. Odriozola. \\ (1)Department of Food Technology. University of Lleida. Lleida. \\ Spain; ${ }^{(2)}$ Department of Functional Food and Commodities. \\ Faculty of Human Nutrition and Consumption. Warsaw \\ University of Life Sciences (SGGW). Warsaw. Poland.
}

Introduction: Interest in bioactive compounds such as $\beta$-carotene is currently growing due to their health benefits. However, these compounds are degraded during the digestion process and as a result are poorly absorbed in the human intestine. Nanoemulsions have been used as systems to encapsulate and protect these lipophilic active compounds in the gastrointestinal tract in order to improve their functionality. Nevertheless, the design of these systems is still a challenge.

Objectives: To obtain oil-in-water nanoemulsions containing $\beta$-carotene using lecithin as a natural emulsifier or Tween 20 as a synthetic emulsifier, at different concentrations (2\%, $4 \%$, and $8 \%)$.

Methods: High-pressure microfluidization was used to produce nanoemulsions by reducing the particle size to nanometrical range. The effects on particle size, electrical charge and $\beta$-carotene bioaccessibility were compared with conventional homogenization.

Results: As expected, nanoemulsions presented smaller particle size (150-250 $\mathrm{nm})$ than conventional emulsions (9-18 $\mu \mathrm{m})$. On the other hand, it was found that the higher the surfactant concentration, the smaller the particle size, irrespective of the surfactant used. The highest electrical charges were observed (between - 55 $\mathrm{mV}$ and $-90 \mathrm{mV}$ ) when using lecithin, indicating that this emulsifier ameliorated the stability of the systems. The bioaccessibility of $\beta$-carotene improved significantly in nanoemulsions compared to conventional emulsions. The enhanced bioaccessibility, up to $40 \%$, observed in nanoemulsions stabilized with lecithin at $8 \%$ may be related to a better $\beta$-carotene solubilisation in the mixed micelles.

Conclusions: In conclusion, nanoemulsions could be helpful to improve $\beta$-carotene bioaccessibility, lecithin being a better option than the synthetic emulsifier in the formulation of these systems.

Conflict of interest: Authors declare no conflict of interest. Key words: nanoemulsions / beta-carotene / bioaccessibility / surfactants. 


\section{Poster Abstract Presentations}

\section{7 \\ BIOACTIVE COMPOUNDS AND MICROENCAP- SULATION EFFICIENCY OF BLUEBERRY MICRO- CAPSULES}

\author{
${ }^{(1)}$ A. Olivares La Madrid; ${ }^{(1)}$ M. Armada; ${ }^{(2)}$ A. Cravero; ${ }^{(1)}$ F. Villalva. \\ (1)Universidad Nacional de Salta. INIQUI. Salta. Argentina; \\ (2)Universidad Nacional de Salta. Facultad de Ciencias de la Salud. \\ Salta. Argentina.
}

Introduction: Blueberries are rich in bioactive compounds (BAC) that prevent or delay the onset of degenerative diseases. Their short shelf life limits their availability, which has prompted the application of technological preservation processes such as spray drying.

Objectives: To determine the content of BAC and the microencapsulation efficiency (EE) of microcapsules (MC) obtained by spray.

Methods: Emerald blueberries from Extraberries S.A (Metán-Salta-Argentina) were stored in hermetic bags at $18^{\circ} \mathrm{C}$ until used. Fruits were thawed and processed, the pulp was sieved (60mesh), and the juice was mixed with different barrier materials: maltodextrin (MD) 30\%; arabic gum (AG) 30\%; modified starch (MS) $30 \%$ and mixtures MD15\%+AG15\%; MD15\%+MS15\%; MD10\%+AG10\%+MS10\%; and AG15\%+MS15\%. Seven MC were obtained for the determination of EE (Idham et al, 2012) and BAC: anthocyanins (A) (Giusty-Wrostald,2000); proanthocyanidins (PAC) by Vanillin/HCl (Price et al.,1978); polyphenols (PF) by FolinCiocalteu (Sinlgeton \& Rossi,1965) and antioxidants (AT) by ABTS (Re, et al 1999). BAC were determined in extracts with acidified alcohol, at $30^{\circ} \mathrm{C} \pm 5$ for 30 minutes.

Results: EE was $86.27 \%$ MS; $85.45 \%$ MD; $82.35 \%$ $\mathrm{MD}+\mathrm{AG}+\mathrm{MS} ; 81.42 \%$ AG; $74.65 \% \mathrm{MD}+\mathrm{MS} ; 74.5 \% \mathrm{AG}+\mathrm{MS}$ and $67.1 \%$ in MD+AG. A were: $3414 \mathrm{mg} \mathrm{AG+MS;} \mathrm{2509mg} \mathrm{MD;}$ $2091.67 \mathrm{mg} \mathrm{MD}+\mathrm{MS} ; 1586 \mathrm{mg} \mathrm{MS} ; 1097.93 \mathrm{mg} \mathrm{MD}+\mathrm{AG}+\mathrm{MS}$; 1020.93mg MD+AG and AG 789mg cyanidin3glucoside/100gr, respectively. PAC were: $1879.35 \mathrm{mg}, 1860.20 \mathrm{mg}, 1810.42 \mathrm{mg}$, $1169.64 \mathrm{mg}, 976 \mathrm{mg}, 621.34 \mathrm{mg}, 598.21 \mathrm{mg}$ catechin in AG+MS; $\mathrm{MD}+\mathrm{MS} ; \mathrm{MD}+\mathrm{MS} ; \mathrm{MD}+\mathrm{AG} ; \mathrm{MD}+\mathrm{AG}+\mathrm{MS}$ and $\mathrm{AG}$, respectively. $\mathrm{PF}$ in $\mathrm{MC}$ were in $\mathrm{MD}+\mathrm{AG} 872.76 \mathrm{mg}$; MD 770.31 mg, $\mathrm{AG}+\mathrm{MS}$ $758.43 \mathrm{mg}, \mathrm{MD}+\mathrm{MS} 643.37 \mathrm{mg}, \mathrm{MD}+\mathrm{AG}+\mathrm{MS} 586.67 \mathrm{mg}, \mathrm{AG}$ $475 \mathrm{mg}$ and $\mathrm{MS} 265.61 \mathrm{mg}$ EAG. AT in MC were: in MD+MS+AG 741.01ul, MD+MS 731.34ul, MD+AG 664.25ul, MD 541, 87ul, MS 512.35ul, AG+MS 499.47ul and in AG 478, 54ulTrolox /100gr.

Conclusions: Spray drying preserves BAC. The variability in content can be attributed to the coat material used.

Conflict of interest: Authors declare no conflict of interest.

Key words: blueberry / microencapsulation / bioactive compounds.

\section{8 \\ BIOACTIVE COMPOUNDS IN QUINOA, AMA- RANTH AND TARWI}

\author{
${ }^{(1)}$ A. Olivares La Madrid; ${ }^{(1)}$ F. Villalva, F; ${ }^{(2)}$ A. Cravero; ${ }^{(1)}$ M. Armada. \\ (1)Universidad Nacional de Salta. INIQUI. Salta. Argentina; \\ (2) Universidad Nacional de Salta. Facultad de Ciencias de la Salud. \\ Salta. Argentina.
}

Introduction: Andean grains are foods of high nutritional value, containing phytochemicals such as polyphenols with antioxidant power, which exert an effect against degenerative diseases.

Objectives: To study the content of bioactive compounds (BAC) in quinoa, amaranth and tarwi.

Methods: For BAC extraction, the whole grains were milled in a mill for 5 minutes. The products obtained, quinoa flour (QF), amaranth flour (AF) and tarwi flour (TF), were stored in closed containers until their analysis. In $\mathrm{QF}, \mathrm{AH}$ and $\mathrm{TH}$, polyphenols (PF) were determined by the Folin Ciocalteu method (Singleton Rossi, 1978), in a calibration curve with gallic acid, the content being expressed as mg acid gallic equivalent (EAG). The antioxidant content was quantified with the Radical ABTS method (Re, et al. 1999), on a calibration curve with Trolox, and the results were expressed as $\mu \mathrm{M}$ Trolox equivalents (ET). For the readings of the water-soluble compounds, an extract with water was prepared, and the fat-soluble extracts were extracted with a mixture of hexane-acetic acid-acetone.

Results: The content of soluble PF was $60.59 \mathrm{mg}$ in QF, 39.07 in $\mathrm{TF}$ and $14.59 \mathrm{mg}$ EAG / $100 \mathrm{~g}$ in AF. The fat-soluble fraction was $1024.05 \mathrm{mg}$ in TF, 513.70mg in QF and 124.20mg EAG / 100g in AF. The antioxidants in the water-soluble extract varied in the range of $297.65 \mu \mathrm{M}$ in QF, $274.57 \mu \mathrm{M}$ in TF and in $\mathrm{AF} 105.76 \mu \mathrm{M}$ ET / 100g; liposoluble antioxidants were $465.65 \mu \mathrm{M}$ in QF, $461.36 \mu \mathrm{M}$ in TF and in AF 276.18 $\mu \mathrm{M}$ ET / 100g.

Conclusions: Andean grains are a useful source of BAC. The variability between samples can be attributed to the chemical structure of the grains.

Conflict of interest: Authors declare no conflict of interest.

Key words: bioactive compounds / quinoa / amaranth / tarwi.

\section{9}

\section{EVALUATION OF ACTIVE COMPONENTS OF CA- LATHEA LUTEA AS A COATING IN FOOD PROD- UCTS}

\begin{abstract}
${ }^{(1)}$ R. Olivero; ${ }^{(1)}$ A. Florez; ${ }^{(2)}$ Y. Aguas.
(1)Programa de Ingeniería Agroindustrial. Grupo de Investigación en Bioprocesos. Universidad del Atlántico. Barranquilla Atlántico. Colombia; (2)Programa de Ingeniería Agroindustrial. Universidad de Sucre. Sucre. Colombia.
\end{abstract}

Introduction: In Colombia, bijao leaves (Calathea lutea) are used as wrappers for some foods, but their bioactive properties 
are unknown. Other applications in the food industry that give added value to this undervalued and little-studied leaf in Colombia are unknown. It is of great importance to evaluate the active components of $\mathrm{C}$. lutea as a softener and flavoring, with the aim of replacing some of the commercial synthetic meat softeners.

Objectives: To evaluate the bioactive components of C. lutea leaves as a coating in food products.

Methods: Several treatments were compared in order to assess the effect of the bijao leaf on the physicochemical $(\mathrm{pH}$, water retention capacity) and organoleptic characteristics of beef, including hardness and texture, which were evaluated at 72 and 96 hours. The results were analyzed with a multiple-rank test of the SPSS Statistics 2.0 program.

Results: Meat treated with C. lutea leaves was less hard compared with the other treatments. There was a significant difference in the firmness of meat between the types of treatment. There were no significant differences between the results obtained in CRA. The meat samples studied were of the PSE type (pale, soft and exudative), with a $\mathrm{pH}$ below 5.5. The $\mathrm{pH}$ did not show any significant changes in any of the treated samples.

Conclusions: The best softening effect on meat according to the texture profile and tenderness analysis was observed in the sample of meat treated with Calathea lutea. The treatments carried out did not directly influence the water retention capacity of the meat.

Conflict of interest: Authors declare no conflict of interest.

Key words: treatments / cra /texture / firmness / meat.

\section{0 \\ BIOACTIVE COMPONENTS IN THE AVOCADO SEED (PERSEA AMERICANA) AS AN INHIBITOR OF ENZYMATIC BROWNING.}

\author{
${ }^{(1)}$ R. Olivero; ${ }^{(1)}$ A. Florez; ${ }^{(2)}$ Y. Aguas. \\ (1) Programa de Ingeniería Agroindustrial. Grupo de Investigación \\ en Bioprocesos. Universidad del Atlántico. Barranquilla Atlántico. \\ Colombia; (2)Programa de Ingeniería Agroindustrial. Universidad \\ de Sucre. Sucre. Colombia.
}

Introduction: The color changes produced by enzymatic browning is a disadvantage in the sale of fruit pulp and negatively impresses the consumer due to associations between color and nutritional and sensory qualities. Enzymatic browning can be controlled by chemical and physical methods, often used in combination. New sources of enzymatic browning inhibitors are being studied, in this case the avocado fruit seed, which was evaluated as a promising antioxidant.

Objectives: To evaluate the bioactive compounds in the avocado seed (Persea americana) as an inhibitor of enzymatic browning.

Methods: The effect of the avocado seed as an inhibitor of browning was assessed on avocado pulp. Treatments with fresh seed and lyophilized seed were carried out. For comparison purposes, a sample of pulp without any treatment and a sample of avocado pulp treated with 3000 ppm of citric acid was analyzed in

XVII Conference of SEÑ and X Meeting of ACCA each of the conditions. The software used was Adobe ${ }^{\circledast}$ Photoshop ${ }^{\star}$ CS3 Extended (Adobe Systems Incorporated, USA) including the PANTONE ${ }^{\circledast}$ color guides (X-Rite, Inc., Grand Rapids, MI, USA), with regulators in the color space CIE- $L^{*} a^{*} b^{*}$.

Results: The treatment with fresh seed maintained the values closest to the initial value of $L^{*}$, that is, those with the smallest degree of obscuration based on the ranges of the luminosity of CIE LAB.

Conclusions: The lyophilized avocado seed (Persea americana), variety Lorena, produced a slight decrease in the evolution of the oxidation reaction caused by polyphenoloxidase in the avocado paste.

Conflict of interest: Authors declare no conflict of interest. Key words: antioxidant / color / fruit / photoshop / pulp.

\section{1 \\ B-CRYPTOXANTHIN FROM FERMENTED OR- ANGE JUICE IS MORE BIOAVAILABLE IN HU- MANS THAN FROM ORANGE JUICE}

\author{
${ }^{(1)}$ I. Cerrillo; ${ }^{(2)} D$. Hornero-Méndez; ${ }^{(1)} A$. Ortega; ${ }^{(3)} R$. Rodríguez- \\ Griñolo; ${ }^{(1)} B$. Escudero-López; ${ }^{(1)}$ F. Martín; ${ }^{(1)}$ M.S. Fernández-Pachón. \\ (1)Área de Nutrición y Bromatología, Departamento de Biología \\ Molecular e Ingeniería Bioquímica. Universidad Pablo de \\ Olavide. Sevilla. Spain; ${ }^{(2)}$ Departamento de Fitoquímica de \\ Alimentos. Instituto de la Grasa-CSIC. Campus Universitario \\ Pablo de Olavide. Sevilla. Spain; ${ }^{(3)}$ Área de Estadística e I.O. \\ Departamento de Economía, Métodos Cuantitativos e Historia \\ Económica. Universidad Pablo de Olavide. Sevilla. Spain.
}

Introduction: Orange juice (OJ) has one of the highest carotenoid contents reported in any fruit-derived food. The consumption of $\mathrm{OJ}$ is estimated to provide a majority of the intake of $\beta$-cryptoxanthin. This pigment is a vitamin A precursor and has been found to exert multiple biological activities in the organism. Food matrix characteristics and food processing techniques influence the bioaccessibility and absorption of carotenoids, respectively.

Objectives: The aim of this study was to compare the bioavailability of carotenoids, especially $\beta$-cryptoxanthin, from OJ with that from a beverage obtained by alcoholic fermentation of orange juice (FOB).

Methods: Seven healthy subjects were recruited for the controlled intervention. After overnight fasting, baseline blood was drawn $(0 \mathrm{~h})$. Volunteers drank $500 \mathrm{~mL}$ of $\mathrm{OJ}$ and consecutive blood samples were collected at 1, 2, 3, 4, 5, 6 and $8 \mathrm{~h}$. After a two-week wash-out the intervention was repeated with the consumption of FOB. The postprandial plasmatic concentrations of $\beta$-cryptoxanthin, lutein and zeaxanthin were evaluated by HPLC.

Results: $\beta$-cryptoxanthin and lutein absorption from FOB was significantly higher than from $\mathrm{OJ}(\mathrm{P}=0.004$ and $\mathrm{P}=0.019$, respectively). In the case of zeaxanthin, differences in absorption between FOB and OJ were not statistically significant. The mean baseline-corrected area under the concentration curve (AUC0-8 h) for $\beta$-cryptoxanthin, lutein and zeaxanthin was 24.6-, 1.3- and 4.65-fold larger, respectively, after FOB versus OJ consumption. 
Conclusions: The alcoholic fermentation of OJ improved the bioavailability of $\beta$-cryptoxanthin. Regular consumption of FOB could be a good dietary source of bioavailable $\beta$-cryptoxanthin and exert healthy effects derived from the multiple biological activities of this bioactive compound. This novel food can be considered a functional beverage.

Conflict of interest: Authors declare no conflict of interest.

Key words: $\beta$-cryptoxanthin / carotenoids / bioavailability / orange / fermentation.

\section{2 \\ THE DAILY INTAKE OF ARBEQUINA TABLE OL- IVES REDUCES THE PRENEOPLASTIC MORPHO- LOGICAL MARKERS OF COLON CANCER IN- DUCED BY 1,2-DIMETHYLHYDRAZYNE IN RATS}

\author{
R. Moreno-González; M.E. Juan; J.M. Plana.
}

Departament de Bioquímica i Fisiologia. Institut de Recerca en Nutrició i Seguretat Alimentària (INSA-UB). Universitat de Barcelona (UB). Barcelona. Spain.

Introduction: Table olives constitute an important edible fruit of the Mediterranean diet and a source of bioactive compounds with protective activities against several types of tumors, including colon cancer.

Objectives: To investigate the effect of table olives in early stages of colon carcinogenesis induced by 1,2-dimethylhydrazine (DMH).

Methods: Sprague-Dawley rats were distributed in a DMHgroup that received 3- weekly injections of the carcinogen and a control-group given only the solvent. Animals were further divided in two subgroups administered orally by gavage with either water or olives for 49 days. Arbequina table olives were prepared as a ground suspension at a dose of $4 \mathrm{~g} / \mathrm{kg}$ (equivalent to the ingestion of 30 olives by a 70-kg-human). Rats were killed, and the colon was excised and fixed with formalin. Aberrant crypt foci (ACF) were counted after staining with methylene blue, while the highiron diamine Alcian blue staining was used to evaluate mucin-depleted foci (MDF).

Results: No preneoplastic lesions were found in the control groups administered water or olives. $\mathrm{DMH}$-injected rats developed ACF following a regionalized pattern with $12 \pm 1,34 \pm 4$ and $68 \pm 11$ in the proximal, medial and distal colon, respectively. However, consumption of olives reduced ACF by $44 \%, 46 \%$ and $60 \%$ in the same segments. Moreover, olives decreased ACF with 1, 2, 3 and $\geq 4$ crypts in the total colon by $52 \%, 56 \%, 63 \%$ and $38 \%$, respectively. MDF were not detected in the proximal segments of either DMH group. Olive intake diminished the count of the dysplastic MDF lesions by $21 \%$ and $44 \%$ in the medial and distal segments, respectively.

Conclusions: The reduction of the preneoplastic lesions induced by $\mathrm{DMH}$ in rat colon by table olives suggests that the daily consumption of the fruit of Olea europaea L. elicits a cancer chemopreventive activity.
Acknowledgements: Supported by AGL2013-41188 (MINECO) and 2017SGR945 (Generalitat de Catalunya).

Conflict of interest: Authors declare no conflict of interest. Key words: colon / cancer / olives / rat.

\section{3 \\ CAROB POD AND WAKAME SNACK FORMULA- TIONS PROMOTED HEPATIC FATTY OXIDATION}

\author{
${ }^{(1,2)}$ L. Aguirre; ${ }^{(3)} D$ Rico; ${ }^{(4)} C$. Martínez-Villaluenga; ${ }^{(1,2)}$ l. Milton- \\ Laskibar; ${ }^{(5)}$ D.A. De Luis; ${ }^{(1)}$ J. Sangrador; ${ }^{(1,2)}$ M.T. Macarulla; ${ }^{\left({ }^{(3)}\right.}$ A.B \\ Martin-Diana. \\ (1) University of the Basque Country (UPV/EHU). Lucio Lascaray \\ Research Institute. Vitoria. Spain; ${ }^{(2)} \mathrm{CIBERobn}$. Institute of Health \\ Carlos III. Spain; ${ }^{(3)}$ Agro-Technological Institute of Castilla y \\ León (ITACyL). Spain; ${ }^{(4)}$ Institute of Food Science, Technology \\ and Nutrition (ICTAN-CSIC). Madrid. Spain; ${ }^{(5)}$ Hospital Clínico \\ Universitario. University of Valladolid. Spain.
}

Introduction: The metabolic syndrome, which represents a collection of abnormalities including obesity, insulin resistance, dyslipidemia, fatty liver, and a proinflammatory state, has become a major health concern worldwide. Results from our laboratory demonstrated the effectiveness of Ceratonia siliqua L. (carob fruit) and Undaria pinnatifida (wakame seaweed) snacks in diminishing hepatic triacylglycerol (TG) content in rats with metabolic syndrome (MetS).

Objectives: To determine whether fatty acid oxidation is a mechanism of action that explains the observed improvement in fatty liver in rats treated with functional snack formulations.

Methods: Forty adult male Wistar rats were fed with a commercial high-fat high-fructose diet for 8 weeks in order to generate the MetS. After this period, ten animals were sacrificed (MetS group) and the remaining animals were divided into 3 groups. All groups were fed with a semi-purified standard diet for 4 additional weeks. The groups were the control group (C) and two snack groups where all the complex carbohydrates of the diet were replaced by a snack formulation with combinations of 1/50 (1/50 group) or $1 / 5$ (1/5 group) of wakame/carob pod. Carnitine palmitoyltransferase la (CPT-1a) and citrate synthase (CS) activities were measured spectrophotometrically in the liver.

Results: Snack supplementation elevated CPT-1a activity, but only $1 / 50$ snack formulations reached statistical significance $(+181 \%$ vs MetS group). CS activity was raised in both snack groups $(+148 \%$ for $1 / 5$ group and $+186 \%$ for $1 / 50$ group vs MetS group). No changes were observed in CPT-1a and CS activities when comparing control and MetS groups.

Conclusions: Fatty acid flux to mitochondria as well as mitochondria activity promotion demonstrate that fatty acid oxidation can be one of the mechanisms of action for fatty liver improvement by carob pod and wakame snack formulations.

Acknowledgements: This study has been supported by INIA: RTA2014-0037-C02.

Conflict of interest: Authors declare no conflict of interest.

Key words: metabolic syndrome / liver / carob / wakame. 


\section{4}

\section{DELIPIDATING MECHANISM OF ACTION IN 3T3-L1 MATURE ADIPOCYTES TREATED WITH A COMBINATION OF CAROB POD AND WAKAME}

\author{
${ }^{(1,2)}$ J. Miranda; ${ }^{(3)} D$. Rico; ${ }^{(1,2)}$ A. Lasa; ${ }^{(4)} C$. Martínez-Villaluenga; ${ }^{(3)} A . B$. \\ Martin-Diana; ${ }^{(1)}$ M. Arana; ${ }^{(4)}$ E. Peñas.
}

(1)University of the Basque Country (UPV/EHU). Lucio Lascaray Research Institute. Vitoria. Spain; ${ }^{(2)}$ CIBERobn. Institute of Health Carlos III. Spain; ${ }^{\left({ }^{3}\right)}$ Agro-Technological Institute of Castilla y León (ITACyL). Valladolid. Spain; ${ }^{(4)}$ Institute of Food Science, Technology and Nutrition (ICTAN-CSIC). Madrid. Spain.

Introduction: Scientific research is constantly looking for new molecules that can be used as effective functional biomolecules in the fight against obesity and its co-morbidities. Our research team has previously reported the potential of Ceratonia siliqua L. (carob fruit) and Undaria pinnatifida (wakame seaweed) extracts in reducing triacylglycerol accumulation in mature adipocytes.

Objectives: To analyze the effects of extracts from carob pod, wakame, and their combination on triacylglycerol (TG) accumulation in 3T3-L1 mature adipocytes, and to explore the mechanisms of action of this combination.

Methods: Confluent 3T3-L1 pre-adipocytes were differentiated to mature by an induction media containing insulin, dexamethasone and 3-isobutyl-1-methylxanthine. After 10 days of differentiation, cells were treated with methanolic extracts of carob pod $(50 \mathrm{mg} / \mathrm{mL})$, wakame $(1 \mathrm{mg} / \mathrm{mL})$ and their combination. The maturation state for treatment was selected after performing a gene expression time course during the differentiation process. Cells were harvested 24 hours after the treatment and TG content was quantified. Total cell RNA was extracted with Trizol and ATGL (adipose triglyceride lipase), and HSL (hormone-sensitive lipase), ACC (acetyl-CoA carboxylase), FAS (fatty acid synthase), BCL-2 (B-cell lymphoma 2), BAX (Bcl-2-associated X protein), and gene expression was measured by real time polymerase chain reaction.

Results: Wakame extract and its combination with carob pod decreased TG. Wakame reduced the HSL gene expression and increased BCL-2. In contrast, the combination diminished the FAS gene and raised BAX gene expression. No changes were observed in the remaining analyzed genes.

Conclusions: The decrease in mature adipocyte triacylglycerol content induced by the combination of carob pod and wakame, but not by wakame alone, may be due, at least in part, to lipogenesis inhibition and apoptosis activation. Consequently, although further research is needed, this combination may be proposed as a potential fat-reduction inducer ingredient.

Acknowledgements: Study supported by INIA: RTA20140037-C02.

Conflict of interest: Authors declare no conflict of interest.

Key words: adipocyte / lipogenesis / apoptosis / carob / wakame.

\section{5 \\ QUANTITATIVE METHOD TO DETERMINE OLEO- CANTHAL AND OLEACEIN IN VIRGIN OLIVE OILS BY 1H NMR}

\author{
${ }^{(1,2)}$ A. López-Yerena; ${ }^{(1,2)}$ J. Lozano-Castellón; ${ }^{(1,2)}$ A. Vallverdú- \\ Queralt; ${ }^{(3)}$ M. Pérez Bosch; ${ }^{(1,2)}$ P. Quifer Rada; ${ }^{(4)} B$. Jiménez; ${ }^{(2)}$ E. \\ Escribano; ${ }^{(1,2)}$ R.M. Lamuela-Raventós.
}

\begin{abstract}
(1)Department of Nutrition, Food Sciences and Gastronomy. XaRTA. Institute of Nutrition and Food Safety (INSA.UB). Faculty of Pharmacy and Food Sciences. University of Barcelona. Barcelona. Spain. ${ }^{(2)}$ CIBEROBN. Instituto de Salud Carlos III. Spain. (3)Laboratory of Organic Chemistry. Department of Nutrition, Food Sciences and Gastronomy. XaRTA. Institute of Nutrition and Food Safety (INSA.UB). Faculty of Pharmacy and Food Sciences. University of Barcelona. Barcelona. Spain. ${ }^{(4)}$ Ministry of Agriculture and Fisheries. Agricultural Research Training Centre. Cordoba. Spain. ${ }^{(5)}$ CIBER Fisiopatología de la Obesidad y la Nutrición (CIBERobn), Instituto de Salud Carlos III, Dept of Pharmacy and Pharmaceutical Technology, Faculty of Pharmacy and Food Sciences.
\end{abstract}

Introduction: Virgin olive oil (VOO) is one of the essential components of the Mediterranean diet. The most important bioactive components in VOO are phenolic compounds, which have been associated with a reduced risk of suffering cardiovascular diseases and a protective effect against cancer. Two key secoiridoids present in VOO, oleocanthal and oleacein, are gaining clinical and nutritional interest due to their proven biological properties. Oleocanthal is a promising therapeutic agent for the treatment of inflammatory degenerative joint diseases and oleacein has displayed significant antibreast cancer properties.

Objectives: The aim of this research was to determine the effect of ecological and conventional cropping systems on the content of oleocanthal and oleacein in VOO made from Picual and Hojiblanca varieties and its rapid quantification by $1 \mathrm{H}$ NMR.

Methods: A method developed for the measurement of oleocanthal and oleacein levels by quantitative $1 \mathrm{H}$ NMR was applied in Picual and Hojiblanca VOO samples. The VOO was produced from olive trees in a two-section plot under ecological and conventional conditions without irrigation.

Results: The signals of the aldehydic protons at 9.632 and 9.646 ppm were assigned to oleocanthal and oleacein, respectively. The results showed that the two secoiridoids were present in ecological and conventional VOO in different amounts, with the highest concentrations recorded in ecological VOO samples.

Conclusions: The growing conditions (conventional versus ecological) affected the levels of oleacein and oleocanthal in VOO. The overall evidence points to an enhancement of phytochemical content in organically grown fruits. This growth system causes stress for the plants, leading to an increase in the level of natural defences such as secondary metabolites, which possess significant biological activities related to antioxidant and anti-inflammatory activity.

Conflict of interest: Authors declare no conflict of interest.

Key words: oleocanthal / oleacein / 1H NMR. 


\section{6}

\section{EFFECTS OF DIET SUPPLEMENTATION ON CERE- BRAL POLYUNSATURATED FATTY ACID PROFILE AND PROTEIN OXIDATIVE MODIFICATION IN A PORCINE MODEL OF OBESITY}

\author{
${ }^{(1)}$ R. Berdún; ${ }^{(1)} M$. Jové Font; ${ }^{(2)}$ M. Font-i-Furnols; ${ }^{(3)}$ J. Moreno; ${ }^{(3)} M$. \\ Rodriguez-Palmero; ${ }^{(4)} D$. Valent; ${ }^{(2)}$ J. Tibau; ${ }^{(1)}$ M. Portero-Otin.

\begin{abstract}
(1)NUTREN-Nutrigenomics. IRBLleida-UdL. Lleida. Spain; ${ }^{(2)}$ IRTAAnimal Breeding and Genetics and Product Quality Programs. Barcelona. Spain; ${ }^{(4)}$ Departament de Bioquímica i Biologia Molecular. Facultat de Veterinària. Universitat Autònoma de Barcelona. Cerdanyola del Vallès. Spain.
\end{abstract} \\ Monells. Spain; ${ }^{(3)}$ Research Department. Laboratorios ORDESA.
}

Introduction: Supplementation with omega-3 fatty acids during childhood and puberty has been suggested as a strategy to prevent metabolic disorders associated with obesity.

Objectives: To study the effects of a western-type diet supplemented with bioactive ingredients compared to a standard diet in a porcine model of prepuberal obesity on fatty acid composition as well as several biomarkers of oxidative damage.

Methods: At the IRTA Pig Experimental Station, forty-two female littermates of Duroc line were fed ad libitum 4 different diets from 60 to 130 days of age: a standard diet (SD1); western diet (WD2); western diet (WD3) with $50 \%$ of protein as rice protein hydrolysate and the probiotic B. breve (5x1010 ufc/day); and a western diet (WD4) with the same composition as WD3 but containing $2 \%$ omega- 3 fatty acids. At slaughter, plasma and brain samples were obtained and the fatty acid profile was analysed by derivatization and gas chromatography. In addition, biomarkers of protein oxidative damage were measured by mass spectrometry.

Results: Weight gain in WD2-fed animals was higher $(\mathrm{p}<0.01)$ than in the SD1-fed group. Interestingly, despite similar calorie intake, weight gain was significantly attenuated in WD3 and WD4 $(\mathrm{p}<0.007$ after ANOVA adjusted for $\mathrm{FDR}=0.01)$. In relation to fatty acid status, WD4 showed higher a-linolenic and docosahexaenoic acid content in comparison with WD2 and WD3, both in plasma and brain $(\mathrm{p}<0.05)$. In addition, the plasma lipid peroxidation index was lower, and plasma and brain anti-inflammatory indexes were higher in the WD4 group. Regarding oxidative biomarkers, supplementation with bioactive ingredients decreased brain content of 5-hydroxy-2-amino-valeric acid (HAVA) and reversed the increase in malondialdehyde-lysine (MDA-Lys) caused by the WD2 diet.

Conclusions: In a prepuberal porcine model of obesity, a combination of rice protein hydrolysate, the probiotic B. breve and omega-3 fatty acids prevented excessive weight gain, improved omega-3 nutritional status, enhanced its nervous system bioavailability and decreased several biomarkers related with oxidative modification.

Conflict of interest: José A. Moreno and María Rodriguez-Palmero are employees of the company Laboratorios Ordesa. This study was funded by Laboratorios Ordesa and CDTI.

Key words: omega-3 fatty acids / probiotic / swine.

\section{7 \\ EFFECT OF PROCESSING AND STORAGE ON THE FUNCTIONAL CAPACITY OF AN EXTRUDED CORN SNACK WITH PEACH PALM FRUIT FLOUR (BACTRIS GASIPAES HBK)}

\author{
${ }^{(1)}$ C. Agudelo; ${ }^{(2)}$ S.P. Godoy; ${ }^{(1)}$ E. García-Martínez. \\ (1)Universitat Politècnica de València. Departamento de \\ Tecnología de Alimentos. Grupo de Investigación e Innovación \\ Alimentaria. Valencia. Spain; ${ }^{(2)}$ Universidad del Cauca. Facultad \\ de Ciencias Agropecuarias. Grupo de investigación METANOIA. \\ Cauca. Colombia.
}

Introduction: The use of tropical fruits as raw materials for the production of extruded snack product opens a world of possibilities for a food industry in search of increasingly healthy products, due their particularly high content of bioactive substances, together with their specific sensory characteristics. However, the impact of the extrusion process on these components needs to be studied. Although the food safety of extruded products should be guaranteed due to their low water content, they can have problems associated with their high hygroscopicity, which can lead to chemical and biochemical deterioration during storage.

Objectives: To obtain an extruded corn snack with peach palm fruit flour (Bactris gasipaes H.B.K) as an alternative to traditional extruded cereals with the aim of improving its functional properties. To evaluate the stability of the bioactive compounds and antioxidant capacity of the snack during storage, by studying the water sorption behavior $\left(20^{\circ} \mathrm{C}\right)$.

Methods: Corn flour was mixed with peach palm flour in a proportion of 75:25 (w/w) and the blend was extruded in a single screw extruder to obtain the snack. Moisture sorption isotherms were determined by a gravimetric method at $20^{\circ} \mathrm{C}$. For that, snacks were placed in vacuum desiccators containing saturated salt solutions with water activities (aw) from 0.113- 0.75. The peach palm and corn flours, the freshly extruded snack and the equilibrated products at each aw were characterized for vitamin C, carotenoids, total phenolics and antioxidant activity. Nine replicates were made (bioactive extractions in triplicate, analyses in triplicate). Multifactor analyses of variance were carried out at 0.05 significance level in order to evaluate the differences between the samples brought about by storage conditions: relative humidity and time.

Results: Results showed that peach palm fruit flour was rich in phenolic compounds (104 mg gallic acid/ $100 \mathrm{~g}$ dry basis (db)) and carotenoids (5.69 mg b-carotene/100 g db). The extruded snacks presented a significantly $(\mathrm{p}<0.05)$ higher content of bioactive compounds (104 mg gallic acid/ $100 \mathrm{~g} \mathrm{db} ; 5.69 \mathrm{mg}$ b-carotene/100 g $\mathrm{db}$ ) than corn flour (104 mg gallic acid/ $100 \mathrm{~g} \mathrm{db} ; 5.69 \mathrm{mg}$ b-carotene/100 $\mathrm{g} \mathrm{db})$ and therefore significantly $(\mathrm{p}<0.05)$ greater antioxidant capacity ( 0.134 and $0.084 \mathrm{mmol}$ Trolox/ $100 \mathrm{~g} \mathrm{db}$, for snack and corn flour, respectively).

Conclusions: In conclusion, incorporating palm fruit flour into extruded corn products improved the product's nutritional quality because it provided high amounts of bioactive substances. In order to ensure the functional quality preservation of these 
snacks, they should be stored in environments with relative humidity lower than $43 \%$.

Conflict of interest: Authors declare no conflict of interest.

Acknowledgments: The authors thank the ADSIDEO - COOPERATION 2016 (UPV) for providing funding for the project "Promoting food safety and nutritional improvement of the rural population of the Cauca department (Colombia) through the development of sustainable native foods based on chontaduro (Bactris gasipaes, HBK)".

Key words: phenols / carotenoids / vitamin C / extrusion.

\section{8}

\section{DEVELOPMENT OF EXTRUDED CORN-BASED SNACKS WITH PEACH PALM FRUIT FLOUR (BAC- TRIS GASIPAES H.B.K): NUTRITIONAL, FUNC- TIONAL AND ANTIOXIDANT CHARACTERISTICS}

\author{
${ }^{(1)}$ C. Agudelo; ${ }^{(1)}$ A. Yuste; ${ }^{(2)}$ S.P. Godoy; ${ }^{(1)}$ E. García-Martínez.
}

(1) Universitat Politècnica de València. Departamento de Tecnología de Alimentos. Grupo de Investigación e Innovación Alimentaria. Valencia. Spain; (2)Universidad del Cauca. Facultad de Ciencias Agropecuarias. Grupo de investigación METANOIA. Cauca. Colombia.

Introduction: The development of functional corn extruded snacks by adding nutritionally rich fruit flours is an interesting strategy to promote the consumption of antioxidant-rich ingredients. Peach palm fruit (Bactris gasipaes H.B.K) is a tropical fruit especially rich in fat, carbohydrates and antioxidant compounds such as carotenoids and polyphenols. Bioactive compounds are important for human health and nutrition, especially to prevent degenerative illness, coronary and cardiovascular diseases, etc. Since peach palm fruit is a seasonal crop, its availability during the year is very limited, and it would be desirable to apply processes to extend its consumption, and at the same time preserve the nutritional and functional properties of this exceptional fruit. Extrusion is an alternative preservation process that may expand the range of products based on this fruit available on the market.

Objectives: To obtain extruded corn products with peach palm flour with high nutritional and functional properties.

Methods: Peach palm flour was added to corn flour in proportions of 0 (control), 25 and 50\%. The extrusion process was carried out in a single screw extruder (temperature profile: 40-73174- $178{ }^{\circ} \mathrm{C}$, pressure: 133.9 bar, screw speed: $150 \mathrm{rpm}$, feed rate: 20 $\mathrm{rpm})$. The flours and the obtained products were analysed in terms of water content, ash, proteins, carbohydrates, fat, main bioactive compounds (total phenols, carotenoids) and antioxidant activity (DPPH assay). Simple analyses of variance were carried out at 0.05 significance level in order to evaluate the differences between the samples.

Results: In general, the extruded products obtained stood out for their protein $(6 \mathrm{~g} / 100 \mathrm{~g}$, dry basis $(\mathrm{db})$, fat $(0.2-3 \mathrm{~g} / 100 \mathrm{~g}$ $\mathrm{db})$ and carbohydrate content $(86 \mathrm{~g} / 100 \mathrm{~g} \mathrm{db})$. The formulation with the highest proportion of peach palm fruit showed a signifi- cantly $(\mathrm{p}<0.05)$ higher value of fat $(2.9 \mathrm{~g} / 100 \mathrm{~g} \mathrm{db})$, phenolics $(60$ $\mathrm{mg}$ gallic acid/ $100 \mathrm{~g} \mathrm{db})$ and antioxidant capacity $(0.134 \mathrm{mmol}$ Trolox/100 $\mathrm{g} \mathrm{db}$ ). The extrusion process produced the total degradation of carotenoid compounds.

Conclusions: In conclusion, partial substitution of corn flour by peach palm fruit is a good option for the development of new extruded products of high nutritional and functional value.

Conflict of interest: Authors declare no conflict of interest.

Acknowledgments: The authors thank the ADSIDEO - COOPERATION 2016 (UPV) for providing funding for the project "Promoting food safety and nutritional improvement of the rural population of the Cauca department (Colombia) through the development of sustainable native foods based on chontaduro (Bactris gasipaes, $\mathrm{HBK})$ ".

Key words: extrusion / nutritional / bioactive / snacks. 


\section{Track 4: Probiotics and prebiotics}

\section{Nutrition of Metabolism}

\author{
Reviewers: \\ Montserrat Bosch \\ Leitat - Centro Tecnológico
}

Margarita Castell Escuer

Universitat de Barcelona

Ascensión Marcos Sánchez

Consejo Superior de Investigaciones Científicas

Miquel Moretó Pedragosa

Universitat de Barcelona

\section{Oral Abstract Presentations}

\section{9}

\section{EFFECT OF SYNBIOTIC FOOD CONTAINING FRUCTO-OLIGOSACCHARIDES FROM AGAVE SALMIANA ON THE LIPID PROFILE OF MEXICAN PREPUBERTAL OVERWEIGHT CHILDREN.}

${ }^{(1)}$ O.González-Acevedo; ${ }^{(2)}$ D.P. Portales-Pérez; ${ }^{(2)}$ F. Martínez-Gutiérrez; ${ }^{(2)} M$. Vega-Cárdenas; ${ }^{(2)} D$. Vázquez-Maldonado; (2)J.M. VargasMorales; ${ }^{(3)} \mathrm{C}$. Godínez-Hernández; ${ }^{(1)} A$. Rodríguez-Lara; ${ }^{(1)} \mathrm{C}$. GomezLlorente.

\begin{abstract}
(1)Instituto de Nutrición y Tecnología de Alimentos. Centro de Investigación Biomédica. Universidad de Granada. Granada. Spain; ${ }^{(2)}$ Facultad de Ciencias Químicas. Universidad Autónoma de San Luis Potosí. San Luis Potosí. Mexico; ${ }^{(3)}$ Instituto de Zonas Desérticas. Universidad Autónoma de San Luis Potosí. San Luis Potosí. Mexico.
\end{abstract}

Introduction: The use of a synbiotic containing fructo-oligosaccharides (FOS) from Agave salmiana together with a probiotic (S. thermophilus) has been shown to reduce the cholesterol and triglyceride concentration in Wistar rats. The intestinal production of short-chain fatty acids (SCFA), carbon disulfide and methyl acetate were also modified by the effect of the synbiotic.

Objectives: To evaluate the effect of this synbiotic with Agave salmiana FOS on the lipid profile of prepubertal children in comparison with a commercial dairy food containing L. casei Shiro$\mathrm{ta}(\mathrm{LcS})$ plus S. thermophilus as a control group.

Methods: We performed a nutritional intervention for 6 weeks. Forty-five obese and overweight prepubertal children (6-11 years old) were randomly assigned into three groups: control group, experimental group $1\left(\mathrm{LcS}\right.$ and S.thermophilus $\left.10^{8}(\mathrm{CFU}) / \mathrm{g}\right)+$ inulin

\footnotetext{
KARGER XVII Conference of SEÑ and X Meeting of ACCA
}

$3 \mathrm{~g}$ and experimental group $2\left(\left(\mathrm{LcS}+\mathrm{S}\right.\right.$.thermophilus $\left.10^{8}(\mathrm{CFU}) / \mathrm{g}\right)$ + FOS of Agave salmiana 3g. Informed consent was obtained for the parents. Blood samples were taken at the beginning and the end ( $\mathrm{t} 0$ and $\mathrm{t} 1$ ) of the intervention.

Results: During the intervention period ( $\mathrm{t} 0$ versus $\mathrm{t} 1$ ) the control group showed a significant increase $(\mathrm{P}<0.001)$ of glucose and HDL-cholesterol levels, whereas the LDL-cholesterol levels decreased. Similarly, the experimental group 1 showed a significant increase in glucose, total cholesterol and HDL-cholesterol. In contrast, the experimental group 2, consisting of children fed with a synbiotic diet with FOS from A. salmiana, showed no increase in concentrations of cholesterol, LDL-cholesterol, or triglycerides.

Conclusions: The interventions with probiotic and synbiotics generated similar modifications in the metabolic profile of prepubertal children, evidenced by modifications in glucose and lipid profile. The synbiotic group with Agave salmiana was the only one that did not increase the LDL-cholesterol, total cholesterol and triglycerides concentrations at the end of the intervention.

Conflict of interest: Authors declare no conflict of interest.

Acknowledgments: Yakult Mexico for the support with dairy food.

Keys words: symbiotic / A. salmiana / children / obesity / lipid.

\section{0 \\ LACTOBACILLUS FERMENTUM CECT5716 SUP- PLEMENTATION DURING RAT GESTATION AND LACTATION PERIODS BENEFICIALLY IMPACTS THEIR MILK AND PLASMA LIPID PROFILE}

\footnotetext{
${ }^{(1)}$ I. Azagra-Boronat; ${ }^{(2)}$ A. Tres; ${ }^{(1)}$ M. Massot-Cladera; ${ }^{(1)}$ A. Franch; ${ }^{(1)} M$. Castell; ${ }^{(2)}$ F. Guardiola; ${ }^{(1)}$ F.J. Pérez-Cano; ${ }^{(1)}$ M.J. Rodríguez-Lagunas.

(1)Sección de Fisiología. Departamento de Bioquímica y Fisiología. Facultad de Farmacia y Ciencias de la Alimentación. INSA.UB. Universidad de Barcelona. Barcelona. Spain; ${ }^{(2)}$ Departamento de Nutrición, Ciencias de la Alimentación y Gastronomía. Facultad de Farmacia y Ciencias de la Alimentación. INSA.UB. Universidad de Barcelona. Barcelona. Spain.
}

Introduction: The concentration of nutrients in human milk (e.g. vitamins, fatty acids, etc.) may be influenced by the maternal dietary intake. However, little is known about how this intake may modulate the composition of other components in breast milk, and especially those with biological action. Literature on the beneficial effects exerted by probiotics in mothers during the pregnancy and breastfeeding periods is scarce.

Objectives: To investigate whether supplementation with a probiotic isolated from human milk in rats during the gestation 
and lactation periods may influence the lipid profile of breast milk and plasma.

Methods: Lewis rats were administered daily either Lactobacillus fermentum CECT5716 or a vehicle, from the beginning of the gestation to the second week of breastfeeding ( $\mathrm{N}=6$ /group, 5 weeks). At the end of the intervention, plasma and breast milk were obtained from the dams, as well as plasma from the 14-dayold suckling rats. The lipid profiles were studied by means of a gas chromatography-flame ionization detector (GC-FID).

Results: The probiotic supplementation was able to modify the lipid profile in the plasma and breast milk of the dams and the plasma of the offspring, as observed by different clustering patterns in the principal component analysis (PCA). In brief, probiotic supplementation in dams resulted in higher levels of oleic, eicosapentaenoic and gamma-linoleic acids in plasma $(\mathrm{p}<0.05)$, whereas it led to lower palmitic acid and a rise in the proportion of linoleic and linolenic acids in breast milk $(\mathrm{p}<0.05)$. As a consequence, the suckling rats of the dams receiving the probiotic also had lower palmitic and higher linoleic acid proportions $(\mathrm{p}<0.05)$.

Conclusions: Supplementation with Lactobacillus fermentum CECT5716 during gestation and breastfeeding periods could be a good strategy to improve the lipid quality of the mother's milk composition, and therefore transmit their beneficial properties to the offspring.

Conflict of interest: Authors declare no conflict of interest.

Key words: breastmilk / probiotic / pregnancy / breastfeeding / lipids.

\section{1}

\section{GOS/FOS SUPPLEMENTATION IN SUCKLING RATS IMPROVES RV DIARRHOEA BY CHANGING THE INTESTINAL EXPRESSION OF TOLL-LIKE RE- CEPTORS AND THE MICROBIOTA COMPOSITION}

\author{
${ }^{(1)}$ M. Massot-Cladera; ${ }^{(1)}$ I. Azagra-Boronat; ${ }^{(1)}$ À. Franch; ${ }^{(1)} \mathrm{M} . J$. \\ Rodríguez-Lagunas; ${ }^{(2)}$ S. Tims; ${ }^{(2)} \mathrm{K}$. Knipping; ${ }^{(2)}$ J. Garssen; ${ }^{(1)} \mathrm{F} . J$. \\ Pérez-Cano.
}

\begin{abstract}
(1)Sección de Fisiología. Departamento de Bioquímica y Fisiología. Facultad de Farmacia y Ciencias de la Alimentación. INSA.UB. Universidad de Barcelona. Barcelona. Spain; ${ }^{(2)}$ Danone Nutricia Research. Utrecht. The Netherlands.
\end{abstract}

Introduction: The addition of a mixture of short-chain galacto-oligosaccharides (scGOS) and long-chain fructo-oligosaccharides (lcFOS) at a 9:1 ratio in infant formulas has demonstrated multiple health-promoting effects, including the modulation of the immune response, microbiota composition and infections. We have previously reported that scGOS/lcFOS ameliorates RV diarrhoea, but little is known about its mechanism of action.

Objectives: To investigate whether the amelioration of RV diarrhoea observed after the supplementation with scGOS/lcFOS was linked to changes in the intestinal innate receptors and microbiota composition.
Methods: Lewis rats were daily administered either scGOS/ lcFOS or a vehicle from days 2-8 of life. On day 5, RV was administered intragastrically. The RV group received only the infection and the REF group the vehicle. Faecal samples were collected daily for the clinical evaluation. On day 8 , the animals were sacrificed to obtain the small intestine and assess the Toll-like receptor (TLR) expression by real-time PCR. The faecal microbiota composition was studied by Illumina sequencing of V3-V4 16S rRNA.

Results: The RV induced moderate diarrhoea with an increase in TLR2 expression and affected gut microbiota composition, which was characterized by loss of bacterial groups, such as Streptococcus and Staphylococcus, and colonization by many bacteria from the Proteobacteria phylum. The supplementation with the mixture of oligosaccharides ameliorated the RV diarrhoea in terms of severity, incidence and duration and enhanced gene expression of TLR 5 and TLR9. Finally, the changes in the microbiota composition due to the challenge by RV were prevented by the supplementation with scGOS/lcFOS.

Conclusions: These results evidence that supplementation with scGOS/lcFOS ameliorates the diarrhoea induced by RV, an effect that could be mediated by the prevention of changes in the microbiota composition and the crosstalk between the microbiota and immune system.

Conflict of interest: ST, KK and JG declare that they are employees of Danone Nutricia Research.

Key words: microbiota / GOS/FOS / TLR / rotavirus / rats.

\section{2 \\ DIET-INDUCED GUT MICROBIOTA DYSBIOSIS CAN BE AMELIORATED BY FAECAL MICROBIOTA TRANSPLANT}

\author{
${ }^{(1,2)}$ M. Guirro; ${ }^{(2)} H$. Torrell; ${ }^{(2)}$ A. Costa; ${ }^{(2)} P$. Herrero; ${ }^{(2)} N$. Canela; ${ }^{(1,2)} L I$. \\ Arola. \\ (1)Nutrigenomics Research Group. Universitat Rovira i Virgili. \\ Tarragona. Spain; ${ }^{(2)}$ Centre for Omic Sciences. Joint Unit \\ Universitat Rovira i Virgili-EURECAT Technology Centre of \\ Catalonia. Unique Scientific and Technical Infrastructures. Reus. \\ Spain.
}

Introduction: Diet or antibiotics can affect gut microbiota; sometimes these alterations can provoke differences in community composition, functional genes and metabolic activities resulting in gut dysbiosis, which is an important complication in conditions such as obesity. To tackle this dysbiosis, metagenomics seems to be the most appropriate way to gain understanding of the main functions of gut microbiota and its relation to the host.

Objectives: To determine the effect of a faecal microbiota transplant (FMT) after an antibiotic treatment in rats fed with a high-fat diet (HFD), assessing changes in microbiota.

Methods: Eight-week-old male Wistar rats were divided in five groups $(n=40)$. The first group was fed with a low-fat diet (LFD) and a second group with a HFD for 9 weeks. The third and the fourth groups were fed with a LFD and HFD, respectively, for 9 
weeks and an additional 3 weeks supplemented with an antibiotic treatment (Vancomycin $25 \mathrm{mg} / \mathrm{ml}$, Neomycin and Metronidazol $50 \mathrm{mg} / \mathrm{ml}$, Ampicillin $10 \mathrm{mg} / \mathrm{ml}$ ) (LFD-ABS and HFD-ABS). Finally, the last group was fed with a HFD for 14 weeks: from 10 to 12 weeks rats received the antibiotic treatment and in the last two weeks a FMT of the LFD group (HFD-FMT). 16sRNA analysis was performed in cecal content according to next generation sequencing protocols using Ion Torrent Technology.

Results: Body weight and fat mass increased significantly in the HFD group after 9 weeks but during antibiotic treatment both parameters decreased in all groups. In the FMT period, rats started to recover weight and mass. Microbiota diversity was differentiated between diets at the family level; 3 families were the most distinctive (Clostridiaceae, Christensenellaceae, Mogibacteriaceae); moreover, it was considerably disturbed by the antibiotics treatment. Hence, the gut was recolonized by microbiota, showing that, besides diet, the microbiota was more similar to LFD than HFD, indicating FMT was successfully accomplished.

Conclusions: Diet plays a significant role in microbiota composition, but FMT can be proposed as a complementary treatment for gut dysbiosis to ameliorate obesity comorbidities.

Conflict of interest: Authors declare no conflict of interest.

Key words: microbiota / metagenomics / faecal microbiota transplant.

\section{Poster Abstract Presentations}

\section{3 \\ LACTABACILLUSRHAMNOSUS GG IMPROVES
SEVERAL IMMUNE FUNCTIONS IN OLD MICE}

${ }^{(1,2)}$ M. De la Fuente; ${ }^{(1)}$ M. De la Serna; ${ }^{(1,2)} N$. Ceprian; ${ }^{(3)}$ T. Requena.

(1)Department of Genetics, Physiology and Microbiology (Animal Physiology Unit). Faculty of Biology. Complutense University of Madrid. Madrid. Spain; ${ }^{(2)}$ Institute of Investigation of Hospital 12 de Octubre (i+12). Madrid. Spain; ${ }^{(3)}$ Department of Food Biotechnology and Microbiology. Institute of Food Science Research (CIAL-CSIC). Madrid. Spain.

Introduction: The aging process involves a loss of homeostasis, as a consequence of the deterioration of homeostatic systems, such as the nervous, endocrine and immune systems. Since the immune system is a marker of health and biological age, the control of its age-related changes (immunosenescence) is an important scientific challenge. Nutrition is a strategy to improve the homeostatic systems and aging process. In this context, supplementation with probiotics seems to be a useful tool to improve the immune system, especially in aging.

Objectives: To analyze the effect of the administration of Lactabacillus rhamnosus GG (LGG) on several functions of immune cells from old mice.

Methods: Old and adult ICR-CD1 female mice were divided into two groups ( $\mathrm{N}=8$ per group), which drank LGG $\left(10^{9} \mathrm{CFU} /\right.$ mice/day; lyophilized in skimmed milk and added to drinking water) or skimmed milk without LGG (controls). After four weeks of this treatment, peritoneal leukocytes were obtained and their capacity of phagocytosis (PI: number latex particles ingested) and chemotaxis (CI: number cells on filter) was analyzed.

Results: The results showed that both phagocytosis of macrophages as well as chemotaxis of macrophages and lymphocytes, which had lower values in old animals (PI:250 \pm 35 ; CI:300 \pm 60 ) than in adults (PI:399 \pm 73 ; CI:450 \pm 80$)(\mathrm{p}<0,001)$, indicating a typical immunosenscence, showed a significant increase after the ingestion of LGG, reaching values similar to those of adult controls. The positive effect of LGG ingestion on these immune functions was observed in adults (PI:480 $\pm 120 \mathrm{NS}$; CI:576 \pm 109 $\mathrm{p}<0.05)$ but it was stronger in old animals (PI:367 \pm 71 ; CI:454 \pm 38 ) $(\mathrm{p}<0.001)$.

Conclusions: In conclusion, LGG ingestion improved the immune functions studied and thus, it should be considered a useful probiotic for upgrading the immune system in aging and consequently for reaching a healthy longevity.

Conflict of interest: Authors declare no conflict of interest.

Acknowledgements: FIS (PI15/01787) of Institute de Salud Carlos III-Fondo Europeo de Desarrollo Regional (ISCIII-FEDER).

Key words: probiotics / immune function / aging / LGG / mice. 


\section{4}

\section{SUPPLEMENTATION WITH TWO STRAINS OF LACTOBACILLUS PARACASEI THROUGHOUT RAT EARLY LIFE MODULATES THE LYMPHOCYTE COMPOSITION IN THE INTESTINE}

\author{
${ }^{(1)}$ M. Abril-Gil; (2) M.C. de Almagro; ${ }^{(2)}$ G. Cifuentes-Orjuela; (2) J.A. \\ Moreno Muñoz; ${ }^{(2)}$ C. Rodríguez-Palmero; ${ }^{(1)}$ M. Castell; ${ }^{(1)}$ F.J. Pérez- \\ Cano; ${ }^{(1)}$ A. Franch. \\ (1)Departament de Bioquímica i Fisiologia. Facultat de Farmacia \\ i Ciències de l'Alimentació. INSA.UB. Universitat de Barcelona. \\ Barcelona. Spain; (2)Departament de Recerca Bàsica. Laboratorios \\ ORDESA. Barcelona. Spain.
}

Introduction: Probiotics have been widely described to confer a health benefit on the host when they are consumed in adequate amounts. Their effects include the maintenance of the gut barrier function and enhancement of the immune system. As early infancy is a critical stage for the immune system development, it could be an interesting time period to evaluate the probiotic actions.

Objectives: To ascertain whether the early life supplementation with two strains of Lactobacillus paracasei could modify the intestinal immune system according to IgA synthesis and composition of Peyer's patch lymphocytes (PPL).

Methods: For this purpose, newborn Wistar rats were supplemented with two specific strains of L. paracasei (ORD0681 and ORD0712) during the first 28 days of life. Non-supplemented animals were used as a reference. At the end of the study, small intestine samples were obtained to determine the antibody content as well as the PPL composition throughout multiple immunofluorescence staining and flow cytometry analysis.

Results: The results revealed that only L. paracasei ORD0681 supplementation was able to enhance intestinal IgA production ( $\mathrm{p}<0.05$ vs reference animals). In regard to PPL composition, the main changes induced by probiotic supplementation included a significant increase in NKT cell proportion by L. paracasei ORD0681 and a decrease induced by L. paracasei ORD0712 in NK and B lymphocyte percentages. Moreover, L. paracasei ORD0712 increased the proportion of cells expressing L-selectin (CD62L), a molecule involved in lymphocyte migration.

Conclusions: This study evidences that supplementation with both strains of Lactobacillus paracasei produces strain-dependent intestinal immunomodulatory effects in early life.

Conflict of interest: The authors -MCDA, GCO, JAMM and CRP- declare that they are employees of the company Laboratorios Ordesa.

Key words: probiotic / rat / immunonutrition / early life.

\section{5 \\ THE HUMAN MILK OLIGOSACCHARIDE 2'-FU- COSYLLACTOSE AMELIORATES ROTAVIRUS-IN- DUCED DIARRHOEA IN A SUCKLING RAT MODEL}

\author{
(1) I. Azagra-Boronat; (1)M. Massot-Cladera; (1)M. Castell ; ${ }^{(1)}$ M.J. \\ Rodríguez-Lagunas; ${ }^{(2)}$ B. van't Land; ${ }^{(3)} K$. Knipping; ${ }^{(3)}$ J. Garssen; ${ }^{(1)} F . J$. \\ Pérez-Cano.
}

\begin{abstract}
(1)Sección de Fisiología. Departamento de Bioquímica y Fisiología. Facultad de Farmacia y Ciencias de la Alimentación. INSA.UB. Universidad de Barcelona. Barcelona. Spain; (2)Danone Nutricia Research and University Medical Centre Utrecht/ Wilhelmina Children's Hospital. Department of Pediatric Immunology. Utrecht. The Netherlands; ${ }^{(3)}$ Danone Nutricia Research and Division of Pharmacology. Utrecht Institute for Pharmaceutical Sciences. Faculty of Science. Utrecht University. Utrecht. The Netherlands.
\end{abstract}

Introduction: Human milk oligosaccharides (HMOs) are involved in early life development by influencing immunity and intestinal health. One of the most abundant HMOs is 2'-fucosyllactose (2'-FL), representing $\sim 30 \%$ of the total oligosaccharide mixture. There is evidence suggesting a beneficial impact of 2'-FL in exerting immunomodulatory effects, inhibiting colonization of pathogens and promoting gut microbial colonization.

Objectives: To investigate the impact of 2'-FL in rotavirus (RV)-induced diarrhoea in suckling rats and to unravel mechanisms involved in such protection.

Methods: Lewis rats were daily administered 2'-FL or a vehicle from days 2-16 of life. On day 5, RV was administered intragastrically to the 2'-FL and RV groups while the REF group only received the vehicle. Faecal samples were collected daily for the clinical evaluation. On day 8, corresponding to the peak of diarrhoea, the animals were sacrificed in order to study the intestinal architecture. To assess the ability of 2'-FL to bind the RV, different dilutions of 2'-FL were preincubated with the virus $(5 \times 104$ viral particles $/ \mathrm{mL}$ ) at $1 / 1$ ratio for $30 \mathrm{~min}$. Subsequently, free, noncoated viral particles were quantified by ELISA.

Results: The supplementation with 2'-FL ameliorated the $\mathrm{RV}$-induced diarrhoea in terms of severity, incidence and duration. Although the RV infection alone did not alter the intestinal architecture, the supplementation with 2'-FL resulted in higher villi height and area compared to REF animals. Moreover, the 2'FL was able to bind to the RV, as seen by a moderate inhibition $(\sim 10 \%)$ in the ELISA-blocking-assay.

Conclusions: Supplementation with 2'-FL in early life ameliorates $\mathrm{RV}$-induced diarrhoea. Such an effect may be linked to an effect of 2'-FL on the gut maturation and a RV blocking effect of the oligosaccharide, thus inhibiting the progression.

Conflict of interest: BvL, KK and JG declare that they are employees of Danone Nutricia Research.

Key words: rotavirus / prebiotic / histology / rat / HMO. 
096

\title{
NEW FORMULA SUPPLEMENTED WITH MILK FAT GLOBULE MEMBRANE AND SYNBIOTICS MODULATES THE GUT MICROBIOTA AND RE- DUCES ILLNESS SYMPTOMS DURING INFANCY
}

\author{
${ }^{(1)}$ C. Campoy; ${ }^{(2)}$ T. Cerdó; (1) A. Nieto-Ruiz; ${ }^{(1)}$ F. Hermann; ${ }^{(3)}$ J. J \\ imenez; ${ }^{(1)} \mathrm{A}$. Suárez. \\ (1)Department of Pediatrics. University of Granada. Granada. \\ Spain; ${ }^{(2)}$ Biochemistry and Molecular Biology. University of \\ Granada. Granada. Spain; ${ }^{(3)}$ Ordesa Laboratories. Barcelona. \\ Spain.
}

Introduction: Infancy is a critical period for organ maturation and long-term growth. Observational studies have provided evidence about how alterations in early nutrition, from breast feeding or formulas, can produce disturbances in the pathways that regulate energy expenditure, the immune system and other systemic effects, promoting the development of certain diseases and altering the establishment and maturation of gut microbiota.

Objectives: To compare the effect of a standard infant formula (F1) with a new one designed by Ordesa Laboratories SL and supplemented with LC-PUFAs, Milk Fat Globule Membrane (MFGM) components and synbiotics (Nutriexpert ${ }^{\oplus}$ factor) (F2) on the gut microbiota composition of infants during the first 18 months.

Methods: 170 fecal samples were obtained from healthy term infants enrolled in a randomized double-blind study to receive a standard infant formula ( $F 1: n=85)$ or the new one $(F 2: n=85)$. Breastfed infants were included as a control group (BF: $n=50$ ). Gut microbiota composition was assessed by $16 \mathrm{~S}$ rRNA gene sequencing at 1, 6, 12 and 18 months of age and disease registry was recorded by questionnaires. Statistical analyses were performed using $\mathrm{R}$ and SPSS.

Results: We observed that infants receiving F2 showed fewer respiratory tract infections $(\mathrm{p}=0.033)$ and total infections $(\mathrm{p}=0.018)$ compared to $\mathrm{F} 1$ and BF infants. At 12 months of life, $\mathrm{BF}$ infants showed a higher rate of conjunctivitis $(\mathrm{p}=0.042)$ and unclassifiable febrile episodes $(p=0.005)$ than infants fed F2. At 18 months of life, infants fed F2 showed a lower rate of conjunctivitis $(\mathrm{p}=0.013)$ than BF infants. Regarding gut microbiota composition and structure (assessed by Unifrac distance metrics), we observed that the gut microbiota of F2-fed infants was more similar to that of breast-fed than F1-fed ones. F2-fed infants had significantly higher levels of Lactobacillus sp. with respect to F1.

Conclusions: The new formula mimicked the beneficial effects of breast milk more closely, reducing illness episodes in infants and promoting the growth of probiotic microorganisms. Conflict of interest: Authors declare no conflict of interest.

Key words: symbiotics / infancy / formula / microbiota / infections. 


\section{Nutritions of Metabolism}

\author{
Reviewers: \\ Margarita Castell Escuer \\ Universitat de Barcelona \\ Rosaura Farré Rovira \\ Universidad de Valencia \\ Benjamín Martín Martínez \\ Sociedad Española de Investigación en Nutrición y Alimentación \\ en Pediatría
}

Montserrat Rivero Urgell

Associació Catalana de Ciències de l'Alimentació

\section{Oral Abstract Presentations}

\section{7 \\ WHAT ABOUT LEGUMES AS A PLANT SOURCE OF THE DAO ENZYME?}

O. Comas-Basté; S. Sánchez-Pérez; R.I. Garza-Guajardo; M.L. Latorre-Moratalla; M.T. Veciana-Nogués; M.C. Vidal-Carou.

Departament de Nutrició, Ciències de l'Alimentació i Gastronomia. Facultat de Farmàcia i Ciències de l'Alimentació. INSA.UB. XaRTA. Universitat de Barcelona. Santa Coloma de Gramenet. Spain.

Introduction: The use of exogenous diamine oxidase (DAO) enzyme has been recently postulated as a potential strategy for the treatment of histamine intolerance, a disorder in the homeostasis of histamine caused by a reduction of its intestinal degradation. Dietary supplements based on gastro-resistant encapsulated porcine kidney protein extract are available on the market to reduce the symptoms of this intolerance. Although with scarce scientific research, there are some references about the potential of pea seedlings as a source of DAO.

Objectives: To study the capacity of legumes and their sprouts to reduce histamine in vitro and evaluate the influence of different growing conditions on this enzymatic activity. If confirmed, legumes could become an advantageous alternative to porcine DAO enzyme from a productive and sustainable perspective.

Methods: In vitro DAO activity was measured through an enzymatic assay and the subsequent analysis of remaining histamine by UHPLC-FL. Analysed samples were both raw pulses and sprouts of lentils, beans (white, red and black), broad beans, peas, chickpeas and soy.

KARGER XVII Conference of SEÑ and X Meeting of ACCA
Results: Histamine-degrading capacity was found both in raw pulses and sprouts of some legumes. Lentils, broad beans and white, red and black beans showed in vitro DAO activity, ranging from 0.32 to $1.95 \mathrm{mU} / \mathrm{g}$ (nmols of degraded histamine per minute/g of legume). This activity was absent in raw peas, chickpeas and soy. Etiolated sprouts (grown in darkness) of various legumes showed higher DAO activity in comparison with raw pulses, with mean values of $40.25 \pm 8.1 \mathrm{mU} / \mathrm{g}$ in chickpea sprouts, $35.2 \pm 5.3$ $\mathrm{mU} / \mathrm{g}$ in lentil sprouts and $27.5 \pm 1.3 \mathrm{mU} / \mathrm{g}$ in pea sprouts.

Conclusions: Results confirm the ability of various raw pulses and legume sprouts to reduce histamine in vitro and their potential as a plant source of the DAO enzyme. Further studies are needed to better establish the influence of growing conditions of the sprouts and extraction treatments on DAO activity.

Conflict of interest: Authors declare no conflict of interest.

Key words: histamine / histamine intolerance / DAO / legumes / sprouts.

\section{8 \\ LOW-HISTAMINE DIET SUPPLEMENTED WITH EXOGENOUS DIAMINE OXIDASE ENZYME IS USEFUL FOR TREATING MIGRAINE IN PATIENTS WITH DAO DEFICIENCY}

${ }^{(1)}$ A. Duelo; ${ }^{(1)}$ M. Berbel; ${ }^{(1)} H$. Mantecon-Laviguerie; ${ }^{(2)}$ O. Comas-Basté; ${ }^{(2)}$ M.L. Latorre-Moratalla; (2)M.T. Veciana-Nogués; ${ }^{(2)}$ M.C. Vidal-Carou.

(1)Department of Nutrition. Instituto Clínico del Déficit de DAO (ICDDAO). Sant Cugat del Vallès. Spain; ${ }^{(2)}$ Departament de Nutrició, Ciències de l'Alimentació i Gastronomia. Facultat de Farmàcia i Ciències de l'Alimentació. INSA.UB. XaRTA. Universitat de Barcelona. Santa Coloma de Gramenet. Spain.

Introduction: Low-histamine diets and/or exogenous diamine oxidase $(\mathrm{DAO})$ supplementation are currently used to treat symptoms of histamine intolerance $(\mathrm{IH})$, a disorder in histamine homeostasis that increases its plasma levels, mainly due to DAO deficiency. Headache is the most recognized symptom.

Objectives: To assess the effectiveness of a low-histamine diet plus a DAO enzyme supplement on the remission of migraine in subjects with DAO deficiency.

Methods: An intervention study was carried out in 212 individuals with a migraine diagnosis by a neurologist according to the International Classification of Headache Disorders and with DAO deficiency (DAO $<80 \mathrm{HDU} / \mathrm{ml}$ ). Subjects followed a 3-month low-histamine diet based on the exclusion of foods considered rich in histamine or other biogenic amines and usually related to the onset of HI symptoms, such as fermented products (cheese, 
dry-fermented sausage, wine, beer), semi-preserved and canned fish, shellfish, certain fruits and vegetables (spinach, tomato, eggplant, avocado, citrus, bananas, strawberries, pineapple, nuts) and chocolate $^{1,2}$. A DAO supplement was administrered before breakfast, lunch and dinner. Outcomes assessed were duration and number of attacks and perception of pain intensity with a scorescale from 0 (absence) to 10 .

Results: Most subjects showed an improvement in migraines after the 3-month treatment: $34.9 \%$ reported complete remission and another $35.8 \%$ had a reduced number of migraine episodes per month, of less duration and pain intensity. The treatment was less successful in $29.3 \%$ of patients. On average, when comparing baseline and final values after treatment, all outcomes were significantly reduced: 8 to 2 attacks per month, 24 to 3 hours of pain and 8 to 4 in pain intensity scoring.

Conclusions: A low-histamine diet supplemented with the DAO enzyme for three months was useful in reducing the number of attacks, duration and intensity of pain in migraineous patients with DAO deficiency.

References: ${ }^{1}$ Rosell-Camps et al. (2013); ${ }^{2}$ Wagner et al. (2017).

Conflict of interest: Authors declare no conflict of interest.

Key words: DAO / histamine / low-histamine diet / migraine.

\section{Poster Abstract Presentations}

\section{9 \\ FOOD ALERT NETWORK AND ITS RELATION- SHIP WITH ADVERSE REACTIONS TO FOOD}

V. Torres; J. García; J.A. Sánchez-Rodríguez; L. Macías.

Unidad de Protección de la Salud. Área Sanitaria Norte de

Málaga. Hospital de Antequera. Antequera. Spain.

Introduction: In Andalucía, the possibility of suffering an anaphylactic shock due to food intake has quadrupled in the last two decades. Adverse reactions due to allergies and food intolerances are thus a public health problem in our Autonomous Community.

We have distinguished 14 substances with a higher risk of causing food allergy, so their inclusion in nutrition labeling is mandatory. Since 2008, specific allergen control programs have been carried out on foods marketed in Andalucía.

Objectives: To analyze the situation of food alerts due to the presence of undeclared allergens as a basis for action in those sectors with the greatest involvement. To contribute to a high degree of health protection against food-borne risks, and to promote the quality of the environment where people live

Methods: Source of information: Database of alerts network of the Área Sanitaria Norte de Málaga (ASNM). Categorization of foods according to the Guide for the Operation of the General Sanitary Registry of Food and Food Companies.

Procedure of action according to the ALERT PROCESSING Manual (Ministry of Health of the Junta de Andalucía).

Results: The number of alerts in our ASNM related to allergens has risen significantly in recent years. The foods with the greatest implication are of plant origin (cereals).

Conclusions: The increase of alerts coincides with the implementation of Regulation 1169/2011 and is related to the agri-food characteristics of our Autonomous Community.

Conflict of interest: Authors declare no conflict of interest.

Key words: food allergy / public health / alerts. 


\section{Nutritions of Metabolism}

\author{
Reviewers: \\ Estanislau Fons Solé \\ Universitat de Lleida \\ Joan Tibau Font \\ Institut de Recerca i Tecnologia Agroalimentàries \\ Miquel Moretó Pedragosa \\ Universitat de Barcelona \\ José Juan Rodríguez Jerez \\ Universitat Autònoma de Barcelona
}

\section{Oral Abstract Presentations}

\section{0 \\ SHOTGUN TRIACYLGLYCEROL PROFILING AND CHEMOMETRICS: TOWARDS A FAST AUTHENTI- CATION OF OLIVE OIL ADULTERATION}

\author{
${ }^{(1)}$ B. Quintanilla-Casas; ${ }^{(1)}$ S. Vichi; (1) J. Bustamante; ${ }^{(2)}$ M. Simon; ${ }^{(3)}$ F. \\ Guardiola; ${ }^{(3)}$ A. Tres. \\ (1)Department of Nutrition, Food Science and Gastronomy. \\ Food and Nutrition Torribera Campus. Universitat de Barcelona. \\ Santa Coloma de Gramenet. Spain; ${ }^{(2)}$ Federació de Cooperatives \\ Agràries de Catalunya (FCAC). Barcelona. Spain; ${ }^{(3)}$ Department of \\ Nutrition, Food Science and Gastronomy. Faculty of Pharmacy \\ and Food Sciences. Universitat de Barcelona. Barcelona. Spain.
}

Introduction: Olive oil (OO) tops the list of current adulterated food products due to its high value. One of the most common fraudulent commercial practices is the blending with oils of different botanical origin, which can be a food safety issue because of loss of product traceability. For this reason, disposing of fast and high throughput methods for an efficient wide-ranging screening analysis would support the detection of illegal OO blends.

Objectives: To develop authentication models using triacylglycerol (TAG) profiles obtained by shotgun high-resolution mass spectrometry., This approach allows the exact masses of many components to be obtained in only one analysis and in a short time, including minor TAGs not detected by conventional techniques.

Methods: The experiment followed the Balanced Incomplete Latin Square design, in which 60 genuine oils were mixed with 25 high-linoleic oils (soy and sunflower) at $2 \%$ and $5 \%$ and with 20 high-oleic oils (hazelnut and high oleic sunflower) at 5\% and $10 \%$

\footnotetext{
KARGER XVII Conference of SEÑ and X Meeting of ACCA
}

$(\mathrm{n}=240)$. Two Partial Least Square-Discriminant Analysis (PLSDA) authentication models were performed according to the type of adulterant described above.

Results: PLS-DA prediction results in an internal validation by leave-10\%-out cross validation were satisfactory: all genuine oils were unequivocally distinguished from OO blends containing $\geq 2$ $\%$ of high-linoleic and $\geq 5 \%$ of high-oleic adulterants.

Conclusions: This prospective study indicates that the proposed method is a promising tool to detect illegal blending of $\mathrm{OO}$ with low percentages of other vegetable oils.

Conflict of interest: The authors declare no conflict of interest.

Acknowledgements: This project has been funded by ACCIÓ-Generalitat de Catalunya and the European Union (Programa Operatiu FEDER Catalunya 2014-2020) in the framework of the project Autenfood (Ref COMRDI-15-1-0035). It has also been supported by the Spanish MINECO (JCI-2012_13412) and MECD (FPU16/01744).

Key words: olive oil / triacylglycerols / authentication / UHRMS.

\section{1}

\section{PRESENCE OF ANISAKIDS (NEMATODA) IN COMMONLY CONSUMED FISH FROM THE SPAN- ISH ATLANTIC AND MEDITERRANEAN COASTS AND THEIR HEALTH RISK IMPLICATION}

\author{
${ }^{(1)} X$. Roca-Geronès; ${ }^{(2)}$ M. Segovia; ${ }^{(1)} \mathrm{C}$. Godínez-González; ${ }^{(1)}$ R. Fisa; ${ }^{(1)}$ I. \\ Montoliu. \\ (1)Laboratory of Parasitology. Department of Biology, Health and \\ Environment. Faculty of Pharmacy and Food Sciences. University \\ of Barcelona. Barcelona. Spain; (2)Department of Health. Miquel \\ Martí i Pol Institute. Generalitat de Catalunya. Barcelona. Spain.
}

Introduction: The presence of anisakid larvae in fish may cause the human infection named anisakidosis, characterized by gastrointestinal pathology and/or allergy. Its incidence has increased in Europe due to the growing habit of consuming marinated/raw fish.

Objectives: The aim of the study was to analyse the presence of anisakids in fish habitually consumed in Spain, with special emphasis on spatial distribution in the edible parts and its implication on human health.

Methods: 93 blue whiting (Micromessistius poutassou) and 52 horse mackerel (Trachurus trachurus) from Atlantic and Mediterranean Spanish coasts and purchased in Barcelona markets were analysed. Viscera were observed in fresh fish and flesh was digest- 
ed in pepsin- $\mathrm{HCl}$ solution. Microscopical identification of larvae was based on the morphology of the main structures of the digestive tract and the anatomy of anterior and posterior ends.

Results: Two anisakid species, Anisakis simplex (s.l.), the main causative agent of anisakidosis, and Hysterothylacium aduncum, were detected. Anisakid prevalence (P) and mean intensity (MI) were influenced by the fish origin, being significantly higher $(\mathrm{p}<0.05)$ in the Atlantic (P: 84-100\%; MI: 30.5-44) than in the Mediterranean (P: 34-66\%; MI: 1.6-3.8), and by host length and weight in T. trachurus specimens $(\mathrm{p}<0.05)$. Larvae were located mainly in the viscera, Atlantic fishes showing larvae in muscle, especially the blue whiting (A. simplex, P: 59\% MI: 10.3; H. aduncum P: 50\% MI: 4.2). A. simplex parasitation affected principally the antero-ventral part of the musculature, the infection decreasing when approaching the tail. The muscular distribution pattern of $H$. aduncum was similar but with lower intensities $(p<0.05)$.

Conclusions: The significant presence of A. simplex in studied fishes constitutes a risk for consumers, especially noticeable in Atlantic fishes with a considerable load in flesh. H. aduncum, although considered non-pathogenic, usually co-infects consumed fish with Anisakis; its similar morphology can lead to erroneous diagnoses, which should be taken into account.

Conflict of interest: Authors declare no conflict of interest.

Acknowledgements: 2018-SGR-1008-GeneralitatCatalunya.

Key words: anisakidae / fish / Atlantic / Mediterranean / Spain.

\section{2}

\section{ANISAKIS LARVAE QUANTIFICATION IN COM- MERCIAL FISH AND FISH-DERIVED FOOD USING A MOLECULAR APPROACH}

\author{
C. Godínez-González; X. Roca-Geronès; I. Montoliu; R. Fisa.
}

Laboratory of Parasitology. Department of Biology, Health and Environment. Faculty of Pharmacy and Food Sciences. University of Barcelona. Barcelona. Spain

Introduction: The presence of Anisakis in fish has become a food safety problem. Anisakiosis is caused by larva ingestion in raw or undercooked fish. Furthermore, Anisakis species are recognized as causative agents of allergic reactions, elicited by proteins from live and dead larvae. To develop reliable detection methods to ensure consumer health is of paramount importance.

Objectives: To optimize a mathematical model to estimate the number of larvae of Anisakis species in fish and fish-derived food by a specific qPCR assay.

Methods: Anisakis-free hake fillets ( $15 \mathrm{~g}$ ) were experimentally contaminated with different numbers of A. simplex larvae (1-50). DNA was extracted from $200 \mu \mathrm{l}$ of food homogenate in duplicate. Molecular analysis was performed using a SYBRGreen qPCR assay (cytochrome c oxidase II gene). Results were expressed in Cq values. The method was applied to analyze the edible part of 42 blue whiting fish in parallel with visual inspection and microscopic identification. Commercial fish-derived food, including 5 brands of gulas, 6 of crab sticks and 3 of croquettes, were also analyzed.
Results: Molecular results of experimentally spiked samples showed a logarithmic relation between $\mathrm{Cq}$ values and the number of larvae. A mathematical model was obtained, allowing the detection of larval traces and the quantification of 1-35 larvae $(\mathrm{R} 2=0.9504, \mathrm{CV}=3.62 \%)$. Regarding blue whiting fish, the number of larvae estimated by the mathematical model corresponded to that detected by visual inspection $(\mathrm{r}=0.92)$. Bland-Altman analysis showed a $95 \%$ agreement between both methods. In fish-derived food, less than one larva was detected in $80 \%$ of crab sticks, $66 \%$ of gulas and $33 \%$ of croquettes.

Conclusions: The optimized technique could be applied for Anisakis detection and quantification in fish and fish-derived food, regardless of the industrial processing, improving automation of routine food safety and quality control.

Conflict of interest: Authors declare no conflict of interest.

Acknowledgements: 2018-SGR-1008-GeneralitatCatalunya and CONACYT-Mexico.

Key words: Anisakis / QPCR / seafood / food safety. 
Poster Abstract Presentations

\section{3 \\ GROWTH INHIBITION OF LISTERIA MONOCY- TOGENES BY A CELL-FREE EXTRACT OF CUL- TURED BACTERIA ISOLATED FROM A MEAT PROCESSING PLANT}

\section{A.S. Hascoët; C. Ripollés; A. Guerrero; M. Martínez; J.J. Rodríguez.}

Departamento de Ciencia Animal y de los Alimentos. Facultad de Veterinaria. Universidad Autónoma de Barcelona. Barcelona. Spain.

Introduction: Listeria monocytogenes is still a contemporary problem. In December 2017, an enormous outbreak of listeriosis started in South Africa, with nearly 750 laboratory-confirmed cases and 67 deaths. The ability of this pathogen to form biofilms makes its elimination a challenge for food companies. In natural environments, such as in the food industry, biofilms are formed by multiple bacterial species and they have been found to be more resistant to disinfectants and sanitizers than mono-species biofilms.

Objectives: To find Listeria monocytogenes inhibitory substances produced by bacteria naturally present in a meat processing plant.

Methods: This study started by identifying potential inhibitory bacteria from the microbiota of a pork meat cutting plant. The growth of four wild strains of L. monocytogenes was challenged with a cell-free extract of two possible inhibitors. The test was performed in an Eppendorf joining $150 \mu \mathrm{l}$ of TSB (inoculated at 0.1 Mc Farland of each strain of L. monocytogenes) and $150 \mu \mathrm{l}$ of a cell-free extract from each probable inhibitor culture medium in different concentrations $(10,20,30$ and $40 \%)$. The absorbance of $300 \mu \mathrm{l}$ of each test was measured with a Bioscreen C every $30 \mathrm{~min}$ for $48 \mathrm{~h}$, incubated at $30^{\circ} \mathrm{C}$ with continuous shaking to evaluate the L. monocytogenes growth.

Results: One of the four strains of L. monocytogenes was significantly affected by the cell-free extract from a potential inhibitory microorganism. As the concentration of the extract was increased, the exponential phase of growth was delayed. This strain of L. monocytogenes, when exposed to the highest concentration of cell-free extract tested, needed $18 \mathrm{~h}$ more to start the exponential phase in comparison with the control.

Conclusions: One of the potential inhibitor microorganisms was able to produce substances that delayed L. monocytogenes development, but it was not sufficiently effective to control this pathogen in a lasting way. Further studies are needed to characterize these inhibitory molecules.

Conflict of interest: Authors declare no conflict of interest.

Keys words: Listeria monocytogenes / inhibition / cell-free extract.

\section{4 \\ TRAINING IN FOOD SAFETY WITHIN THE CON- TEXT OF EDUCATION FOR CITIZENSHIP}

\author{
V. Torres; L. Macías; J. García; J.A. Sánchez-Rodríguez. \\ Unidad de Protección de la Salud. Área Sanitaria Norte de \\ Málaga. Hospital de Antequera. Antequera. Spain.
}

Introduction: "Education for citizenship" guides the educational function towards the development of people with a critical vision of their environment, who act as active members of their health. Food Safety Education includes the hygienic-sanitary quality of the food, prior to any other health consideration. In this attempt to shape healthy beliefs and behaviours, there are conditioning factors such as the integration of people from diverse cultural backgrounds and / or precariousness in health, which is still underlying in more disadvantaged sectors.

Objectives: To provide knowledge to support scientifically sustainable food health habits and attitudes. To teach selected information, providing people with the means to improve their health and change risky behaviours.

Methods: In the Área Sanitaria Norte de Málaga (ASNM), we applied our resources (professionals, physical space, time, etc.), knowledge and experience to enable Public Health information to be transmitted with the highest professional rigor. An explanatory and bidirectional methodology was applied. The activities were developed inside and outside health centers. The issues that concern the population, establishing priority objectives and the target population were defined. Channels were selected, and means made available. Messages tailored to the target population were designed

Results: Among the Programming of Health Promotion activities projected by the ASNM, it was only possible to obtain results from those that allow the extraction of necessary quantitative data to evaluate the impact of the training provided. Educational activities in the classrooms: information on the number of schools where lectures on healthy eating and hygienic habits are given and the evaluation of the results of the surveys before and after the activity given. Evaluation of menus in schools: adaptation of school canteens to the food regulations and comparison of results of 1 st and 2 nd nutritional advice and sanitary control visits. Public Health Blog: number of queries that citizens make through this internet tool. Advice and control of food safety in food manufacturing industries: number of industries inspected and / or audited and evolution of percentages of inspections and/or conforming audits over a period of time. Training and environmental sensitivity of health personnel in achieving the objectives of the environmental policy of the Andalusian Health Service: degree of compliance with the annual environmental training plan in health centers. Other non-quantifiable health promotion instruments are: dissemination of articles in the press and local radio, citizen participation plan, posters in health centers alluding to the environmental policy of SAS, etc.

Conclusions: Greater impact on health is achieved by emphasizing the design of new communication strategies, more participative communication models and the involvement of people as active subjects.

Ann Nutr Metab 2018;73 (suppl 2): 1-93 
Conflict of interest: Authors declare no conflict of interest. Key words: citizenship / food safety / education.

\section{5 \\ LISTERIA MONOCYTOGENES AND SALMO- NELLA TYPHIMURIUM BIOFILM DETACHMENT FROM STAINLESS STEEL SURFACES THROUGH ENZYMATIC TECHNOLOGY}

\author{
C. Ripolles-Avila; A.E. Guerrero-Navarro; A.S. Hascoët; J.J. Rodríguez- \\ Jerez.
}

Departamento de Ciencia Animal y de los Alimentos. Universitat Autònoma de Barcelona. Barcelona. Spain.

Introduction: Bacterial capacity to adhere to surfaces and subsequently trigger biofilm formation has significant implications within the food industry, where it is a persistent source of contamination.

Objectives: This study aims to evaluate different enzymatic formulas for use as cleaning products to treat food industry surfaces for the removal of biofilms of two pathogenic bacteria $\mathrm{L}$. monocytogenes and S. Typhimurium.

Methods: Biofilms were produced by culturing L. monocytogenes CECT 935 and S. Typhimurium CECT 722 in TSYEBgluc1 $\%+\mathrm{NaCl} 2 \%$ and $\mathrm{BPW}$, respectively. From these suspensions, $30 \mu \mathrm{l}$ were inoculated on stainless steel coupons. Surfaces were incubated at $30^{\circ} \mathrm{C}$ for a week and with renewal of culture medium at $48 \mathrm{~h}+24 \mathrm{~h}+24 \mathrm{~h}+72 \mathrm{~h}$. Different enzymatic formulas were tested, which consisted of solutions made up of protease, lipase, amylase and some including natural extracts. The treatments were applied at $50^{\circ} \mathrm{C}$ for $30 \mathrm{~min}$ or $50^{\circ} \mathrm{C}$ for $30 \mathrm{~min}+30^{\circ} \mathrm{C}$ for $15 \mathrm{~min}$, depending on the product. Direct epifluorescent microscopy was employed for the evaluation of the biofilm detachment and elimination.

Results: Biofilms were formed in the mature stage with bacterial counts ranging from 6.7 to $6.99 \log \mathrm{CFU} \mathrm{cm}-2$ for L. monocytogenes and S. Typhimurium. The percentage of detachment varied from $40 \%$ to $80 \%$, its effectivity depending on the bacteria and enzymatic product. The most effective formula contained amylase, which was found to disaggregate the biofilm structure. The effect of all formulas was enhanced when performing three washings in a row, obtaining instead of $80 \%$ a $98.43 \%$ of biofilm detachment for L. monocytogenes.

Conclusions: Based on the results obtained and the literature survey, this effectiveness can be exploited as a potent technology to control and eradicate biofilms in the food industry.

Conflict of interest: Authors declare no conflict of interest.

Key words: biofilms / pathogens / enzymatic technology / sanitisation.

\section{6 \\ MEASURING THE FOOD ENVIRONMENT OF A MEXICAN UNIVERSITY}

\author{
${ }^{(1)}$ G. Palos; ${ }^{(1)}$ A. Ayala Esqueda; (1)O. González Acevedo; ${ }^{(1)}$ E. Ibarra \\ Zapata; ${ }^{(2)}$ L. Hernández Barrera. \\ (1)Universidad Autónoma de San Luis Potosí. Facultad de \\ Enfermería y Nutrición. San Luis Potosí. México; ${ }^{(2)}$ Instituto \\ Nacional de Salud Pública de México. Morelos. México.
}

Introduction: The food environment plays an important role in influencing food choices. Access to healthy, safe and affordable food reduces chronic diseases and contributes to good health in college students.

Objectives: To measure a university food environment.

Methods: This was a cross-sectional and spacial georeference study. We carried out a mapping to identify healthy food retail and service venues within 600 meters of a buffer zone of the Universidad Autónoma de San Luis Potosí. Data were collected via a Nutritional Environment Measure Survey of restaurants.

Results: The university food environment had $91 \%$ of unhealthy food retail and service venues.

Conclusions: The university food environment was not conducive to healthy food choices. This was reflected in the high sales of relatively unhealthy foods in and around the university. Food programmes and policies on availability and prices are urgently needed.

Conflict of interest: Authors declare no conflict of interest.

Key words: nutritional / food / environment / college / access.

\section{7 \\ BREACHES IN THE LABELLING OF FOOD ES- TABLISHMENTS AUDITED IN THE HEALTH AREA NORTH OF MALAGA}

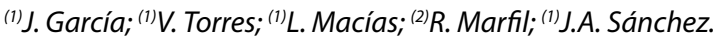 \\ (1) Unidad de Protección de la Salud. Área Sanitaria norte \\ de Málaga. Antequera. Spain; ${ }^{(2)}$ Distrito Sanitario Granada \\ Metropolitano. Granada. Spain.
}

Introduction: The labelling of foodstuffs is an important instrument for the protection of consumers. It provides information on the nature and characteristics of such products, allowing consumers to buy and eat them safely.

Objectives: To assess the degree of compliance with the Regulation (EC) 1169 / 2011 for labelling of foodstuffs in food establishments audited during 2017 in the health area north of Malaga.

Methods: A cross-sectional descriptive study was carried out during 2017. The study population consisted of 55 randomly selected food establishments with HACCP. The variables studied were the mandatory terms laid down in Regulation (EC) 1169 / 2011 except the nutritional value. The collection and analysis of 
data was carried out with the program R.2.10. Frequencies and percentages were used in the description of qualitative variables. To determine the statistically significant differences between types of establishments the Chi-square test was performed. The results were considered significant when they presented a $\mathrm{p}<0.05$.

Results: $83.3 \%$ of supervised institutions showed deficiencies in food labelling: butchers and delicatessens (40.0\%), restaurants $(20.0 \%)$ and meat industries (17.8\%). There were significant differences between the different types of establishments $(\mathrm{p}<0.05)$. By types of endorsements, there were deficiencies in the declaration of allergens $(74.1 \%)$, food (53.7\%), name, list of ingredients (48.1\%), conditions of conservation or utilization $(35.2 \%)$, date of minimum durability (29.6\%), amount of certain ingredients (25.9\%) and identification of the company (24.1\%).

Conclusions: Food business operators should review the labelling of foods they supply.

Conflict of interest: Authors declare no conflict of interest.

Key words: labelling / food stuffs / supervision.

\section{8}

\section{DECLARATION OF ALLERGENS: MAIN BREACH IN THE LABELLING OF FOOD ESTABLISHMENTS AUDITED IN THE HEALTH AREA NORTH OF MAL- AGA}

\author{
(1)J. García; ${ }^{(1)}$ V. Torres; ${ }^{(1)}$ L. Macías; ${ }^{(2)}$ R. Marfil; (1)J.A. Sánchez. \\ (1) Unidad de Protección de la Salud. Área Sanitaria Norte \\ de Málaga. Antequera. Spain; (2)Distrito Sanitario Granada \\ Metropolitano. Granada. Spain.
}

Introduction: The declaration of allergens on the labelling of foodstuffs is an important instrument for the protection of consumers, providing the necessary information for consumption.

Objectives: To ascertain the degree of compliance with the declaration of allergens in food establishments in the North of Malaga health area.

Methods: In a descriptive cross-sectional study, the study population were food establishments with the HACCP system. 55 establishments were randomly selected. The variable studied was the declaration of allergens. The collection and analysis of data was carried out with the program R.2.10. The description of qualitative variables used frequencies and percentages. To determine the existence of statistically significant differences between types of establishments the Chi-square test was performed. The results were considered significant when they presented a $\mathrm{p}<0.05$.

Results: Establishments that most breached the declaration requirement were butchers and delicatessens $(40.0 \%)$, and restoration $(20.0 \%)$ and meat $(17.8 \%)$ industries, with significant differences found between the different types of establishments. These shortcomings were not due to a deliberate action of the food establishments, and were corrected immediately after detection.

Conclusions: The results indicate that a large number of companies do not comply with the regulations. Training and revision of labelling are required. The compulsory declaration of allergens is the regulation most breached, which can pose a risk to the health of individuals with allergies or intolerance to certain foods.

Conflict of interest: Authors declare no conflict of interest. Key words: allergens / labelling / food establishments. 


\section{Reviewers:}

Estanislau Fons Solé

Universitat de Lleida

Marius Rubiralta Alcañiz

Universitat de Barcelona

\section{Oral Abstract Presentations}

\section{9 \\ A FOOD GUIDE TO FOLLOWING A HEALTHY AND ECO-FRIENDLY DIET}

\section{U. Fresán; J. Sabaté.}

School of Public Health. Loma Linda University. Loma Linda. USA.

Introduction: According to a FAO definition, diets should be both healthy and environmentally sustainable. In general, people are mindful about the health effects of their diet, but do not seem aware of the environmental effects of the food they consume. Knowledge of both potential effects may change their dietary patterns.

Objectives: To classify food groups in relation to healthiness and eco-friendliness in a simple way, which could be used by the general population to assess their food consumption.

Methods: We established food groups that most people can easily identify and classify all foods they consume among them. We reviewed the recent literature to assess how the consumption of the identified food groups affects health and how their production affects greenhouse gas (GHG) emissions. We then classified food groups as healthy, neutral or unhealthy, and low, medium or high GHG emitters.

Results: Of the 28 food groups, 6 were healthy and low GHG emitters (fruits, vegetables, beans, whole grains, dairy substitutes and water), and 3 were unhealthy and high GHG emitters (processed meat, red meat and animal fats). 1 was healthy but a high GHG emitter (fish), and 1 unhealthy but a low GHG emitter (candies).

Conclusions: The proposed Health\&Environmental guide may be a simple tool that could be used to provide information to motivated individuals regarding the health and environmental impacts of their food habits. Its effectiveness in guiding and changing food consumption/choices remains to be tested.

\footnotetext{
KARGER XVII Conference of SEÑ and X Meeting of ACCA
}

Conflict of interest: Authors declare no conflict of interest. Key words: food guide / healthy diet / sustainability.

\section{0 \\ CAVA LEES AS PROMOTERS OF THE GROWTH OF FERMENTATIVE AND PROBIOTIC BACTERIA}

S. Hernández-Macias; M.L. Latorre-Moratalla; O. Comas-Basté; S. Sánchez-Pérez; M. Riu-Aumatell; M.T. Veciana-Nogués; E. LópezTamames; M.C. Vidal-Carou.

Departament de Nutrició, Ciències de l'Alimentació i Gastronomia. Facultat de Farmàcia i Ciències de l'Alimentació. Universitat de Barcelona. Santa Coloma de Gramenet. Spain.

Introduction: Cava (natural sparkling wine) lees are non-viable cells of Saccharomyces cerevisiae, naturally plasmolyzed during cava processing ${ }^{1}$. They are currently generated at 300 tons/year as a sub-product without added value. Lees are rich in soluble and insoluble fibers and may favor the growth of probiotic and fermentative microorganisms ${ }^{2}$. A faster growth could have a positive impact on the safety of fermented food products by accelerating the $\mathrm{pH}$ drop.

Objectives: To evaluate the in vitro effect of cava lees as growth promoters of lactic acid bacteria (LAB) used as probiotic or starter cultures.

Methods: The effect of lees $(10 \% \mathrm{w} / \mathrm{v})$ on two LAB species in comparison with controls without lees was studied. The bacteria evaluated were 8 different strains of Lactobacillus sakei and L. plantarum. Microbial counts and $\mathrm{pH}$ were determined at different growth times ( 0 to 78 hours) after incubation in MRS at $30^{\circ} \mathrm{C}$ and $200 \mathrm{rpm}$.

Results: The effect of lees was different depending on the strain. Lees increased $(p<0.05)$ the growth of L. sakei, with differences of more than $1.5 \log \mathrm{CFU} / \mathrm{mL}$ (up to $28 \%$ ) with respect to the control. Higher acidification in the medium with lees $(\mathrm{pH} 4.30)$ than in the control ( $\mathrm{pH}$ 5.20) was observed. Lees increased the survival of some L. plantarum strains, shown by a longer stationary phase, but had no effect on others.

Conclusions: The results confirm that cava lees promote the growth or survival of some lactobacilli, but as this effect is strain-dependent, case-by-case studies are required. The potential of cava lees as growth promoters deserves more attention, not only because it could reduce byproducts, but also enhance the safety of fermented foods or the effect of probiotic bacteria.

References: ${ }^{1}$ Gallardo-Chacón et al. Int. J. Food Microbiol. 2010;143:48-53; ${ }^{2}$ Shokri et al. Nat. Product Res. 2008;22:414-21.

Conflict of interest: Authors declare no conflict of interest.

Key words: cava lees / probiotic / growth promoter / starter-cultures.

Ann Nutr Metab 2018;73 (suppl 2): 1-93 DOI: $10.1159 / 000490752$ 


\section{Poster Abstract Presentations}

\section{1 \\ DEMONSTRATION OF A NEW ENZYMATIC CLEANING PROCEDURE TO REMOVE MILK FOULING IN A DAIRY PILOT PLANT}

\author{
(1)A.E. Guerrero-Navarro; (1) A.G. Rios-Castillo; ${ }^{(1)}$ C. Ripolles-Avila; \\ ${ }^{(1)}$ A.S. Hascoët; ${ }^{(1)}$ J.J. Rodríguez-Jerez; ${ }^{(2)} X$. Felipe. \\ (1)Department of Animal and Food Sciences. Universitat \\ Autònoma de Barcelona. Cerdanyola del Vallès. Spain; ${ }^{(2)} \mathrm{New}$ \\ Technologies and Food Process. IRTA. Monells. Spain.
}

Introduction: Every day the dairy industry wastes a huge amount of water, energy and time in cleaning facilities to produce safe and quality dairy products. During pasteurization or spray-drying treatments, milk is heat-treated and tends to deposit on surfaces, forming layers of organic and inorganic sediments, known as fouling. This phenomenon causes several problems because after the common alkali and acidic cleaning procedures these sediments persist on the surfaces.

Objectives: To evaluate new technology cleaning treatments to eliminate fouling under real conditions in a dairy pilot plant (IRTA, Monells, Girona, Spain) with a new enzymatic formula as an alternative to commonly used cleaning procedures (in a phase of ENTHALPY, European Union Dairy Industry project),

Methods: Batches of pasteurized and spray-dried milk were sequenced in the dairy pilot plant and the fouling produced was subsequently cleaned using a chemical or enzymatic treatment. The efficacy of the cleaning process was monitored by (i) the pressure profile during the CIP cleaning; (ii) microbiological analysis of the raw milk and the rinse water of the cleaning procedures, and (iii) the dried extract from the rinse water.

Results: A higher amount of fouling in the pilot-plant facilities was removed by the enzymatic treatment in comparison with the chemical cleaning treatment. The microbiological results were similar with both treatments and the total cost was lower for the enzymatic than the chemical cleaning process.

Conclusions: The enzymatic cleaning treatment is an alternative tool to remove fouling in dairy industries due its lower toxicity, less corrosion of the facilities and the saving of water, energy and time in comparison with the classic chemical cleaning procedures.

Conflict of interest: Authors declare no conflict of interest.

Acknowledgements: This work was supported by the European Union project FP7-KBBE-2013-7 (ENTHALPY).

Key words: enzymes / industrial cleaning / dairy fouling.

\section{2}

\section{FOOD AND WATER: A UNIVERSAL RECOGNI- TION OF THE RIGHT TO WATER AS AN ELEMENT OF HUMAN SURVIVAL.}

\author{
${ }^{(1)}$ M.F. Zaragoza-Martí; (2) A. Zaragoza-Martí \\ (1)Departamentof Legal Studies of State.. Universidad de \\ Alicante. Alicante. Spain; ${ }^{(2)}$ Nursing Departament. Universidad de \\ Alicante. Alicante. Spain.
}

Introduction: Water in sufficient quantity that is acceptable, healthy and physically accessible and affordable is vital for public health, as it is necessary for domestic use, the preparation of food or recreational purposes, as well as for safeguarding human well-being and correct food health, considering the human body is $60 \%$ water. Water is an essential nutrient without which most of the functions of our body would stop working: it allows the transport of nutrients to the cells, helps in the digestive process, contributes to the regulation of body temperature, etc.

Objectives: To carry out a bibliographic search in order to determine the relationship between the accessibility of sufficient quantity and quality of water and an adequate nutritional status.

Methods: A bibliographic review of the scientific literature was carried out with the key descriptors of food, water, sustainability and public health. Although documentation in this field is profuse, any without scientific validity or from non-binding sources was excluded.

Results: The inability to access nearby, safe and quality water resources is associated with increased water-related diseases (e.g. diarrhea-dysentery-hepatitis A-cholera-poliomyelitis) and decreased intake of basic foods (without water there is no cultivation) and health (because cleaning is not essential when water is scarce), highlighting the direct relationship between nutritional status and body water content.

Conclusions: The results show the need to claim and guarantee access to water and adequate sanitation at a universal level, as a condition for a healthy life and an adequate diet that guarantees the overcoming of water-related diseases.

Conflict of interest: Authors declare no conflict of interest.

Key words: health promotion / healthy eating / right to water / sustainable lifestyle / sanitation. 


\section{3 \\ YEAST LEES AS A FERMENTATION PROMOTER DURING BREAD PRODUCTION}

M. Riu-Aumatell; E. López-Tamames; R. Navarrete-Codina; C. Balbo; S. Hernández-Macias; M.L. Latorre-Moratalla; O. Comas-Basté; A. Díaz.

Departament de Nutrició, Ciències de l'Alimentació i Gastronomia. Campus Torribera. Universitat de Barcelona (UB). Santa Coloma de Gramenet. Spain.

Introduction: Yeast lees are defined as the residues formed at the bottom of receptacles containing wine after fermentation or during storage. Yeast lees consist mainly of inactive yeast (Saccharomyces cerevisiae), tartaric acid, inorganic and other adsorbed compounds obtained after at least 9 months of the special biological aging involved in distillation. These inactive yeast cells have a cellular wall composed of $\beta$-glucans, chitin and mannoproteins, which make lees a potentially safe promoter of sourdough fermentation, because they can be used by lactic microbiota as an added nutriment. In fact, yeast $\beta$-glucans and quitin-glucan of Aspergillus niger have been authorized as new ingredients (Decision 2011/762 CE and Decision 2011/76 CE).

Objectives: To study the importance of the fermentation process as well as the technological use of yeast lees, a by-product (200 tones each year) of sparkling wine production, as a fermentation promoter.

Methods: This study was focused on the physical and chemical characterization and the determination of the volatile profile of several sourdough and bread formulas, evaluated at various times of fermentation. For the first purpose texture profile analysis (TPA), colour analysis and $\mathrm{pH}$ were used, whereas volatile compounds were identified by Head Space Solid Phase Micro Extraction followed by Gas Chromatography coupled to Mass Spectrometry (HS-SPME-GC-MS).

Results: A large number of volatile compounds were determined throughout the fermentation process, including furans, alcohols, aldehydes, and acids. In addition, hardness, cohesiveness, and chewiness as texture parameters were observed. The addition of cava lees had a positive impact on bread fermentation.

Conclusions: This project can bring many advantages to the wine industry, such as the valorisation of a by-product, and can contribute to the improvement of sourdough fermentation time in bread-making industries.

Conflict of interest: Authors declare no conflict of interest.

Key words: lees / by-product / sourdough / volatile profile. 


\section{Nutritional on Metabolism}

\author{
Reviewers: \\ Marius Rubiralta Alcañiz \\ Universitat de Barcelona \\ Gregorio Varela Moreiras \\ Universidad CEU San Pablo
}

\section{Oral Abstract Presentations}

\section{4 \\ METABOLOMICS AS A TOOL TO STUDY MEDI- TERRANEAN CULINARY PRACTICES: COOKING AND INGREDIENTS AFFECT THE METABOLIC PROFILE OF TOMATO SOFRITO}

\author{
(1) J.F. Rinaldi de Alvarenga; ${ }^{(1,2)}$ P. Quifer-Rada; ${ }^{(1)}$ M. Illan; ${ }^{(1)} X$. Torrado- \\ Prat; ${ }^{(1,2)}$ R.M. Lamuela-Raventós. \\ ${ }^{(1)}$ Department of Nutrition, Food Sciences and Gastronomy. \\ XaRTA. INSA.UB. School of Pharmacy and Food Sciences. \\ University of Barcelona. Barcelona. Spain; ${ }^{(2)}$ CIBEROBN. Instituto \\ de Carlos III. Madrid. Spain.
}

Introduction: The cultural diversity of culinary practices, typical of each regional culture, raises the question of how food preparation and the combination of ingredients can influence the dietary patterns of a population. The Mediterranean diet is associated with health benefits and its cuisine has special characteristics that can modify the metabolic profile of its dishes and impact bioactive compound intake.

Objectives: To evaluate how home-cooking process and ingredient synergism impact the metabolomics profile of Mediterranean tomato sofrito.

Methods: Five tomato sofrito formulations, differentiated by a fortification of extra virgin olive oil (EVOO) (10\%), onion (40\%), garlic (4\%) and cooking time (60 $\mathrm{min})$, were prepared and compared with a control (5\% EVOO, $20 \%$ onion, $2 \%$ garlic and 30 $\mathrm{min})$. Freeze-dried sofritos were extracted with different dissolvents (acetonitrile, methanol and water) and analyzed by liquid chromatography coupled to high resolution mass spectrometry. Data management (feature detection, peak alignment, retention time correction, filtering) were performed using XCMS-R. T-test analysis $(\mathrm{p} \leq 0.05)$ was used to find significant features between groups. Metabolites that discriminate between groups were iden- tified using online databases with exact mass matching and confirmed by MS/MS spectra.

Results: Statistical analysis revealed possible markers that are affected by ingredients (497 features for EVOO; 1335 for onion and 490 for garlic) and cooking time (376 features). PCA analysis showed that cooking time does not influence the metabolic profile of sofrito compared to the control (R2X1X2X3 = 0.604; Q2 $\mathrm{X} 1 \mathrm{X} 2 \mathrm{X} 3=0.389$ ). PLS-DA analysis was able to differentiate between ingredient influences, onion being the one that most affected the sofrito metabolic profile.

Conclusions: A metabolomic approach proved to be a powerful tool to characterize culinary practice. Ingredients used in sofrito modify its metabolic profile, whereas a cooking time between 30 and 60 min does not affect it.

Conflict of interest: Authors declare no conflict of interest.

Key words: tomato / Mediterranean diet / cooking / phytochemicals. 


\section{Author Index}

\section{Nutrition\& Metabolism}

Anguita-Ruiz, $A$

Abete, I.

Abril-Gil, M.

Acevedo, $\mathrm{O}$

Acosta, $F$

Aguas, $Y$.

Agudelo, C

Aguilera, C

Aguirre, L.

Albaladejo, N.

Algara, $P$.

Álvarez-Sauras, M. L.

Amaro-Gahete, F.J..

Amat, $C$.

Andrés, $P$

Androutsos, $\mathrm{O}$

Antunez $C$

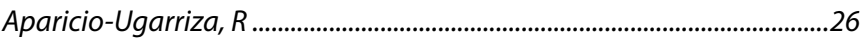

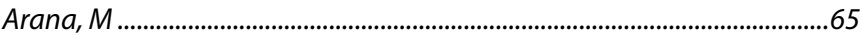

Armada, $M$....................................................................................6

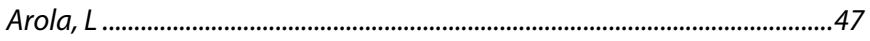

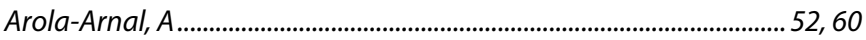

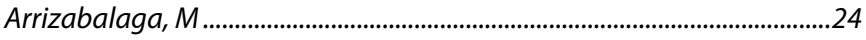

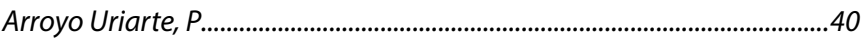

Ávila Torres, J.M............................................................................... 40, 41

Ávila, J.M................................................................................. 40, 42

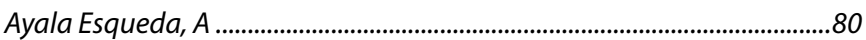

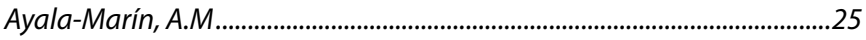

Azagra-Boronat, I............................................................................... 69, 72

Babio, N.

.. .39

Bach-Faig, A ....................................................................................... 33, 43

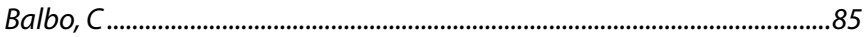

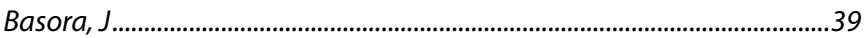

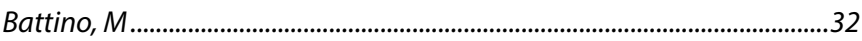

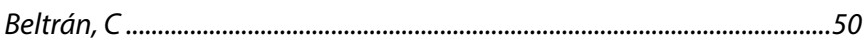

Benito-Boillos, A.............................................................................. 10, 30

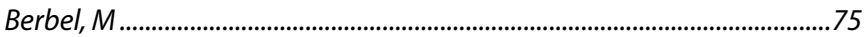

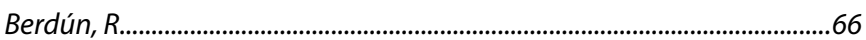

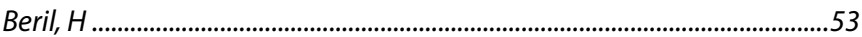

Bibiloni, M. M...............................................................................................26

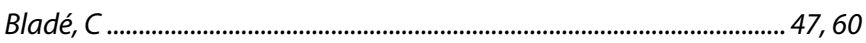

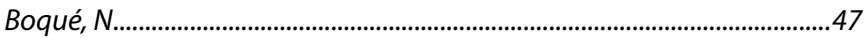

Bravo, F.I....................................................................................... 52,60

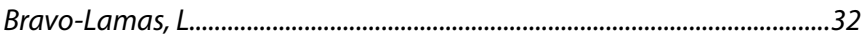

Bueno, G........................................................................................ 51, 54

Bullón Vela, V..................................................................................................4

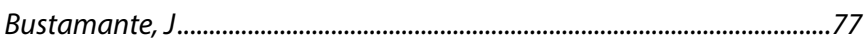

Cabañero, M. J.

Cabezas, $C$

KARGER XVII Conference of SEÑ and X Meeting of ACCA

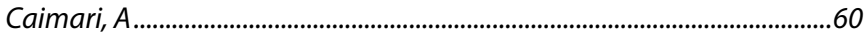

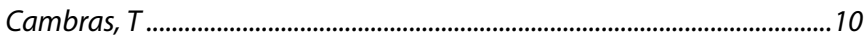

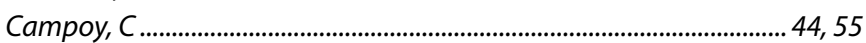

Camps-Bossacoma, M..................................................................................... 58

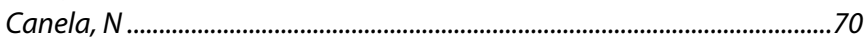

Cantero, I.................................................................................. 30,45

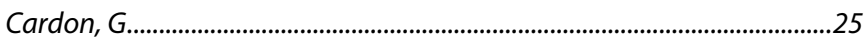

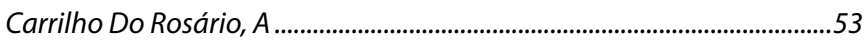

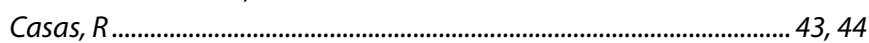

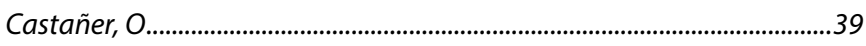

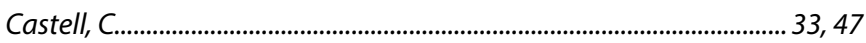

Castell, M ..................................................................................58, 69, 72

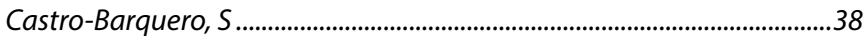

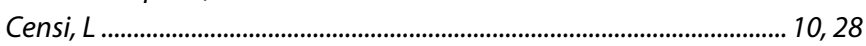

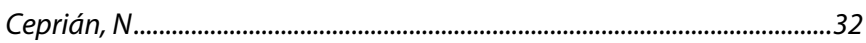

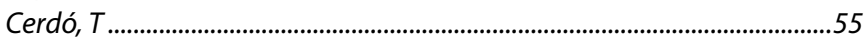

Cerrillo, I .................................................................................................6

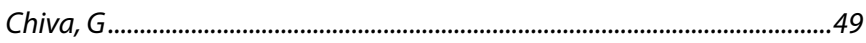

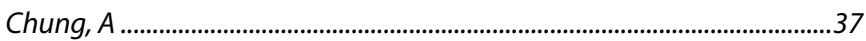

Cifuentes-Orjuela, G....................................................................................... 72

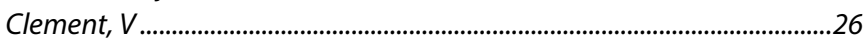

Comas-Basté, O........................................................ 10, 30, 46, 75, 83, 85

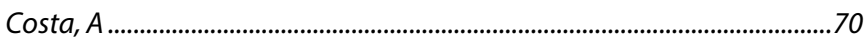

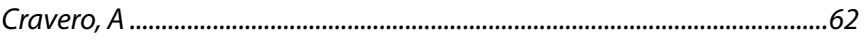

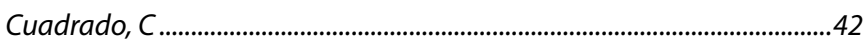

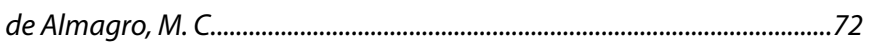

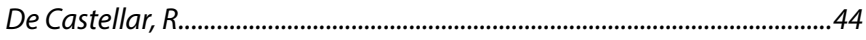

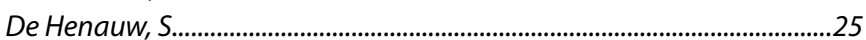

De la Fuente, M........................................................................................... 32, 71

De la Serna, M.................................................................................................. 71

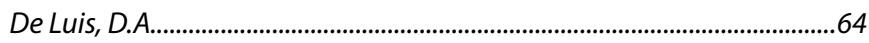

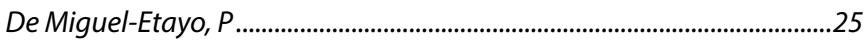

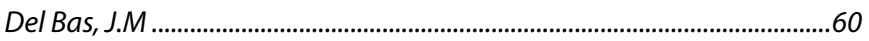

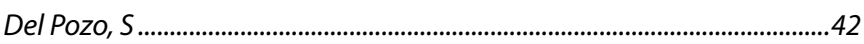

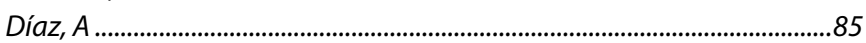

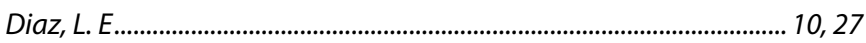

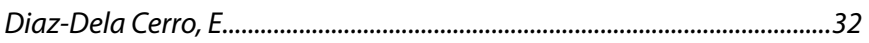

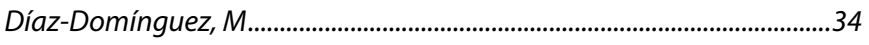

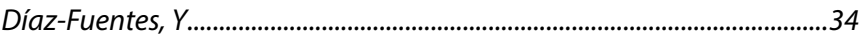

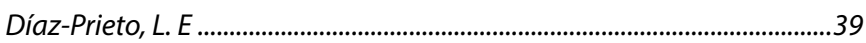

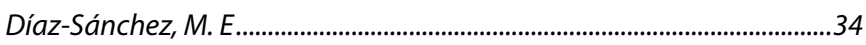

Diéguez, E..............................................................................................4

Díez-Noguera, A.............................................................................. 10, 35

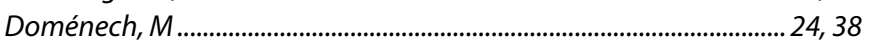

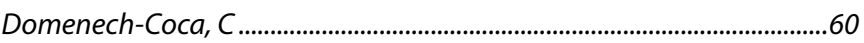

Duelo, A.................................................................................................

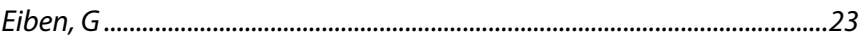

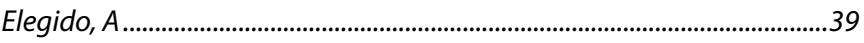

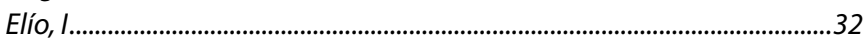

Ann Nutr Metab 2018;73 (suppl 2): 1-93 
Elorz, M. $10,30,45$

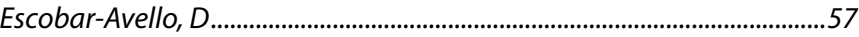

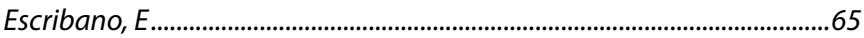

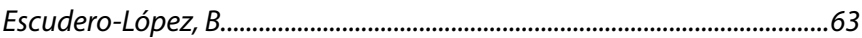

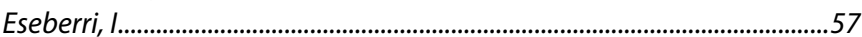

Estruch, $R$........................................................... 34, 38, 39, 43, 44, 49

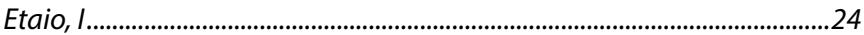

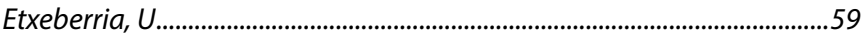

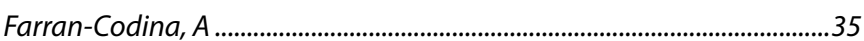

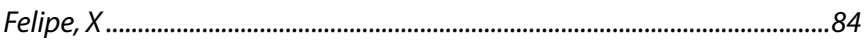

Fernández-Alvira, J.M......................................................................... 34, 38

Fernández-Pachón, M. S ..........................................................................63

Fernández-Quintela, A ...........................................................................50

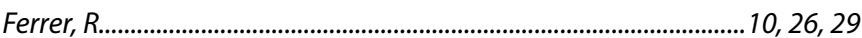

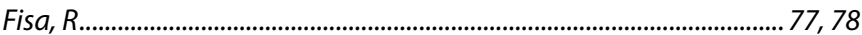

Flores-Barrantes, $P$............................................................................. 40, 42

Florez, A...................................................................................... 62,63

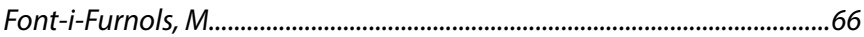

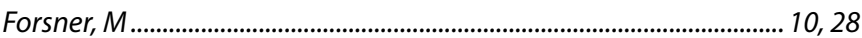

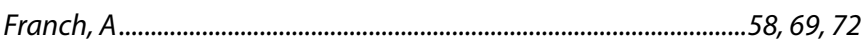

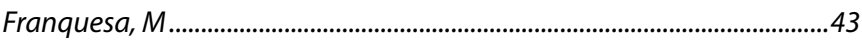

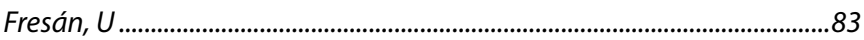

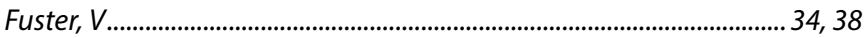

Galarregui, C................................................................................... 10, 30

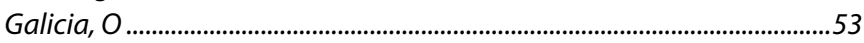

Gallego-Narbón, A ............................................................................. 36, 37

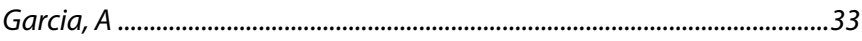

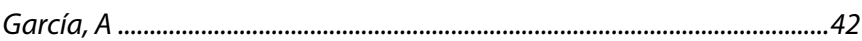

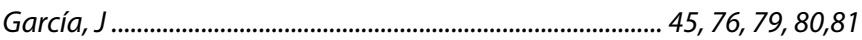

García-Fernandez, E......................................................................................4

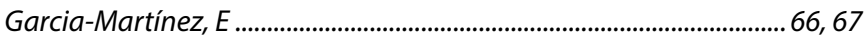

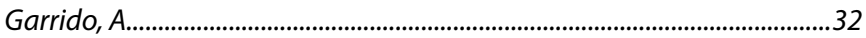

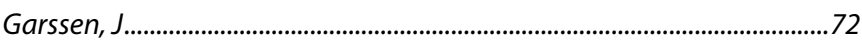

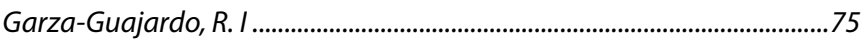

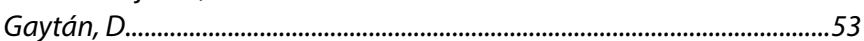

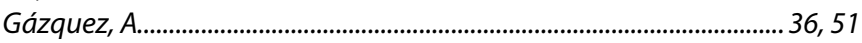

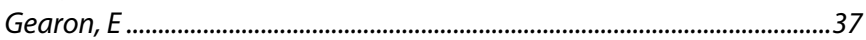

Gheorghe, $A$................................................................................ 10, 27

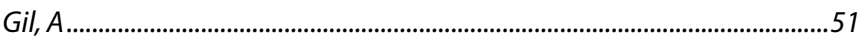

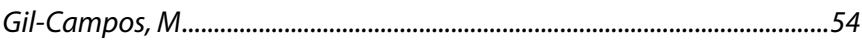

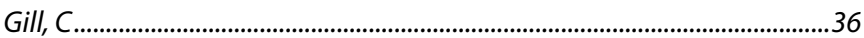

Giménez-Legarre, N .............................................................................................4 42

Goday, A.....................................................................................................

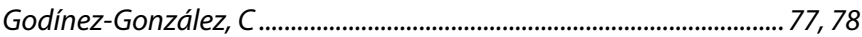

Godínez-Hernández, C ................................................................................69

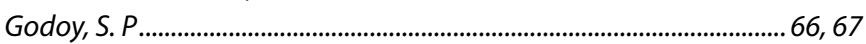

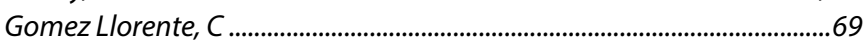

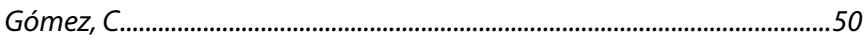

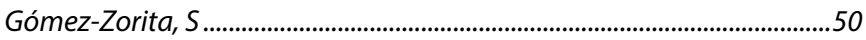

González Acevedo, 0 .....................................................................................8

González, O.................................................................................... 53, 80

González-Gil, E.M..................................................................................... 28, 54

González-Gross, M .....................................................................10, 26, 28

González-Manzano, S ................................................................................ 57

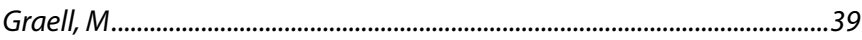

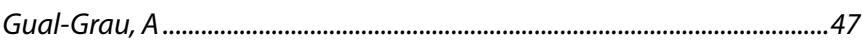

Guardiola, F.

69,77

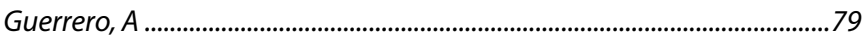

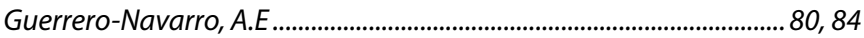

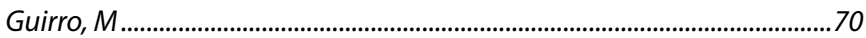

Guzmán-Mercado, E................................................................................................

Haro, $D$................................................................................. 49, 53

Hascoët, A.S ....................................................................... 80,84

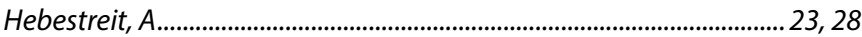

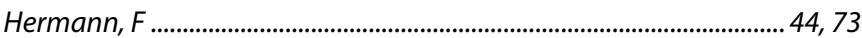

Hernández Barrera, L ........................................................................................ 80

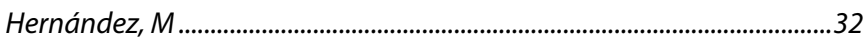

Hernández-Hernández, H..........................................................................34

Hernández-Macias, S.....................................................10, 30, 46, 83, 85

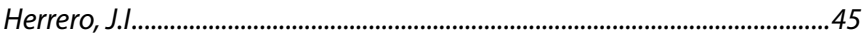

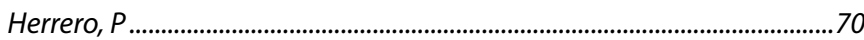

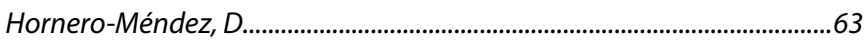

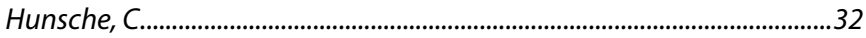

Hurtado, J.A .......................................................................... 10, 29

Huybrechts, I ....................................................................................... 10, 28

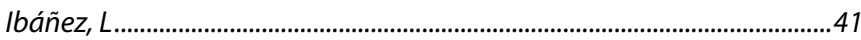

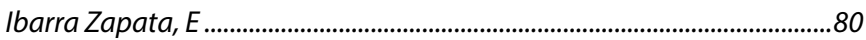

Iglesia Altaba, I................................................................................... 40, 42

Iglesia, I ....................................................................................... 41, 42

Iglesias-Carrés, L ......................................................................... 52, 60

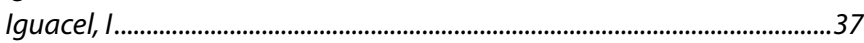

Iguacel-Azorín, I..............................................................................................25

Illan, M......................................................................................................

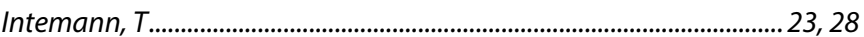

Izquierdo-Pulido, M................................................................................... 10, 35

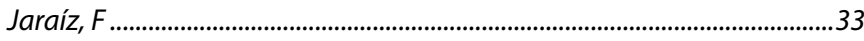

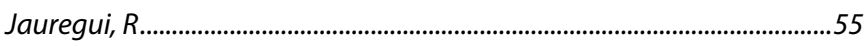

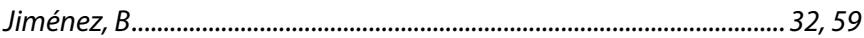

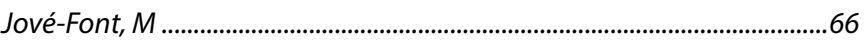

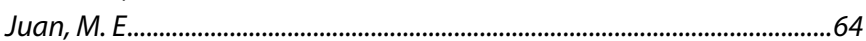

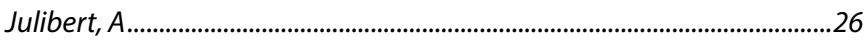

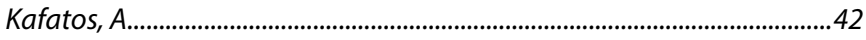

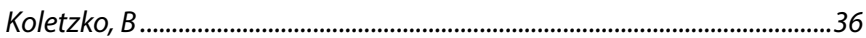

Laguna, A …………………………………………………………… 10, 29

Lamuela-Raventós, R. M .............................................34, 38, 49, 57, 59, 87

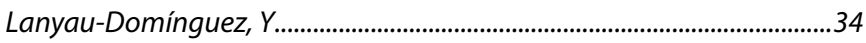

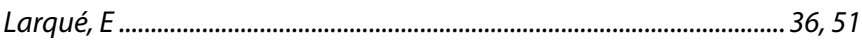

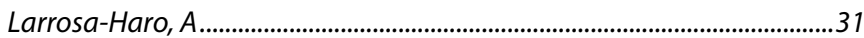

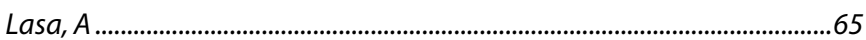

Latorre-Moratalla, M. L ............................................... 10, 30, 46, 75, 83, 85

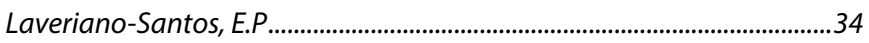

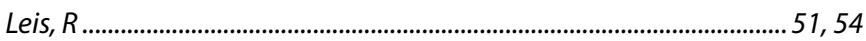

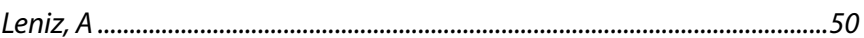

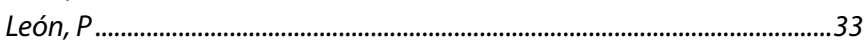

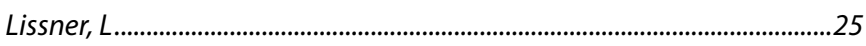

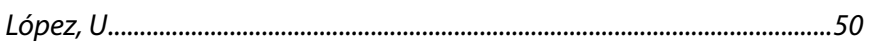

López-Santana, A.L ........................................................................ 10, 29

López-Tamames, E......................................................................... 83, 85

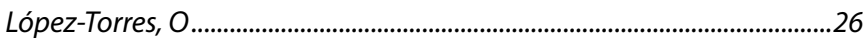

XVII Conference of SEÑ and X Meeting of ACCA 
López-Uriarte, P.J

10,29

López-Yerena, $A$.

Lozano-Castellón, J

Ludwig, I.

Macarulla, M. T. .64

Macià, $A$ .56

Macías, $L$...

$76,79,81$

Manera, $M$

Manios, $Y$.

25,42

Mantecon-Laviguerie, $H$.

.....75

Marcos, A.

$10,27,39$

Marfil, $R$.

80,81

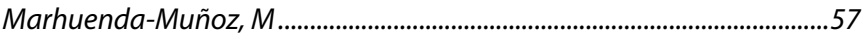

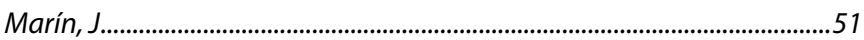

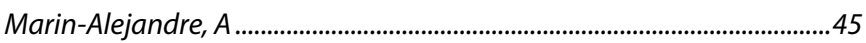

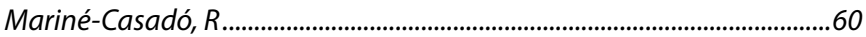

Marín-Muñoz, J............................................................................................

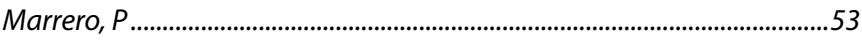

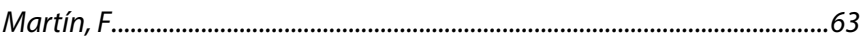

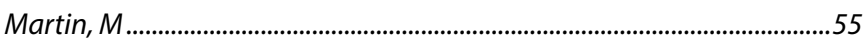

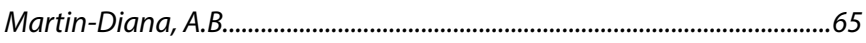

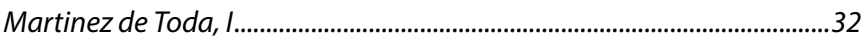

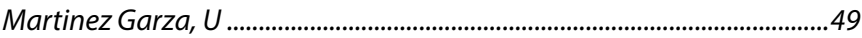

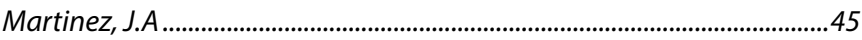

Martínez, J.A .........................................................................10, 30, 59

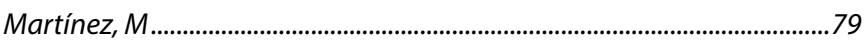

Martínez, O.................................................................................................24

Martinez-Avila, W. D ........................................................................ 10, 29

Martinez-Tellez, B....................................................................10, 27, 29

Martínez-Villaluenga, $C$...................................................................... 64, 65

Mas-Capdevila, A............................................................................... 52, 60

Massot-Cladera, M......................................................................58, 69, 72

Mauleón, J.R.........................................................................................24

Merchan-Ramirez, E.................................................................... 10, 27

Miguel Berges, M. L....................................................................40, 41, 42

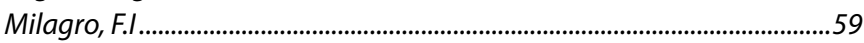

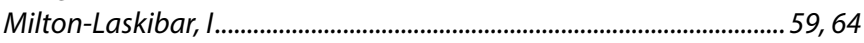

Miranda, J...........................................................................................6

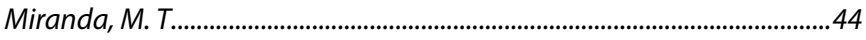

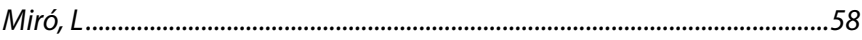

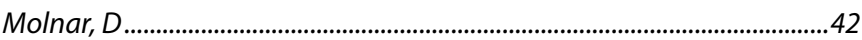

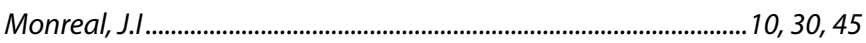

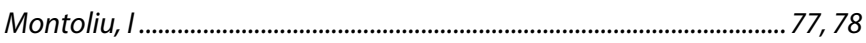

Montserrat, M .................................................................................... 32

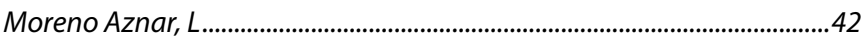

Moreno Muñoz, J.A ................................................................................72

Moreno, L......................................................................... 37, 40, 42, 51

Moreno, L. A ..............................................................10, 25, 28, 42, 54

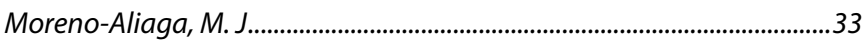

Moreno-González, R ...................................................................................64

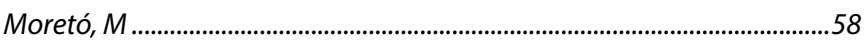

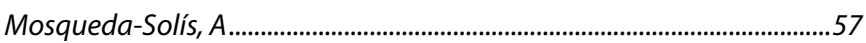

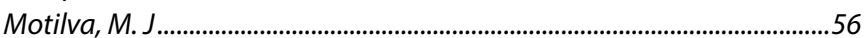

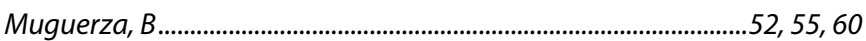

Muñoz-Esparza, N. C............................................................. 10, 30, 31, 46

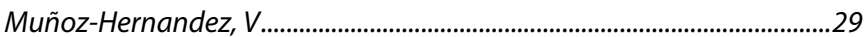

XVII Conference of SEÑ and X Meeting of ACCA

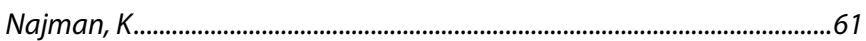

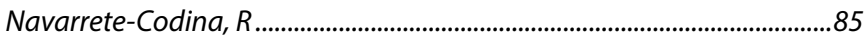

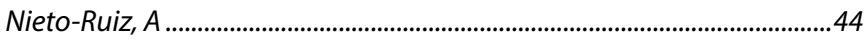

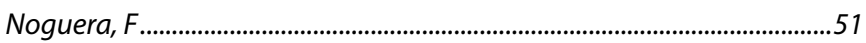

Noguera-Perea, $F$.................................................................................................. 54

Nova, $E$.............................................................................10, 27, 39

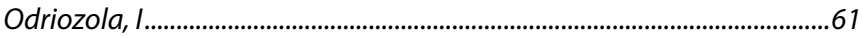

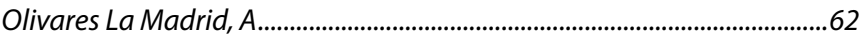

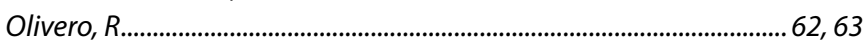

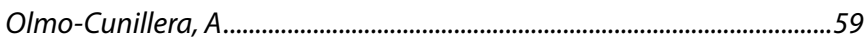

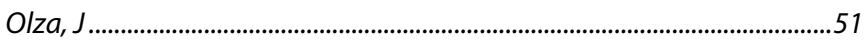

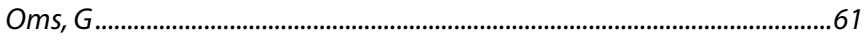

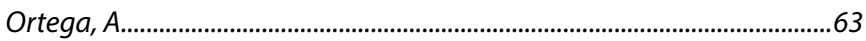

Ortiz-Alvarez, L........................................................................ 10, 27

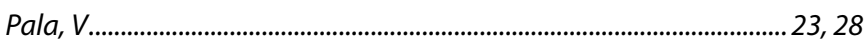

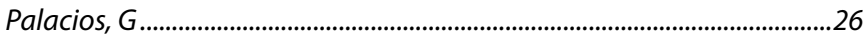

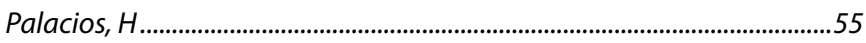

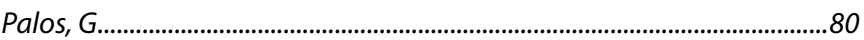

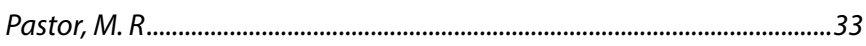

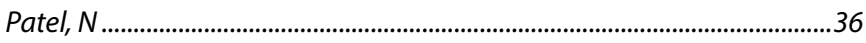

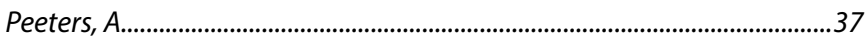

Peñas, E ...............................................................................................6

Pérez Bosch, M.................................................................................. 59, 65

Perez Felez, A.............................................................................. 10, 28

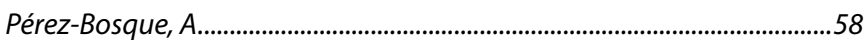

Pérez-Cano, F.J.......................................................................58, 69, 72

Petersen, $S$.................................................................................................

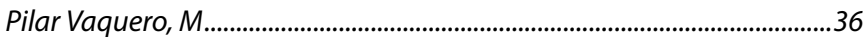

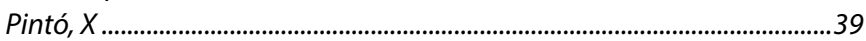

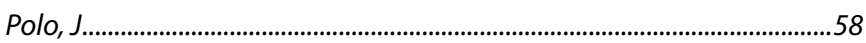

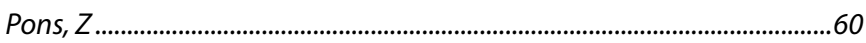

Portales-Pérez, D.P ..............................................................................69

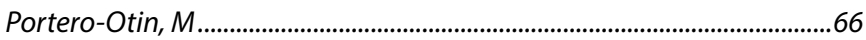

Portillo, M. P............................................................................... 54, 59

Poston, L...................................................................................................... 36

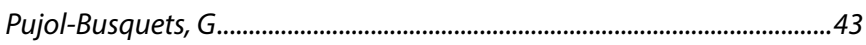

Quifer Rada, $P$.............................................................................49, 59, 87

Quintanilla-Casas, $B$................................................................................

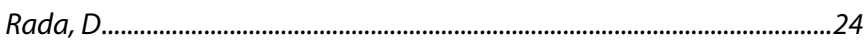

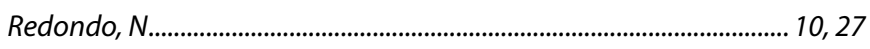

Relat, $J$..................................................................................... 49, 53

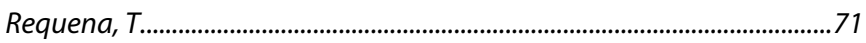

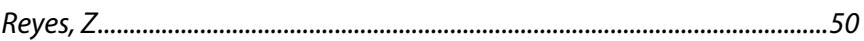

Ribó-Coll, M....................................................................................... 43, 44

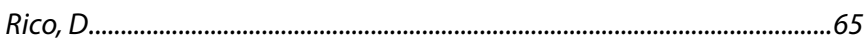

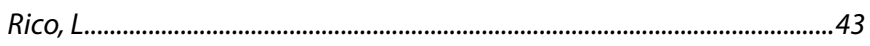

Rinaldi de Alvarenga, J.F............................................................. 57, 87

Rinaldi de Alvarenga, J.F............................................................... 57, 87

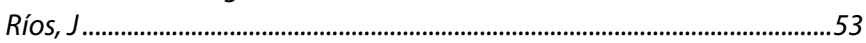

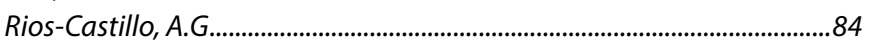

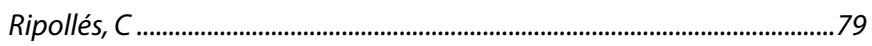

Ripolles-Avila, C............................................................................. 80,84

Riu-Aumatell, $M$............................................................................. 83,85

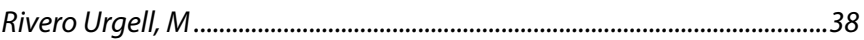

Ann Nutr Metab 2018;73 (suppl 2): 1-93 
Roca-Geronès, $X$...

77,78

Rodríguez Alonso, $P$

$. .40,41,42$

Rodríguez Martínez, $G$

Rodríguez, $A$.

Rodriguez, G.

Rodríguez, $J$.

Rodríguez, J.J..

Rodríguez, V.M.

Rodríguez-Griñolo, $R$.

Rodríguez-Jerez, J.J .

Rodríguez-Lagunas, M. J.

Rodríguez-Palmero, $C$

Rodriguez-Palmero, M...............................................................................6

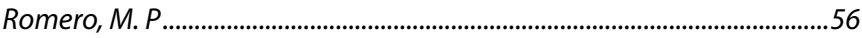

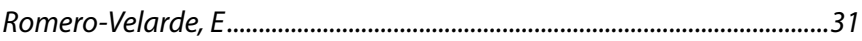

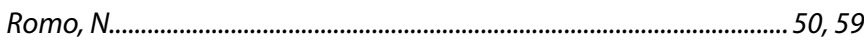

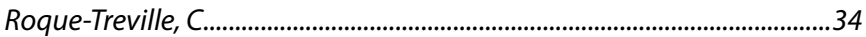

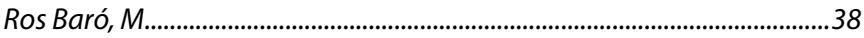

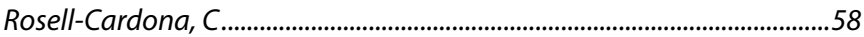

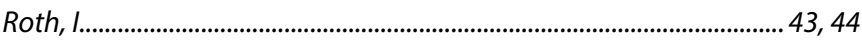

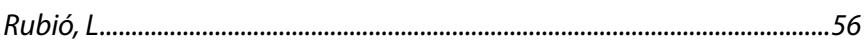

Ruiz Moreno, E........................................................................ 40, 41

Ruiz, $A$............................................................................................ 55

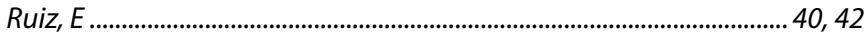

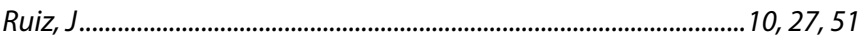

Ruiz, J.R .......................................................................... 10, 29

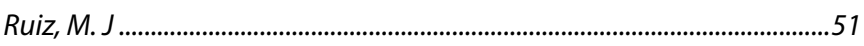

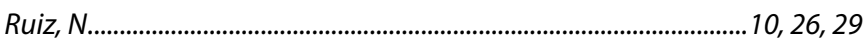

Ruiz-Alcaraz, A.J.................................................................................... 54

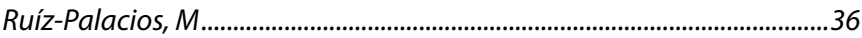

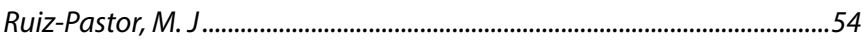

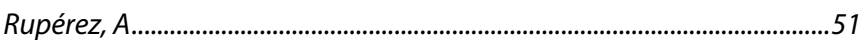

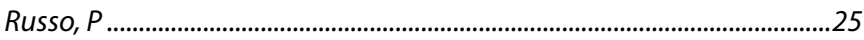

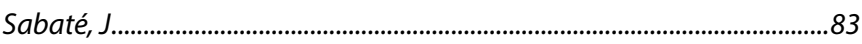

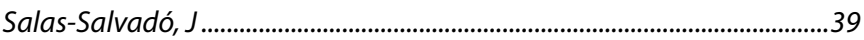

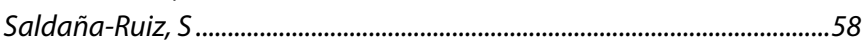

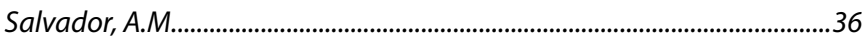

Salvador, G.................................................................................... 33, 47

Samper Villagrasa, M. P ...........................................................................4

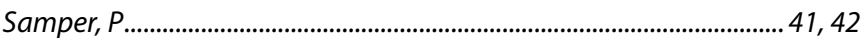

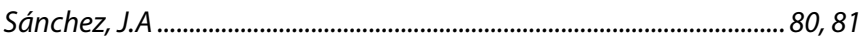

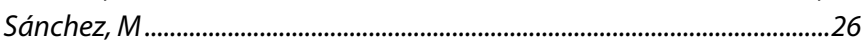

Sanchez-Delgado, G.................................................................10, 27, 29

Sánchez-Pérez, S...........................................................10, 30, 46, 75, 83

Sánchez-Rodríguez, J.A................................................................. 76, 79

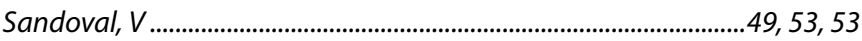

Sangrador, J...................................................................................................64

Santabárbara, J ...................................................................................25

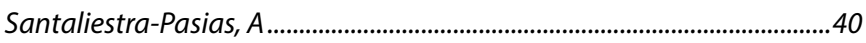

Santaliestra-Pasias, A.M .............................................................. 10, 28

Santos-Beneit, G ............................................................................. 34, 38

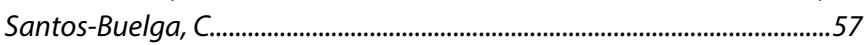

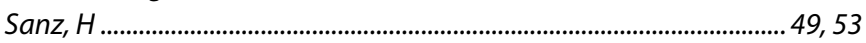

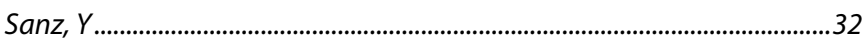

Sánzhez-Campillo, M..................................................................................... 51

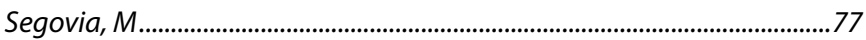

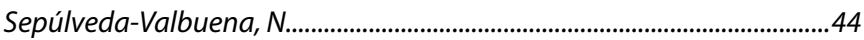

Shamirian-Pulido, L......................................................................................4

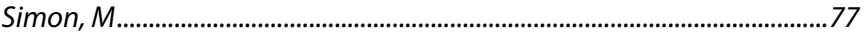

Sjostrom, $M$........................................................................... 10, 28

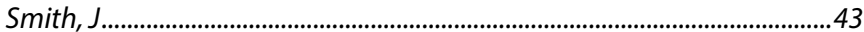

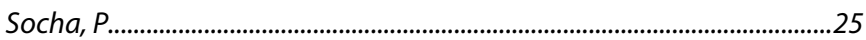

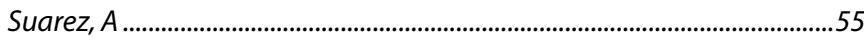

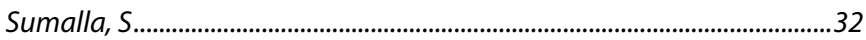

Summerbell, $C$.............................................................................................25

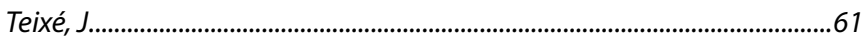

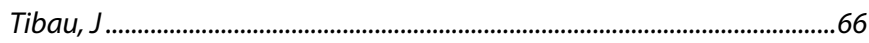

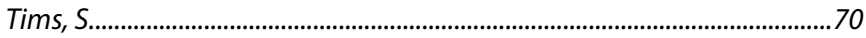

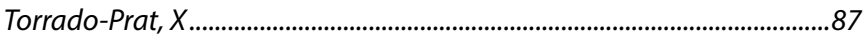

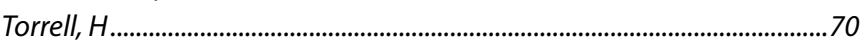

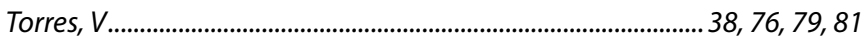

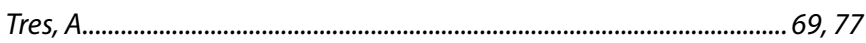

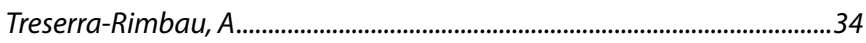

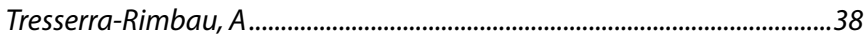

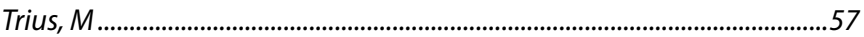

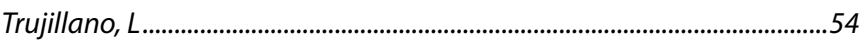

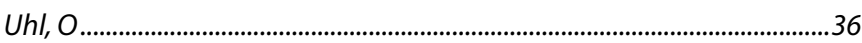

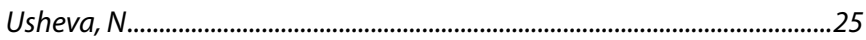

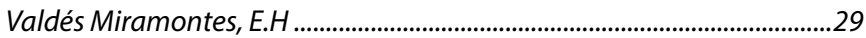

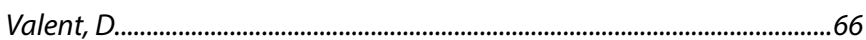

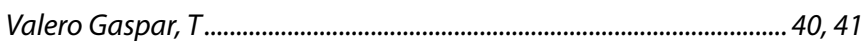

Valero, $T$................................................................................... 40, 42

Vallverdú-Queralt, $A$........................................................................... 57, 59

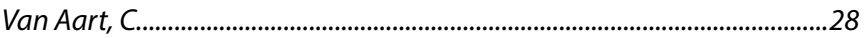

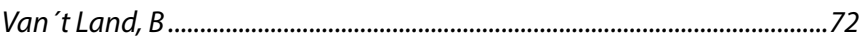

Varela Moreiras, G ..........................................................................40, 41, 42

Vargas-Morales, J.M..................................................................................6

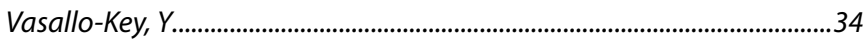

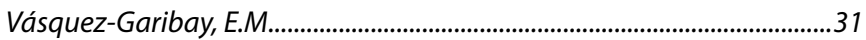

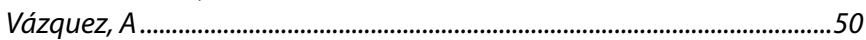

Vázquez-Maldonado, D .................................................................................69

Veciana-Nogués, M. T...................................................10, 30, 46, 75, 83

Vega-Cárdenas, M .........................................................................................69

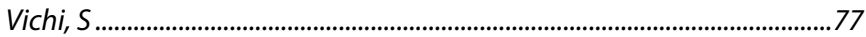

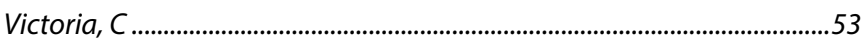

Vidal-Carou, M. C.......................................................... 10, 30, 31, 46, 75, 83

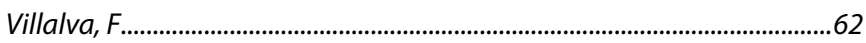

Villavisencio, $B$........................................................................... 10, 27

von Bergen, M.....................................................................................

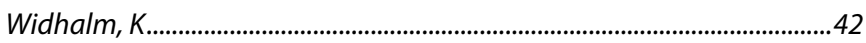

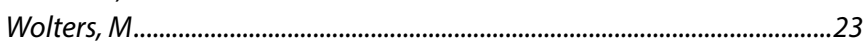

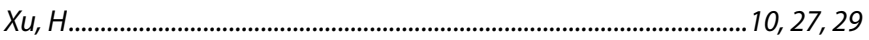

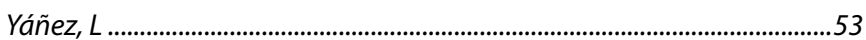

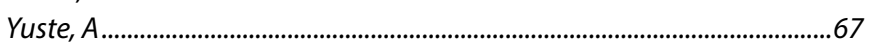

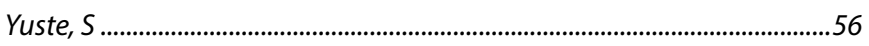

Zapatera, $B$................................................................................. 36,37

Zaragoza, $A$.........................................................................10, 26, 29

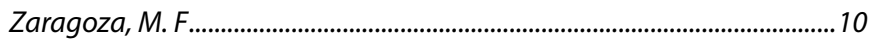

XVII Conference of SEÑ and X Meeting of ACCA 


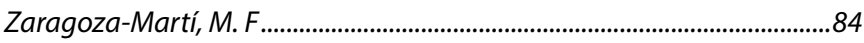

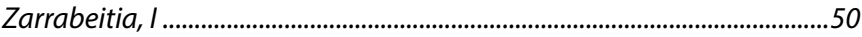

Zeron-Rugerio, M. F........................................................................ 10, 35

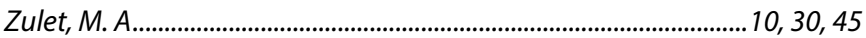

Backholer, K.................................................................................................... 37

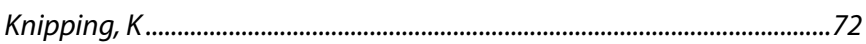


